\title{
Álgebra de semigrupo na compactificação de Stone-Čech de semigrupos discretos
}

\author{
Matheus Koveroff Bellini
}

DisSERTAÇÃO APRESENTADA

$\mathrm{AO}$

Instituto De MatemÁticA E EstatísticA

DA

UnIVERSIDADE DE SÃO PAUlo

PARA

OBTENÇÃO DO TÍTULO

$\mathrm{DE}$

Mestre em MAtemáticA

Programa: Mestrado em Matemática

Orientador: Prof. Dr. Artur Hideyuki Tomita

Durante o desenvolvimento deste trabalho o autor recebeu auxílio financeiro da FAPESP

(Processo 2015/19857-4)

São Paulo, outubro de 2017 


\section{Álgebra de semigrupo na compactificação de Stone-Čech de semigrupos discretos}

Esta versão da dissertação contém as correções e alterações sugeridas pela Comissão Julgadora durante a defesa da versão original do trabalho, realizada em 11/12/2017. Uma cópia da versão original está disponível no Instituto de Matemática e Estatística da Universidade de São Paulo.

Comissão Julgadora:

- Prof. Dr. Artur Hideyuki Tomita (orientador) - IME-USP

- Prof. Dr. Leandro Fiorini Aurichi - ICMC-USP

- Prof. Dr. Vladimir Pestov - University of Ottawa 


\section{Sumário}

1 Preliminares 1

1.1 Semigrupos, Ideais e Idempotentes . . . . . . . . . . . . . . . . . . . . . 1

1.2 Semigrupos Topológicos à Direita Compactos . . . . . . . . . . . . . . . . . . . 6

1.3 Compactificação de Stone-Čech de Espaços Discretos . . . . . . . . . . . . . . . . 7

1.4 A Compactificação de Stone-Čech de um Semigrupo Discreto . . . . . . . . . . . . 10

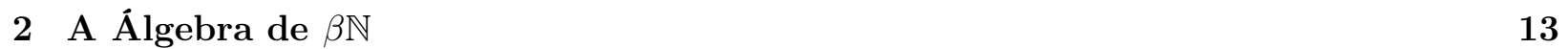

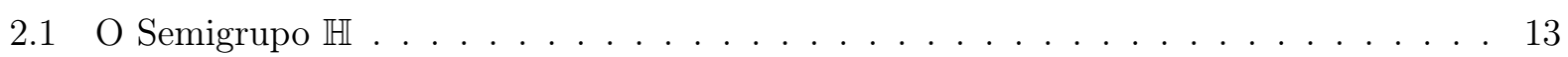

2.2 O Ideal $\mathbb{N}^{*}+\mathbb{N}^{*}$, o Fecho de $K(\beta \mathbb{N}), \mathcal{U}_{\kappa}(S)$, Somas e Produtos $\ldots \ldots \ldots \ldots$

$2.3 \mathbb{N}^{*}$ não contém uma cópia algébrica e topológica de $\beta \mathbb{N} \ldots \ldots \ldots$. . . . . . . . . . . . 29

2.4 Subgrupos discretos em $\beta \mathbb{N} \ldots \ldots \ldots \ldots \ldots$

2.5 Cadeias de idempotentes . . . . . . . . . . . . . . . . . . . 45

3 A Álgebra de $\beta S \quad 55$

3.1 Sobre a Raridade de Produtos em $S^{*} \ldots \ldots \ldots \ldots \ldots \ldots$

3.2 Cancelabilidade à direita em $\beta S \ldots \ldots \ldots \ldots \ldots \ldots \ldots$

3.3 Cópias de $\mathbb{H}$ em $S^{*} \ldots \ldots \ldots \ldots \ldots \ldots \ldots \ldots \ldots \ldots \ldots$

3.4 O Teorema de Zelenyuk . . . . . . . . . . . . . . . . . . 85

$\begin{array}{ll}\text { A Grupos Abelianos Divisíveis } & 101\end{array}$ 


\section{Capítulo 1}

\section{Preliminares}

Denotaremos por $\omega=\{0,1,2, \ldots\}$ o primeiro ordinal e cardinal infinito e por $\mathbb{N}$ o conjunto $\{1,2,3, \ldots\}$, ou seja, $\omega \backslash\{0\}$. Como ordinal e cardinal, um número natural $n \in \mathbb{N}$ é o conjunto $\{0,1, \ldots, n-1\}$. Denota-se também $\mathfrak{c}=2^{\omega}=|\mathcal{P}(\mathbb{N})|$.

Dada função $f$ e dado $A$ contido no domínio de $f, f[A] \doteq\{f(x): x \in A\}$. Dado qualquer conjunto $B, f^{-1}[B] \doteq\{x \in \operatorname{dom} f: f(x) \in B\}$. Dizemos que a função é finita-por-unitários se para todo $x, f^{-1}[\{x\}]$ for finito.

Dado um conjunto $A, \mathcal{P}_{f}(A) \doteq\{F: \varnothing \neq F \subset A$ e $F$ é finito $\}$.

Como esta dissertação é sobre a álgebra de semigrupo de $\beta S$ para $S$ semigrupo discreto - em particular $(\beta \mathbb{N},+)$ e $(\beta \mathbb{N}, \cdot)$-, nesta seção apresentaremos as definições necessárias e os teoremas mais relevantes para fundamentar o assunto. Mais detalhes podem ser encontrados nos capítulos 1 , 2,3 e 4 de [1].

\subsection{Semigrupos, Ideais e Idempotentes}

Definição 1.1. Um semigrupo é um par $(S, *)$ em que $S$ é um conjunto não-vazio e * é uma operação binária associativa sobre $S$.

Definição 1.2. Dado $A \neq \varnothing$, o semigrupo livre sobre $A$ é o conjunto de todas as sequências finitas de elementos de $A$, com a operação definida pela concatenação de sequências.

Definição 1.3. Sejam $(S, \cdot)$ um semigrupo, $O$ um conjunto linearmente ordenado por $<$, e $\left(x_{o}\right)_{o \in O}$ uma $O$-sequência em $S$ (ou seja, uma função de $O$ em $S$ ). Definimos, para cada $F \in \mathcal{P}_{f}(O)$, $\prod_{o \in F} x_{o}$ como o produto na ordem crescente de índices.

Dizemos que $\left(x_{o}\right)_{o \in O}$ satisfaz a unicidade dos produtos finitos, ou que possui produtos finitos distintos, se sempre que $F, G \in \mathcal{P}_{f}(O)$ e $\prod_{o \in F} x_{o}=\prod_{o \in G} x_{o}$, vale que $F=G$.

Além disso, define-se $F P\left(\left(x_{o}\right)_{o \in O}\right)=\left\{\prod_{o \in F} x_{o}: F \in \mathcal{P}_{f}(O)\right\}$. 
Definição 1.4. Sejam $(S, *)$ e $(T, \cdot)$ semigrupos.

(a) Um homomorfismo de $S$ em $T$ é uma função $\varphi: S \rightarrow T$ tal que $\varphi(x * y)=\varphi(x) \cdot \varphi(y)$, para todos $x, y \in S$.

(b) Um isomorfismo de $S$ em $T$ é um homomorfismo de $S$ em $T$ que é bijetor.

(c) $S$ e $T$ são isomorfos se existir um isomorfismo de $S$ em $T$. Neste caso denotamos $S \cong T$.

Por conveniência, passaremos a denotar $x * y$ por $x y$ e diremos "seja $S$ um semigrupo" sem especificar a operação, caso não surjam ambiguidades.

Definição 1.5. Sejam $S$ um semigrupo e $a \in S$.

(a) a é uma identidade à esquerda de $S$ se, para todo $x \in S$, $a x=x$.

(b) a é uma identidade à direita de $S$ se, para todo $x \in S, x a=x$.

(c) $a$ é uma identidade de $S$ se $a$ for uma identidade à direita e uma identidade à esquerda de $S$.

Definição 1.6. Seja $S$ um semigrupo.

(a) $S$ é comutativo se, para todos $x, y \in S, x y=y x$.

(b) O centro de $S$ é $Z(S)=\{x \in S$ : para todo $y \in S, x y=y x\}$.

(c) Dado $x \in S, \lambda_{x}: S \rightarrow S$ é definido por $\lambda_{x}(y)=x y$.

(d) Dado $x \in S, \rho_{x}: S \rightarrow S$ é definido por $\rho_{x}(y)=y x$.

Definição 1.7. Seja $S$ um semigrupo.

(a) $x \in S$ é cancelável à direita se dados $y, z \in S$ tais que $y x=z x$, tem-se que $y=z$.

(b) $x \in S$ é cancelável à esquerda se dados $y, z \in S$ tais que $x y=x z$, tem-se que $y=z$.

(c) $S$ é cancelativo à direita se todo $x \in S$ é cancelável à direita.

(d) $S$ é cancelativo à esquerda se todo $x \in S$ é cancelável à esquerda.

(e) $S$ é cancelativo se for cancelativo à esquerda e à direita.

Definição 1.8. Seja $S$ um semigrupo.

(a) $x \in S$ é um idempotente se $x x=x$.

(b) $E(S)=\{x \in S: x x=x\}$.

(c) $T$ é um subsemigrupo de $S$ se $T \subset S$ e $T$ é um semigrupo com a restrição da operação de $S$. 
(d) $T$ é um subgrupo de $S$ se $T \subset S$ e $T$ é um grupo com a restrição da operação de $S$.

Definição 1.9. Seja $S$ um semigrupo.

(a) $L$ é um ideal à esquerda de $S$ se $\varnothing \neq L \subset S$ e $S L \subset L$.

(b) $R$ é um ideal à direita de $S$ se $\varnothing \neq L \subset S$ e $R S \subset R$.

(c) $I$ é um ideal de $S$ se $I$ for um ideal à esquerda e um ideal à direita de $S$.

Definição 1.10. Seja $S$ um semigrupo.

(a) $L$ é um ideal à esquerda minimal se $L$ for um ideal à esquerda de $S$ tal que dado $J$ ideal à esquerda tal que $J \subset L$, tem-se que $J=L$.

(b) $R$ é um ideal à direita minimal se $R$ for um ideal à direita de $S$ tal que dado $J$ ideal à direita tal que $J \subset R$, tem-se que $J=R$.

(c) $S$ é simples à esquerda se $S$ for o único ideal à esquerda de $S$.

(d) $S$ é simples à direita se $S$ for o único ideal à direita de $S$.

(e) $S$ é simples se $S$ for o único ideal de $S$.

Definição 1.11. Sejam $S$ um semigrupo e $e, f \in E(S)$.

(a) $e \leq_{L} f$ se e só se $e=e f$.

(b) $e \leq_{R} f$ se e só se $e=f e$.

(c) $e \leq f$ se e só se $e=f e=e f$.

Vale que $\leq_{L}, \leq_{R}$ e $\leq$ são reflexivas e transitivas, e $\leq$ é antissimétrica. Além disso, tem-se que são equivalentes:

1. e é minimal com relação a $\leq_{L}$;

2. e é minimal com relação a $\leq_{R}$;

3. e é minimal com relação a $\leq$.

De forma que é válido definir:

Definição 1.12. Seja $S$ um semigrupo. $e \in E(S)$ é um idempotente minimal se $e$ for minimal com relação a qualquer uma das pré-ordens $\leq_{L}, \leq_{R}$ ou $\leq$.

Lembramos que um elemento $x$ de uma pré-ordem $P$ é minimal se sempre que $y P x$, então vale que $x P y$ (e não necessariamente $x=y$ ). 
Definição 1.13. Seja $S$ um semigrupo. Se $S$ possui um menor ideal, denotamo-lo por $K(S)$.

Teorema 1.14. Seja $S$ um semigrupo. Se $S$ possui um ideal à esquerda minimal, então $K(S)$ existe e $K(S)=\bigcup\{L: L$ é um ideal à esquerda minimal de $S\}$.

Teorema 1.15. Suponha que $S$ contém um ideal à esquerda minimal que possui um idempotente. Então:

(a) Todo ideal à esquerda de $S$ contém um ideal à esquerda minimal.

(b) Todo ideal à esquerda minimal possui um idempotente.

(c) Existe um ideal à direita minimal que possui um idempotente. (Logo todo ideal à direita contém um ideal à direita minimal que possui um idempotente.)

(d) Dado $T \subset S, T$ é um ideal à esquerda minimal se e somente se existe $e \in E(K(S))$ tal que $T=S e$.

Teorema 1.16. Seja $S$ um semigrupo, suponha que existe um ideal à esquerda minimal que possui um idempotente e seja e $\in E(S)$. São equivalentes:

(a) Se é um ideal à esquerda minimal.

(b) Se é simples à esquerda.

(c) eSe é um grupo.

(d) eSe é o maior grupo que tem e como identidade.

(e) eS é um ideal à direita minimal.

(f) eS é simples à direita.

(g) e é um idempotente minimal.

(h) $e \in K(S)$.

(i) $K(S)=S e S$.

Teorema 1.17. Suponha que $S$ contém um ideal à esquerda minimal que possui um idempotente. Então:

(a) Dado e idempotente, existe $f$ idempotente tal que $f \leq e$.

(b) Dados $L$ ideal à esquerda minimal e $R$ ideal à direita minimal, existe um idempotente e tal que $e \in R \cap L=R L=e S e$ e eSe é grupo. 
(c) Os ideais à esquerda minimais de $S$ particionam $K(S)$; os ideais à direita minimais de $S$ particionam $K(S)$; os grupos maximais de $K(S)$ particionam $K(S)$.

(d) Suponha T subsemigrupo de $S$ que também contém um ideal à esquerda que possui um idempotente. Se $K(S) \cap T \neq \varnothing$, então $K(T)=K(S) \cap T$.

(e) São equivalentes:

(i) $s \in K(S)$.

(ii) Para todo $t \in S, s \in$ Sts.

(iii) Para todo $t \in S, s \in$ stS.

(iv) Para todo $t \in S, s \in S t s \cap s t S$. 


\subsection{Semigrupos Topológicos à Direita Compactos}

Definição 1.18. Um semigrupo topológico à direita é uma tripla $(S, *, \tau)$ em que $(S, *)$ é um semigrupo, $(S, \tau)$ é um espaço topológico e, para todo $x \in S, \rho_{x}: S \rightarrow S$ é contínua.

Costumeiramente, dir-se-á "seja $S$ um semigrupo topológico à direita" quando disso não resultarem ambiguidades.

Definição 1.19. Seja $S$ um semigrupo topológico à direita. O centro topológico de $S$ é $\Lambda(S)=\left\{x \in S: \lambda_{x}\right.$ é contínua $\}$.

Teorema 1.20. Seja $S$ um semigrupo topológico à direita compacto. Então $E(S) \neq \varnothing$.

Corolário 1.21. Seja $S$ um semigrupo topológico à direita compacto. Então todo ideal à esquerda contém um ideal à esquerda minimal, ideais à esquerda minimais são fechados, e todo ideal à esquerda minimal possui um idempotente.

Dessa forma, se $S$ é um semigrupo topológico à direita compacto, valem todos os resultados listados em 1.14, 1.15, 1.16 e 1.17.

Teorema 1.22. Sejam $S$ um semigrupo topológico à direita e $R$ um ideal à direita de $S$. Então cl $R$ é um ideal à direita de $S$.

A seguir, um teorema cuja demonstração do item (a) será dada para exemplificar a interação entre as estruturas algébrica e topológica de $S$.

Teorema 1.23. Seja $S$ um semigrupo topológico à direita compacto, e suponha que $Z(S)$ é denso em $S$.

(a) Seja L um ideal à esquerda de $S$. Então cl L é um ideal à esquerda de $S$.

(b) Seja $R$ um ideal à direita de $S$. Então cl $R$ é um ideal de $S$.

(c) Seja e $\in E(K(S))$. Então $\operatorname{cl}(e S e)=S e$.

Demonstração. (a): Sejam $x \in \operatorname{cl} L$ e $y \in S$. Seja $U$ vizinhança de $y x . y x=\rho_{x}(y)$, logo seja $V$ vizinhança de $y$ tal que $\rho[V] \subset U$. Seja $z \in Z(S) \cap V$. Então $\rho_{x}(z)=z x=x z=\rho_{z}(x) \in U$, logo seja $W$ vizinhança de $x$ tal que $\rho_{z}[W] \subset U$. Seja $w \in W \cap L$. Então $\rho_{z}(w)=w z=z w \in U \cap L$. Logo $y x \in \operatorname{cl} L$. 


\subsection{Compactificação de Stone-Čech de Espaços Discretos}

Esta seção tem o propósito de fixar notações, já que o assunto é amplamente conhecido, e assim não é necessária uma longa exposição de definições e teoremas.

Definição 1.24. Dado $D$ espaço topológico discreto, $\beta D=\{p: p$ é um ultrafiltro sobre $D\}$, como conjunto. Definindo, para cada $A \subset D, \widehat{A}=\{p \in \beta D: A \in p\}$, temos que $\{\widehat{A}: A \subset D\}$ é uma base para uma topologia em $\beta D$. $\beta D$ com esta topologia é uma compactificação de Stone-Čech de $D$; ao identificar cada $x \in D$ com seu ultrafiltro principal, podemos considerar $D \subset \beta D$.

Assim, temos que:

(i) Os clopens são exatamente os conjuntos da forma $\widehat{A}$, para $A \subset D$. (Portanto $\beta D$ é zerodimensional.)

(ii) Para todo $A \subset D, \widehat{A}=\operatorname{cl}_{\beta D} A$.

(iii) Os pontos isolados de $\beta D$ são exatamente os pontos de $D$.

(iv) Dado $U \subset \beta D$ aberto, $\operatorname{cl}_{\beta D} U$ também é aberto. (Ou seja, $\beta D$ é extremamente desconexo.)

Definição 1.25. (a) Seja $A \subset D$. Então $A^{*}=\widehat{A} \backslash A$.

(b) Dado $T \subset D$, podemos identificar $p \in \widehat{T} \operatorname{com}\{A \cap T: A \in p\} \in \beta T$, e assim diremos que $\beta T \subset \beta D$.

(c) Sejam $Y$ compacto e $f: D \rightarrow Y$, denotaremos por $\widetilde{f}$ a extensão contínua de $f$ a $\beta D$.

(d) Seja $\kappa$ cardinal. Então $\mathcal{U}_{\kappa}(D)=\{p \in \beta D: \forall A \in p,|A| \geq \kappa\}$.

(e) Seja $\kappa$ cardinal infinito, e seja $\mathcal{A}$ uma coleção de conjuntos. Então $\mathcal{A}$ possui a propriedade da interseção finita $\kappa$-uniforme (também chamada de $\kappa$-p.i.f.) se dado qualquer $\mathcal{F} \in \mathcal{P}_{f}(\mathcal{A})$, $|\cap \mathcal{F}| \geq \kappa$.

(f) Dado $\mathcal{A}$ filtro em $D, \widehat{\mathcal{A}}=\{p \in \beta D: \mathcal{A} \subset p\}$.

Com estas definições, valem os seguintes resultados:

Teorema 1.26. (a) Suponha que $f: D \rightarrow D$ não possui pontos fixos. Então $D$ pode ser particionado em três células $A_{1}, A_{2}, A_{3}$ tais que para cada $i, A_{i} \cap f\left[A_{i}\right]=\varnothing$. Como corolário, $\tilde{f}: \beta D \rightarrow \beta D$ também não possui pontos fixos.

(b) Se $D$ é infinito, então todo $G_{\delta}$ de $D^{*}$ possui interior não-vazio em $D^{*}$. Como consequência, toda união enumerável de nunca-densos de $D^{*}$ é nunca-densa em $D^{*}$. 
(c) Sejam $A$ e $B$ subconjuntos $\sigma$-compactos de $\beta D$ ou de $D^{*}$. Então $(\operatorname{cl} A) \cap(\operatorname{cl} B) \subset(A \cap(\operatorname{cl} B)) \cup$ $((\operatorname{cl} A) \cap B)$.

(d) Seja $D$ infinito de cardinalidade $\kappa$, e seja $\mathcal{A} \subset \mathcal{P}(D)$ tal que $|\mathcal{A}| \leq \kappa$ e $\mathcal{A}$ possui a $\kappa$-p.i.f. Então $\left|\left\{p \in \mathcal{U}_{\kappa}(D): \mathcal{A} \subset p\right\}\right|=2^{2^{\kappa}}$. Em particular, $\left|\mathcal{U}_{\kappa}(D)\right|=|\beta D|=2^{2^{\kappa}}$.

(e) Sejam $D$ e E espaços discretos e $f: D \rightarrow E \subset \beta E$. Então para cada $p \in \beta D, \widetilde{f}(p)=\{A \in$ $\left.E: f^{-1}[A] \in p\right\}$. Logo, se $f$ é injetora, $\tilde{f}$ é injetora; e se $f$ é sobrejetora, $\tilde{f}$ é sobrejetora. E se $B \in p$, então $f[B] \in \widetilde{f}(p)$.

(f) Dado $\mathcal{A}$ filtro em $D, \widehat{\mathcal{A}}$ é um fechado de $\beta D$.

(g) Dado $A \subset \beta D, \bigcap A$ é um filtro em $D$ e $\operatorname{cl}_{\beta D} A=\widehat{\bigcap A}$.

(h) Sejam $X$ espaço topológico, $f: \beta D \rightarrow X$ contínua, e $p \in \beta D$. Então dada $U$ vizinhança de $f(p), D \cap f^{-1}[U] \in p$.

(i) Seja $X$ espaço topológico zero-dimensional e seja $Y \subset X$ compacto. Então os clopens de $Y$ são da forma $C \cap Y$ em que $C$ é clopen em $X$. Em particular: se $D$ é infinito, então todo clopen não-vazio de $D^{*}$ é da forma $A^{*}$ para $A \subset D$ infinito.

(j) Seja $D$ infinito e seja $A \subset \beta D$ infinito e fechado. Então A contém uma cópia topológica de $\beta \mathbb{N}$. Em particular, $|A| \geq 2^{\mathfrak{c}}$.

Agora, definiremos limites e p-limites e listaremos suas propriedades básicas, que são similares com as propriedades de redes.

Definição 1.27. (a) Sejam $X$ e $Y$ espaços topológicos, $A \subset X, f: A \rightarrow Y, x \in \operatorname{cl}_{X} A$ e $y \in Y$. Dizemos que $\lim _{a \rightarrow x} f(a)=y$ se para toda vizinhança $U$ de $y$ existir $V$ vizinhança de $x$ tal que $f[V \cap A] \subset U$.

(b) Sejam $D$ espaço discreto, $p \in \beta D, X$ espaço topológico, $\left\{x_{s}: s \in D\right\} \subset X$ uma família indexada, e $y \in X$. Dizemos então que $p-\lim _{s \in D} x_{s}=y$ se e somente se para cada vizinhança $V$ de $y,\left\{s \in D: x_{s} \in V\right\} \in p$.

Teorema 1.28. (a) Se Y é Hausdorff e $\lim _{a \rightarrow x} f(a)$ existe, então é único.

(b) Se X é Hausdorff e $p-\lim _{s \in D} x_{s}$ existe, então é único.

(c) Dado $A \in p$, como identificamos $\widehat{A} \subset \beta D$ com $\beta A$, pode-se considerar $p-\lim _{s \in A} x_{s}$. Vale então que $p-\lim _{s \in A} x_{s}=y$ se e somente se $p-\lim _{s \in D} x_{s}=y$.

(d) Considerando a família indexada $\{s: s \in D\} \subset \beta D$, vale que $p-\lim _{s \in D} s=p$. 
(e) Sejam $D$ discreto, $p \in \beta D, A \in p, Y$ espaço topológico, $y \in Y$ e $f: A \rightarrow Y$. Então $p-\lim _{a \in A} f(a)=y$ se e somente se $\lim _{a \rightarrow p} f(a)=y$.

(f) Seja $X$ espaço topológico. Então $X$ é compacto se e somente se dados qualquer $D$, qualquer família indexada $\left\{x_{s}: s \in D\right\}$, e qualquer $p \in \beta D, p-\lim _{s \in D} x_{s}$ existe.

(g) Sejam $X$ espaço topológico, $A \subset X$, e $y \in X$. Então $y \in \operatorname{cl} A$ se e somente se existem $D$, $p \in \beta D e\left\{x_{s}: s \in D\right\} \subset A$ tais que $p-\lim _{s \in D} x_{s}=y$.

(h) Sejam $X, Y$ espaços topológicos, e $f: X \rightarrow Y$. Então $f$ é contínua se e somente se dados quaisquer $D, p \in \beta D$, e $\left\{x_{s}: s \in D\right\} \subset X$ tais que $p-\lim _{s \in D} x_{s}$ existe, tem-se que $f\left(p-\lim _{s \in D} x_{s}\right)=p-\lim _{s \in D} f\left(x_{s}\right)$. 


\subsection{A Compactificação de Stone-Čech de um Semigrupo Discreto}

Nesta seção apresentaremos a caracterização da operação em $\beta S$ e alguns resultados primários.

Teorema 1.29. Seja $(S, \cdot)$ semigrupo discreto. Então existe uma única operação $*: \beta S \rightarrow \beta S$ tal $\left.q u e *\right|_{S \times S}=\cdot,(\beta S, *)$ seja um semigrupo topológico à direita, e $S \subset \Lambda(\beta S)$.

Como de costume, usaremos o mesmo símbolo para denotar a operação em $\beta S$ e em $S$. Como $\beta S$ é um semigrupo topológico à direita compacto, segue que valem todas as conclusões da seção 1.2, além de, é claro, as da seção 1.3 .

Definição 1.30. Sejam $S$ semigrupo, $A \subset S$ e $s \in S$.

(a) Se a operação em $S$ for denotada por · (notação multiplicativa), então $s^{-1} A=\{t \in S: s t \in A\}$.

(b) Se a operação em $S$ for denotada por + (notação aditiva), então $-s+A=\{t \in S: s+t \in A\}$. Note que em ambos os casos esta é apenas uma notação para $\lambda_{s}{ }^{-1}[A]$.

Teorema 1.31. Sejam $(S, \cdot)$ semigrupo, $A \subset S, t \in S$ e $p, q \in \beta S$.

(a) $A \in t \cdot q$ se e somente se $t^{-1} A \in q$.

(b) $A \in p \cdot q$ se e somente se $\left\{s \in S: s^{-1} A \in q\right\} \in p$.

Seguem de (a) e (b):

(c) Se $A \in q$, então $s A \in s \cdot q$.

(d) $A \in p \cdot q$ se e somente se existem $B \in p$ e uma familia $\left\{C_{s}: s \in B\right\} \subset q$ tais que $\bigcup_{s \in B} s C_{s} \subset A$.

Além disso, se $T$ é um subsemigrupo de $S$, então podemos considerar que $\beta T$ está algebricamente imerso em $\beta S$, usando 1.25(b).

Definição 1.32. Sejam $(S, \cdot)$ semigrupo, $p \in \beta S$, e $A \subset S$. Denotamos $A^{\star}(p)=\left\{s \in A: s^{-1} A \in\right.$ $p\}$.

Segue imediatamente de 1.31(b) que $p \in \beta S$ é idempotente se e somente se para cada $A \in p$, $A^{\star}(p) \in p$. Além disso:

Teorema 1.33. Sejam $(S, \cdot)$ um semigrupo, $p \in \beta S$ idempotente, e $A \subset S$. Então para todo $s \in A^{\star}(p), s^{-1}\left(A^{\star}(p)\right) \in p$.

Teorema 1.34. Suponha que $\mathcal{A} \subset \mathcal{P}(S)$ possua a p.i.f. Se para cada $A \in \mathcal{A}$ e cada $x \in A$ existir $B \in \mathcal{A}$ tal que $x B \subset A$, então $\bigcap_{A \in \mathcal{A}} \widehat{A}$ é um subsemigrupo de $\beta S$.

Uma rápida aplicação desta condição nos dá o seguinte teorema: 
Teorema 1.35. Seja $S$ um semigrupo e seja $\left(x_{n}\right)_{n \geq 1}$ uma sequência em $S$. Então $\bigcap_{m \geq 1} \overline{F P\left(\left(x_{n}\right)_{n \geq m}\right)}$ é um subsemigrupo de $\beta S$. Em particular, existe um idempotente $p$ tal que $p \in \bigcap_{m \geq 1} \overline{F P\left(\left(x_{n}\right)_{n \geq m}\right)}$.

Teorema 1.36. Sejam $(S, \cdot)$ um semigrupo e $\mathcal{A} \subset \mathcal{P}(S)$ com a p.i.f. Sejam $(T, \cdot)$ um semigrupo topológico à direita compacto e $\phi: S \rightarrow T$ tal que $\phi[S] \subset \Lambda(T)$. Suponha que existe $A \in \mathcal{A}$ tal que para todo $x \in A$ existe $B \in \mathcal{A}$ tal que $\phi(x \cdot y)=\phi(x) \cdot \phi(y)$ para todo $y \in B$. Então, para todos $p, q \in \bigcap_{A \in \mathcal{A}} \widehat{A}, \widetilde{\phi}(p \cdot q)=\widetilde{\phi}(p) \cdot \widetilde{\phi}(q)$.

Teorema 1.37. Sejam $S$ semigrupo, $T$ semigrupo topológico à direita compacto, e $\varphi: S \rightarrow T$ um homomorfismo tal que $\varphi[S] \subset \Lambda(T)$. Então $\widetilde{\varphi}$ é um homomorfismo de $\beta S$ a T.

Teorema 1.38. Se $S$ é um semigrupo comutativo, então $S \subset Z(\beta S)=\Lambda(\beta S)$.

Definição 1.39. Seja $S$ um semigrupo.

(i) $S$ é fracamente cancelativo à esquerda se para todos $a, b \in S, \lambda_{a}{ }^{-1}[\{b\}]=\{x \in S: a x=b\}$ for finito.

(ii) $S$ é fracamente cancelativo à direita se para todos $a, b \in S, \rho_{a}{ }^{-1}[\{b\}]=\{x \in S: x a=b\}$ for finito.

Teorema 1.40. (a) Se $S$ for cancelativo à direita ou à esquerda, então $S^{*}$ é um subsemigrupo de $\beta S$.

(b) Se $S$ for fracamente cancelativo à esquerda, então $S^{*}$ é ideal à esquerda de $\beta S$, e se $S$ for cancelativo à direita, então $S^{*}$ é ideal à direita de $\beta S$.

(c) $S^{*}$ é um ideal de $\beta S$ se e somente se $S$ for fracamente cancelativo à direita e à esquerda. 


\section{Capítulo 2}

\section{A Álgebra de $\beta \mathbb{N}$}

Este capítulo se devotará a alguns resultados que nos permitirão obter um panorama das estruturas algébricas de $\beta \mathbb{N}$ e suas peculiaridades, utilizando para isso, em alguns casos, o estudo do subsemigrupo $\mathbb{H}$. A maioria resultados da primeira seção podem ser encontrados no capítulo 6 , seção 1 de [1], e serão demonstrados aqui pois já se tratam de resultados mais avançados e centrais ao tema desta dissertação.

Como convenção, sempre que for mencionado $\beta \mathbb{N}$, estará sendo considerada a estrutura algébrica da soma $(\beta \mathbb{N},+)$. Quando for utilizada a estrutura do produto $(\beta \mathbb{N}, \cdot)$, esta será explicitada.

Iniciaremos com alguns resultados encontrados no survey de Neil Hindman [3], buscando exaurir as referências usadas nas demonstrações até que estejam baseadas apenas nos resultados básicos das Preliminares.

\subsection{O Semigrupo $\mathbb{H}$}

Definição 2.1. $\mathbb{H}=\bigcap_{n \in \mathbb{N}} \overline{2^{n} \mathbb{N}}$.

Vale que $\mathbb{H}$ é um subsemigrupo, utilizando o teorema 1.34: basta notar que se $n \in 2^{k} \mathbb{N}$, então $n+2^{k} \mathbb{N} \subset 2^{k} \mathbb{N}$. Além disso, $\mathbb{H}$ contém todos os idempotentes de $\beta \mathbb{N}$, pois:

Lema 2.2. Seja $p$ um idempotente de $\beta \mathbb{N}$. Então, para todo $n \in \mathbb{N}, n \mathbb{N} \in p$.

Demonstração. Considere $\varphi: \mathbb{N} \rightarrow \mathbb{Z}_{n}$ o homomorfismo canônico. Então, pelo teorema 1.37, tem-se que $\widetilde{\varphi}: \beta \mathbb{N} \rightarrow \mathbb{Z}_{n}$ é um homomorfismo. Assim, $\widetilde{\varphi}(p)=\widetilde{\varphi}(p+p)=\widetilde{\varphi}(p)+\widetilde{\varphi}(p)$, $\operatorname{logo} \widetilde{\varphi}(p)=\overline{0}$. Portanto, $\{\overline{0}\}$ é uma vizinhança de $\widetilde{\varphi}(p)$, e assim $\widetilde{\varphi}^{-1}[\{\overline{0}\}] \cap \mathbb{N}=n \mathbb{N} \in p$.

Definição 2.3. (a) Dado $n \in \mathbb{N}$, define-se $\operatorname{supp}(n) \in \mathcal{P}_{f}(\omega)$ por $n=\sum_{i \in \operatorname{supp}(n)} 2^{i}$. Note que está bem definido, pois se trata da expansão binária de $n$.

(b) $\phi: \mathbb{N} \rightarrow \omega$ é definida por $\phi(n)=\max (\operatorname{supp}(n))$. 
(c) $\theta: \mathbb{N} \rightarrow \omega$ é definida por $\theta(n)=\min (\operatorname{supp}(n))$.

Lema 2.4. $\mathbb{H}$ é um subsemigrupo compacto de $\beta \mathbb{N}$ que possui todos os idempotentes. Além disso, dados $p \in \beta \mathbb{N}$ e $q \in \mathbb{H}, \widetilde{\phi}(p+q)=\widetilde{\phi}(q)$ e $\widetilde{\theta}(p+q)=\widetilde{\theta}(p)$.

Demonstração. Já vimos que $\mathbb{H}$ é um subsemigrupo que possui todos os idempotentes. Ele é claramente compacto.

Sejam $p \in \beta \mathbb{N}$ e $q \in \mathbb{H}$. Dado $m \in \mathbb{N}$, tome $r>\phi(m)$. Então, para todo $n \in 2^{r} \mathbb{N}, \phi(m+n)=\phi(n)$ e $\theta(m+n)=\theta(m)$. Logo, como $2^{r} \mathbb{N} \in q$,

$$
\begin{aligned}
\widetilde{\phi}(m+q) & =q-\lim _{n \in 2^{r} \mathbb{N}} \phi(m+n) \\
& =q-\lim _{n \in 2^{r} \mathbb{N}} \phi(n) \\
& =\widetilde{\phi}(q) .
\end{aligned}
$$

Dessa forma, $\widetilde{\phi}(p+q)=\lim _{m \rightarrow p} \widetilde{\phi}(m+q)=\widetilde{\phi}(q)$.

Analogamente, $\widetilde{\theta}(p+q)=\widetilde{\theta}(p)$.

Com esse lema, provaremos dois fatos fundamentais sobre $\beta \mathbb{N}$. O primeiro é este:

Teorema 2.5. $\beta \mathbb{N}$ contém $2^{\mathfrak{c}}$ ideais à esquerda minimais e $2^{\mathfrak{c}}$ ideais à direita minimais. Cada um possui $2^{\mathfrak{c}}$ idempotentes.

Demonstração. Considere $A=\left\{2^{n}: n \in \mathbb{N}\right\}$. Note que $A^{*} \subset \mathbb{H}$. Como $\phi\left(2^{n}\right)=\theta\left(2^{n}\right)=n$ para todo $n \in \mathbb{N},\left.\phi\right|_{A}=\left.\theta\right|_{A}: A \rightarrow \mathbb{N}$ é bijetora, $\left.\log 0 \widetilde{\phi}\right|_{\bar{A}}=\left.\widetilde{\theta}\right|_{\bar{A}}: \bar{A} \rightarrow \beta \mathbb{N}$ é bijetora. Agora, suponha que $q_{1}, q_{2} \in A^{*}$ e que $\left(\beta \mathbb{N}+q_{1}\right) \cap\left(\beta \mathbb{N}+q_{2}\right) \neq \varnothing$. Então existem $r, s \in \beta \mathbb{N}$ tais que $r+q_{1}=s+q_{2}$, $\operatorname{logo} \widetilde{\phi}\left(q_{1}\right)=\widetilde{\phi}\left(r+q_{1}\right)=\widetilde{\phi}\left(s+q_{2}\right)=\widetilde{\phi}\left(q_{2}\right)$, do que $q_{1}=q_{2}$. Como, por $1.26(\mathrm{~d}),\left|A^{*}\right|=2^{\mathfrak{c}}$, segue que $\beta \mathbb{N}$ possui $2^{\mathfrak{c}}$ ideais à esquerda minimais disjuntos.

Agora note que como $\mathbb{H}$ é um subsemigrupo que possui todos os idempotentes de $\beta \mathbb{N}$, qualquer idempotente que for minimal em $\mathbb{H}$ o será em $\beta \mathbb{N}$. Assim, dado $q \in A^{*}$, pelos teoremas 1.15 e 1.16 , $q+\mathbb{H}$ possui um idempotente $e(q)$ que é minimal em $\mathbb{H}$, e logo minimal em $\beta \mathbb{N}$, e portanto $e(q)+\beta \mathbb{N}$ é um ideal à direita minimal de $\beta \mathbb{N}$.

Afirmação: Se $q_{1}, q_{2} \in A^{*}$ são distintos, então $e\left(q_{1}\right)+\beta \mathbb{N} \neq e\left(q_{2}\right)+\beta \mathbb{N}$. Provaremos por contrapositiva. Suponha $e\left(q_{1}\right)+\beta \mathbb{N}=e\left(q_{2}\right)+\beta \mathbb{N}$. Então $e\left(q_{2}\right) \in e\left(q_{1}\right)+\beta \mathbb{N}, \operatorname{logo} e\left(q_{2}\right)=e\left(q_{1}\right)+e\left(q_{2}\right)$, e portanto $\widetilde{\theta}\left(e\left(q_{2}\right)\right)=\widetilde{\theta}\left(e\left(q_{1}\right)+e\left(q_{2}\right)\right)=\widetilde{\theta}\left(e\left(q_{1}\right)\right)$. Porém, $\widetilde{\theta}\left(e\left(q_{2}\right)\right) \in \widetilde{\theta}\left[q_{2}+\mathbb{H}\right]=\left\{q_{2}\right\}$ e $\widetilde{\theta}\left(e\left(q_{1}\right)\right) \in$ $\widetilde{\theta}\left[q_{1}+\mathbb{H}\right]=\left\{q_{1}\right\}$, pelo lema 2.4. Assim, $q_{1}=q_{2}$. Dessa forma, $\beta \mathbb{N}$ possui $2^{\mathfrak{c}}$ ideais à direita minimais. Como todo ideal à esquerda (ou à direita) contém um ideal à esquerda (à direita) minimal (1.15(a)); como dois ideais à esquerda (ou à direita) minimais distintos são disjuntos; e como dados $L$ ideal à esquerda minimal e $R$ ideal à direita minimal quaisquer, $L \cap R$ possui um idempotente (1.17(b)); segue que tanto $L$ como $R$ possuem $2^{\mathfrak{c}}$ idempotentes (cada um deles minimal pelo teorema 1.16). 
Note que, se um semigrupo $S$ possui dois ideais à esquerda disjuntos $L_{1}$ e $L_{2}$, então ele não é comutativo - dados $l_{1} \in L_{1}$ e $l_{2} \in L_{2}, l_{2} l_{1} \in L_{1}$ e $l_{1} l_{2} \in L_{2}$. Assim, o que o teorema acima diz, de certa forma, é que $\beta \mathbb{N}$ está "bem longe" de ser comutativo. De fato, seguindo nesta mesma ideia, o próximo fato fundamental sobre $\beta \mathbb{N}$ é este:

Teorema 2.6. $\mathbb{N}$ é o centro de $(\beta \mathbb{N},+)$ e de $(\beta \mathbb{N}, \cdot)$.

Demonstração. Pelo teorema 1.38, $\mathbb{N}$ está contido nos centros de $(\beta \mathbb{N},+)$ e de $(\beta \mathbb{N}, \cdot)$.

Considere $A=\left\{2^{n}: n \in \mathbb{N}\right\}$. Sejam $p \in \mathbb{N}^{*}$ e $q \in A^{*}$. Pelo lema 2.4, $\widetilde{\phi}(p+q)=\widetilde{\phi}(q)$. Por outro lado, dados $m, n \in \mathbb{N}$ tais que $n>m$, temos que $\phi(m+n)=\phi(n)$ ou $\phi(m+n)=\phi(n)+1$. Para cada $m \in \mathbb{N},\{n \in \mathbb{N}: \phi(m+n)=\phi(n)\} \in p$ ou $\{n \in \mathbb{N}: \phi(m+n)=\phi(n)+1\} \in p$ (pois $\left.p \in \mathbb{N}^{*}\right)$. No primeiro caso, temos que $p \in \operatorname{cl}\{n \in \mathbb{N}: \phi(m+n)=\phi(n)\}, \operatorname{logo} \widetilde{\phi}(m+p)=\widetilde{\phi}(p)$. No segundo caso, $\widetilde{\phi}(m+p)=\widetilde{\phi}(p)+1$. Temos também que $\{m \in \mathbb{N}: \widetilde{\phi}(m+p)=\widetilde{\phi}(p)\} \in q$ ou que $\{m \in \mathbb{N}: \widetilde{\phi}(m+p)=\widetilde{\phi}(p)+1\} \in q$. Portanto, $\widetilde{\phi}(q+p)=\widetilde{\phi}(p)$ ou $\widetilde{\phi}(q+p)=\widetilde{\phi}(p)+1$. Como $\left.\widetilde{\phi}\right|_{\bar{A}}$ é bijetora e $\left|A^{*}\right|=2^{\mathfrak{c}}$, então podemos tomar $q \in A^{*}$ tal que $\widetilde{\phi}(q) \notin\{\widetilde{\phi}(p), \widetilde{\phi}(p)+1\}$. Assim, $\widetilde{\phi}(p+q)=\widetilde{\phi}(q) \neq \widetilde{\phi}(q+p)$. Destarte, $p+q \neq q+p$ e logo $p \notin Z((\beta \mathbb{N},+))$.

Agora, dados $m, n \in \mathbb{N}, \phi\left(m \cdot 2^{n}\right)=\phi(m)+n=\phi(m)+\phi\left(2^{n}\right)$. Portanto, dados $p \in \mathbb{N}^{*}$ e $q \in A^{*}$, temos:

$$
\begin{aligned}
\widetilde{\phi}(p \cdot q) & =\lim _{m \rightarrow p} \lim _{2^{n} \rightarrow q} \phi\left(m \cdot 2^{n}\right) \\
& =\lim _{m \rightarrow p} \lim _{2^{n} \rightarrow q}\left(\phi(m)+\phi\left(2^{n}\right)\right) \\
& =\lim _{m \rightarrow p}(\phi(m)+\widetilde{\phi}(q)) \\
& =\widetilde{\phi}(p)+\widetilde{\phi}(q) .
\end{aligned}
$$

De forma completamente análoga, $\widetilde{\phi}(q \cdot p)=\widetilde{\phi}(q)+\widetilde{\phi}(p)$.

Agora, pelo $1.26(\mathrm{e})$, como $\phi$ é finita-por-unitários, $\widetilde{\phi}(p) \in \mathbb{N}^{*}$, logo, como acabamos de provar, $\widetilde{\phi}(p) \notin Z((\beta \mathbb{N},+))$, logo tome $r \in \mathbb{N}^{*}$ tal que $\widetilde{\phi}(p)+r \neq r+\widetilde{\phi}(p)$. Novamente, como $\left.\widetilde{\phi}\right|_{\bar{A}}$ é bijetora, seja $q \in A^{*}$ tal que $\widetilde{\phi}(q)=r$. Então temos que $\widetilde{\phi}(p \cdot q)=\widetilde{\phi}(p)+\widetilde{\phi}(q)=\widetilde{\phi}(p)+r \neq r+\widetilde{\phi}(p)=$ $\widetilde{\phi}(q)+\widetilde{\phi}(p)=\widetilde{\phi}(q \cdot p)$, e assim $p \cdot q \neq q \cdot p$. Ou seja $p \notin Z((\beta \mathbb{N}, \cdot))$.

Note que disso segue que, pelo teorema $1.38, \mathbb{N}=Z((\beta \mathbb{N},+))=\Lambda((\beta \mathbb{N},+))$ e $\mathbb{N}=Z((\beta \mathbb{N}, \cdot))=$ $\Lambda((\beta \mathbb{N}, \cdot))$.

Agora investigaremos propriedades que tornam o semigrupo $\mathbb{H}$ interessante. O primeiro deles foi extraído da página 110 de [1]. Mas antes precisaremos do seguinte lema (cujo enunciado é um exercício encontrado na página 79):

Lema 2.7. Sejam $(S, \cdot)$ um semigrupo, $k \in \mathbb{N} \backslash\{1\}$, e $p_{1}, \ldots, p_{k} \in \beta S$. Considere $D \subset\{\varnothing\} \cup$ $\bigcup_{i=1}^{k-1} S^{i}$, com $\varnothing \in D$. Suponha que, para cada $\sigma \in D$, há um $A_{\sigma} \subset S$ de forma que: 
(1) $A_{\varnothing} \in p_{1}$;

(2) $A_{\varnothing} \subset D$;

(3) $A_{\sigma} \in p_{i+1}$ se $\sigma \in D \cap S^{i}$;

(4) se $k>2$, então para cada $i \in\{1, \ldots, k-2\}$ e cada $\sigma=\left(a_{1}, \ldots, a_{i-1}\right) \in D$, se a $\in A_{\sigma}$, então $\left(a_{1}, \ldots, a_{i-1}, a\right) \in D$.

Considere $P=\left\{a_{1} \cdot \ldots \cdot a_{k}: a_{1} \in A_{\varnothing}\right.$ e, para cada $i \in\{2, \ldots, k\},\left(a_{1}, \ldots, a_{i-1}\right) \in D$ e $a_{i} \in$ $\left.A_{\left(a_{1}, \ldots, a_{i-1}\right)}\right\}$.

Então $P \in p_{1} \cdot \ldots p_{k}$.

Demonstração. Provaremos por indução.

Caso base: $k=2$. Temos que $P=\left\{a_{1} \cdot a_{2}: a_{1} \in A_{\varnothing}, a_{1} \in D\right.$ e $\left.a_{2} \in A_{a_{1}}\right\}$. Queremos ver que $P \in p_{1} \cdot p_{2}$, ou seja, que $\left\{s \in S: s^{-1} P \in p_{2}\right\} \in p_{1}$. Como $A_{\varnothing} \in p_{1}$, basta ver que $A_{\varnothing} \subset\{s \in S$ : $\left.s^{-1} P \in p_{2}\right\}$. Logo seja $a_{1} \in A_{\varnothing}$. Queremos ver que $a_{1}{ }^{-1} P \in p_{2}$. Como $A_{a_{1}} \in p_{2}$, basta ver que $A_{a_{1}} \subset a_{1}{ }^{-1} P$. Logo seja $a_{2} \in A_{a_{1}}$. Note que $a_{1} \cdot a_{2} \in P$, como desejado.

Passo indutivo: $k>2$. Tome $P$ como no enunciado. Queremos ver que $\left\{s \in S: s^{-1} P \in p_{k}\right\} \in p_{1} \ldots$. $p_{k-1}$. Pela hipótese indutiva, $\left\{a_{1} \cdot \ldots \cdot a_{k-1}: a_{1} \in A_{\varnothing}\right.$ e, para cada $i \in\{2, \ldots, k-1\},\left(a_{1}, \ldots, a_{i-1}\right) \in$ $D$ e $\left.a_{i} \in A_{\left(a_{1}, \ldots, a_{i-1}\right)}\right\} \in p_{1} \cdot \ldots \cdot p_{k-1}$. Logo basta ver que $\left\{a_{1} \cdot \ldots \cdot a_{k-1}: a_{1} \in A_{\varnothing}\right.$ e, para cada $i \in\{2, \ldots, k-1\},\left(a_{1}, \ldots, a_{i-1}\right) \in D$ e $\left.a_{i} \in A_{\left(a_{1}, \ldots, a_{i-1}\right)}\right\} \subset\left\{s \in S: s^{-1} P \in p_{k}\right\}$. Logo seja $a_{1} \cdot \ldots \cdot a_{k-1}$ tal que $a_{1} \in A_{\varnothing}$ e, para cada $i \in\{2, \ldots, k-1\},\left(a_{1}, \ldots, a_{i-1}\right) \in D$ e $a_{i} \in A_{\left(a_{1}, \ldots, a_{i-1}\right)}$. Note que, em particular, $\left(a_{1}, \ldots, a_{k-2}\right) \in D$ e $a_{k-1} \in A_{\left(a_{1}, \ldots, a_{k-2}\right)}$, e portanto $\left(a_{1}, \ldots, a_{k-1}\right) \in D$. Queremos ver que $a_{1} \cdot \ldots \cdot a_{k-1}{ }^{-1} P \in p_{k}$. Sabemos que $A_{\left(a_{1}, \ldots, a_{k-1}\right)} \in p_{k}$. Logo basta ver que $A_{\left(a_{1}, \ldots, a_{k-1}\right)} \subset a_{1} \cdot \ldots \cdot a_{k-1}{ }^{-1} P$. Seja $a \in A_{\left(a_{1}, \ldots, a_{k-1}\right)}$. Note que $a$ satisfaz os requisitos necessários de forma que $a_{1} \cdot \ldots \cdot a_{k-1} \cdot a \in P$, como desejávamos.

Teorema 2.8. $\mathbb{H}$ contém uma sequência infinita decrescente de idempotentes.

Demonstração. Tome $\left(A_{n}\right)_{n \geq 1}$ uma sequência crescente de subconjuntos de $\mathbb{N}$ tal que $A_{n+1} \backslash A_{n}$ é infinito para cada $n \geq 1$. Considere, para cada $n, S_{n}=\left\{m \in \mathbb{N}: \operatorname{supp}(m) \subset A_{n}\right\}$. Note que, para cada $n, r \in \mathbb{N}$, se $k, m \in 2^{r} \mathbb{N} \cap S_{n}$ e $\operatorname{supp}(k) \cap \operatorname{supp}(m)=\varnothing$, então $k+m \in 2^{r} \mathbb{N} \cap S_{n}$. Logo, pelo teorema 1.34, $T_{n}=\overline{S_{n}} \cap \mathbb{H}$ é um subsemigrupo de $\beta \mathbb{N}$ (usando $\mathcal{A}=\left\{S_{n} \cap 2^{r} \mathbb{N}: r \in \mathbb{N}\right\}$ ). Note que $T_{n} \subset T_{n+1}$, pois $S_{n} \subset S_{n+1}$, para todo $n \geq 1$. A partir disso, construiremos uma sequência injetora $\left(e_{n}\right)_{n \geq 1}$ de idempotentes tal que $e_{n+1} \leq e_{n}$ e $e_{n} \in K\left(T_{n}\right)$ para todo $n \geq 1$.

Tome $e_{1}$ qualquer idempotente minimal em $T_{1}$. Suponha recursivamente agora que $\left\{e_{1}, \ldots, e_{m}\right\}$ já foi escolhido. Pelo $1.17(\mathrm{a})$, existe $e_{m+1} \in K\left(T_{m+1}\right)$ tal que $e_{m+1} \leq e_{m}$. Mostraremos que $e_{m} \notin K\left(T_{m+1}\right)$, do que $e_{m} \neq e_{m+1}$. 
Para ver isso, tome $x \in \mathbb{N}^{*} \cap \overline{\left\{2^{n}: n \in A_{m+1} \backslash A_{m}\right\}}$ qualquer. Considere $M=\left\{r+2^{n}+s: r, s \in\right.$ $\mathbb{N}, n \in A_{m+1} \backslash A_{m}$ e $\left.\max (\operatorname{supp}(r))<n<\min (\operatorname{supp}(s))\right\}$. Observe que para cada $r+2^{n}+s \in M$, $n \in \operatorname{supp}\left(r+2^{n}+s\right) \backslash A_{m}, \operatorname{logo} r+2^{n}+s \notin S_{m}$. Ou seja, $M \cap S_{m}=\varnothing$, e $\operatorname{logo} e_{m} \notin \bar{M}$. Agora, para ver que $e_{m} \notin K\left(T_{m+1}\right)$, veremos que $\bar{M} \supset K\left(T_{m+1}\right)$. Para isso, mostraremos que $\bar{M}$ contém o ideal $T_{m+1}+x+T_{m+1}$ de $T_{m+1}$. Com este fim, mostraremos que, para todos $y, z \in \mathbb{H}, y+x+z \in \bar{M}$. Assim, queremos ver que $M \in y+x+z$. Usaremos o lema 2.7. Considere $D=\{\varnothing\} \cup \mathbb{N} \cup\left\{\left(r, 2^{n}\right)\right.$ : $r \in \mathbb{N}, n \in A_{m+1} \backslash A_{m}$ e $\left.\max (\operatorname{supp}(r))<n\right\}$. Defina $A_{\varnothing}=\mathbb{N}, A_{r}=\left\{2^{n}: n \in A_{m+1} \backslash A_{m}\right.$ e $\max (\operatorname{supp}(r))<n\}$ para cada $r \in \mathbb{N}$, e $A_{\left(r, 2^{n}\right)}=\{s \in \mathbb{N}: n<\min (\operatorname{supp}(s))\}$, para cada $\left(r, 2^{n}\right) \in \mathbb{N}^{2}$ tal que $n \in A_{m+1} \backslash A_{m}$ e $\max (\operatorname{supp}(r))<n$. Deste modo, temos que: $A_{\varnothing} \in y ; A_{\varnothing} \subset D$; para cada $r \in D \cap \mathbb{N}$, como $x \in \mathbb{N}^{*} \cap \overline{\left\{2^{n}: n \in A_{m+1} \backslash A_{m}\right\}}, A_{r} \in x$; para cada $\left(r, 2^{n}\right) \in D \cap \mathbb{N}^{2}$, como $z \in \mathbb{H} \subset \mathbb{N}^{*}, A_{\left(r, 2^{n}\right)} \in z$; e por fim, dado $r \in D$, se $2^{n} \in A_{r}$, então $\left(r, 2^{n}\right) \in D$. Portanto podemos de fato aplicar o lema 2.7 e obter que $M \in y+x+z$.

A segunda propriedade é o Teorema 3.5 de [3].

Teorema 2.9. O semigrupo $\mathbb{H}$ é a união disjunta de $\mathfrak{c}$ ideais à direita compactos.

Demonstração. Seja $\left\{E_{\sigma}: 0<\sigma<\mathfrak{c}\right\}$ uma família quase disjunta de subconjuntos de $\omega$. Para cada $\sigma$, defina $B_{\sigma}=\left\{\sum_{n \in F} 2^{n}: F \in \mathcal{P}_{f}(\omega)\right.$ e min $\left.F \in E_{\sigma}\right\}$. Defina então $R_{\sigma}=\mathbb{H} \cap \overline{B_{\sigma}}$, e $R_{0}=\mathbb{H} \backslash \bigcup\left\{R_{\sigma}: 0<\sigma<\mathfrak{c}\right\}$. Vale que para cada $0<\sigma<\mathfrak{c}, R_{\sigma}$ é aberto e fechado em $\mathbb{H}$, logo $R_{\sigma}$ é compacto para cada $\sigma<\mathfrak{c}$.

Agora, vale que $\left\{\mathbb{N} \backslash B_{\sigma}: 0<\sigma<\mathfrak{c}\right\} \cup\left\{2^{n} \mathbb{N}: n \in \mathbb{N}\right\}$ tem a p.i.f. Para ver isso, como $\left\{2^{n} \mathbb{N}: n \in \mathbb{N}\right\}$ tem a p.i.f., basta ver que dados $0<\sigma_{1}, \ldots, \sigma_{k}<\mathfrak{c}$ e $m \in \mathbb{N},\left(\mathbb{N} \backslash B_{\sigma_{1}}\right) \cap \ldots \cap\left(\mathbb{N} \backslash B_{\sigma_{k}}\right) \cap 2^{m} \mathbb{N} \neq$ $\varnothing$. Para isso, tome $\delta \in \mathfrak{c} \backslash\left\{0, \sigma_{1}, \ldots, \sigma_{k}\right\}$ e seja $l \in \omega$ tal que $E_{\delta} \cap E_{\sigma_{i}} \subset\{0, \ldots, l\}$, para cada $i \in\{1, \ldots, k\}$. Assim, tome $F \in \mathcal{P}_{f}(\omega)$ tal que min $F \in E_{\delta}$ e min $F>l, m$. Então temos que $\sum_{n \in F} 2^{n} \in\left(\mathbb{N} \backslash B_{\sigma_{1}}\right) \cap \ldots \cap\left(\mathbb{N} \backslash B_{\sigma_{k}}\right) \cap 2^{m} \mathbb{N}$, como queríamos.

Logo, se $x \in \bigcap\left(\left\{\overline{\mathbb{N} \backslash B_{\sigma}}: 0<\sigma<\mathfrak{c}\right\} \cup\left\{\overline{2^{n} \mathbb{N}}: n \in \mathbb{N}\right\}\right)$, então $x \in R_{0}$. Portanto $R_{0} \neq \varnothing$. Além disso, dados $0<\sigma, \tau<\mathfrak{c}$ distintos, tome $n \in \mathbb{N}$ tal que $E_{\sigma} \cap E_{\tau} \subset\{0, \ldots, n-1\}$. Então, se $\sum_{n \in F} 2^{n} \in B_{\sigma} \cap B_{\tau}$, min $F \in\{0, \ldots, n-1\}$; ou seja, $B_{\sigma} \cap B_{\tau} \cap 2^{n} \mathbb{N}=\varnothing$. Destarte, $R_{\sigma} \cap R_{\tau}=\varnothing$ (pois se $x \in R_{\sigma} \cap R_{\tau}$, então $B_{\sigma} \cap B_{\tau} \cap 2^{n} \mathbb{N} \in x$ ). Temos então que $\left\{R_{\sigma}: \sigma<\mathfrak{c}\right\}$ é realmente uma coleção de compactos dois-a-dois disjuntos.

Agora veremos que $R_{\sigma}$ é um ideal à direita de $\mathbb{H}$. Sejam $q \in R_{\sigma}$ e $p \in \mathbb{H}$. $\mathbb{H}$ é subsemigrupo, logo $q+p \in \mathbb{H}$.

Primeiramente, suponha que $\sigma>0$. Queremos ver que $B_{\sigma} \in q+p$, ou seja, $\left\{x \in \mathbb{N}:-x+B_{\sigma} \in p\right\} \in$ q. Então basta ver que $B_{\sigma} \subset\left\{x \in \mathbb{N}:-x+B_{\sigma} \in p\right\}$. Logo seja $x=\sum_{n \in F} 2^{n}$ tal que $F \in \mathcal{P}_{f}(\omega)$ e min $F \in E_{\sigma}$. Tome $m=\min F+1$. Então, dado qualquer $y \in 2^{m} \mathbb{N}, \min (\operatorname{supp}(x+y))=\min F$, $\operatorname{logo} x+y \in B_{\sigma}$. Isto é, $2^{m} \mathbb{N} \subset-x+B_{\sigma}$. Como $2^{m} \mathbb{N} \in p$, segue a tese. 
Por fim, temos o caso em que $\sigma=0$. Suponha por absurdo que $q+p \notin R_{0}$, ou seja, $q+p \in R_{\tau}$ para algum $\tau>0$. Então $B_{\tau} \in q+p$, ou seja, $\left\{x \in \mathbb{N}:-x+B_{\tau} \in p\right\} \in q$. Como $B_{\tau} \notin q$, então tome $x \in \mathbb{N}$ tal que $x \notin B_{\tau}$ e $-x+B_{\tau} \in p$. Tome $F \in \mathcal{P}_{f}(\omega)$ tal que $\min F \notin E_{\tau}$ e $x=\sum_{n \in F} 2^{n}$. Seja $m=\min F+1.2^{m} \mathbb{N} \cap\left(-x+B_{\tau}\right) \in p$, logo tome $y$ nessa interseção. Então $x+y \in B_{\tau}$. Porém, como acima, note que $\min (\operatorname{supp}(x+y))=\min F \notin E_{\tau}$, e portanto $x+y \notin B_{\tau}$. Contradição. Deste modo, $q+p \in R_{0}$.

Agora veremos que cópias algébricas e topológicas de $\mathbb{H}$ são abundantes em $\mathbb{N}^{*}$.

Teorema 2.10. Seja $\left(x_{n}\right)_{n \geq 1}$ uma sequência em $\mathbb{N}$ com a unicidade das somas finitas. Então $\bigcap_{m \geq 1} \overline{F S\left(\left(x_{n}\right)_{n \geq m}\right)}$ é algébrica e topologicamente isomorfo a $\mathbb{H}$, pela mesma função.

Demonstração. Defina $f: \mathbb{N} \rightarrow \mathbb{N}$ por $f\left(\sum_{n \in F} 2^{n-1}\right)=\sum_{n \in F} x_{n}$. Note que de fato $f$ está bemdefinida sobre $\mathbb{N}$ e, como $\left(x_{n}\right)_{n \geq 1}$ satisfaz a unicidade das somas finitas, $f$ é injetora. Considere $\tilde{f}: \beta \mathbb{N} \rightarrow \beta \mathbb{N}$ a extensão contínua de $f$ e defina $\varphi=\left.\widetilde{f}\right|_{\mathbb{H}}$. Observe que, para cada $m \in \mathbb{N}$, $f\left[2^{m-1} \mathbb{N}\right]=F S\left(\left(x_{n}\right)_{n \geq m}\right), \operatorname{logo} \tilde{f}\left[\overline{2^{m-1} \mathbb{N}}\right]=\overline{F S\left(\left(x_{n}\right)_{n \geq m}\right)}$. Assim - usando que $\tilde{f}$ é injetora $\varphi[\mathbb{H}]=\widetilde{f}[\mathbb{H}]=\bigcap_{m \in \mathbb{N}} \tilde{f}\left[\overline{2^{m-1} \mathbb{N}}\right]=\bigcap_{m \in \mathbb{N}} \overline{F S\left(\left(x_{n}\right)_{n \geq m}\right)}$. Assim, $\varphi$ é uma bijeção contínua entre $\mathbb{H}$ e $\bigcap_{m \in \mathbb{N}} \overline{F S\left(\left(x_{n}\right)_{n \geq m}\right)}$; como ambos são compactos Hausdorff, é um homeomorfismo.

Resta ver que $\varphi$ é um homomorfismo. Para isso, lembraremos que $\beta \mathbb{N}$ é um semigrupo topológico à direita e que $\mathbb{N}=\Lambda(\beta \mathbb{N})$. Sejam $p, q \in \mathbb{H}$. Mostraremos que $\varphi(p+q)=\varphi(p)+\varphi(q)$, ou seja, que $\varphi \circ \rho_{q}(p)=\rho_{\varphi(q)} \circ \varphi(p)$. Basta ver que estas duas funções contínuas são idênticas sobre $\mathbb{N}$. Seja então $x \in \mathbb{N}$. Quero ver que $\varphi(x+q)=\varphi(x)+\varphi(q)$, ou seja, que $\varphi \circ \lambda_{x}(q)=\lambda_{\varphi(x)} \circ \varphi(q)$. Tome $F \in \mathcal{P}_{f}(\mathbb{N})$ tal que $x=\sum_{n \in F} 2^{n-1}$, e seja $m=\max F$. Como $q \in \overline{2^{m} \mathbb{N}}$, basta ver que $\varphi \circ \lambda_{x}$ e $\lambda_{\varphi(x)} \circ \varphi$ são idênticas sobre $2^{m} \mathbb{N}$. Seja $y \in 2^{m} \mathbb{N}$, e tome $G \in \mathcal{P}_{f}(\mathbb{N})$ tal que $y=\sum_{n \in G} 2^{n-1}$. Note que $\min G>m$, e portanto $x+y=\sum_{n \in F \cup G} 2^{n-1}$. Assim, $\varphi \circ \lambda_{x}(y)=\varphi(x+y)=f(x+$ $y)=f\left(\sum_{n \in F \cup G} 2^{n-1}\right)=\sum_{n \in F \cup G} x_{n}=\sum_{n \in F} x_{n}+\sum_{n \in G} x_{n}=f\left(\sum_{n \in F} 2^{n-1}\right)+f\left(\sum_{n \in G} 2^{n-1}\right)=$ $f(x)+f(y)=\varphi(x)+\varphi(y)=\lambda_{\varphi(x)} \circ \varphi(y)$. 


\subsection{O Ideal $\mathbb{N}^{*}+\mathbb{N}^{*}$, o Fecho de $K(\beta \mathbb{N}), \mathcal{U}_{\kappa}(S)$, Somas e Produtos}

O ponto final desta seção é uma consequência do Teorema 3.7 de [3]: embora $\mathbb{N}^{*}+\mathbb{N}^{*}$ seja um ideal de $\beta \mathbb{N}$, e portanto $K(\beta \mathbb{N}) \subset \mathbb{N}^{*}+\mathbb{N}^{*}$, tem-se que $\operatorname{cl} K(\beta \mathbb{N}) \not \subset \mathbb{N}^{*}+\mathbb{N}^{*}$. Para isso, precisaremos de resultados sobre a álgebra de $\mathcal{U}_{\kappa}(S)$, e também sobre as relações entre somas e produtos em $\beta \mathbb{N}$ - ambos assuntos interessantes por si e com consequências em outros resultados.

Lema 2.11. $\mathbb{N}^{*}$ é um ideal à esquerda de $\beta \mathbb{Z}$.

Demonstração. Sejam $p \in \mathbb{N}^{*}$ e $q \in \beta \mathbb{Z}$. Queremos ver que $q+p \in \overline{\mathbb{N}}$ e que $q+p \notin \mathbb{N}$. Suponha primeiramente que $q+p=a \in \mathbb{N}$. Ou seja, $q+p=\{A \subset \mathbb{Z}: a \in A\}$. Em particular, $\{a\} \in q+p$, ou seja, $\{x \in \mathbb{Z}:-x+\{a\} \in p\} \in q$, e portanto existe $x \in \mathbb{Z}$ tal que $-x+\{a\}=\{-x+a\} \in p$. Contradição, pois $p \in \mathbb{N}^{*}$.

Resta ver que $\{x \in \mathbb{Z}:-x+\mathbb{N} \in p\} \in q$. Veremos que $\{x \in \mathbb{Z}:-x+\mathbb{N} \in p\}=\mathbb{Z}$ (note que esta é a única estratégia, pois $q$ é arbitrário em $\beta \mathbb{Z}$ ). Logo tome $x \in \mathbb{Z}$ e suponha que $-x+\mathbb{N} \notin p$. Então $-x+\mathbb{Z} \backslash \mathbb{N} \in p, \operatorname{logo} \mathbb{N} \cap(-x+\mathbb{Z} \backslash \mathbb{N}) \in p$, porém $\mathbb{N} \cap(-x+\mathbb{Z} \backslash \mathbb{N})=\{n \in \mathbb{N}: n \leq-x\}$ que é finito. Contradição pois $p \in \mathbb{N}^{*}$.

Corolário 2.12. $\mathbb{N}^{*}+\mathbb{N}^{*}$ é ideal de $\beta \mathbb{N}$.

Demonstração. Pelo lema 2.11, temos que:

$\beta \mathbb{N}+\left(\mathbb{N}^{*}+\mathbb{N}^{*}\right)=\left(\beta \mathbb{N}+\mathbb{N}^{*}\right)+\mathbb{N}^{*} \subset \mathbb{N}^{*}$. Por outro lado, $\left(\mathbb{N}^{*}+\mathbb{N}^{*}\right)+\mathbb{N}^{*} \subset \mathbb{N}^{*}$, e, como $\mathbb{N} \subset Z(\beta \mathbb{N})$, $\left(\mathbb{N}^{*}+\mathbb{N}^{*}\right)+\mathbb{N}=\mathbb{N}+\left(\mathbb{N}^{*}+\mathbb{N}^{*}\right)=\left(\mathbb{N}+\mathbb{N}^{*}\right)+\mathbb{N}^{*} \subset \mathbb{N}^{*} ;$ portanto $\left(\mathbb{N}^{*}+\mathbb{N}^{*}\right)+\beta \mathbb{N} \subset \mathbb{N}^{*}$

(Observe que, com um raciocínio ainda mais simples, $\mathbb{N}^{*}$ é um ideal de $\beta \mathbb{N}$ também.)

Note, pelo teorema 1.16, que $\{p \in \beta \mathbb{N}: p$ é idempotente minimal $\} \subset K(\beta \mathbb{N})$. Então, mais forte que $\operatorname{cl} K(\beta \mathbb{N}) \not \subset \mathbb{N}^{*}+\mathbb{N}^{*}$, teremos que $\operatorname{cl}\{p \in \beta \mathbb{N}: p$ é idempotente minimal $\} \not \subset \mathbb{N}^{*}+\mathbb{N}^{*}$. Enunciemos e provemos então o Teorema 3.7 de [3]:

Teorema 2.13. Seja $M=\operatorname{cl}\{p \in \beta \mathbb{N}: p$ é idempotente minimal de $(\beta \mathbb{N},+)\}$. Então $M \backslash\left(\mathbb{N}^{*}+\mathbb{N}^{*}\right) \neq$ $\varnothing$.

Demonstração. Pelo Teorema 5.4 de [5], $M$ é um ideal à esquerda de $(\beta \mathbb{N}, \cdot)$. Em particular, é um subsemigrupo compacto, logo tome $q \in M$ tal que $q=q \cdot q$. Pelo lema 2.2 , temos que $\{p \in \beta \mathbb{N}: p$ é idempotente minimal $\} \subset \bigcap_{n \in \mathbb{N}} \overline{n \mathbb{N}}$, $\log 0 \quad M \subset \bigcap_{n \in \mathbb{N}} \overline{n \mathbb{N}}$. Assim, $n \mathbb{N} \in q$, para todo $n \in \mathbb{N}$. Então, pelo Teorema 5.3 de $[6], q=q \cdot q \notin \mathbb{N}^{*}+\mathbb{N}^{*}$.

Nosso objetivo é agora chegar aos resultados citados, mas não provados, no teorema acima. Primeiramente, tomaremos o artigo [6] para ver sob que casos um produto em $\beta \mathbb{N}$ pode ser escrito como uma soma. 
Primeiramente, enunciamos aqui um lema sobre cancelamento. Note que como $\mathbb{N}$ é o centro de $(\beta \mathbb{N},+)$ e de $(\beta \mathbb{N}, \cdot)$, então os cancelamentos à direita a seguir também valem à esquerda.

Lema 2.14. Sejam $p, q \in \beta \mathbb{N}$ e $n, m \in \mathbb{N}$.

(a) Se $p+m=q+m$, então $p=q$.

(b) Se $p \cdot m=q \cdot m$, então $p=q$.

(c) Se $m+p=n+p$, então $m=n$.

(d) Se $m \cdot p=n \cdot p$, então $m=n$.

Demonstração. (a): Note que a função $r_{m}: \mathbb{N} \rightarrow \mathbb{N}$ (definida por $r_{m}(n)=n+m$ ) é injetora, e portanto sua extensão contínua a $\beta \mathbb{N}, \rho_{m}$, é injetora também. Assim segue a tese.

(b): Demonstração completamente análoga a (a).

(c): Suponha s.p.g. que $m \leq n$. Temos que $\mathbb{N}=\bigcup_{i=0}^{n}-i+(n+1) \mathbb{N}$ é uma união disjunta; tome $i \in\{0, \ldots, n\}$ tal que $-i+(n+1) \mathbb{N} \in m+p=n+p$. Então $-m-i+(n+1) \mathbb{N} \in p$ e $-n-i+(n+1) \mathbb{N} \in p, \operatorname{logo} m+i \equiv n+i(\bmod n+1)$, ou seja, $m \equiv n(\bmod n+1)$. Como $m \leq n$, então $m=n$.

(d): Suponha que $m \neq n$. Para cada $x \in \mathbb{N}$ e $b$ primo, seja $c(b, x)$ o número de fatores $b$ em $x$ (isto é, $c(b, x)$ é o maior $a \in \mathbb{N}$ tal que $\left.b^{a} \mid n\right)$. Como $n \neq m$, tome $b$ primo tal que $c(b, n) \neq c(b, m)$, e suponha s.p.g. que $c(b, n)<c(b, m)$. Defina $d=c(b, m)+1$ e tome $i \in\{0, \ldots, d-1\}$ tal que $A=\{x \in \mathbb{N}: c(b, x) \equiv i(\bmod d)\} \in p$. Temos então que $n A \in n \cdot p$ e $m A \in m \cdot p$. Como $n \cdot p=m \cdot p$, então podemos tomar $y \in n A \cap m A$. Portanto vale que $c(b, y) \equiv i+c(b, n)$ $(\bmod d)$, e $c(b, y) \equiv i+c(b, m)(\bmod d) ; \log c(b, n) \equiv c(b, m)(\bmod d)$ e $c(b, n)<c(b, m)<d$. Contradição.

Teorema 2.15. Sejam $p, r \in \mathbb{N}^{*}, t, s \in \beta \mathbb{N}$, e $m, n \in \mathbb{N}$. Se $t+p=m \cdot r$ e $s+p=n \cdot r$, então $m=n$.

Demonstração. Suponha, s.p.g., que $m<n$. Seja $\gamma=\log _{n} m$, e note que $0 \leq \gamma<1$, de modo que podemos tomar $d \in \mathbb{N}$ tal que $\gamma<1-\frac{2}{d}$. Note que $d \geq 3$. Para cada $i \in \mathbb{Z}$, definimos $A_{i}=\left\{x \in \mathbb{N}:\left\lfloor d \cdot \log _{n} x\right\rfloor \equiv i(\bmod 2 d)\right\}$. Observe que $A_{i}=A_{j}$ se e somente se $i \equiv j(\bmod 2 d)$, e $A_{i} \cap A_{j}=\varnothing$ caso contrário. Além disso, caso $i \in\{0, \ldots, 2 d-1\}$, temos a seguinte equivalência: $x \in A_{i} \Leftrightarrow\left\lfloor d \cdot \log _{n} x\right\rfloor \equiv i(\bmod 2 d) \Leftrightarrow \exists k \in \mathbb{N}$ tal que $n^{2 k+\frac{i}{d}} \leq x<n^{2 k+\frac{i+1}{d}}$, de forma que $A_{i}=\bigcup_{k \geq 0}\left\{x \in \mathbb{N}: n^{2 k+\frac{i}{d}} \leq x<n^{2 k+\frac{i+1}{d}}\right\}$.

Será necessário usar o seguinte fato: dados $i \in \mathbb{Z}$ e $z \in \mathbb{N},\left(-z+A_{i}\right) \backslash\left(A_{i} \cup A_{i-1}\right)$ é finito. Demonstraremos isso agora: primeiramente tome $k \in \mathbb{N}$ tal que $\frac{z}{n^{\frac{1}{d}}-1} \leq n^{2 k+\frac{i-1}{d}}$, e defina $u=n^{2 k+\frac{i}{d}}$. Mostraremos então que, se $y \in\left(-z+A_{i}\right)$ e $y \geq u$, então $y \in\left(A_{i} \cup A_{i-1}\right)$. Tome $x \in A_{i}$ 
tal que $z+y=x$, e observe que, como $x \geq y \geq u$, então $n^{2 v+\frac{i}{d}} \leq x<n^{2 v+\frac{i+1}{d}}$ para algum $v \geq k$. Como $z \leq n^{2 k+\frac{i-1}{d}} \cdot\left(n^{\frac{1}{d}}-1\right) \leq n^{2 v+\frac{i-1}{d}} \cdot\left(n^{\frac{1}{d}}-1\right)=n^{2 v+\frac{i}{d}}-n^{2 v+\frac{i-1}{d}}$, então $n^{2 v+\frac{i-1}{d}} \leq x-z=y<x<n^{2 v+\frac{i+1}{d}}$. Portanto, $2 d v+i-1 \leq d \cdot \log _{n} y<2 d v+i+1$, e assim $y \in A_{i} \cup A_{i-1}$.

Agora, vale que $\mathbb{N}=\bigcup_{i=0}^{2 d-1} A_{i}$, logo tome $i \in\{0, \ldots, 2 d-1\}$ tal que $A_{i} \in r$. Então $n A_{i} \in n \cdot r=s+p$, $\log \left\{\left\{x \in \mathbb{N}:-x+n A_{i} \in p\right\} \in s\right.$, logo tome $z \in \mathbb{N}$ tal que $-z+n A_{i} \in p$. Similarmente, $m A_{i} \in m \cdot p=t+p$, logo tome $v \in \mathbb{N}$ tal que $-v+m A_{i} \in p$. Vale que $n A_{i} \subset A_{i+d}$ (pois se $n^{2 k+\frac{i}{d}} \leq x<n^{2 k+\frac{i+1}{d}}$, então $\left.n^{2 k+\frac{i+d}{d}} \leq n \cdot x<n^{2 k+\frac{i+d+1}{d}}\right)$, $\operatorname{logo}-z+A_{i+d} \in p$. Como visto acima, $\left(-z+A_{i+d}\right) \backslash\left(A_{i+d} \cup A_{i+d-1}\right)$ é finito. Como $\left(-z+A_{i+d}\right) \backslash\left(A_{i+d} \cup A_{i+d-1}\right)=\left(-z+A_{i+d}\right) \cap$ $\left(\mathbb{N} \backslash A_{i+d}\right) \cap\left(\mathbb{N} \backslash A_{i+d-1}\right)$ e $-z+A_{i+d} \in p$, então podemos tomar $j \in\{i+d-1, i+d\}$ tal que $A_{j} \in p$. Veremos agora que $m A_{i} \subset \bigcup_{u=i}^{i+d-2} A_{u}$. Seja $x \in A_{i}$ e tome $k \geq 0$ tal que $n^{2 k+\frac{i}{d}} \leq x<n^{2 k+\frac{i+1}{d}}$. Temos então: $n^{2 k+\frac{i}{d}} \leq x \leq m \cdot x=n^{\gamma} \cdot x \leq n^{2 k+\frac{i+1}{d}+\gamma}<n^{2 k+\frac{i+1}{d}+1-\frac{2}{d}}=n^{2 k+\frac{i+d-2+1}{d}}$, do que segue a afirmação.

Desse modo, $-v+m A_{i} \subset \bigcup_{u=i}^{i+d-2}-v+A_{u}$ e $-v+m A_{i} \in p$, logo tome $u \in\{i, \ldots, i+d-2\}$ tal que $-v+A_{u} \in p$. Similarmente a acima, como $\left(-v+A_{u}\right) \backslash\left(A_{u} \cup A_{u-1}\right)$ é finito, então $A_{u} \in p$ ou $A_{u-1} \in p$. Portanto existe $w \in\{i-1, \ldots, i+d-2\}$ tal que $A_{w} \in p$. Destarte, $A_{w} \cap A_{j} \neq \varnothing$, e $\operatorname{assim} j \equiv w(\bmod 2 d)$. Porém, como $j \in\{i+d-1, i+d\}$ e $w \in\{i-1, \ldots, i+d-2\}$, isso implicaria que existem $a, b \in\{0, \ldots, d+1\}$ distintos tais que $a \equiv b(\bmod 2 d)$. Como $d>2$, isso é uma contradição.

Teorema 2.16. Sejam $p, r \in \beta \mathbb{N}$ e $m \in \mathbb{N}$. Então existe $s \in \beta \mathbb{N}$ tal que $m+p=s \cdot r$ se e somente se para cada $A \in p$ e cada função $f: \mathbb{N} \rightarrow r$ existe $n \in \mathbb{N}$ tal que $(m+A) \cap(n \cdot f(n)) \neq \varnothing$.

Demonstração. $(\Rightarrow)$ : Sejam $A \in p$ e $f: \mathbb{N} \rightarrow r$. Então $m+A \in m+p=s \cdot r$, logo

$\left\{n \in \mathbb{N}: n^{-1}(m+A) \in r\right\} \in s ;$ tome $n \in \mathbb{N}$ tal que $n^{-1}(m+A) \in r$. Então $n^{-1}(m+A) \cap f(n) \in r$, e $\operatorname{assim} \varnothing \neq(m+A) \cap(n \cdot f(n))$.

$(\Leftarrow)$ : Para cada $A \in p$ e cada $f: \mathbb{N} \rightarrow r$, defina $C(A, f)=\{n \in \mathbb{N}:(m+A) \cap(n \cdot f(n)) \neq \varnothing\}$. Por hipótese, $C(A, f) \neq \varnothing$. Afirmação: $\{C(A, f): A \in p$ e $f: \mathbb{N} \rightarrow r\}$ possui a p.i.f. Para ver isso, sejam $A_{1}, \ldots, A_{k} \in p$ e $f_{1}, \ldots, f_{k}: \mathbb{N} \rightarrow r$. Defina $A=\bigcap_{i=1}^{k} A_{i}$ e $f: \mathbb{N} \rightarrow r$ por $f(n)=\bigcap_{i=1}^{k} f_{i}(n)$. Note que, para cada $j \in\{1, \ldots, k\}$, se $x \in C(A, f)$, então $\varnothing \neq(m+A) \cap(x \cdot f(x))=(m+$ $\left.\bigcap_{i=1}^{k} A_{i}\right) \cap\left(x \cdot \bigcap_{i=1}^{k} f_{i}(x)\right) \subset\left(\bigcap_{i=1}^{k} m+A_{i}\right) \cap\left(\bigcap_{i=1}^{k} x \cdot f_{i}(x)\right) \subset\left(m+A_{j}\right) \cap\left(x \cdot f_{j}(x)\right)$, de forma que $x \in C\left(A_{j}, f_{j}\right)$. Ou seja $\varnothing \neq C(A, f) \subset \bigcap_{i=1}^{k} C\left(A_{i}, f_{i}\right)$, e assim vale a afirmação.

Desse modo, tome $s \in \beta \mathbb{N}$ tal que $\{C(A, f): A \in p$ e $f: \mathbb{N} \rightarrow r\} \subset s$. Afirmação: $m+p=s \cdot r$. Suponha que não. Tome então $A \in(m+p) \backslash(s \cdot r)$. Temos que $-m+A \in p$ e $\mathbb{N} \backslash A \in s \cdot r, \operatorname{logo}$ $B=\left\{n \in \mathbb{N}: n^{-1}(\mathbb{N} \backslash A) \in r\right\} \in s$. Defina então $f: \mathbb{N} \rightarrow r$ por $f(n)=n^{-1}(\mathbb{N} \backslash A)$ se $n \in B$ e $f(n)=\mathbb{N}$ caso contrário. Vale que $C(-m+A, f) \in s, \operatorname{logo} B \cap C(-m+A, f) \neq \varnothing$; tome $n$ nessa 
interseção. Temos então que $\varnothing \neq(m+(-m+A)) \cap(n \cdot f(n)) \subset A \cap\left(n \cdot\left(n^{-1}(\mathbb{N} \backslash A)\right)\right) \subset A \cap(\mathbb{N} \backslash A)$, uma contradição.

Teorema 2.17. Sejam $p, r \in \beta \mathbb{N}$ e $n \in \mathbb{N}$. Então existe $q \in \beta \mathbb{N}$ tal que $q+p=n \cdot r$ se e somente se para cada $A \in r$ e cada $f: \mathbb{N} \rightarrow p$ existe $m \in \mathbb{N}$ tal que $(m+f(m)) \cap(n A) \neq \varnothing$.

Demonstração. $(\Rightarrow)$ : Sejam $A \in r$ e $f: \mathbb{N} \rightarrow p$. Então $n A \in n \cdot r=q+p$, ou seja, $\{m \in \mathbb{N}$ : $-m+n A \in p\} \in q$, logo tome $m$ tal que $-m+n A \in p$. Logo $f(m) \cap(-m+n A) \in p$, e assim $\varnothing \neq(m+f(m)) \cap n A$.

$(\Leftarrow)$ : Para cada $A \in r$ e $f: \mathbb{N} \rightarrow p$, defina $C(A, f)=\{m \in \mathbb{N}: n A \cap(m+f(m)) \neq \varnothing\}$. Por hipótese, $C(A, f) \neq \varnothing$. Afirmação: $\{C(A, f): A \in r$ e $f: \mathbb{N} \rightarrow p\}$ possui a p.i.f. Para ver isso, sejam $A_{1}, \ldots, A_{k} \in r$ e $f_{1}, \ldots, f_{k}: \mathbb{N} \rightarrow p$. Defina $A=\bigcap_{i=1}^{k} A_{i}$ e $f: \mathbb{N} \rightarrow p$ por $f(m)=\bigcap_{i=1}^{k} f_{i}(m)$. Note que, para cada $j \in\{1, \ldots, k\}$, se $x \in C(A, f)$, então $\varnothing \neq(m+f(m)) \cap(x A)=\left(m+\bigcap_{i=1}^{k} f_{i}(m)\right) \cap$ $\left(x \cdot \bigcap_{i=1}^{k} A_{i}\right) \subset\left(\bigcap_{i=1}^{k}\left(m+f_{i}(m)\right)\right) \cap\left(\bigcap_{i=1}^{k} x A_{i}\right) \subset\left(m+f_{j}(m)\right) \cap\left(x A_{j}\right)$, de forma que $x \in C\left(A_{j}, f_{j}\right)$. Ou seja $\varnothing \neq C(A, f) \subset \bigcap_{i=1}^{k} C\left(A_{i}, f_{i}\right)$, e assim vale a afirmação.

Desse modo, tome $q \in \beta \mathbb{N}$ tal que $\{C(A, f): A \in r$ e $f: \mathbb{N} \rightarrow p\} \subset q$. Afirmação: $q+p=n \cdot r$. Suponha que não. Tome então $A \in(n \cdot r) \backslash(q+p)$. Temos que $n^{-1} A \in r$ e $\mathbb{N} \backslash A \in q+p$, logo $B=\{m \in \mathbb{N}:-m+\mathbb{N} \backslash A \in p\} \in q$. Defina então $f: \mathbb{N} \rightarrow p$ por $f(m)=-m+\mathbb{N} \backslash A$ se $m \in B$ e $f(m)=\mathbb{N}$ caso contrário. Vale que $C\left(n^{-1} A, f\right) \in q$, logo $B \cap C\left(n^{-1} A, f\right) \neq \varnothing$; tome $m$ nessa interseção. Temos então que $\varnothing \neq\left(n \cdot\left(n^{-1} A\right)\right) \cap(m+f(m)) \subset A \cap(m+(-m+\mathbb{N} \backslash A)) \subset A \cap(\mathbb{N} \backslash A)$, uma contradição.

Lema 2.18. Sejam $p, q, r, s \in \beta \mathbb{N}$. Então $q+p \neq s \cdot r$ se e somente se existem $B \in q, C \in s$, $f: B \rightarrow p$ e $g: C \rightarrow r$ tais que $(m+f(m)) \cap(n \cdot g(n))=\varnothing$ para todos $m \in B$ e $n \in C$.

Demonstração. $(\Rightarrow)$ : Tome $A \in(q+p) \backslash(s \cdot r)$. Considere $B=\{x \in \mathbb{N}:-x+A \in p\} \in q$ e $C=\left\{x \in \mathbb{N}: x^{-1}(\mathbb{N} \backslash A) \in r\right\} \in s$. Defina $f: B \rightarrow p$ por $f(m)=-m+A$ e $g: C \rightarrow r$ por $g(n)=n^{-1} \mathbb{N} \backslash A$. Temos então que, dados $m \in B$ e $n \in C$, que $(m+f(m)) \cap(n \cdot g(n))=$ $(m+(-m+A)) \cap\left(n \cdot\left(n^{-1}(\mathbb{N} \backslash A)\right)\right) \subset A \cap(\mathbb{N} \backslash A)=\varnothing$.

$(\Leftarrow)$ : Mostraremos que $(q+p) \backslash(s \cdot r) \neq \varnothing$. Seja $D=\bigcup_{m \in B}(m+f(m))$. Pelo teorema 1.31(d), vale que $D \in q+p$. Afirmação: $D \notin s \cdot r$. Suponha o contrário. Considere $E=\left\{x \in \mathbb{N}: x^{-1} D \in r\right\}$. $E \in s$, logo tome $n \in E \cap C$. Como $n^{-1} D \in r$, tome $x \in n^{-1} D \cap g(n)$. Temos que $n x \in D$, $\operatorname{logo}$ tome $m \in B$ tal que $n x \in(m+f(m))$. Assim, $n x \in(m+f(m)) \cap(n \cdot g(n))$, uma contradição.

Teorema 2.19. Sejam $p, r \in \mathbb{N}^{*}, G=\left\{q \in \mathbb{N}^{*}: q+p=s \cdot r\right.$ para algum $\left.s \in \mathbb{N}^{*}\right\}$, e $H=\{m \in \mathbb{N}$ : $m+p=t \cdot r$ para algum $t \in \beta \mathbb{N}\}$. São equivalentes:

(a) $G \neq \varnothing$. 
(b) $|H|=\omega$.

(c) $|G|=2^{\mathfrak{c}}$.

Demonstração. (a) $\Rightarrow(\mathrm{b})$ : Tome $q \in G$. Tome $s \in \mathbb{N}^{*}$ tal que $q+p=s \cdot r$. Suponha por absurdo que $H$ é finito. Considere $B=\mathbb{N} \backslash H$ e $C=\{n \in \mathbb{N}$ : para todo $t \in \beta \mathbb{N}, t+p \neq n \cdot r\}$. Note que $|\mathbb{N} \backslash C| \leq 1$, pois se $m, n \in \mathbb{N} \backslash C$, então existem $t, s \in \beta \mathbb{N}$ tais que $t+p=m \cdot r$ e $s+p=n \cdot r$; pelo teorema $2.15, m=n$. Assim, $B \in q$ e $C \in s$.

Observe que $B=\mathbb{N} \backslash H=\{m \in \mathbb{N}$ : não existe $t \in \beta \mathbb{N}$ tal que $m+p=t \cdot r\}$. Portanto, para cada $m \in B$, existem pelo teorema $2.16 A_{m} \in p$ e $g_{m}: \mathbb{N} \rightarrow r$ tais que $\left(m+A_{m}\right) \cap\left(n \cdot g_{m}(n)\right)=\varnothing$ para todo $n \in \mathbb{N}$. Similarmente, como $C=\{n \in \mathbb{N}$ : não existe $t \in \beta \mathbb{N}$ tal que $t+p=n \cdot r\}$, pelo teorema 2.17, para cada $n \in C$ existem $D_{n} \in r$ e $f_{n}: \mathbb{N} \rightarrow p$ tais que, para todo $m \in \mathbb{N}$, $\left(m+f_{n}(m)\right) \cap\left(n D_{n}\right)=\varnothing$.

Defina então $f: B \rightarrow p$ por $f(m)=A_{m} \cap\left(\bigcap\left\{f_{n}(m): n \in C\right.\right.$ e $\left.\left.n \leq m\right\}\right)$, e $g: C \rightarrow r$ por $g(n)=D_{n} \cap\left(\bigcap\left\{g_{m}(n): m \in B\right.\right.$ e $\left.\left.m \leq n\right\}\right)$. Assim, pelo lema 2.18, existem $m \in B$ e $n \in C$ tais que $(m+f(m)) \cap(n \cdot g(n)) \neq \varnothing$. Porém, se $n \leq m, f(m) \subset f_{n}(m)$ e $g(n) \subset D_{n}$, de forma que $\left(m+f_{n}(m)\right) \cap\left(n D_{n}\right) \neq \varnothing$, uma contradição; e se $m \leq n, f(m) \subset A_{m}$ e $g(n) \subset g_{m}(n)$, de forma que $\left(m+A_{m}\right) \cap\left(n \cdot g_{m}(n)\right) \neq \varnothing$, outra contradição.

(b) $\Rightarrow$ (c): É uma consequência mais direta da topologia de $\beta \mathbb{N}$. Primeiramente, notamos que dado $X \subset \beta \mathbb{N}, \mathbb{N} \cap(\operatorname{cl} X)=\mathbb{N} \cap X$, pois $\mathbb{N}$ é o conjunto dos pontos isolados de $\beta \mathbb{N}$. Lembramos que, pelo teorema 1.26(d), $\left|H^{*}\right|=2^{\mathfrak{c}}$. Logo, basta ver que dado $q \in H^{*}$, existe $s \in \mathbb{N}^{*}$ tal que $q+p=s \cdot r$.

Seja $q \in H^{*}$. Para cada $B \in q$, defina $C(B)=\{t \in \beta \mathbb{N}: m+p=t \cdot r$ para algum $m \in B\}$. Note que, como $H \cap B \neq \varnothing, C(B) \neq \varnothing$; além disso, dados $B_{1}, B_{2} \in q, C\left(B_{1} \cap B_{2}\right) \subset C\left(B_{1}\right) \cap C\left(B_{2}\right)$. Destarte, $\mathcal{A}=\{\operatorname{cl} C(B): B \in q\}$ é uma coleção de fechados de $\beta \mathbb{N}$ com a p.i.f., $\operatorname{logo} \bigcap \mathcal{A} \neq \varnothing$. Tome $s \in \bigcap \mathcal{A}$.

Primeiro é preciso ver que $s \in \mathbb{N}^{*}$. Suponha $s \in \mathbb{N}$. Então $s \in \mathbb{N} \cap\left(\bigcap_{B \in q} \operatorname{cl} C(B)\right)=$ $=\bigcap_{B \in q} \mathbb{N} \cap(\operatorname{cl} C(B))=\bigcap_{B \in q} \mathbb{N} \cap C(B) \subset \bigcap_{B \in q} C(B)$. Em particular, $s \in C(H)$, logo tome $m \in H$ tal que $m+p=s \cdot r$. Vale também que $H \backslash\{m\} \in q$, logo $s \in C(H \backslash\{m\})$; tome $n \in H \backslash\{m\}$ tal que $n+p=s \cdot r$. Então $m+p=n+p$, uma contradição, pelo lema 2.14.

Por fim, veremos que $q+p=s \cdot r$. Suponha que $q+p \neq s \cdot r$. Pelo lema 2.18, tome $B \in q, C \in s$, $f: B \rightarrow p$ e $g: C \rightarrow r$ tais que $(m+f(m)) \cap(n \cdot g(n))=\varnothing$ para todos $m \in B$ e $n \in C$. Como $\bar{C}$ é uma vizinhança de $s$ e $s \in \operatorname{cl} C(B)$, tome $t \in \bar{C} \cap C(B)$; podemos então tomar $m \in B$ tal que $m+p=t \cdot r$. Porém, $m \in B$ implica que $B \in m$ (lembrando a identificação de $m$ com seu ultrafiltro principal), e $C \in t$. Logo, pelo lema $2.18, m+p \neq t \cdot r$.

Agora podemos provar o teorema citado na demonstração de 2.13 .

Teorema 2.20. Seja $r \in \mathbb{N}^{*}$. Se $|\{n \in \mathbb{N}: n \mathbb{N} \in r\}|=\omega$, então para todos $p, q, s \in \mathbb{N}^{*}, s \cdot r \neq q+p$. 
Demonstração. Suponha que $s \cdot r=q+p$. Pelo teorema 2.19, temos que $\mid\{m \in \mathbb{N}: m+p=t \cdot r$ para algum $t \in \beta \mathbb{N}\} \mid=\omega$. Logo tome $m, n \in \mathbb{N}, m<n$, e $t_{0}, t_{1} \in \beta \mathbb{N}$ tais que $m+p=t_{0} \cdot r$ e $n+p=t_{1} \cdot r$. Tome $a>n$ tal que $a \mathbb{N} \in r$. Como $\mathbb{N}=\bigcup_{i=0}^{a-1} i+a \mathbb{N}$, tome $i$ tal que $i+a \mathbb{N} \in p$. Então $m+i+a \mathbb{N} \in m+p=t_{0} \cdot r, \operatorname{logo}\left\{x \in \mathbb{N}: x^{-1}(m+i+a \mathbb{N}) \in r\right\} \in t_{0}$, logo tome $x \in \mathbb{N}$ tal que $x^{-1}(m+i+a \mathbb{N}) \in r$. Analogamente, tome $y \in \mathbb{N}$ tal que $y^{-1}(n+i+a \mathbb{N}) \in r$. Tome então $z \in a \mathbb{N} \cap x^{-1}(m+i+a \mathbb{N}) \cap y^{-1}(n+i+a \mathbb{N})$. Temos então que $x z \in a \mathbb{N} \cap(m+i+a \mathbb{N})$, portanto $m+i \equiv 0(\bmod a) ;$ e $y z \in a \mathbb{N} \cap(n+i+a \mathbb{N})$, portanto $n+i \equiv 0(\bmod a)$. Deste modo, $m \equiv n$ $(\bmod a) \operatorname{com} m<n<a$, uma contradição.

Para concluir este trecho, e ver que o teorema acima se aplica ao teorema 2.13, basta o seguinte lema:

Lema 2.21. Seja $q \in \operatorname{cl}\{p \in \beta \mathbb{N}: p+p=p\}$. Então para todo $n \in \mathbb{N}, n \mathbb{N} \in q$.

Demonstração. Queremos ver que $n \mathbb{N} \in q$, isto é, $q \in \overline{n \mathbb{N}}$. Seja $\bar{A}$ uma vizinhança básica de $q$, isto é, seja $A \in q$. Vale que $\bar{A} \cap\{p \in \beta \mathbb{N}: p+p=p\} \neq \varnothing$, logo tome $p=p+p$ tal que $A \in p$. Pelo lema $2.2, n \mathbb{N} \in p$, e portanto $A \cap n \mathbb{N} \neq \varnothing$.

Agora, partimos para ver que o conjunto $M$ do teorema 2.13 é um ideal à esquerda de $(\beta \mathbb{N}, \cdot)$. Para tanto, precisaremos de uma caracterização de ideais à esquerda minimais, que pode ser obtida estudando a estrutura dos ultrafiltros $\kappa$-uniformes. A próxima definição e os próximos lemas e teoremas são, respectivamente, a Definição 2.3, o Lema 2.4, o Teorema 2.5, o Lema 3.1 e o Teorema 3.2 de [4].

Definição 2.22. Seja $(S, \cdot)$ um semigrupo discreto. Sejam $p \in \beta S, \kappa=1$ ou $\kappa \geq \omega$. Então $C_{\kappa}(p)=\left\{A \subset S:\left|\left\{x \in S: x^{-1} A \notin p\right\}\right|<\kappa\right\}$.

Note que $C_{\kappa}(p)=\left\{A \subset S:\left|\left\{x \in S: x^{-1}(S \backslash A) \in p\right\}\right|<\kappa\right\}$. Portanto, $S \backslash A \in C_{\kappa}(p) \Leftrightarrow \mid\{x \in$ $\left.S: x^{-1} A \in p\right\} \mid<\kappa$. Em particular, quando $\kappa=1, C_{1}(p)=\left\{A \subset S:\right.$ para todo $\left.x \in S, x^{-1} A \in p\right\}$.

Lema 2.23. Sejam $p \in \beta S$ e $\kappa \leq|S| \operatorname{com} \kappa=1$ ou $\kappa \geq \omega$.

(a) $C_{\kappa}(p)$ é um filtro em $S$.

(b) $\mathcal{U}_{\kappa}(S) \cdot p=\widehat{C_{\kappa}(p)}=\left\{q \in \beta S: C_{\kappa}(p) \subset q\right\}$.

Demonstração. (a): $S \in C_{\kappa}(p)$ pois $\left|\left\{x \in S: x^{-1}(S \backslash S) \in p\right\}\right|=|\varnothing|<\kappa . \quad \varnothing \notin C_{\kappa}(p)$ pois $\left|\left\{x \in S: x^{-1}(S \backslash \varnothing) \in p\right\}\right|=|S| \geq \kappa$. Dado $A \in C_{\kappa}(p)$ e $B \supset A$, vale que $x^{-1}(S \backslash B) \subset x^{-1}(S \backslash A)$, $\log \left\{x \in S: x^{-1}(S \backslash B) \in p\right\} \subset\left\{x \in S: x^{-1}(S \backslash A) \in p\right\}$, e assim $B \in C_{\kappa}(p)$. Por fim, dados $A, B \in C_{\kappa}(p)$, tem-se que $\left\{x \in S: x^{-1}(S \backslash(A \cap B)) \in p\right\}=\left\{x \in S: x^{-1}(S \backslash A) \cup x^{-1}(S \backslash B) \in p\right\}=$ $\left\{x \in S: x^{-1}(S \backslash A) \in p\right\} \cup\left\{x \in S: x^{-1}(S \backslash B) \in p\right\}, \log 0 A \cap B \in C_{\kappa}(p)$. 
(b): Primeiramente veremos que $\mathcal{U}_{\kappa}(S) \cdot p \subset \widehat{C_{\kappa}(p)}$. Seja $r \in \mathcal{U}_{\kappa}(S)$, e seja $A \in C_{\kappa}(p)$. $\mid\{x \in S$ : $\left.x^{-1} A \notin p\right\} \mid<\kappa, \log 0 S \backslash\left\{x \in S: x^{-1} A \notin p\right\}=\left\{x \in S: x^{-1} A \in p\right\} \in r$. Ou seja, $A \in r \cdot p$. Portanto $C_{\kappa}(p) \subset r \cdot p$.

Agora veremos que $\widehat{C_{\kappa}(p)} \subset \mathcal{U}_{\kappa}(S) \cdot p$. Logo seja $q \in \beta S$ tal que $C_{\kappa}(p) \subset q$. Defina, para cada $A \in q, T(A)=\left\{x \in S: x^{-1} A \in p\right\}$. Note que dados $A, B \in q, T(A \cap B)=T(A) \cap T(B)$; e que se $A \in q, S \backslash A \notin C_{\kappa}(p)$ (pois $C_{\kappa}(p) \subset q$ ), e portanto $|T(A)| \geq \kappa$. Deste modo, $\{T(A): A \in q\}$ possui a $\kappa$-p.i.f., logo tome $r \in \mathcal{U}_{\kappa}(S)$ tal que $\{T(A): A \in q\} \subset r$. Então, dado $A \in q, T(A)=\{x \in S$ : $\left.x^{-1} A \in p\right\} \in r$, ou seja, $A \in r \cdot p$. Portanto, $q \subset r \cdot p$, e assim $q=r \cdot p$.

Não é surpreendente que $\mathcal{U}_{\kappa}(S) \cdot p=\widehat{\mathcal{F}}$ para um filtro $\mathcal{F}$, pois sabemos que é um fechado, já que $\mathcal{U}_{\kappa}(S) \cdot p=\rho_{p}\left[\mathcal{U}_{\kappa}(S)\right]$. O que o lema acima faz é dar a este filtro uma forma que seja boa de trabalhar.

Além disso, sabemos que $\mathcal{U}_{\kappa}(S)$ também é fechado, pois se $p \notin \mathcal{U}_{\kappa}(S)$, dado $A \in p \operatorname{com}|A|<\kappa$, $\bar{A}$ é uma vizinhança de $p$ disjunta de $\mathcal{U}_{\kappa}(S)$. Veremos agora uma condição necessária e suficiente sobre a álgebra de $S$ para que $\mathcal{U}_{\kappa}(S)$ seja um subsemigrupo.

Note que no enunciado abaixo um hipotético caso $\kappa=1$ seria trivialmente satisfeito.

Teorema 2.24. Seja $\omega \leq \kappa \leq|S|$. São equivalentes:

(a) $\mathcal{U}_{\kappa}(S)$ é um subsemigrupo de $\beta S$.

(b) Para todo $p \in \mathcal{U}_{\kappa}(S)$ e todo $A \in[S]^{<\kappa}, S \backslash A \in C_{\kappa}(p)$.

(c) Para todo $A \in[S]^{<\kappa}$ e todo $B \in[S]^{\kappa}$, existe $F \in \mathcal{P}_{f}(B)$ tal que $\left|\bigcap_{x \in F} x^{-1} A\right|<\kappa$.

Demonstração. (a) $\Rightarrow(\mathrm{b})$ : Por contraposição. Tome $p \in \mathcal{U}_{\kappa}(S)$ e $A \in[S]^{<\kappa}$ tais que $S \backslash A \notin C_{\kappa}(p)$. Então $C_{\kappa}(p) \cup\{A\}$ possui a p.i.f. (pois se $B \in C_{\kappa}(p)$ e $B \cap A=\varnothing$, então $B \subset S \backslash A$, $\operatorname{logo}$ $\left.S \backslash A \in C_{\kappa}(p)\right)$. Logo tome $q \in \beta S$ tal que $C_{\kappa}(p) \cup\{A\} \subset q$. Pelo lema 2.23(b), existe $r \in \mathcal{U}_{\kappa}(S)$ tal que $q=r \cdot p$. Como $A \in q, q \notin \mathcal{U}_{\kappa}(S)$, ou seja, $r \cdot p \notin \mathcal{U}_{\kappa}(S)$, e portanto $\mathcal{U}_{\kappa}(S)$ não é subsemigrupo. (b) $\Rightarrow(\mathrm{c})$ : Por contraposição. Tome $A \in[S]^{<\kappa}$ e $B \in[S]^{\kappa}$ tais que, para todo $F \in \mathcal{P}_{f}(B)$, $\left|\bigcap_{x \in F} x^{-1} A\right| \geq \kappa$. Então $\left\{x^{-1} A: x \in B\right\}$ possui a $\kappa$-p.i.f., logo tome $p \in \mathcal{U}_{\kappa}(S)$ tal que $\left\{x^{-1} A: x \in B\right\} \subset p$. Então $B \subset\left\{x \in S: x^{-1} A \in p\right\}$. Como $|B|=\kappa$, temos que $S \backslash A \notin C_{\kappa}(p)$, como pretendíamos.

(c) $\Rightarrow\left(\right.$ a): Por contraposição. Tome $r, p \in \mathcal{U}_{\kappa}(S)$ tais que $r \cdot p \notin \mathcal{U}_{\kappa}(S)$. Seja $q=r \cdot p$. $q \notin \mathcal{U}_{\kappa}(S)$, logo tome $A \in q$ tal que $|A|<\kappa$. Considere $D=\left\{x \in S: x^{-1} A \in p\right\}$. Vale que $D \in r$, $\operatorname{logo}$ $|D| \geq \kappa$, e portanto podemos tomar $B \in[D]^{\kappa}$. Deste modo, para todo $F \in \mathcal{P}_{f}(B), \bigcap_{x \in F} x^{-1} A \in p$, $\log 0\left|\bigcap_{x \in F} x^{-1} A\right| \geq \kappa$.

Observe que, se $S$ for cancelativo à esquerda, então $\mathcal{U}_{\kappa}(S)$ é um subsemigrupo. 
Agora, iremos provar uma condição necessária e suficiente para que $\mathcal{U}_{\kappa}(S) \cdot p$ seja um ideal à esquerda minimal de $\beta S$.

Lema 2.25. Seja $\kappa \leq|S|$ tal que $\kappa=1$ ou $\kappa \geq \omega$. Se $\mathcal{U}_{\kappa}(S)$ é um subsemigrupo de $\beta S$, dados $A \subset S, p \in \mathcal{U}_{\kappa}(S)$, se $S \backslash A \notin C_{\kappa}(p)$, então $C_{\kappa}(p) \cup\{A\}$ tem a $\kappa$-p.i.f.

Demonstração. Pelo lema 2.23(a), $C_{\kappa}(p)$ é filtro, logo basta ver que dado $D \in C_{\kappa}(p),|D \cap A| \geq \kappa$. Suponha que $|D \cap A|<\kappa$. Como $S \backslash A \notin C_{\kappa}(p),\left|\left\{x \in S: x^{-1} A \in p\right\}\right| \geq \kappa$. Como $D \in C_{\kappa}(p)$, $\left|\left\{x \in S: x^{-1} D \notin p\right\}\right|<\kappa$. Portanto, $\mid\left\{x \in S: x^{-1} A \in p\right.$ e $\left.x^{-1} D \in p\right\}|=|\left\{x \in S: x^{-1}(A \cap D) \in\right.$ $p\} \mid \geq \kappa$. Tome então $B \in\left[\left\{x \in S: x^{-1}(A \cap D) \in p\right\}\right]^{\kappa}$. Como $A \cap D \in[S]^{<\kappa}, B \in[S]^{\kappa}$, e $\mathcal{U}_{\kappa}(S)$ é subsemigrupo, então seja $F \in \mathcal{P}_{f}(B)$ tal que $\left|\bigcap_{x \in F} x^{-1}(A \cap D)\right|<\kappa$. Porém, $\bigcap_{x \in F} x^{-1}(A \cap D) \in p$ e $p \in \mathcal{U}_{\kappa}(S)$. Contradição.

Teorema 2.26. Seja $\kappa \leq|S|$ tal que $\kappa=1$ ou $\kappa \geq \omega$, suponha que $\mathcal{U}_{\kappa}(S)$ é um subsemigrupo de $\beta S$ e seja $p \in \mathcal{U}_{\kappa}(S)$. Então $\mathcal{U}_{\kappa}(S) \cdot p$ é um ideal à esquerda minimal de $\mathcal{U}_{\kappa}(S)$ se e somente se dados $B \in[S]^{<\kappa}$ e $A \subset S$ tal que $S \backslash A \notin C_{\kappa}(p)$, existe $F \in \mathcal{P}_{f}(S \backslash B)$ tal que $\bigcup_{x \in F} x^{-1} A \in C_{\kappa}(p)$.

Demonstração. $(\Rightarrow)$ : Sejam $B \in[S]^{<\kappa}$ e $A \subset S$ tal que $S \backslash A \notin C_{\kappa}(p)$. Pelo lema anterior, $C_{\kappa}(p) \cup\{A\}$ possui a $\kappa$-p.i.f., logo tome $r \in \mathcal{U}_{\kappa}(p)$ tal que $C_{\kappa}(p) \cup\{A\} \subset r$.

Suponha que para cada $F \in \mathcal{P}_{f}(S \backslash B), \bigcup_{x \in F} x^{-1} A \notin C_{\kappa}(p)$. Afirmamos que $C_{\kappa}(p) \cup\left\{S \backslash\left(\bigcup_{x \in F} x^{-1} A\right)\right.$ : $\left.F \in \mathcal{P}_{f}(S \backslash B)\right\}$ possui a $\kappa$-p.i.f. Observe que $\left\{S \backslash\left(\bigcup_{x \in F} x^{-1} A\right): F \in \mathcal{P}_{f}(S \backslash B)\right\}$ é fechado para interseção, pois dados $F, G \in \mathcal{P}_{f}(S \backslash B),\left(S \backslash\left(\bigcup_{x \in F} x^{-1} A\right)\right) \cap\left(S \backslash\left(\bigcup_{x \in G} x^{-1} A\right)\right)=S \backslash\left(\bigcup_{x \in F \cup G} x^{-1} A\right)$. Portanto, basta ver que dado $F \in \mathcal{P}_{f}(S \backslash B), C_{\kappa}(p) \cup\left\{S \backslash\left(\bigcup_{x \in F \cup G} x^{-1} A\right)\right\}$ possui a $\kappa$-p.i.f. Isso segue diretamente do lema anterior, já que supusemos que $\bigcup_{x \in F \cup G} x^{-1} A \notin C_{\kappa}(p)$. Portanto, pode$\operatorname{mos}$ tomar $q \in \mathcal{U}_{\kappa}(S)$ tal que $C_{\kappa}(p) \cup\left\{S \backslash\left(\bigcup_{x \in F} x^{-1} A\right): F \in \mathcal{P}_{f}(S \backslash B)\right\} \subset q$.

Pelo lema 2.23(b), temos que $q, r \in \mathcal{U}_{\kappa}(S) \cdot p$. Como, por hipótese, $\mathcal{U}_{\kappa}(S) \cdot p$ é um ideal à esquerda minimal de $\mathcal{U}_{\kappa}(S)$, então $\mathcal{U}_{\kappa}(S) \cdot q=\mathcal{U}_{\kappa}(S) \cdot p$, e assim $r \in \mathcal{U}_{\kappa}(S) \cdot q$. Logo existe $s \in \mathcal{U}_{\kappa}(S)$ tal que $r=s \cdot q$. Como $A \in r=s \cdot q,\left\{x \in S: x^{-1} A \in q\right\} \in s, \operatorname{logo}\left|\left\{x \in S: x^{-1} A \in q\right\}\right| \geq \kappa$. Como $B \in[S]^{<\kappa}$, podemos tomar $x \in S \backslash B$ tal que $x^{-1} A \in q$. Por outro lado, $\{x\} \in \mathcal{P}_{f}(S \backslash B)$, $\operatorname{logo}$ $S \backslash\left(x^{-1} A\right) \in q$. Contradição.

$(\Leftarrow)$ : Para ver que $\mathcal{U}_{\kappa}(S) \cdot p$ é um ideal à esquerda minimal, basta ver que dados $q, r \in \mathcal{U}_{\kappa}(S) \cdot p$, existe $s \in \mathcal{U}_{\kappa}(S)$ tal que $r=s \cdot q$. Pois então dados $L \subset \mathcal{U}_{\kappa}(S) \cdot p$ ideal à esquerda, e $r \in \mathcal{U}_{\kappa}(S) \cdot p$, tome $q \in L$, e tome $s \in \mathcal{U}_{\kappa}(S)$ tal que $r=s \cdot q$. Então $r \in L$, ou seja $\mathcal{U}_{\kappa}(S) \cdot p \subset L$.

Portanto, sejam $q, r \in \mathcal{U}_{\kappa}(S) \cdot p$. Temos que $C_{\kappa}(p) \subset q, r$.

Para cada $A \in r$, defina $T(A)=\left\{x \in S: x^{-1} A \in q\right\}$. Então, similarmente à prova do lema 2.23(b), basta ver que para cada $A \in r,|T(A)| \geq \kappa$. Fixe então $A \in r$. Como $C_{\kappa}(p) \subset r$, temos que $S \backslash A \notin C_{\kappa}(p)$. Tome então (pela hipótese) $F_{0} \in \mathcal{P}_{f}(S)$ tal que $\bigcup_{x \in F_{0}} x^{-1} A \in C_{\kappa}(p)$. Agora, suponha que $\tau<\kappa$ e para todo $\sigma<\tau$ foi escolhido $F_{\sigma} \in \mathcal{P}_{f}\left(S \backslash \bigcup_{\eta<\sigma} F_{\eta}\right)$ de forma que $\bigcup_{x \in F_{\sigma}} x^{-1} A \in$ 
$C_{\kappa}(p)$. Vale que $\left|\bigcup_{\sigma<\tau} F_{\sigma}\right|<\kappa$, logo, pela hipótese podemos tomar $F_{\tau} \in \mathcal{P}_{f}\left(S \backslash \bigcup_{\sigma<\tau} F_{\sigma}\right)$ tal que $\bigcup_{x \in F_{\tau}} x^{-1} A \in C_{\kappa}(p)$. Como $C_{\kappa}(p) \subset q$, temos que para cada $\tau<\kappa, \bigcup_{x \in F_{\tau}} x^{-1} A \in q$, logo tome $x_{\tau} \in F_{\tau}$ tal que $x_{\tau}{ }^{-1} A \in q$. Desse modo, $\left\{x_{\tau}: \tau<\kappa\right\} \subset T(A)$. Como $\left\{F_{\tau}: \tau<\kappa\right\}$ é uma coleção dois-a-dois disjunta, segue que $|T(A)| \geq \kappa$, como desejado.

Agora, podemos provar como corolário o Lema 1.1 de [5].

Corolário 2.27. Sejam $(S, \cdot)$ um semigrupo discreto e $p \in \beta S$. Então $\beta S \cdot p$ é um ideal à esquerda minimal se e somente se dado $A \subset S$, se existe $z \in S$ tal que $z^{-1} A \in p$, então existe $F \in \mathcal{P}_{f}(S)$ tal que para todo $y \in S, y^{-1}\left(\bigcup_{x \in F} x^{-1} A\right) \in p$.

Demonstração. Considere o teorema anterior no caso $\kappa=1$. Então $\mathcal{U}_{1}(S)=\beta S .[S]^{<1}=\{\varnothing\}, \operatorname{logo}$ temos a seguinte asserção:

Dado $p \in \beta S, \beta S \cdot p$ é um ideal à esquerda minimal se e somente se dado $A \subset S$, se $S \backslash A \notin C_{1}(p)$, então existe $F \in \mathcal{P}_{f}(S)$ tal que $\bigcup_{x \in F} x^{-1} A \in C_{1}(p)$.

Porém, como foi observado, $C_{1}(p)=\left\{A \subset S\right.$ : para todo $\left.x \in S, x^{-1} A \in p\right\}$. Logo $S \backslash A \notin C_{1}(p)$ é equivalente a existir $z \in S$ tal que $z^{-1}(S \backslash A) \notin p$, ou seja, $z^{-1} A \in p$; e $\bigcup_{x \in F} x^{-1} A \in C_{1}(p)$ é equivalente a valer que $y^{-1}\left(\bigcup_{x \in F} x^{-1} A\right) \in p$ para todo $y \in S$. Assim, traduzindo isso à asserção acima, temos o resultado desejado.

O próximo lema foi também retirado de [5] (é o Lema 1.2), e trata de um caso especial em que vale a distributividade em $\beta \mathbb{N}$.

Lema 2.28. Sejam $p, q \in \beta \mathbb{N}$ e $x \in \mathbb{N}$. Então $x \cdot(p+q)=x \cdot p+x \cdot q$.

Demonstração. Como ambos são ultrafiltros, basta ver que $x \cdot(p+q) \subset x \cdot p+x \cdot q$. Seja $A \in x \cdot(p+q)$. Vale que $x^{-1} A \in(p+q)$, $\log 0 B=\left\{y \in \mathbb{N}:-y+x^{-1} A \in q\right\} \in p$. Portanto $x \cdot B \in x \cdot p$. Portanto, basta ver que $x \cdot B \subset\{y \in \mathbb{N}:-y+A \in x \cdot q\}$ (pois daí $A \in x \cdot p+x \cdot q$ ). Logo seja $z \in B$. Vale que $-z+x^{-1} A \in q, \log -x \cdot z+A \in x \cdot q$, como afirmado.

Agora estamos prontos para provar que aquele conjunto $M$ do 2.13 é um ideal à esquerda de $(\beta \mathbb{N}, \cdot)$. Este é o Teorema 5.4 de [5], devido a Vitaly Bergelson e Neil Hindman.

Teorema 2.29. Seja $M=\operatorname{cl}\{p \in \beta \mathbb{N}: p$ é idempotente minimal de $(\beta \mathbb{N},+)\}$. Então $M$ é um ideal à esquerda de $(\beta \mathbb{N}, \cdot)$.

Demonstração. Sejam $q \in M, r \in \beta \mathbb{N}$, e $A \in r \cdot q$. Precisamos ver que existe um idempotente minimal de $(\beta \mathbb{N},+)$ a que $A$ pertença. Sabemos que $\left\{x \in \mathbb{N}: x^{-1} A \in q\right\} \in r$, logo fixe $x \in \mathbb{N}$ tal que $x^{-1} A \in q$. Como $q \in M$, então existe $p$ idempotente minimal de $(\beta \mathbb{N},+)$ tal que $x^{-1} A \in p$. Portanto, $A \in x \cdot p$. Pelo lema 2.28 , temos que $x \cdot p+x \cdot p=x \cdot(p+p)=x \cdot p, \operatorname{logo} x \cdot p$ é idempotente. 
Assim, resta ver que $x \cdot p$ é minimal em $(\beta \mathbb{N},+)$.

Para ver que $x \cdot p$ é minimal em $(\beta \mathbb{N},+)$, basta ver, pelo teorema 1.16 , que $\beta \mathbb{N}+x \cdot p$ é um ideal à esquerda minimal de $(\beta \mathbb{N},+)$; pelo mesmo teorema, sabemos que $\beta \mathbb{N}+p$ é um ideal à esquerda minimal; logo em ambos os casos aplicaremos o corolário 2.27 .

Seja $B \subset \mathbb{N}$ tal que existe $n \in \mathbb{N}$ tal que $-n+B \in x \cdot p$. Pelo corolário 2.27, precisamos obter $F \in \mathcal{P}_{f}(\mathbb{N})$ tal que para todo $y \in \mathbb{N},-y+\left(\bigcup_{z \in F}-z+B\right) \in x \cdot p$. Tome $n \in \mathbb{N}$ tal que $-n+B \in x \cdot p$. Tome $i \in\{0, \ldots, x-1\}$ tal que $n+i \in x \mathbb{N}$. Então $-(n+i)+(i+B)=-n+B \in x \cdot p, \operatorname{logo}$ $x^{-1}(-(n+i)+(i+B))=-\left(\frac{n+i}{x}\right)+x^{-1}(i+B) \in p$. Defina $C=x^{-1}(i+B)$. Como $-\left(\frac{n+i}{x}\right)+C \in p$, tome pelo corolário $2.27 F \in \mathcal{P}_{f}(\mathbb{N})$ tal que, para todo $y \in \mathbb{N},-y+\left(\bigcup_{z \in F}-z+C\right) \in p$. Tome $k \in \mathbb{N}$ tal que $F \subset\{1, \ldots, k\}$ e defina $G=\{1, \ldots, x(k+1)\}$.

Afirmação: para todo $y \in \mathbb{N},-y+\left(\bigcup_{z \in G}-z+B\right) \in x \cdot p$. Para ver isso, seja $y \in \mathbb{N}$. Tome $a \in \mathbb{N}$ tal que $x(a-1) \leq y<x a$. Vale que $-a+\left(\bigcup_{z \in F}-z+C\right) \in p$, e $-a+\left(\bigcup_{z \in F}-z+C\right)=\bigcup_{z \in F}-(z+a)+C$; logo tome $t \in F$ tal que $-(t+a)+C \in p$. Vale então que $-x(t+a)+x C \in x \cdot p$. Lembrando que $C=x^{-1}(i+B)$, temos que $-x(t+a)+x C \subset-x(t+a)+(i+B)=-x t-x a+i+B, \operatorname{logo}$ $-x t-x a+i+B \in x \cdot p$. Seja $s \in\{1, \ldots, x\}$ tal que $x a=s+y$. Então $-x t-x a+i+B=-y+$ $(-(x t+s-i)+B)$. Agora, note que, como $i \in\{0, \ldots, x-1\}$ e $s \in\{1, \ldots, x\}, s-i \in\{-x+2, \ldots, x\}$; e como $t \in F \subset\{1, \ldots, k\}, x t \in\{x, \ldots, x k\}$; portanto $x t+s-i \in\{2, \ldots, x(k+1)\} \subset G$. Dessa forma, $-y+\left(-(x t+s-i)+B \subset-y+\left(\bigcup_{z \in G}-z+B\right)\right.$, e assim $-y+\left(\bigcup_{z \in G}-z+B\right) \in x \cdot p$, como afirmado. 


\section{$2.3 \mathbb{N}^{*}$ não contém uma cópia algébrica e topológica de $\beta \mathbb{N}$}

Esta seção é o estudo do surpreendente resultado (postulado acima) publicado por Dona Strauss em 1992, em [7], e suas consequências.

Lema 2.30. Sejam $k \in \mathbb{N}$ e $p \in \mathbb{N}^{*}$ idempotente. Então para todo $q \in \mathbb{N}^{*}$ existe $r \in \overline{k \mathbb{N}}$ tal que $r+q+p \neq q+r+p$.

Demonstração. Seja $q \in \mathbb{N}^{*}$. Podemos supor que $k>1$. No caso $k=1$, podemos usar o caso $k=2$ e tomar $r \in \overline{2 \mathbb{N}}$ tal que $r+q+p \neq q+r+p$. Valerá que $r \in \overline{\mathbb{N}}=\beta \mathbb{N}$.

Assim, suponha $k>1$, e suponha por absurdo que para todo $r \in \overline{k \mathbb{N}}, r+q+p=q+r+p$. Para cada $m \in \mathbb{N}$ considere sua expansão (única) em base $k, \sum_{n \in \omega} a_{n} k^{n}$; como $a_{n} \in\{0, \ldots, k-1\}$ para todo $n$, temos uma função $f: \mathbb{N} \rightarrow\{0, \ldots, k-1\}^{\omega}$ definida por $f(m)=\left(a_{n}\right)_{n \in \omega}$. Além disso, defina também $g: \mathbb{N} \rightarrow \mathbb{N}$ e $c: \mathbb{N} \rightarrow \mathbb{N}$ por: se $m=\sum_{n \in \omega} a_{n} k^{n}, g(m)=a_{n} k^{n}$ em que $n$ é o menor índice tal que $a_{n} \neq 0$, e $c(m)=\left|\left\{n \in \omega: a_{n} \neq 0\right\}\right|$. Lembre que $f, g$ e $c$ possuem extensões contínuas $\tilde{f}: \beta \mathbb{N} \rightarrow\{0, \ldots, k-1\}^{\omega}$ e $g, c: \beta \mathbb{N} \rightarrow \beta \mathbb{N}$.

Vejamos agora que, dados $s \in \beta \mathbb{N}$ e $t \in \bigcap_{n \in \mathbb{N}} \overline{k^{n} \mathbb{N}}$, vale que $\widetilde{g}(s+t)=\widetilde{g}(s)$, e $\widetilde{c}(s+t)=\widetilde{c}(s)+\widetilde{c}(t)$. Para a primeira igualdade, basta ver que as funções $\widetilde{g} \circ \rho_{t}$ e $\widetilde{g}$ coincidem sobre $\mathbb{N}$; para a segunda, basta ver que as funções $\widetilde{c} \circ \rho_{t}$ e $\rho_{\widetilde{c}(t)} \circ \widetilde{c}$ coincidem sobre $\mathbb{N}$. Seja $m \in \mathbb{N}$ então. Quero ver que $\widetilde{g}(m+t)=g(m)$ e $\widetilde{c}(m+t)=c(m)+\widetilde{c}(t)$. Tome $l \in \mathbb{N}$ tal que $m<k^{l}$. Então, para todo $x \in k^{l} \mathbb{N}, g(m+x)=g(m)$ e $c(m+x)=c(m)+c(x)$. Como $t \in \overline{k^{l} \mathbb{N}}$, então $\widetilde{g}(m+t)=g(m)$ e $\widetilde{c}(m+t)=c(m)+\widetilde{c}(t)$.

Agora, para cada $j \in \mathbb{N}$, considere a projeção nas $j$ primeiras coordenadas $\gamma_{j}$ definida para cada $\left(a_{n}\right)_{n \in \omega} \in\{0, \ldots, k-1\}^{\omega}$ por $\gamma_{j}\left(\left(a_{n}\right)_{n \in \omega}\right)=\left(a_{0}, \ldots, a_{j}\right)$. Note que, dado $s \in \beta \mathbb{N}, \gamma_{j}^{-1}\left[\left\{\gamma_{j}(\widetilde{f}(s))\right\}\right]$ é uma vizinhança básica de $\widetilde{f}(s)$ (pela topologia produto), logo por $1.26(\mathrm{~h}), \mathbb{N} \cap \widetilde{f}^{-1}\left[\gamma_{j}^{-1}\left[\left\{\gamma_{j}(\widetilde{f}(s))\right\}\right]\right]=$ $\left(\gamma_{j} \circ f\right)^{-1}\left[\left\{\gamma_{j}(\widetilde{f}(s))\right\}\right] \in s$. Para facilitar, denotaremos esse resultado por: para cada $s \in \beta \mathbb{N}$, $\left(\gamma_{j} f\right)^{-1} \gamma_{j}(\widetilde{f}(s)) \in s$.

Considere, a partir de agora, $\left(a_{n}\right)_{n \in \omega}=\widetilde{f}(q)$. Primeiramente, veremos que $\left(a_{n}\right)_{n \in \omega}$ não é eventualmente 0. Suponha, por absurdo, que seja, e tome $n_{0} \in \mathbb{N}$ tal que para todo $n \geq n_{0}$, $a_{n}=0$. Considere $m=\sum_{n=0}^{n_{0}-1} a_{n} k^{n}$. Seja $s \in \beta \mathbb{N}$ tal que $\{-m+A: A \in q\} \subset s$. Vale que $q=m+s$, pois $q \subset m+s$. Veremos que $s \in \bigcap_{n \in \mathbb{N}} \overline{k^{n} \mathbb{N}}$. Seja $l \geq n_{0}$. Tome $n \in\left\{0, \ldots, k^{l}-1\right\}$ tal que $n+k^{l} \mathbb{N} \in q$. Note que $\gamma_{l-1} f\left[n+k^{l} \mathbb{N}\right]=\left\{\gamma_{l-1}(n)\right\}$, logo $\gamma_{l-1} \tilde{f}(q)=\gamma_{l-1}(n)$. Porém, temos que $\gamma_{l-1} \tilde{f}(q)=\gamma_{l-1} f(m)$. Como $m, n<k^{l}$, tem-se que $m=n$. Dessa forma, $m+k^{l} \mathbb{N} \in q, \operatorname{logo}-m+\left(m+k^{l} \mathbb{N}\right)=k^{l} \mathbb{N} \in s$. Assim, $s \in \bigcap_{l \geq n_{0}} \overline{k^{l} \mathbb{N}} \subset \bigcap_{n \in \mathbb{N}} \overline{k^{n} \mathbb{N}}$. Dado $r \in \overline{k \mathbb{N}}, r+m+s+p=r+q+p=q+r+p=m+s+r+p . r+m+s+p=m+r+s+p$, $\operatorname{logo} m+r+s+p=m+s+r+p$, e, pelo lema 2.14, $r+s+p=s+r+p$. Note que pelo teorema 1.34, $\bigcap_{n \in \mathbb{N}} \overline{k^{n} \mathbb{N}}$ é um subsemigrupo. Logo $s+p \in \bigcap_{n \in \mathbb{N}} \overline{k^{n} \mathbb{N}}$. Agora seja $r \in\left(\left\{k^{n}: n \in \mathbb{N}\right\}\right)^{*}$ 
qualquer. Observe que $r \in \bigcap_{n \in \mathbb{N}} \overline{k^{n} \mathbb{N}}$, logo $r+p \in \bigcap_{n \in \mathbb{N}} \overline{k^{n} \mathbb{N}}$; e que, como $g$ é a identidade sobre $\left\{k^{n}: n \in \mathbb{N}\right\}$, então $\widetilde{g}(r)=r$. Deste modo, temos então (usando o que foi observado acima sobre $\widetilde{g}): r=\widetilde{g}(r)=\widetilde{g}(r+s+p)=\widetilde{g}(s+r+p)=\widetilde{g}(s)$. Uma contradição, pois temos $2^{\mathfrak{c}}$ possibilidades para $r \in\left(\left\{k^{n}: n \in \mathbb{N}\right\}\right)^{*}$.

Agora veremos que $\left(a_{n}\right)_{n \in \omega}$ não é eventualmente $k-1$. Suponha por absurdo que $n_{0} \in \mathbb{N}$ é tal que $a_{n}=k-1$ se $n \geq n_{0}$. Considere $m=-\sum_{n=0}^{n_{0}-1} a_{n} k^{n}+k^{n_{0}}$ e defina $t=m+q$. Seja $l>n_{0}$. Lembre que $\left(\gamma_{l} f\right)^{-1} \gamma_{l}(\widetilde{f}(q))=\left(\gamma_{l} f\right)^{-1} \gamma_{l}\left(\left(a_{n}\right)_{n \in \omega}\right) \in q$. Dado $x \in\left(\gamma_{l} f\right)^{-1} \gamma_{l}\left(\left(a_{n}\right)_{n \in \omega}\right)$, note que $x=\sum_{n=0}^{n_{0}-1} a_{n} k^{n}+\sum_{n=n_{0}}^{l}(k-1) k^{n}+\sum_{n>l} f(x)_{n} k^{n}$, de modo que $m+x=k^{n_{0}}+$ $k^{l+1}-k^{n_{0}}+\sum_{n>l} f(x)_{n} k^{n}=k^{l+1}+\sum_{n>l} f(x)_{n} k^{n} \in k^{l+1} \mathbb{N}$. Destarte, $m+q \in \overline{k^{l+1} \mathbb{N}}$; ou seja, $t \in \bigcap_{l>n_{0}} \overline{k^{l+1} \mathbb{N}} \subset \bigcap_{n \in \mathbb{N}} \overline{k^{n} \mathbb{N}}$. Temos então que dado $r \in \overline{k \mathbb{N}}, r+t+p=r+m+q+p=$ $m+r+q+p=m+q+r+p=t+r+p$, e, como no parágrafo acima, uma contradição.

Considere $L=\left\{n \in \omega: 0<a_{n}<k-1\right\}$. Mostraremos que $L$ é finito. Suponha que $L$ seja infinito, e tome $r \in\left(\left\{k^{n}: n \in L\right\}\right)^{*}$. Note que $c\left[\left\{k^{n}: n \in L\right\}\right]=\{1\}$, logo $\widetilde{c}(r)=1$. Além disso, dados $l \in L$ e $x \in\left(\gamma_{l} f\right)^{-1} \gamma_{l}\left(\left(a_{n}\right)_{n \in \omega}\right)$, note que $f(x)_{l}=a_{l}$, logo $0<f(x)_{l}<k-1$. Assim, vale que $c\left(k^{l}+x\right)=c(x)$, pois somar $k^{l}$ a $x$ não acrescenta nem retira coordenadas nulas da expansão em base $k$ de $x$. Portanto, temos que $\widetilde{c}(r+q)=\widetilde{c}(q)$. Usando as observações sobre $\widetilde{c}$, temos: $\widetilde{c}(q)+\widetilde{c}(p)=\widetilde{c}(r+q)+\widetilde{c}(p)=\widetilde{c}(r+q+p)=\widetilde{c}(q+r+p)=\widetilde{c}(q)+\widetilde{c}(r+p)=\widetilde{c}(q)+\widetilde{c}(r)+\widetilde{c}(p)=$ $\widetilde{c}(q)+1+\widetilde{c}(p)=\widetilde{c}(q)+\widetilde{c}(p)+1$. Contradição, pelo teorema 1.26(a), pois a função $h: \mathbb{N} \rightarrow \mathbb{N}$, $h(n)=n+1$ não possui pontos fixos.

Assim, temos que $L$ é finito, e $\left(a_{n}\right)_{n \in \omega}$ não é eventualmente $k-1$ ou eventualmente 0 . Portanto, vale que o conjunto $M=\left\{n \in \omega: a_{n}=k-1\right.$ e $\left.a_{n+1}=0\right\}$ é infinito. Tome então $r \in\left(\left\{k^{n}: n \in M\right\}\right)^{*}$. Note desta vez que, dados $m \in M$ e $x \in\left(\gamma_{m+1} f\right)^{-1} \gamma_{m+1}\left(\left(a_{n}\right)_{n \in \omega}\right)$, vale que $f(x)_{m}=a_{m}=k-1$ e $f(x)_{m+1}=0$. Logo $f\left(k^{m}+x\right)_{m}=0, f\left(k^{m}+x\right)_{m+1}=1$ e $f\left(k^{m}+x\right)_{i}=f(x)_{i}$ para $i \in \omega \backslash\{m, m+1\}$. Portanto, vale que $c\left(k^{m}+x\right)=c(x)$. Como antes, obtemos que $\widetilde{c}(q)+\widetilde{c}(p)=\widetilde{c}(q)+\widetilde{c}(p)+1$, uma contradição.

Antes do próximo lema, precisaremos de um resultado sobre semigrupos (um lema para o lema).

Lema 2.31. Seja $S$ um semigrupo topológico à direita compacto. Seja L ideal à esquerda minimal. Então cada $s \in L$ possui uma única identidade à esquerda em $L$.

Demonstração. Unicidade: Seja $s \in L$ e suponha que $a, b \in L$ são tais que $s=a s=b s$. Como, pelos teoremas 1.15(c) e 1.14, $K(S)=\bigcup\{R: R$ é ideal à direita minimal de $S\}$, união disjunta, tome $R_{a}$ e $R_{b}$ ideais à direita minimais tais que $a \in R_{a}$ e $b \in R_{b}$. Então $a s=b s \in R_{a} \cap R_{b}$, de forma que $R_{a}=R_{b}=: R$. Assim, $s=a s=b s \in R \cap L$ que é um grupo (teorema 1.17(b)), logo cancelando $s$ à direita, obtemos $a=b$.

Existência: note que $s \in R$ para algum ideal à direita minimal, $\operatorname{logo} s \in R \cap L$ que é grupo, e 
portanto $s$ possui uma identidade.

Lema 2.32. Seja $\phi: \beta \mathbb{N} \rightarrow \mathbb{N}^{*}$ um homomorfismo contínuo. Então $\phi(q)=\phi\left(q^{\prime}\right)$ para $q, q^{\prime}$ idempotentes quaisquer. Além disso, existe $m \in \mathbb{N}$ tal que para todo $x \geq m$ em $\mathbb{N}, \phi(x)=\phi(x)+\phi(q)$ (onde q é um idempotente).

Demonstração. Seja $p$ um idempotente. Temos que: $\phi(1)+\phi(p)=\phi(1+p)=\phi(p+1)=\phi(p)+\phi(1)$; $\phi(1)+\phi(p) \in \beta \mathbb{N}+\phi(p)=\overline{\mathbb{N}}+\phi(p)=\overline{\mathbb{N}+\phi(p)} ;$ e $\phi(p)+\phi(1) \in \phi[\beta \mathbb{N}]+\phi(1)=\phi[\overline{\mathbb{N}}]+\phi(1)=$ $\overline{\phi[\mathbb{N}]+\phi(1)}$. Logo, pelo 1.26(c), $(\mathbb{N}+\phi(p)) \cap \overline{\phi[\mathbb{N}]+\phi(1)} \neq \varnothing$ ou $\overline{\mathbb{N}+\phi(p)} \cap(\phi[\mathbb{N}]+\phi(1)) \neq \varnothing$. Na primeira possibilidade, existe $k \in \mathbb{N}$ tal que $k+\phi(p) \in \overline{\phi[\mathbb{N}]+\phi(1)} \subset \overline{\phi[\beta \mathbb{N}]}=\phi[\beta \mathbb{N}]$ (pois $\phi$ é contínua). Logo, para cada $m \in \mathbb{N}, k m+\phi(p) \in \beta \mathbb{N}$, pois: $k m+\phi(p)=k(m-1)+k+\phi(p)+\phi(p)=$ $k(m-1)+\phi(p)+k+\phi(p) \in \phi[\beta \mathbb{N}]+\phi[\beta \mathbb{N}] \subset \phi[\beta \mathbb{N}]$. Dessa forma, $k \mathbb{N}+\phi(p) \subset \phi[\beta \mathbb{N}], \operatorname{logo}$ $\overline{k \mathbb{N}}+\phi(p) \subset \overline{k \mathbb{N}+\phi(p)} \subset \phi[\beta \mathbb{N}]$. Ou seja, dado $r \in \overline{k \mathbb{N}}, r+\phi(p) \in \phi[\beta \mathbb{N}]$ e portanto comuta com $\phi(1), \log o r+\phi(1)+\phi(p)=r+\phi(p)+\phi(1)=\phi(1)+r+\phi(p)$, contradizendo o lema 2.30 .

Portanto somos forçados a ter $\overline{\mathbb{N}+\phi(p)} \cap(\phi[\mathbb{N}]+\phi(1)) \neq \varnothing$. Ou seja, temos $k \in \mathbb{N}$ tal que $\phi(k)+\phi(1)=\phi(k+1) \in \overline{\mathbb{N}+\phi(p)}=\beta \mathbb{N}+\phi(p)$. Defina $m=k+1$. Note que $\phi(m)=\phi(m)+$ $\phi(p)=\phi(m+p) \in \mathbb{N}^{*}$. Além disso, dado $x>m, \phi(x)=\phi(x-m)+\phi(m) \in \beta \mathbb{N}+\phi(p)$ e $\operatorname{logo}$ $\phi(x)=\phi(x)+\phi(p)$. Como $\phi[\{x \in \mathbb{N}: x \geq m\}] \subset \beta \mathbb{N}+\phi(p)$, segue que $\phi\left[\mathbb{N}^{*}\right] \subset \beta \mathbb{N}+\phi(p)$, e assim $\phi(t)=\phi(t)+\phi(p)$ para todo $t \in \mathbb{N}^{*}$.

Dessa forma, demonstramos que $\phi\left[\mathbb{N}^{*}\right]=\phi\left[\mathbb{N}^{*}\right]+\phi(p)$, para um idempotente $p$ qualquer. Suponha que $u$ seja algum idempotente de $\phi\left[\mathbb{N}^{*}\right]$. Vale que $\phi^{-1}[\{u\}]$ é um subsemigrupo compacto de $\beta \mathbb{N}$, logo possui um idempotente $p$. Temos então que $u=\phi(p)$. Ou seja, todo idempotente de $\phi\left[\mathbb{N}^{*}\right]$ é da forma $\phi(p)$ para $p$ idempotente. Assim, como qualquer ideal à esquerda minimal de $\phi\left[\mathbb{N}^{*}\right]$ é da forma $\phi\left[\mathbb{N}^{*}\right]+u$ com $u$ idempotente, segue que $\phi\left[\mathbb{N}^{*}\right]$ é seu próprio ideal à esquerda minimal. Usaremos agora o lema 2.31: sejam $q, q^{\prime}$ idempotentes. Observe que $\phi(q)+\phi(m)=\phi(m)+\phi(q)$ e, pelo que provamos acima, $\phi(m)+\phi(q)=\phi(m)$; analogamente, $\phi\left(q^{\prime}\right)+\phi(m)=\phi(m)$. Assim, $\phi(q)=\phi\left(q^{\prime}\right)$.

Nesse ponto, já podemos afirmar que $\phi\left[\mathbb{N}^{*}\right]$ é um grupo, pois é topológico à direita compacto, simples à esquerda, e possui um único idempotente.

Para o próximo lema, note que, dado $p \in \mathbb{N}^{*}, p$ é o limite de uma rede em $\mathbb{N}$, e vale que tal rede está eventualmente na vizinhança $\beta \mathbb{N} \backslash\{1, \ldots, l\}$, para todo $l \in \mathbb{N}$.

Lema 2.33. Seja $\phi: \beta \mathbb{N} \rightarrow \beta \mathbb{N}$ contínua. Suponha que existem $m \in \mathbb{N}$ e $p \in \mathbb{N}^{*}$ tais que $\phi(p) \notin \phi[\mathbb{N}]$ e $\phi(x)=\phi(x+p)$ para todos $x \geq m$ em $\mathbb{N}$. Então existe uma sequência crescente $\left(n_{k}\right)_{k \geq 0}$ em $\mathbb{N}$ e uma sequência decrescente $\left(W_{k}\right)_{k \geq 0}$ de vizinhanças clopens de $\phi(p)$ tais que $\phi\left(n_{k_{1}}+\cdots+n_{k_{r}}\right) \in$ $W_{k_{1}} \backslash W_{k_{1}+1}$ sempre que $k_{1}<\ldots<k_{r}$. 
Demonstração. Construiremos por recursão. No primeiro passo, defina $n_{0}=m, W_{0}=\beta \mathbb{N}$ e tome $W_{1}$ qualquer vizinhança clopen de $\phi(p)$ tal que $\phi\left(n_{0}\right) \notin W_{1}$.

Suponha então construídos $n_{0}, \ldots, n_{k}$ e $W_{0}, \ldots, W_{k+1}$ com as propriedades desejadas. Note que, dados $k_{1}<\ldots<k_{r} \leq k, \phi\left(n_{k_{1}}+\cdots+n_{k_{r}}+p\right)=\phi\left(n_{k_{1}}+\cdots+n_{k_{r}}\right) \in W_{k_{1}} \backslash W_{k_{1}+1}$, sendo este um aberto. Como $p$ é o limite de uma rede em $\mathbb{N}, p \in \mathbb{N}^{*}, \phi$ é contínua, $\phi\left(n_{k_{1}}+\cdots+n_{k_{r}}+p\right) \in W_{k_{1}} \backslash W_{k_{1}+1}$ e $\phi(p) \in W_{k+1}$, então existe $n_{k+1}>n_{k}$ tal que $\phi\left(n_{k+1}\right) \in W_{k+1}$ e $\phi\left(n_{k_{1}}+\cdots+n_{k_{r}}+n_{k+1}\right) \in$ $W_{k_{1}} \backslash W_{k_{1}+1}$ sempre que $k_{1}<\ldots<k_{r} \leq k$ (pois há finitas possibilidades para $k_{1}, \ldots, k_{r} \leq k$ ). Tome então $W_{k+2} \subset W_{k+1}$ vizinhança clopen de $\phi(p)$ tal que $\phi\left(n_{k+1}\right) \notin W_{k+2}$.

Assim, temos: se $k_{1}<\ldots<k_{r} \leq k, \phi\left(n_{k_{1}}+\cdots+n_{k_{r}}\right) \in W_{k_{1}} \backslash W_{k_{1}+1}$ e $\phi\left(n_{k_{1}}+\cdots+n_{k_{r}}+n_{k+1}\right) \in$ $W_{k_{1}} \backslash W_{k_{1}+1}$; e $\phi\left(n_{k+1}\right) \in W_{k+1} \backslash W_{k+2}$. Ou seja, $\phi\left(n_{k_{1}}+\cdots+n_{k_{r}}\right) \in W_{k_{1}} \backslash W_{k_{1}+1}$ sempre que $k_{1}<\ldots<k_{r} \leq k+1$. Está feito o passo da recursão.

Para os próximos lema e teorema, usaremos a seguinte notação: dado $X \subset \mathbb{N}$, $F S(X)=\left\{\sum_{a \in F} a: F \in \mathcal{P}_{f}(X)\right\}$ e $F S_{n}(X)=\left\{\sum_{a \in F} a: F \in \mathcal{P}_{f}(X)\right.$ e min $\left.F \geq n\right\}$. Além disso, para tornar a notação mais limpa, escreveremos $\Sigma F$ no lugar de $\sum_{a \in F} a$.

Lema 2.34. Assuma as hipóteses do lema anterior. Então existem $B, C \subset \mathbb{N}$ infinitos e disjuntos tais que $\phi[\overline{F S(B)}] \cap \phi[\overline{F S(C)}]=\varnothing$.

Demonstração. Tome pelo lema anterior as sequências $\left(n_{k}\right)_{k \geq 0}$ e $\left(W_{k}\right)_{k \geq 0}$. Tome $B$ e $C$ subconjuntos infinitos disjuntos de $\left\{n_{k}: k \geq 0\right\}$. Veremos que se $x \in F S(B)$, então $\phi(x) \notin \overline{\phi[F S(C)]}$. Escreva $x=\Sigma F$, com $F \in \mathcal{P}_{f}(B)$ e $\min F=n_{k}$. Temos então que $\phi(x) \in W_{k} \backslash W_{k+1}$. Por outro lado, dado $y \in F S(C)$ com $y=\Sigma G, G \in \mathcal{P}_{f}(C)$, se $\min G<n_{k}$, então $\phi(y) \notin W_{k}$ (lembre que a sequência $\left(W_{k}\right)_{k \geq 0}$ é decrescente); e se $\min G>n_{k}$, então $\phi(y) \in W_{k+1}$. Como $W_{k}$ e $W_{k+1}$ são clopens, segue que $\overline{\phi[F S(C)]} \subset \beta \mathbb{N} \backslash W_{k} \cup W_{k+1}$, e portanto $\phi(x) \notin \overline{\phi[F S(C)]}$.

Analogamente, dado $y \in F S(C), \phi(y) \notin \overline{\phi[F S(B)]}$. Desse modo, temos que $\phi[F S(B)] \cap \overline{\phi[F S(C)]}=$ $\phi[F S(C)] \cap \overline{\phi[F S(B)]}=\varnothing$, logo, pelo teorema 1.26(c), como $F S(B)$ e $F S(C)$ são enumeráveis, $\overline{\phi[F S(B)]} \cap \overline{\phi[F S(C)]}=\varnothing$. Ou seja, $\phi[\overline{F S(B)}] \cap \phi[\overline{F S(C)}]=\varnothing$, como queríamos.

Assim, podemos provar o teorema principal desta sub-seção, provando a seguinte (surpreendente) afirmação:

Teorema 2.35. Seja $\phi: \beta \mathbb{N} \rightarrow \mathbb{N}^{*}$ um homomorfismo contínuo. Então $\phi[\beta \mathbb{N}]$ é finito.

Demonstração. Suponha que $\phi[\beta \mathbb{N}]$ é infinito. Vale que $\phi[\mathbb{N}]$ não possui idempotentes: se $\phi(k)$ fosse um idempotente para algum $k \in \mathbb{N}$, por indução teríamos que $\phi(a \cdot k)=\phi(k)$ para todo $a \in \mathbb{N}$. Assim, dado $x \in \mathbb{N}$, se $x \geq k$, então $x=a \cdot k+b$, com $a \in \mathbb{N}$ e $b<k$. Logo $\phi(x)=$ $\phi(a \cdot k)+\phi(b)=\phi(k)+\phi(b)$. Portanto $\phi[\mathbb{N}]=\{\phi(x): x<k\} \cup\{\phi(k)+\phi(b): b<k\}$, que é finito, $\operatorname{logo} \overline{\phi[\mathbb{N}]}=\phi[\beta \mathbb{N}]=\{\phi(x): x<k\} \cup\{\phi(k)+\phi(b): b<k\}$. 
Dessa forma, dado $p$ idempotente, as hipóteses do lema 2.34 estão satisfeitas, de acordo com o lema 2.32, então temos $B, C \subset \mathbb{N}$ infinitos e disjuntos tais que $\phi[\overline{F S(B)}] \cap \phi[\overline{F S(C)}]=\varnothing$. Como, pelo teorema 1.34, $\bigcap_{n \geq 1} \overline{F S_{n}(B)}$ e $\bigcap_{n \geq 1} \overline{F S_{n}(C)}$ são subsemigrupos, cada um possui um idempotente; sejam $s \in \bigcap_{n \geq 1} \overline{F S_{n}(B)}$ e $t \in \bigcap_{n \geq 1} \overline{F S_{n}(C)}$ tais idempotentes. Temos então que $\phi(s) \neq \phi(t)$, contradizendo o lema 2.32 .

Corolário 2.36. Seja $\phi: \beta \mathbb{N} \rightarrow \mathbb{N}^{*}$ um homomorfismo contínuo. Então $\phi\left[\mathbb{N}^{*}\right] \cong \mathbb{Z}_{m}$ para algum $m \in \mathbb{N}$.

Demonstração. Afirmamos que $\phi\left[\mathbb{N}^{*}\right]$ é um grupo cíclico de gerador $\phi(1)+\phi(p)$, para $p$ um idempotente qualquer.

Note que dados $k, l \in \mathbb{N},(\phi(k)+\phi(p))+(\phi(l)+\phi(p))=\phi(k+l)+\phi(p)$. Já sabemos que $\phi\left[\mathbb{N}^{*}\right]$ é um grupo, e pelo teorema anterior ele é finito. Seja $h$ a identidade desse grupo. Note que $\{\phi(n)+\phi(p): n \in \mathbb{N}\} \subset \phi\left[\mathbb{N}^{*}\right]$, e portanto existe o menor $n>1$ tal que $\phi(n)+\phi(p)=\phi(k)+\phi(p)$, com $k<n$. Vale então que $\phi(n)+\phi(p)=\phi(n-k)+\phi(p)+\phi(k)+\phi(p)=\phi(k)+\phi(p)$. Aplicando a inversa de $\phi(k)+\phi(p)$, temos que $\phi(n-k)+\phi(p)=h$. Defina $m=n-k$. Temos então, por indução, que $\phi(k \cdot m)+\phi(p)=\phi(m)+\phi(p)=h$ para todo $k \in \mathbb{N}$. Segue disso que se $x \geq m$ e escrevemos $x=a \cdot m+b \operatorname{com} a \geq 1$ e $0 \leq b<m$, então $\phi(x)+\phi(p)=\phi(a \cdot m)+\phi(p)+\phi(b)+\phi(p)=\phi(b)+\phi(p)$. Ou seja, dado $i \in\{0, \ldots, m-1\}$, e $x \in i+m \mathbb{N}, \phi(x)+\phi(p)=\phi(i)+\phi(p)$. Agora seja $\phi(q) \in \phi\left[\mathbb{N}^{*}\right]$. Vimos no lema 2.32 que $\phi\left[\mathbb{N}^{*}\right]=\phi\left[\mathbb{N}^{*}\right]+\phi(p)$, logo $\phi(q)=\phi(q)+\phi(p)$. Tome $i \in\{0, \ldots, m-1\}$ tal que $i+m \mathbb{N} \in \phi(q)$. Pelo que foi observado acima, e como $\phi$ e a soma à direita são contínuas, $\phi(q)=\phi(q)+\phi(p)=\phi(i)+\phi(p)$. Portanto, temos que $\phi\left[\mathbb{N}^{*}\right]=\{\phi(i)+\phi(p): 1 \leq i \leq m\}$, com $\phi(m)+\phi(p)$ a identidade do grupo. Portanto, $\phi\left[\mathbb{N}^{*}\right]$ é isomorfo ao grupo cíclico de ordem $m, \mathbb{Z}_{m}$. (Para ver que de fato $|\{\phi(i)+\phi(p): 1 \leq i \leq m\}|=m$, suponha $1 \leq i<j \leq m$ com $\phi(i)+\phi(p)=\phi(j)+\phi(p)$; observe que $j \geq n>n-k \geq m$, contradição.)

Assim, a imagem de qualquer homomorfismo contínuo entre $\beta \mathbb{N}$ e $\mathbb{N}^{*}$ é finita. Tem-se a questão: é possível que seja sempre trivial (unitária)? Unindo os resultados do teorema 2.35 e do lema 2.32, temos que a existência de um homomorfismo contínuo não trivial é equivalente à existência de um elemento de ordem finita em $\beta \mathbb{N}$ que não seja um idempotente (ou seja, ordem maior ou igual a 2).

De fato, o Teorema 3.1 do artigo de Neil Hindman [3] dá a seguinte forma mais precisa a essa equivalência:

Teorema 2.37. São equivalentes:

(a) Existe um homomorfismo contínuo não-trivial de $\beta \mathbb{N}$ em $\mathbb{N}^{*}$; 
(b) Existe um grupo finito não-trivial em $\mathbb{N}^{*}$, ou existem $p, q \in \mathbb{N}^{*}$ distintos tais que $q+q=q=$ $p+p=q+p=p+q$.

Demonstração. (a) $\Rightarrow(\mathrm{b})$ :

Seja $\phi: \beta \mathbb{N} \rightarrow \mathbb{N}^{*}$ um homomorfismo contínuo não-trivial. Pelo teorema $2.35, \phi\left[\mathbb{N}^{*}\right]$ é um grupo finito, logo se $\left|\phi\left[\mathbb{N}^{*}\right]\right|>1$ ele é não-trivial. Suponha então $\left|\phi\left[\mathbb{N}^{*}\right]\right|=\{q\}$ ( $q$ idempotente). Pelo lema 2.32 , tome $m \in \mathbb{N}$ o menor tal que se $x \geq m, \phi(x)=\phi(x)+\phi(p) \in \phi\left[\mathbb{N}^{*}\right]$. Como $\phi$ não é trivial, temos que $m \geq 2$, $\operatorname{logo}$ defina $p=\phi(m-1)$. Temos então que $p+p=\phi(m-1+m-1)=q$, $q+p=\phi(m+m-1)=q$ e $p+q=\phi(m-1+m)=q$.

(b) $\Rightarrow(\mathrm{a})$ :

Suponha que existe um grupo finito não trivial em $\mathbb{N}^{*}$; então algum subgrupo dele é homeomorfo a um grupo cíclico $\mathbb{Z}_{n}, n>1$. Ou seja, existe um homomorfismo injetor $\psi: \mathbb{Z}_{n} \rightarrow \mathbb{N}^{*}$. Defina $\phi: \mathbb{N} \rightarrow \mathbb{N}^{*}$ por $\phi(k)=\psi(\bar{k})$. Temos então que $\widetilde{\phi}: \beta \mathbb{N} \rightarrow \mathbb{N}^{*}$ é um homomorfismo contínuo não-trivial.

Suponha agora que existem $p, q \in \mathbb{N}^{*}$ distintos tais que $q+q=q=p+p=q+p=p+q$. Então $\phi: \beta \mathbb{N} \rightarrow \mathbb{N}^{*}$ definida por $\phi(1)=p$ e $\phi(r)=q$ para todo $r \neq 1$, é um homomorfismo, pois, dados $r, s \neq 1: \phi(1+1)=q$ e $\phi(1)+\phi(1)=p+p=q ; r+1 \neq 1 \neq 1+r, \log 0 \phi(r+1)=q=\phi(1+r)$ e $\phi(r)+\phi(1)=q+p=q=p+q=\phi(1)+\phi(r) ; r+s \neq 1, \log 0 \phi(r+s)=q$ e $\phi(r)+\phi(s)=q+q=q$. Além disso, $\phi$ é contínua pois é constante sobre dois clopens, $\{1\}$ e $\beta \mathbb{N} \backslash\{1\}$.

Para os próximos corolários, definimos, para cada $q \in \beta \mathbb{N}, q_{1}=q$ e $q_{n+1}=q_{n}+q$.

Corolário 2.38. Se $\phi: \beta \mathbb{N} \rightarrow \mathbb{N}^{*}$ é um homomorfismo contínuo, então $\left|\phi^{2}[\beta \mathbb{N}]\right|=1$.

Demonstração. Primeiramente defina $q=\phi(1)$ e vejamos que para algum $n \in \mathbb{N}, q_{n}$ é idempotente. Lembre que pelo lema 2.32 , existe $m^{\prime} \in \mathbb{N}$ tal que se $x \geq m^{\prime}$ então $\phi(x)=\phi(x)+\phi(p) \in \phi\left[\mathbb{N}^{*}\right]$, em que $p$ é idempotente. Logo, como $\phi\left[\mathbb{N}^{*}\right] \cong \mathbb{Z}_{m}$ e $\phi\left(m^{\prime}\right) \in \phi\left[\mathbb{N}^{*}\right]$, tome $n \geq m^{\prime}$ tal que $\phi(n)+\phi(p)$ é a identidade de $\phi\left[\mathbb{N}^{*}\right]$. Temos, pois, que $\phi(n)=\phi(n)+\phi(p)$ é um idempotente. Usando que $\phi$ é homomorfismo, $\phi(n)=q_{n}$. Logo $q_{n}$ é idempotente.

Afirmação: $n \mathbb{N} \in q$. Para comprovar isso, seja $\gamma$ o homomorfismo canônico de $\mathbb{N}$ em $\mathbb{Z}_{n^{2}}$. Vale que $\widetilde{\gamma}$ é um homomorfismo também, e portanto $\widetilde{\gamma}\left(q_{n}\right)=0$. Por outro lado, note que $\widetilde{\gamma}\left(q_{n}\right)=n \cdot \widetilde{\gamma}(q)$, $\operatorname{logo} n \cdot \widetilde{\gamma}(q)=0$. Além disso, dado $x \in \mathbb{N}, n \cdot \gamma(x)=0$ se e somente se $x \in n \mathbb{N}$. Portanto $n \mathbb{N} \in q$. Temos então: $\phi(n)=q_{n}$. Como $q_{n}$ é idempotente, por indução vale que $\phi(k \cdot n)=q_{n}$ para todo $k \in \mathbb{N}$. Ou seja, $\phi[n \mathbb{N}]=\left\{q_{n}\right\}$, e portanto $\phi(q)=q_{n}$. Ou seja $\phi(\phi(1))=q_{n}$. Agora, aplicando indução, dado $l>1$, temos que $\phi(\phi(l))=\phi(\phi(l-1)+\phi(1))=\phi(\phi(l-1))+\phi(\phi(1))=q_{n}+q_{n}=q_{n}$. Ou seja, $\phi^{2}[\mathbb{N}]=\left\{q_{n}\right\}$, do que segue que $\phi^{2}[\beta \mathbb{N}]=\left\{q_{n}\right\}$. 
Corolário 2.39. Se $q \in \mathbb{N}^{*}$ possui ordem infinita, então $q$ não comuta com todo elemento de $\operatorname{cl}\left\{q_{n}: n \in \mathbb{N}\right\}$. Disso segue que qualquer elemento de $\mathbb{N}^{*}$ de ordem infinita não está no centro de qualquer subsemigrupo compacto de $\beta \mathbb{N}$.

Demonstração. Considere a função $\phi: \mathbb{N} \rightarrow\left\{q_{n}: n \in \mathbb{N}\right\}$ dada por $\phi(n)=q_{n}$. Vale que sua extensão $\widetilde{\phi}: \beta \mathbb{N} \rightarrow \operatorname{cl}\left\{q_{n}: n \in \mathbb{N}\right\}$ é sobrejetora (pois $\widetilde{\phi}[\beta \mathbb{N}]=\widetilde{\phi}[\operatorname{cl} \mathbb{N}]=\operatorname{cl} \phi[\mathbb{N}]$ ). Agora, se $q$ comutar com todo elemento de $\operatorname{cl}\left\{q_{n}: n \in \mathbb{N}\right\}, \widetilde{\phi}$ será um homomorfismo, pois: sejam $r, s \in \beta \mathbb{N}$, e tome $\left(m_{\alpha}\right)_{\alpha \in A}$ e $\left(n_{\beta}\right)_{\beta \in B}$ redes em $\mathbb{N}$ convergindo para $r$ e $s$, respectivamente. Então temos:

$$
\begin{aligned}
\widetilde{\phi}(r+s) & =\lim _{\alpha} \lim _{\beta} \phi\left(m_{\alpha}+n_{\beta}\right) \\
& =\lim _{\alpha} \lim _{\beta}\left(\phi\left(n_{\beta}\right)+\phi\left(m_{\alpha}\right)\right) \\
& =\lim _{\alpha}\left(\widetilde{\phi}(s)+\phi\left(m_{\alpha}\right)\right) \\
& =\lim _{\alpha}\left(\phi\left(m_{\alpha}\right)+\widetilde{\phi}(s)\right) \\
& =\widetilde{\phi}(r)+\widetilde{\phi}(s) .
\end{aligned}
$$

Ou seja, $\phi$ seria um homomorfismo contínuo de $\beta \mathbb{N}$ em $\mathbb{N}^{*}$ de imagem infinita, contradizendo o teorema 2.35 .

Notamos rapidamente que o lema 2.30 nos fornece uma prova de que o centro topológico de $\mathbb{N}^{*}$ é vazio.

Corolário 2.40. O centro topológico de $\mathbb{N}^{*}$ é vazio.

Demonstração. Suponha $q \in \Lambda\left(\mathbb{N}^{*}\right)$. Seja $p$ idempotente qualquer. Vale que $n+q+p=q+n+p$ para todo $n \in \mathbb{N}$. Logo, passando ao limite e usando que $q \in \Lambda\left(\mathbb{N}^{*}\right)$, temos que $r+q+p=q+r+p$ para todo $r \in \mathbb{N}^{*}$, contradizendo o lema 2.30 .

O Teorema 2 do artigo [7] dá uma caracterização dos elementos canceláveis à direita de $\beta \mathbb{N}$. Porém antes precisaremos de um lema, que é o Teorema 1 do artigo [8].

Lema 2.41. Suponha que $q \in \mathbb{N}^{*}$ não é cancelável à direita. Então existe $s \in \mathbb{N}^{*}$ tal que $q=s+q$.

Demonstração. Sejam $p_{1}, p_{2}$ distintos e tais que $p_{1}+q=p_{2}+q$. Tome $A_{1} \in p_{1}$ e $A_{2} \in p_{2}$ tais que $A_{1} \cap A_{2}=\varnothing$. Temos que $p_{1}+q \in \overline{A_{1}}+q=\overline{A_{1}+q}$, e $p_{2}+q \in \overline{A_{2}+q}$. Ou seja, $\overline{A_{1}+q} \cap \overline{A_{2}+q} \neq \varnothing$; como $A_{1}, A_{2} \subset \mathbb{N}$, temos que $\left(A_{1}+q\right) \cap \overline{A_{2}+q} \neq \varnothing$ ou $\overline{A_{1}+q} \cap\left(A_{2}+q\right) \neq \varnothing$. Sem perda de generalidade, tome $a \in A_{1}$ e $r \in \overline{A_{2}}$ tais que $a+q=r+q$. Tome $s \in \mathbb{N}^{*}$ tal que $s \supset\{-a+R: R \in r\}$. Vale então que $q=s+q$, pois: seja $Q \in q . a+Q \in a+q=r+q$, $\operatorname{logo}\{x \in \mathbb{N}:-x+a+Q \in q\} \in r$, $\log \mathrm{o}-a+\{x \in \mathbb{N}:-x+a+Q \in q\} \in s$, ou seja, $\{y \in \mathbb{N}:-(a+y)+a+Q \in q\} \in s$, portanto $\{y \in \mathbb{N}:-y+Q \in q\} \in s$, e $\operatorname{assim} Q \in s+q$. 
(Note que o lema acima vale ipsis litteris se considerarmos $G$ grupo enumerável no lugar de $\mathbb{N}$.)

Teorema 2.42. Seja $q \in \mathbb{N}^{*}$. $q$ é cancelável à direita se e somente se existe $X \in q$ com a seguinte propriedade: enumerando $X=\left\{x_{n}: n \in \mathbb{N}\right\}$ de forma crescente, para cada $k \in \mathbb{N}$ vale que $\left\{x_{n}: x_{n+1}-x_{n}>k\right\} \in q$.

Demonstração. $(\Rightarrow)$ : Por contraposição. Ou seja, suponha que para todo $X \in q$ existe um $k_{X} \in \mathbb{N}$ tal que $\left\{x_{n}: x_{n+1}-x_{n} \leq k_{X}\right\} \in q$ (sendo $X=\left\{x_{n}: n \in \mathbb{N}\right\}$ uma enumeração crescente). Como $\left\{x_{n}: x_{n+1}-x_{n} \leq k_{X}\right\}=\bigcup_{m=1}^{k_{X}}\left\{x_{n}: x_{n+1}-x_{n}=m\right\}$, então existe $m_{X}$ tal que $\left\{x_{n}: x_{n+1}-x_{n}=\right.$ $\left.m_{X}\right\} \in q$.

Assim, para cada $X=\left\{x_{n}: n \in \mathbb{N}\right\} \in q$, temos que $\left\{x_{n}: x_{n+1}-x_{n}=m_{X}\right\} \in q$. Assim, para cada $X \in q$, tome $\left(x_{\lambda}\right)_{\lambda \in \Lambda}$ rede em $\left\{x_{n}: x_{n+1}-x_{n}=m_{X}\right\} \subset X$ que converge pra $q$. Defina, para cada $x_{\lambda}, y_{\lambda}=m_{X}+x_{\lambda}$ (note que $y_{\lambda} \in\left\{x_{n}: x_{n+1}-x_{n}=m_{X}\right\} \subset X$ ). Temos então que $\lim _{\lambda \in \Lambda}\left(m_{X}+x_{\lambda}\right)=m_{X}+q$, ou seja, $\lim _{\lambda \in \Lambda} y_{\lambda}=m_{X}+q$, e logo $m_{X}+q \in \bar{X}$ para cada $X \in q$. Assim, temos que a rede $\left(m_{X}+q\right)_{X \in q}$ converge para $q$; tome uma sub-rede $\left(m_{X_{\mu}}\right)_{\mu \in M}$ de $\left(m_{X}\right)_{X \in q}$ que converge, digamos, para $s$. Então temos que $q=\lim _{\mu \in M}\left(m_{X_{\mu}}+q\right)=\lim _{\mu \in M}\left(m_{X_{\mu}}\right)+q=s+q$. Assim, $q$ não é cancelável à direita (pois se fosse, $q=s+q \Rightarrow 1+q=1+s+q \Rightarrow 1=1+s$, absurdo).

$(\Leftarrow)$ : Por contraposição, isto é, suponha que $q$ não é cancelável à direita. Então pelo lema acima, $q=s+q$ para algum $s \in \mathbb{N}^{*}$. Dado $X \in q, X \in s+q$, logo existem $Y \in s$ e $\left\{X_{y}: y \in Y\right\} \subset q$ tais que $\bigcup_{y \in Y} y+X_{y} \subset X$. Enumere $X=\left\{x_{n}: n \in \mathbb{N}\right\}$ de forma crescente. Dado $x_{n} \in X_{y}$, $y+x_{n} \in X$; como a enumeração é crescente, temos que $y+x_{n} \geq x_{n+1}$, $\operatorname{logo} x_{n+1}-x_{n} \leq y$. Ou seja, $\left\{x_{n}: x_{n+1}-x_{n} \leq y\right\} \supset X \cap X_{y} \in q$.

Lembre que definimos $q_{1}=q$ e $q_{n+1}=q_{n}+q$. Com a caracterização dada acima, podemos provar o seguinte:

Teorema 2.43. Se $q \in \mathbb{N}^{*}$ é cancelável à direita, então $\overline{\left\{q_{n}: n \in \mathbb{N}\right\}}$ não é um subsemigrupo.

Demonstração. Por absurdo. Suponha que $\overline{\left\{q_{n}: n \in \mathbb{N}\right\}}$ seja um subsemigrupo. Como $q$ é cancelável à direita, pelo teorema acima tome $X \in q$ tal que, enumerando $X=\left\{x_{n}: n \in \mathbb{N}\right\}$ de forma crescente, $\left\{x_{n}: x_{n+1}-x_{n}>m\right\} \in q$ para todo $m \in \mathbb{N}$.

Considere somas da forma $x_{n_{1}}+\cdots+x_{n_{k}}$, tais que $n_{1}<\ldots<n_{k}$ e $x_{n_{r}+1}-x_{n_{r}}>\sum_{i=1}^{r-1} x_{n_{i}}$ para todo $r \in\{1, \ldots, k\}$. Vejamos que somas desse tipo são únicas. Suponha $x_{n_{1}}+\cdots+x_{n_{k}}=$ $x_{m_{1}}+\cdots+x_{m_{s}}$ duas somas desse tipo. Vale que $n_{k}=m_{s}$, pois se s.p.g. $n_{k}>m_{s}$, então $x_{n_{k}}-x_{m_{s}} \geq x_{m_{s}+1}-x_{m_{s}}>\sum_{i=1}^{s-1} x_{m_{i}}$ e $\operatorname{logo} x_{n_{k}}>x_{m_{1}}+\cdots+x_{m_{s}}$, absurdo. Ou seja, $x_{n_{k}}=x_{m_{s}}$, logo ambos podem ser cancelados na igualdade; assim podemos aplicar uma indução em $k$, e ter que $k=s$ e $n_{i}=m_{i}$ para cada $i \in\{1, \ldots, k\}$.

Desse modo, seja $S$ o conjunto de todas as somas desse tipo, e defina, para cada $m \in \mathbb{N}, S_{m}$ o 
subconjunto de $S$ das somas $x_{n_{1}}+\cdots+x_{n_{k}}$ que também verificam $x_{n_{i}+1}-x_{n_{i}}>m$ para cada $i \in\{1, \ldots, k\}$. Definimos também $c: S \rightarrow \mathbb{N}$ por $c\left(x_{n_{1}}+\cdots+x_{n_{k}}\right)=k$.

Mostraremos por indução em $r$ que, para cada $m \in \mathbb{N},\left\{s \in S_{m}: c(s)=r\right\} \in q_{r}$. No caso $r=1$, temos que $\left\{s \in S_{m}: c(s)=1\right\}=\left\{x_{n_{1}}: x_{n_{1}+1}-x_{n_{1}}>m\right\} \in q$. Suponha que valha para $r-1$. Mostraremos que para cada $U \in q_{r}, U \cap\left\{s \in S_{m}: c(s)=r\right\} \neq \varnothing$. Como $U \in q_{r}=q_{r-1}+q$, então existem $V \in q_{r-1}$ e $\left\{W_{v}: v \in V\right\} \subset q$ tais que $\bigcup_{v \in V} v+W_{v} \subset U$. Usando a hipótese indutiva, tome $v \in V \cap S_{m}$ tal que $c(v)=r-1$. Como $W_{v} \cap X \in q \in \mathbb{N}^{*}$ e usando a propriedade de $X$, tome $x_{n} \in W_{v} \cap X$ tal que $x_{n+1}-x_{n}>v, x_{n+1}-x_{n}>m$ e $x_{n}>v$. Vale então que $v+x_{n} \in S_{m} \cap U$ e $c\left(v+x_{n}\right)=r$, como queríamos.

Portanto, considerando $\widetilde{c}: \bar{S} \rightarrow \beta \mathbb{N}$, temos que $\widetilde{c}\left(q_{r}\right)=r$.

Agora, para cada $m \in \mathbb{N}$, considere $T_{m}=\left\{x_{n_{1}}+\cdots+x_{n_{k}} \in S_{m}: x_{n_{1}}>m\right.$ e $x_{n_{r}+1}-x_{n_{r}}>$ $\left.m+\sum_{i=1}^{r-1} x_{n_{i}}\right\}$. Vejamos primeiro que $T_{m} \in q$. Vimos acima que $\left\{s \in S_{m}: c(s)=1\right\} \in q$, e $\left\{s \in S_{m}: c(s)=1\right\}=\left\{x_{n_{1}}: x_{n_{1}+1}-x_{n_{1}}>m\right\}$. Como $q \in \mathbb{N}^{*}$ segue que $\left\{x_{n_{1}}: x_{n_{1}+1}-x_{n_{1}}>m\right.$ e $\left.x_{n_{1}}>m\right\} \in q$. Note que $\left\{x_{n_{1}}: x_{n_{1}+1}-x_{n_{1}}>m\right.$ e $\left.x_{n_{1}}>m\right\} \subset T_{m}$ (são exatamente os elementos de $T_{m}$ de comprimento 1$)$.

Portanto, $q \in \bigcap_{m \in \mathbb{N}} \overline{T_{m}}$, que é compacto. Queremos ver que $\overline{\left\{q_{n}: n \in \mathbb{N}\right\}} \subset \bigcap_{m \in \mathbb{N}} \overline{T_{m}}$; basta ver que $\bigcap_{m \in \mathbb{N}} \overline{T_{m}}$ é um subsemigrupo. Para isso usaremos a condição dada em 1.34. Sejam $m \in \mathbb{N}$ e $x_{n_{1}}+\cdots+x_{n_{k}} \in T_{m}$. Defina $m^{\prime}=m+\sum_{i=1}^{k} x_{n_{i}}$. Quero ver que $x_{n_{1}}+\cdots+x_{n_{k}}+T_{m^{\prime}} \subset$ $T_{m}$. Seja $x_{n_{j_{1}}}+\cdots+x_{n_{j_{l}}} \in T_{m^{\prime}}$. Como $x_{n_{j_{1}}}>m^{\prime}>x_{n_{k}}$, então $n_{j_{1}}>n_{k}$. Logo temos que $n_{1}<\ldots<n_{k}<n_{j_{1}}<\ldots<n_{j_{l}} ; x_{n_{1}}>m$; para $r \in\{1, \ldots, k\}, x_{n_{r}+1}-x_{n_{r}}>m+\sum_{i=1}^{r-1} x_{n_{i}}$; para $r \in\{1, \ldots, l\}, x_{n_{j_{r}+1}}-x_{n_{j_{r}}}>m^{\prime}+\sum_{i=1}^{r-1} x_{n_{j_{i}}}=m+\sum_{i=1}^{k} x_{n_{i}}+\sum_{i=1}^{r-1} x_{n_{j+i}}$. Destarte, $x_{n_{1}}+\cdots+x_{n_{k}}+x_{n_{j_{1}}}+\cdots+x_{n_{j_{l}}} \in T_{m}$, como queríamos.

Além disso, note no argumento acima que $c\left(x_{n_{1}}+\cdots+x_{n_{k}}+x_{n_{j_{1}}}+\cdots+x_{n_{j_{l}}}\right)=k+l=c\left(x_{n_{1}}+\cdots+\right.$ $\left.x_{n_{k}}\right)+c\left(x_{n_{j_{1}}}+\cdots+x_{n_{j_{l}}}\right)$, e portanto, pelo teorema 1.36, $\widetilde{c}$ é um homomorfismo sobre $\bigcap_{m \in \mathbb{N}} \overline{T_{m}}$. Como sabemos que $\overline{\left\{q_{n}: n \in \mathbb{N}\right\}} \subset \bigcap_{m \in \mathbb{N}} \overline{T_{m}}$, e por hipótese $\overline{\left\{q_{n}: n \in \mathbb{N}\right\}}$ é um subsemigrupo, podemos dizer que $\widetilde{c}$ é um homomorfismo sobre $\overline{\left\{q_{n}: n \in \mathbb{N}\right\}}$.

Considere, agora, $\phi: \mathbb{N} \rightarrow \mathbb{N}^{*}$ definida por $\phi(n)=q_{n}$. Note que $\phi[\mathbb{N}]=\left\{q_{n}: n \in \mathbb{N}\right\}$, $\operatorname{logo}$ $\widetilde{\phi}[\beta \mathbb{N}]=\overline{\left\{q_{n}: n \in \mathbb{N}\right\}}$. Além disso, $\widetilde{c}(\phi(n))=n$ e $\phi\left(\widetilde{c}\left(q_{n}\right)\right)=q_{n}$ para todo $n \in \mathbb{N}$, do que segue que $\widetilde{c}(\widetilde{\phi}(s))=s$ para todo $s \in \beta \mathbb{N}$ e $\widetilde{\phi}(\widetilde{c}(t))=t$ para todo $t \in \overline{\left\{q_{n}: n \in \mathbb{N}\right\}}$. Portanto, usando que $\widetilde{c}$ é um homomorfismo sobre $\overline{\left\{q_{n}: n \in \mathbb{N}\right\}}$ temos que, dados $s, r \in \beta \mathbb{N}, \widetilde{\phi}(s+r)=\widetilde{\phi}(\widetilde{c}(\widetilde{\phi}(s))+\widetilde{c}(\widetilde{\phi}(r)))=$ $\widetilde{\phi}(\widetilde{c}(\widetilde{\phi}(s)+\widetilde{\phi}(r)))=\widetilde{\phi}(s)+\widetilde{\phi}(r)$. Ou seja, $\widetilde{\phi}: \beta \mathbb{N} \rightarrow \mathbb{N}^{*}$ é um homomorfismo contínuo; para contradizer o Teorema 2.35, basta ver que a imagem é infinita. De fato, $\left\{q_{n}: n \in \mathbb{N}\right\}$ é infinito; caso $q_{k}=q_{n} \operatorname{com} n>k$, então $1+q_{k}=1+q_{n}$. Cancelando $q k$ vezes à direita, obtemos $1=1+q_{n-k}$, impossível.

Em contraste, o Teorema 4.4 de [3] nos dá uma classe de exemplos em que $\overline{\left\{q_{n}: n \in \mathbb{N}\right\}}$ é um 
subsemigrupo.

Teorema 2.44. Sejam $p$ um idempotente, $k \in \mathbb{N}$, e $q=k+p$. Então $\overline{\left\{q_{n}: n \in \mathbb{N}\right\}}$ é um subsemigrupo.

Demonstração. Usando que $\mathbb{N}$ é o centro de $\beta \mathbb{N}$, temos que $\left\{q_{n}: n \in \mathbb{N}\right\}=k \mathbb{N}+p, \operatorname{logo}$ $\overline{\left\{q_{n}: n \in \mathbb{N}\right\}}=\overline{k \mathbb{N}+p}=\overline{k \mathbb{N}}+p$. Note pelo teorema 1.34 que $\overline{k \mathbb{N}}$ é um subsemigrupo, e pelo lema 2.2 que $p \in \overline{k \mathbb{N}}$, logo dados $r_{1}, r_{2} \in \overline{k \mathbb{N}},\left(r_{1}+p\right)+\left(r_{2}+p\right)=\left(r_{1}+p+r_{2}\right)+p \in \overline{k \mathbb{N}}+p$. Portanto $\overline{k \mathbb{N}}+p$ é um subsemigrupo. 


\section{$2.4 \quad$ Subgrupos discretos em $\beta \mathbb{N}$}

Esta seção se devotará ao estudo do artigo "Discrete Groups in $\beta \mathbb{N}$ ", de Dona Strauss e Neil Hindman ([11]). Os primeiros dois resultados mostrarão que existem $2^{\mathfrak{c}}$ cópias discretas de $\mathbb{Z}$ em cada grupo maximal de $K(\beta \mathbb{N})$, cada par se intersectando somente na identidade. Lembramos que pelo Teorema 1.16, um conjunto é um grupo maximal em $K(\beta \mathbb{N})$ se e somente se é da forma $q+\beta \mathbb{N}+q, \operatorname{com} q \in K(\beta \mathbb{N})$ idempotente (minimal).

Novamente, usaremos a notação $\operatorname{supp}(x) \in \mathcal{P}_{f}(\omega)$ definida para $x \in \mathbb{N}$ como a representação binária de $x$, ou seja, $x=\sum_{t \in \operatorname{supp}(x)} 2^{t}$. Recordamos também o Lema 2.2, que será usado a seguir, e o subsemigrupo $\mathbb{H}=\bigcap_{m \geq 1} \overline{2^{m} \mathbb{N}}$.

Teorema 2.45. Sejam $A, B \subset \mathbb{N}$ infinitos e disjuntos, $q \in K(\beta \mathbb{N})$ idempotente, $u \in\left\{2^{n}: n \in A\right\}^{*}$, $v \in\left\{2^{n}: n \in B\right\}^{*}$. Considere $\varphi, \psi: \mathbb{Z} \rightarrow q+\beta \mathbb{N}+q$ os homomorfismos determinados por $\varphi(1)=q+u+q$ e $\psi(1)=q+v+q . \quad$ Então $\overline{\{\varphi(n): n \in \mathbb{Z} \backslash\{0\}\}} \cap \overline{\{\psi(n): n \in \mathbb{Z} \backslash\{0\}\}}=\varnothing$. Além disso, se $q \notin \overline{\{\varphi(n): n \in \mathbb{Z} \backslash\{0\}\}}$, então $\{\varphi(n): n \in \mathbb{Z}\}$ é uma cópia discreta de $\mathbb{Z}$; e se $q \notin \overline{\{\psi(n): n \in \mathbb{Z} \backslash\{0\}\}}$, então $\{\psi(n): n \in \mathbb{Z}\}$ é uma cópia discreta de $\mathbb{Z}$.

Demonstração. Primeiramente, note que $q \in \mathbb{H}$; e como $A$ e $B$ são infinitos e $u, v \in \mathbb{N}^{*}, u, v \in \mathbb{H}$. Segue que $\varphi[\mathbb{Z}] \cup \psi[\mathbb{Z}] \subset \mathbb{H}$. Note também que $\varphi(0)=\psi(0)=q$.

Agora, mostraremos que para quaisquer $m \in \mathbb{Z}$ e $n \in \mathbb{N}$,

$\{x \in \mathbb{N}:|\operatorname{supp}(x) \cap A| \equiv 0(\bmod n)\} \in \psi(m), \mathrm{e}$

$\{x \in \mathbb{N}:|\operatorname{supp}(x) \cap B| \equiv 0(\bmod n)\} \in \varphi(m)$.

Por analogia, basta mostrar a primeira asserção. Fixe $n \in \mathbb{N}$. Defina $C=\{x \in \mathbb{N}:|\operatorname{supp}(x) \cap A| \equiv 0$ $(\bmod n)\}$. Mostraremos que $C \in q$. Tome $i \in\{0, \ldots, n-1\}$ tal que $D:=\{x \in \mathbb{N}:|\operatorname{supp}(x) \cap A| \equiv i$ $(\bmod n)\} \in q . \quad$ Temos $\{x \in \mathbb{N}:-x+D \in q\} \in q$, logo tome $x \in D$ tal que $-x+D \in q$. Seja $t=\max \operatorname{supp}(x)$ e tome $y \in(-x+D) \cap D \cap 2^{t+1} \mathbb{N}(\in q)$. Note que $x+y \in D$ e que $\operatorname{supp}(x+y)=\operatorname{supp}(x) \cup \operatorname{supp}(y)$ e $\operatorname{supp}(x) \cap \operatorname{supp}(y)=\varnothing$. Portanto, $i \equiv|\operatorname{supp}(x+y) \cap A|=$ $|\operatorname{supp}(x) \cap A|+|\operatorname{supp}(y) \cap A| \equiv i+i(\bmod n)$, de forma que $i=0$. Ou seja, $D=C$.

Agora, por indução em $m \in \omega$ mostraremos que $C \in \psi(m)$. Acima mostramos que $C \in \psi(0)$. Suponha que $C \in \psi(m)$. Observe que $\psi(m+1)=\psi(m)+\psi(1)=\psi(m)+q+v+q=\psi(m)+v+q$. Para ver que $C \in \psi(m)+v+q$, basta ver que $C \subset\{x \in \mathbb{N}:-x+C \in v+q\}$, logo seja $x \in C$. Seja $t=\max \operatorname{supp}(x)$. Como $\left\{2^{s}: s \in B\right.$ e $\left.s>t\right\} \in v$, para ver que $-x+C \in v+q$ basta ver que $\left\{2^{s}: s \in B\right.$ e $\left.s>t\right\} \subset\{z \in \mathbb{N}:-z+(-x+C) \in q\}$. Seja $s \in B$ tal que $s>t$. Como $C \cap 2^{s+1} \mathbb{N} \in q$, para ver que $-2^{s}+(-x+C) \in q$ basta ver que $C \cap 2^{s+1} \mathbb{N} \subset-2^{s}+(-x+C)$. Seja então $y \in C \cap 2^{s+1} \mathbb{N}$. Como $s \notin A$, vale que $\left|\operatorname{supp}\left(x+2^{s}+y\right) \cap A\right|=|\operatorname{supp}(x) \cap A|+|\operatorname{supp}(y) \cap A| \equiv 0$ $(\bmod n)$, como queríamos.

Para completar esta parte, mostraremos que dado $m \in \mathbb{N}, C \in \psi(-m)$. Tome $i \in\{0, \ldots, n-1\}$ 
tal que $D=\{x \in \mathbb{N}:|\operatorname{supp}(x) \cap A| \equiv i(\bmod n)\} \in \psi(-m)$. Vale que $C \in q=\psi(-m)+\psi(m)$, ou seja $\{x \in \mathbb{N}:-x+C \in \psi(m)\} \in \psi(-m)$, logo tome $x \in D$ tal que $-x+C \in \psi(m)$. Seja $t=\max \operatorname{supp}(x)$ e tome $y \in(-x+C) \cap C \cap 2^{t+1} \mathbb{N}(\in \psi(m))$. Segue que $0 \equiv|\operatorname{supp}(x+y) \cap A|=$ $|\operatorname{supp}(x) \cap A|+|\operatorname{supp}(y) \cap A| \equiv i+0$, de forma que $i=0$. Ou seja, $D=C$.

Será também necessário mostrar que para quaisquer $m \in \mathbb{Z}$ e $n \in \mathbb{N}$,

$\{x \in \mathbb{N}:|\operatorname{supp}(x) \cap A| \equiv m(\bmod n)\} \in \varphi(m), \mathrm{e}$

$\{x \in \mathbb{N}:|\operatorname{supp}(x) \cap B| \equiv m(\bmod n)\} \in \psi(m)$.

Novamente as provas das duas asserções são análogas, logo faremos a primeira. Fixe $n \in \mathbb{N}$ e defina, para cada $m \in \mathbb{Z}, C_{m}=\{x \in \mathbb{N}:|\operatorname{supp}(x) \cap A| \equiv m(\bmod n)\}$. Nosso objetivo é mostrar que $C_{m} \in \varphi(m)$ para todo $m \in \mathbb{Z}$. Note que já foi mostrado acima que $C_{0}=C \in q=\varphi(0)$. Faremos novamente uma indução em $m \in \omega$. Suponha que $C_{m} \in \varphi(m)$. Para ver que $C_{m+1} \in \varphi(m+1)=$ $\varphi(m)+u+q$, basta ver que $C_{m} \subset\left\{x \in \mathbb{N}:-x+C_{m+1} \in u+q\right\}$, logo seja $x \in C_{m}$. Seja $t=\max \operatorname{supp}(x)$. Para ver que $-x+C_{m+1} \in u+q$, como $\left\{2^{s}: s \in A\right.$ e $\left.s>t\right\} \in u$, basta ver que $\left\{2^{s}: s \in A\right.$ e $\left.s>t\right\} \subset\left\{z \in \mathbb{N}:-z+\left(-x+C_{m+1}\right) \in q\right\}$. Seja $s \in A \operatorname{com} s>t$. Como $C_{0} \cap 2^{s+1} \mathbb{N} \in q$, para ver que $-2^{s}+\left(-x+C_{m+1}\right) \in q$ basta ver que $C_{0} \cap 2^{s+1} \mathbb{N} \subset-2^{s}+\left(-x+C_{m+1}\right)$. Seja então $y \in C_{0} \cap 2^{s+1} \mathbb{N}$. Vale que $\left|\operatorname{supp}\left(x+2^{s}+y\right) \cap A\right|=|\operatorname{supp}(x) \cap A|+1+|\operatorname{supp}(y) \cap A| \equiv m+1+0$ $(\bmod n)$, como queríamos.

Para completar, seja $m \in \mathbb{N}$ e vejamos que $C_{-m} \in \varphi(-m)$. Seja $i \in\{0, \ldots, n-1\}$ tal que $D=\{x \in \mathbb{N}:|\operatorname{supp}(x) \cap A| \equiv i(\bmod n)\} \in \varphi(-m)$. Temos que $C_{0} \in q=\varphi(-m)+\varphi(m)$, ou seja $\left\{x \in \mathbb{N}:-x+C_{0} \in \varphi(m)\right\} \in \varphi(-m)$, logo tome $x \in D$ tal que $-x+C_{0} \in \varphi(m)$. Seja $t=\max \operatorname{supp}(x)$ e tome $y \in\left(-x+C_{0}\right) \cap C_{m} \cap 2^{t+1} \mathbb{N}(\in \varphi(m))$. Vale que $0 \equiv|\operatorname{supp}(x+y) \cap A|=$ $|\operatorname{supp}(x) \cap A|+|\operatorname{supp}(y) \cap A| \equiv i+m(\bmod n)$, de forma que $i \equiv-m(\bmod n)$ e assim $D=C$ como queríamos.

Note que as asserções provadas acima garantem que se $k, m \in \mathbb{Z}$ são distintos, então $\varphi(k) \neq \varphi(m)$ e $\psi(k) \neq \psi(m)$, e portanto $\varphi[\mathbb{Z}]$ e $\psi[\mathbb{Z}]$ são cópias algébricas de $\mathbb{Z}$.

Então, suponha que $\overline{\{\varphi(n): n \in \mathbb{Z} \backslash\{0\}\}} \cap \overline{\{\psi(n): n \in \mathbb{Z} \backslash\{0\}\}} \neq \varnothing$. Pelo Teorema 1.26(c), temos, sem perda de generalidade, que $\{\varphi(n): n \in \mathbb{Z} \backslash\{0\}\} \cap \overline{\{\psi(n): n \in \mathbb{Z} \backslash\{0\}\}} \neq \varnothing$; logo tome $m \in \mathbb{Z} \backslash\{0\}$ tal que $\varphi(m) \in \overline{\{\psi(n): n \in \mathbb{Z} \backslash\{0\}\}}$. Porém, $\overline{\{x \in \mathbb{N}:|\operatorname{supp}(x) \cap A| \equiv m(\bmod |m|+1)\}}$ é uma vizinhança de $\varphi(m)$ disjunta de $\overline{\{x \in \mathbb{N}:|\operatorname{supp}(x) \cap A| \equiv 0(\bmod |m|+1)\}}$, e $\{\psi(n): n \in \mathbb{Z}\} \subset \overline{\{x \in \mathbb{N}:|\operatorname{supp}(x) \cap A| \equiv 0(\bmod |m|+1)\}}$; uma contradição.

Por fim, suponha que $q \notin \overline{\{\varphi(n): n \in \mathbb{Z} \backslash\{0\}\}}$. Tome $U$ vizinhança de $q$ disjunta de $\{\varphi(n)$ : $n \in \mathbb{Z} \backslash\{0\}\}$. Dado $n \in \mathbb{Z} \backslash\{0\}, U$ é uma vizinhança de $\varphi(n)+\varphi(-n)=\rho_{\varphi(-n)}(\varphi(n))$, logo seja $V$ vizinhança de $\varphi(n)$ tal que $\rho_{\varphi(-n)}[V]=V+\varphi(-n) \subset U$. Como $U \cap\{\varphi(n): n \in \mathbb{Z} \backslash\{0\}\}=\varnothing$, segue que $V \cap\{\varphi(m): m \in \mathbb{Z} \backslash\{n\}\}=\varnothing$. Assim, $\{\varphi(n): n \in \mathbb{Z}\}$ é discreto.

Em resumo, temos o seguinte corolário: 
Corolário 2.46. Seja $q \in K(\beta \mathbb{N})$ idempotente. Para cada $p \in\left\{2^{n}: n \in \mathbb{N}\right\}^{*}$, seja $\varphi_{p}: \mathbb{Z} \rightarrow$ $q+\beta \mathbb{N}+q$ o homomorfismo determinado por $\varphi_{p}(1)=q+p+q$. Então, dados $p, r \in\left\{2^{n}: n \in \mathbb{N}\right\}^{*}$ distintos, $\overline{\left\{\varphi_{p}(n): n \in \mathbb{Z} \backslash\{0\}\right\}} \cap \overline{\left\{\varphi_{r}(n): n \in \mathbb{Z} \backslash\{0\}\right\}}=\varnothing$. Além disso, existe no máximo um $p \in \mathbb{N}^{*}$ tal que $\left\{\varphi_{p}(n): n \in \mathbb{Z}\right\}$ não é discreto.

Demonstração. Para a primeira afirmação, como $p$ e $r$ são distintos, existem $A, B \subset \mathbb{N}$ disjuntos tais que $\left\{2^{n}: n \in A\right\} \in p$ e $\left\{2^{n}: n \in B\right\} \in r$, de forma que podemos aplicar o Teorema 2.45. Ademais, suponha que $p, p^{\prime} \in \mathbb{N}^{*}$ são tais que $\left\{\varphi_{p}(n): n \in \mathbb{Z}\right\}$ e $\left\{\varphi_{p^{\prime}}(n): n \in \mathbb{Z}\right\}$ não são discretos. Então $q \in \overline{\left\{\varphi_{p}(n): n \in \mathbb{Z} \backslash\{0\}\right\}} \cap \overline{\left\{\varphi_{p^{\prime}}(n): n \in \mathbb{Z} \backslash\{0\}\right\}}$. Pelo que acabamos de ver acima, isso implica que $p=p^{\prime}$.

O restante do artigo trata da existência de cópias de grupos e semigrupos livres dentro dos grupos maximais de $K(\beta \mathbb{N})$. Primeiramente, estabeleceremos a existência de $2^{\mathfrak{c}}$ cópias do grupo livre sobre 2 geradores, disjuntas exceto pela identidade.

Lema 2.47. Existe um grupo topológico compacto $C$ que contém um grupo livre $F$ sobre os geradores distintos $\left\{a_{1}, a_{2}, a_{3}, a_{4}\right\}$.

Demonstração. Isso é um caso particular de 3.16.

Lema 2.48. Sejam $C$ e $F$ como no Lema 2.4\%. Sejam $A_{1}, A_{2}, A_{3}, A_{4} \subset \mathbb{N}$ dois-a-dois disjuntos e seja $q \in K(\beta \mathbb{N})$ idempotente. Para $i \in\{1,2,3,4\}$ seja $u_{i} \in\left\{2^{n}: n \in A_{i}\right\}^{*}$ qualquer e defina $r_{i}=q+u_{i}+q$. Seja $G$ o subgrupo de $q+\beta \mathbb{N}+q$ gerado por $\left\{r_{1}, r_{2}, r_{3}, r_{4}\right\}$. Então existe um homomorfismo contínuo $\sigma:\{0\} \cup \mathbb{H} \rightarrow C$ tal que $\left.\sigma\right|_{G}$ é um isomorfismo entre $G$ e $F$ e $\sigma\left(r_{i}\right)=a_{i}$ para cada $i \in\{1,2,3,4\}$.

Demonstração. Denote a identidade de $C$ por e. Defina $f: \omega \rightarrow C$ assim: $f(0)=e$. Dado $n \in \omega, f\left(2^{n}\right)=a_{i}$ se $n \in A_{i} ; f\left(2^{n}\right)=e$ caso $n \notin A_{1} \cup A_{2} \cup A_{3} \cup A_{4}$. E dado $I \in \mathcal{P}_{f}(\omega)$, $f\left(\sum_{n \in I} 2^{n}\right)=\prod_{n \in I} f\left(2^{n}\right)$. Tome $\tilde{f}: \beta \omega \rightarrow C$ a extensão contínua de $f$, e seja $\sigma$ a restrição de $\widetilde{f}$ a $\{0\} \cup \mathbb{H}$. Como $\{0\} \cup \mathbb{H}=\bigcap_{n \in \mathbb{N}} 2^{n} \omega$, podemos aplicar o Teorema 1.36 sobre a coleção $\mathcal{A}=\left\{2^{n} \omega: n \in \mathbb{N}\right\}$ e obter que $\sigma$ é um homomorfismo.

Para ver que $\sigma[G]=F$, basta ver que $\sigma\left(r_{i}\right)=a_{i}$ para cada $i \in\{1,2,3,4\}$. Note que $\sigma$ é constante e igual a $a_{i}$ sobre $\left\{2^{n}: n \in A_{i}\right\}$, de forma que $\sigma\left(u_{i}\right)=a_{i}$. E como $q$ é idempotente, $\sigma(q)=e$. Assim $\sigma\left(r_{i}\right)=e a_{i} e=a_{i}$.

E para ver que $\sigma$ é injetora, considere $h: F \rightarrow G$ o homomorfismo determinado por $h\left(a_{i}\right)=r_{i}$ para cada $i \in\{1,2,3,4\}$. Temos que $\sigma \circ h\left(a_{i}\right)=r_{i}$ para cada $i \in\{1,2,3,4\}$, de forma que $\sigma \circ h: F \rightarrow F$ é a identidade, e portanto $\sigma$ é injetora. 
Teorema 2.49. Sejam $A_{1}, A_{2}, A_{3}, A_{4}, F, C, u_{1}, u_{2}, u_{3}, u_{4}, r_{1}, r_{2}, r_{3}, r_{4}, G$ e $\sigma$ como no Lema acima. Sejam $G_{1}$ o subgrupo de $G$ gerado por $\left\{r_{1}, r_{2}\right\}$ e $G_{2}$ o subgrupo gerado por $\left\{r_{3}, r_{4}\right\}$. Então $\overline{G_{1} \backslash\{q\}} \cap \overline{G_{2} \backslash\{q\}}=\varnothing$. Além disso, dado $i\{1,2\}$, se $q \notin \overline{G_{i} \backslash\{q\}}$, então $G_{i}$ é discreto.

Demonstração. Suponha que $\overline{G_{1} \backslash\{q\}} \cap \overline{G_{2} \backslash\{q\}} \neq \varnothing$. Pelo Teorema 1.26(c), sem perda de generalidade, tome $w \in G_{1} \backslash\{q\} \cap \overline{G_{2} \backslash\{q\}}$. Mostraremos, contraditoriamente, que $w=q$. Denote por $s_{1}$ e $s_{2}$ as inversas de $r_{1}$ e $r_{2}$ em $G_{1}$, respectivamente. Tome $p_{1}, \ldots, p_{m} \in\left\{r_{1}, r_{2}, s_{1}, s_{2}\right\}$ tais que $w=p_{1}+\cdots+p_{m}$.

Defina $\theta: \mathbb{N} \rightarrow \omega$ por $\theta(n)=\sum\left\{2^{t}: t \in \operatorname{supp}(n) \cap\left(A_{1} \cup A_{2}\right)\right\}$ e seja $\widetilde{\theta}: \beta \mathbb{N} \rightarrow \beta \omega$ sua extensão contínua. Usando o Teorema 1.36, vê-se que $\left.\widetilde{\theta}\right|_{\mathbb{H}}$ é um homomorfismo. Além disso, note que dados $n, m \in \mathbb{N}, \theta\left(2^{m} n\right) \in 2^{m} \mathbb{N}$, de forma que usando $\mathbb{H}=\bigcap_{m \in \mathbb{N}} \overline{2^{m} \mathbb{N}}$ e o Teorema $1.26(\mathrm{e})$, temos que $\widetilde{\theta}[\mathbb{H}] \subset \mathbb{H} \cup\{0\}=\operatorname{dom} \sigma$.

Para $i \in\{1,2\}, \theta$ é a identidade sobre $\left\{2^{n}: n \in A_{i}\right\}$, de modo que $\widetilde{\theta}\left(u_{i}\right)=u_{i}$; e $\sigma(\widetilde{\theta}(q))=e$ pois $q$ é idempotente. Temos então: $\sigma\left(\widetilde{\theta}\left(r_{i}\right)\right)=\sigma\left(\widetilde{\theta}\left(q+u_{i}+q\right)\right)=e \sigma\left(\widetilde{\theta}\left(u_{i}\right)\right) e=e \sigma\left(u_{i}\right) e=\sigma\left(q+u_{i}+q\right)=$ $\sigma\left(r_{i}\right) . \operatorname{E} \sigma\left(\widetilde{\theta}\left(s_{i}\right)\right)=\sigma\left(\widetilde{\theta}\left(r_{i}\right)\right)^{-1}=\sigma\left(r_{i}\right)^{-1}=\sigma\left(s_{i}\right)$.

Por outro lado, para $i \in\{3,4\}, \theta$ é nula sobre $\left\{2^{n}: n \in A_{i}\right\}$, de forma que $\widetilde{\theta}\left(u_{i}\right)=0$, e assim $\sigma\left(\widetilde{\theta}\left(r_{i}\right)\right)=\sigma\left(\widetilde{\theta}\left(\left(q+u_{i}+q\right)\right)=e e e=e\right.$. Disso segue que $\sigma \circ \widetilde{\theta}\left[G_{2}\right]=\{e\}$. Portanto, por continuidade, como $w \in \overline{G_{2}}, \sigma(\widetilde{\theta}(w))=e$. Temos então que $\sigma(q)=e=\sigma(\widetilde{\theta}(w))=\sigma\left(\widetilde{\theta}\left(\left(p_{1}+\cdots+p_{m}\right)\right)=\right.$ $\sigma\left(p_{1}+\cdots+p_{m}\right)=\sigma(w)$. Porém, como vimos no Lema 2.48, $\sigma$ é um isomorfismo sobre $G$, de forma que $w=q$.

Por fim, seja $i \in\{1,2\}$ e suponha que $q \notin \overline{G_{i} \backslash\{q\}}$. Tome $U$ vizinhança de $q$ disjunta de $G_{i} \backslash\{q\}$. Seja $w \in G_{i} \backslash\{q\}$ qualquer, e seja $w^{\prime}$ sua inversa neste grupo; temos que $U$ é uma vizinhança de $w+w^{\prime}$, logo seja $V$ vizinhança de $w$ tal que $V+w^{\prime} \subset U$. Então $V \cap G_{i} \backslash\{w\}=\varnothing$, e assim, $G_{i}$ é discreto.

Corolário 2.50. Seja $q \in K(\beta \mathbb{N})$ um idempotente. Então existem $2^{\mathfrak{c}}$ cópias discretas do grupo livre sobre 2 geradores em $(q+\beta \mathbb{N}+q) \cap \mathbb{H}$. A interseção dos fechos de quaisquer dois grupos desse tipo $\dot{e}\{q\}$.

Demonstração. Particione $\left\{2^{n}: n \in \mathbb{N}\right\}^{*}$ em $2^{\mathfrak{c}}$ conjuntos de dois elementos: $\left\{x_{\alpha}, y_{\alpha}\right\}$ para $\alpha<2^{\mathfrak{c}}$. Para cada $\alpha$, seja $G_{\alpha}$ o subgrupo de $q+\beta \mathbb{N}+q$ gerado por $\left\{q+x_{\alpha}+q, q+y_{\alpha}+q\right\}$. Então se $\alpha<\beta<2^{\mathfrak{c}}$, podemos tomar $A_{1}, A_{2}, A_{3}, A_{4} \subset \mathbb{N}$ disjuntos tais que $\left\{2^{n}: n \in A_{1}\right\} \in x_{\alpha},\left\{2^{n}: n \in A_{2}\right\} \in y_{\alpha}$, $\left\{2^{n}: n \in A_{3}\right\} \in x_{\beta}$ e $\left\{2^{n}: n \in A_{4}\right\} \in y_{\beta}$, de forma que o Teorema 2.49 se aplica, e temos que cada $G_{\alpha}$ é um grupo livre sobre dois geradores, e existe no máximo um deles que não é discreto.

Definição 2.51. Seja $X$ um espaço topológico. Dizemos que $Y \subset X$ é fortemente discreto se para cada $x \in Y$ existir $U_{x}$ vizinhança de $x$ em $X$ de forma que para todos $x, y \in Y$ distintos, 
$U_{x} \cap U_{y}=\varnothing$.

Teorema 2.52. Existe uma cópia fortemente discreta do semigrupo livre com identidade sobre $\mathfrak{c}$ geradores em $\mathbb{H}$. (Em particular é discreto em $\mathbb{N}^{*}$.)

Demonstração. Primeiramente, tome $\left\{A_{\alpha}: \alpha<\mathfrak{c}\right\}$ uma família quase disjunta de subconjuntos de $2 \mathbb{N}+1$, e tome $p_{\alpha} \in\left\{2^{n}: n \in A_{\alpha}\right\}^{*}$ para cada $\alpha<\mathfrak{c}$. Agora, precisamos tomar $q \in \overline{\{x \in \mathbb{N}: \operatorname{supp}(x) \subset 2 \mathbb{N}\}}$ idempotente. Para tanto, defina $C=\{x \in \mathbb{N}: \operatorname{supp}(x) \subset 2 \mathbb{N}\}$, e mostraremos que $\mathbb{H} \cap \bar{C}$ é um subsemigrupo (evidentemente compacto) de $\beta \mathbb{N}$ : defina, para cada $m \in \mathbb{N}$, $T_{m}=2^{m} \mathbb{N} \cap C$; vale que $\mathbb{H} \cap \bar{C}=\bigcap_{m \in \mathbb{N}} \overline{T_{m}}$, e dados $m \in \mathbb{N}$ e $x \in T_{m}$, tome $n=\max \operatorname{supp}(x)+1 ;$ dado $y \in T_{n}$, vale que $\operatorname{supp}(x+y)=\operatorname{supp}(x) \cup \operatorname{supp}(y) \subset 2 \mathbb{N}$ e $x+y \in T_{m} ;$ assim, aplica-se o Teorema 1.34 e temos que $\mathbb{H} \cap \bar{C}$ é um subsemigrupo compacto, logo possui um idempotente $q$.

Defina agora, para cada $\alpha<\mathfrak{c}, r_{\alpha}=q+p_{\alpha}+q$. Observe que $S=\left\{r_{\alpha}: \alpha<\mathfrak{c}\right\} \subset \mathbb{H}$, pois $\mathbb{H}$ é subsemigrupo, $q \in \mathbb{H}$ e $p_{\alpha} \in \mathbb{H}$ para cada $\alpha<\mathfrak{c}$. E como $q+q=q, q$ é uma identidade para $S$. Veremos que $S$ gera um semigrupo livre com identidade $q$ que é fortemente discreto em $\mathbb{H}$.

Para cada $\left(\alpha_{1}, \ldots, \alpha_{k}\right)$ sequência finita em $\mathfrak{c}$, considere $B_{\left(\alpha_{1}, \ldots, \alpha_{k}\right)}=\{x \in \mathbb{N}: \operatorname{supp}(x) \cap(2 \mathbb{N}+1)=$ $\left\{n_{1}, \ldots, n_{k}\right\}$ em que $n_{1}<\cdots<n_{k}$ e $n_{i} \in A_{\alpha_{i}}$ para cada $\left.i \in\{1, \ldots, k\}\right\}$.

Afirmação 1: Para cada $\alpha<\mathfrak{c}, B_{(\alpha)} \in r_{\alpha}$. Lembre que $C \in q$. Para cada $x \in C$, defina $D_{x}=\left\{2^{n}: n \in A_{\alpha}\right.$ e $\left.n>\max \operatorname{supp}(x)\right\}$ e note que $D_{x} \in p_{\alpha}$. Para cada $x \in C$ e cada $n \in A_{\alpha}$ com $n>\max \operatorname{supp}(x)$, defina $E_{x, n}=\{z \in C: \min \operatorname{supp}(z)>n\}$ e note que $E_{x, n} \in q$. Logo podemos aplicar o Lema 2.7 e obter que $P=\left\{x+2^{n}+z: x \in C, n \in A_{\alpha}\right.$ e $\left.\max \operatorname{supp}(x)<n<\min \operatorname{supp}(z)\right\} \in$ $q+p_{\alpha}+q=r_{\alpha}$. Agora, dado $x+2^{n}+z \in P, \operatorname{supp}\left(x+2^{n}+z\right)=\operatorname{supp}(x) \cup\{n\} \cup \operatorname{supp}(z) ; \operatorname{como}$ $x, z \in C$, segue que $\operatorname{supp}\left(x+2^{n}+z\right) \cap(2 \mathbb{N}+1)=\{n\}$, e $n \in A_{\alpha}$. Segue que $P \subset B_{(\alpha)}$, $\log$ o $B_{(\alpha)} \in r_{\alpha}$.

Afirmação 2: Dados $k>1$, e $\left(\alpha_{1}, \ldots, \alpha_{k}\right) \in \mathfrak{c}^{<\omega}$, então $B_{\left(\alpha_{1}, \ldots, \alpha_{k-1}\right)} \subset\left\{x \in \mathbb{N}:-x+B_{\left(\alpha_{1}, \ldots, \alpha_{k}\right)} \in\right.$ $\left.r_{\alpha_{k}}\right\}$. Seja então $x \in B_{\left(\alpha_{1}, \ldots, \alpha_{k-1}\right)}$. Quero ver que $-x+B_{\left(\alpha_{1}, \ldots, \alpha_{k}\right)} \in r_{\alpha_{k}}$. Como $r_{\alpha_{k}} \in \mathbb{H}$, tome $n=\max \operatorname{supp}(x)+1$; basta ver então que $B_{\left(\alpha_{k}\right)} \cap 2^{n} \mathbb{N} \subset-x+B\left(\alpha_{1}, \ldots, \alpha_{k-1}\right)$; para isso, seja $y \in B_{\left(\alpha_{k}\right)} \cap 2^{n} \mathbb{N}$, e tome para cada $i \in\{1, \ldots, k\}, n_{i} \in A_{\alpha_{i}}$ de forma que $\operatorname{supp}(x) \cap(2 \mathbb{N}+1)=$ $\left\{n_{1}, \ldots, n_{k-1}\right\}$ com $n_{1}<\cdots<n_{k-1}$ e $\operatorname{supp}(y) \cap(2 \mathbb{N}+1)=\left\{n_{k}\right\}$. Temos então que $n_{k}>n_{k-1}$, e que $\operatorname{supp}(x+y) \cap(2 \mathbb{N}+1)=(\operatorname{supp}(x) \cap(2 \mathbb{N}+1)) \cup(\operatorname{supp}(y) \cap(2 \mathbb{N}+1))=\left\{n_{1}, \ldots, n_{k}\right\}$, e assim $x+y \in B_{\left(\alpha_{1}, \ldots, \alpha_{k}\right)}$.

Afirmação 3: Dados $k>1$ e $\left(\alpha_{1}, \ldots, \alpha_{k}\right) \in \mathfrak{c}^{<\omega}, B_{\left(\alpha_{1}, \ldots, \alpha_{k}\right)} \in r_{\alpha_{1}}+\cdots+r_{\alpha_{k}}$. Por indução: vimos acima que $B_{\left(\alpha_{1}\right.} \in r_{\alpha_{1}}$ e $B_{\left(\alpha_{1}\right)} \subset\left\{x \in \mathbb{N}:-x+B_{\left(\alpha_{1}, \alpha_{2}\right)} \in r_{\alpha_{2}}\right\}$, logo $B_{\left(\alpha_{1}, \alpha_{2}\right)} \in r_{\alpha_{1}}+r_{\alpha_{2}}$. Agora suponha que $B_{\left(\alpha_{1}, \ldots, \alpha_{k-1}\right)} \in r_{\alpha_{1}}+\cdots+r_{\alpha_{k-1}}$; como $B_{\left(\alpha_{1}, \ldots, \alpha_{k-1}\right)} \subset\left\{x \in \mathbb{N}:-x+B_{\left(\alpha_{1}, \ldots, \alpha_{k}\right)} \in r_{\alpha_{k}}\right\}$, segue que $B_{\left(\alpha_{1}, \ldots, \alpha_{k}\right)} \in r_{\alpha_{1}}+\cdots+r_{\alpha_{k}}$.

Assim, para ver que $S$ gera um semigrupo livre com identidade $q$, fortemente discreto em $\mathbb{H}$, note 
em primeiro lugar que, para cada $\left(\alpha_{1}, \ldots, \alpha_{k}\right) \in \mathfrak{c}^{<\omega} \backslash\{\varnothing\}, C \cap B_{\left(\alpha_{1}, \ldots, \alpha_{k}\right)}=\varnothing$. Em segundo lugar, vejamos que dados $\left(\alpha_{1}, \ldots, \alpha_{k}\right),\left(\delta_{1}, \ldots, \delta_{l}\right) \in \mathfrak{c}^{<\omega} \backslash\{\varnothing\}$ distintos, $\overline{B_{\left(\alpha_{1}, \ldots, \alpha_{k}\right)}} \cap \overline{B_{\left(\delta_{1}, \ldots, \delta_{l}\right)}} \cap \mathbb{H}=\varnothing$. Para tanto, observe em primeiro lugar que dado $x \in B_{\left(\alpha_{1}, \ldots, \alpha_{k}\right)},|\operatorname{supp}(x) \cap(2 \mathbb{N}+1)|=k$, e dado $x \in B_{\left(\delta_{1}, \ldots, \delta_{l}\right)},|\operatorname{supp}(x) \cap(2 \mathbb{N}+1)|=l$. Logo se $k \neq l, B_{\left(\alpha_{1}, \ldots, \alpha_{k}\right)} \cap B_{\left(\delta_{1}, \ldots, \delta_{l}\right)}=\varnothing$; se $k=l$, tome $j \in\{1, \ldots, k\}$ tal que $\alpha_{j} \neq \delta_{j}$; e tome $m \in \mathbb{N}$ tal que $A_{\alpha_{j}} \cap A_{\delta_{j}} \subset\{1, \ldots, m\}$. Como $B_{\left(\alpha_{1}, \ldots, \alpha_{k}\right)} \cap B_{\left(\delta_{1}, \ldots, \delta_{k}\right)}=\left\{x \in \mathbb{N}: \operatorname{supp}(x) \cap(2 \mathbb{N}+1)=\left\{n_{1}, \ldots, n_{k}\right\}\right.$ em que $n_{1}<\cdots<$ $n_{k}$ e $n_{i} \in A_{\alpha_{i}} \cap A_{\delta_{i}}$ para cada $\left.i \in\{1, \ldots, k\}\right\}$, então há de se ter que $n_{j} \leq m$, e portanto $B_{\left(\alpha_{1}, \ldots, \alpha_{k}\right)} \cap B_{\left(\delta_{1}, \ldots, \delta_{k}\right)} \cap 2^{m+1} \mathbb{N}=\varnothing ; \operatorname{assim} \overline{B_{\left(\alpha_{1}, \ldots, \alpha_{k}\right)}} \cap \overline{B_{\left(\delta_{1}, \ldots, \delta_{k}\right)}} \cap \mathbb{H}=\varnothing$.

O teorema acima nos dá um subsemigrupo de tamanho $\mathfrak{c}$, discreto em $\mathbb{N}^{*}$. Não podemos afirmar que ele é fortemente discreto em $\mathbb{N}^{*}$, embora o seja em $\mathbb{H}$. Por outro lado, temos que o conjunto $\left\{p_{\alpha}: \alpha<\mathfrak{c}\right\}$ acima é fortemente discreto em $\mathbb{N}^{*}$ (embora não o seja em $\beta \mathbb{N}$, que claramente possui celularidade enumerável), mas não sabemos se gera um subsemigrupo livre. O artigo indaga então se seria possível existir um subsemigrupo livre fortemente discreto de tamanho $\mathfrak{c}$ em $\mathbb{N}^{*}$, e responde que não; de fato, o Teorema a seguir dá uma incisiva resposta negativa: é consequência imediata dele que não é possível haver um subsemigrupo fortemente discreto, não-enumerável, para o qual existe um elemento cancelável à direita.

Teorema 2.53. Não existem $p \in \mathbb{N}^{*}$ e $\left\{r_{\alpha}: \alpha<\omega_{1}\right\} \subset \mathbb{N}^{*}$ tais que $r_{\alpha}+p \neq r_{\delta}+p$ sempre que $\alpha<\delta<\omega_{1} e\left\{r_{\alpha}+p: \alpha<\omega_{1}\right\}$ é fortemente discreto em $\mathbb{N}^{*}$.

Demonstração. Suponha que existem, e para cada $\alpha<\omega_{1}$ tome $B_{\alpha} \subset \mathbb{N}$ tais que $B_{\alpha} \in r_{\alpha}+p$ e $B_{\alpha} \cap B_{\delta}$ é finito para cada $\alpha<\delta<\omega_{1}$. Segue que $C_{\alpha}=\left\{x \in \mathbb{N}:-x+B_{\alpha} \in p\right\} \in r_{\alpha}$ para cada $\alpha<\omega_{1}$; como não podem ser dois-a-dois disjuntos, tome $\alpha, \beta<\omega_{1}$ tais que $C_{\alpha} \cap C_{\beta} \neq \varnothing$ e tome $x \in C_{\alpha} \cap C_{\beta}$. Temos então que $\left(-x+B_{\alpha}\right) \cap\left(-x+B_{\beta}\right) \in p$, logo é infinito, do que segue que $B_{\alpha} \cap B_{\beta}$ é infinito. Absurdo. 


\subsection{Cadeias de idempotentes}

Nesta seção estudaremos os artigos "Chains of idempotents in $\beta N$ " [13] e "Ideals and commutativity in $\beta \mathbb{N}$ " [14], e veremos que todo idempotente não-minimal de $\beta \mathbb{N}$ pertence a uma cadeia decrescente de idempotentes, cada um maximal com respeito a ser menor do que seu antecessor. O mesmo vale para cadeias de ideais à esquerda semiprincipais.

Começaremos com o artigo "Ideals and commutativity in $\beta \mathbb{N}$ " e veremos que existem cadeias de ideais à esquerda de tipo $\omega_{1}$.

Antes de irmos ao artigo, precisaremos de um importante lema sobre os ideais à esquerda semiprincipais de $\beta \mathbb{N}$, e de certos fatos acerca de um semigrupo de $\mathbb{N}^{*}$.

Lema 2.54. Sejam $p, q \in \beta \mathbb{N}$ distintos. $S e(\beta \mathbb{N}+p) \cap(\beta \mathbb{N}+q) \neq \varnothing$, então $p \in \beta \mathbb{N}+q$ ou $q \in \beta \mathbb{N}+p$.

Demonstração. Como $\beta \mathbb{N}+p=\overline{\mathbb{N}+p}$ e $\beta \mathbb{N}+q=\overline{\mathbb{N}}+q$, então pelo Teorema 1.26(c), e sem perda

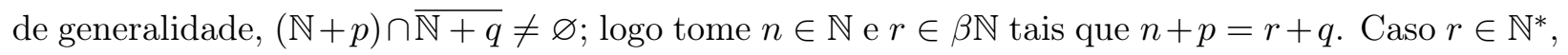
pelo Lema 2.11, $p=-n+r+q \in \beta \mathbb{N}+q$. Caso $r \in \mathbb{N}$, temos a tricotomia: se $r=n$, então $p=q$, absurdo; se $r<n$, então $q=-r+n+p \in \beta \mathbb{N}+p$; e se $r>n$, então $p=-n+r+q \in \beta \mathbb{N}+q$.

Nos seguintes teoremas, faremos uso do subsemigrupo de $\mathbb{N}^{*}, \bigcap_{k \in \mathbb{N}} \overline{k \mathbb{N}}$. Este subconjunto nãovazio, $G_{\delta}$ e compacto de $\beta \mathbb{N}$, que denotaremos por $\mathbb{T}$, é de fato um subsemigrupo: dado $k \in \mathbb{N}$ e dado $n \in k \mathbb{N}$, temos que $n+k \mathbb{N} \subset k \mathbb{N}$, satifazendo a condição do Teorema 1.34. Pelo Lema 2.2, temos que $\mathbb{T}$ possui todos os idempotentes.

Para cada $n \in \mathbb{N}$, denotaremos por $\gamma_{n}: \mathbb{Z} \rightarrow \mathbb{Z}_{n}$ o homomorfismo canônico, e portanto por $\widetilde{\gamma_{n}}$ sua extensão a $\beta \mathbb{Z}$. Dessa forma (lembrando que estamos usando a topologia discreta sobre o conjunto finito $\mathbb{Z}_{n}$ ), temos que $\mathbb{T}=\bigcap_{n \in \mathbb{N}}{\widetilde{\gamma_{n}}}^{-1}[\{\overline{0}\}]$.

Teorema 2.55. Seja $p \in \mathbb{N}^{*} \backslash K(\beta \mathbb{N})$. Então existe $G \subset \mathbb{T}$ aberto de $\mathbb{N}^{*}$ não-vazio tal que para todo $q \in G, p \notin \beta \mathbb{Z}+q+p$.

Demonstração. Para cada $Y \in p$ e cada $n \in \mathbb{Z}$, seja $K_{n, Y}=\{q \in \mathbb{T}: n+q+p \in \bar{Y}\}$. Note que $K_{n, Y}$ é um clopen de $\mathbb{T}$, pois é a imagem inversa, em $\mathbb{T}$, de $\bar{Y}$ pela função $q \mapsto n+q+p$, que é contínua.

Afirmamos que, para algum $Y \in p, \bigcup_{n \in \mathbb{Z}} K_{n, Y} \neq \mathbb{T}$. Suponha então que para todo $Y \in p$, $\bigcup_{n \in \mathbb{Z}} K_{n, Y}=\mathbb{T}$. Seja $q$ idempotente minimal; lembre que $q \in \mathbb{T}$. Logo, para cada $Y \in p$, tome $n_{Y} \in \mathbb{Z}$ e $p_{Y} \in \bar{Y}$ tais que $p_{Y}=n_{Y}+q+p$. Como $\left(n_{Y}\right)_{Y \in p}$ e $\left(p_{Y}\right)_{Y \in p}$ são redes em $\beta \mathbb{N}$ (direcionadas pela inclusão reversa), seja $r$ um ponto de acumulação de $\left(n_{Y}\right)_{Y \in p}$ e note que $\lim _{Y \in p} p_{Y}=p$. Assim, segue que $p=r+q+p$; como $q \in K(\beta \mathbb{N})$, tem-se que $p \in K(\beta \mathbb{N})$, uma contradição.

Logo, tome $Y \in p$ tal que $\bigcup_{n \in \mathbb{Z}} K_{n, Y} \neq \mathbb{T}$. Ou seja, $\mathbb{T} \backslash\left(\bigcup_{n \in \mathbb{Z}} K_{n, Y}\right)=\bigcap_{n \in \mathbb{Z}}\left(\mathbb{T} \backslash K_{n, Y}\right) \neq \varnothing$. Como $K_{n, Y}$ são clopens de $\mathbb{T}$ e $\mathbb{T}$ é $G_{\delta}$ de $\mathbb{N}^{*}$, então $\bigcap_{n \in \mathbb{Z}}\left(\mathbb{T} \backslash K_{n, Y}\right)$ é um $G_{\delta}$ de $\mathbb{N}^{*}$. Assim, pelo Teorema 
1.26 (b), existe $G \subset \mathbb{T}$ aberto de $\mathbb{N}^{*}$ tal que $G \cap\left(\bigcup_{n \in \mathbb{Z}} K_{n, Y}\right)=\varnothing$.

Agora, seja $q \in G$. Suponha por absurdo que $p \in \beta \mathbb{Z}+q+p$. Como $\beta \mathbb{Z}+q+p=\overline{\mathbb{Z}+q+p}$, então $\bar{Y} \cap(\mathbb{Z}+q+p) \neq \varnothing$, logo tome $n \in \mathbb{Z}$ tal que $n+q+p \in \bar{Y}$. Ou seja, $q \in K_{n, Y}$. Contradição.

Corolário 2.56. Seja $p \in \mathbb{N}^{*} \backslash K(\beta \mathbb{N})$. Então existe uma sequência crescente $\left(x_{n}\right)_{n \geq 1}$ em $\mathbb{N}$ tal que: para todo $n \in \mathbb{N}, x_{n+1}$ é múltiplo de $x_{n}$ e de $n$ !; para todo $q \in\left\{x_{n}: n \in \mathbb{N}\right\}^{*}, p \notin \beta \mathbb{Z}+q+p$; $x_{1}=1$.

Demonstração. Tome $G$ como no Teorema anterior. Tome $E \subset \mathbb{N}$ infinito tal que $E^{*} \subset G$. Como $E^{*} \subset T$, segue que para todo $k \in \mathbb{N}, E \cap k \mathbb{N}$ é infinito. Assim, podemos recursivamente tomar $x_{1}=1$ e $x_{n+1} \in E \cap\left(x_{n} \cdot n !\right) \mathbb{N}$ tal que $x_{n+1}>x_{n}$. E como $\left\{x_{n}: n \geq 2\right\} \subset E$, então $\left\{x_{n}: n \in \mathbb{N}\right\}^{*} \subset G$, e portanto pelo Teorema anterior para todo $q \in\left\{x_{n}: n \in \mathbb{N}\right\}^{*}$ temos que $p \notin \beta \mathbb{Z}+q+p$.

Teorema 2.57. Seja p um elemento de $\mathbb{N}^{*} \backslash K(\beta \mathbb{N})$ e $X=\left\{x_{n}: n \in \mathbb{N}\right\}$ a imagem da sequência obtida no corolário acima. Então para todo $q \in X^{*}, q+p$ é cancelável à direita. E dados $q_{1}, q_{2} \in X^{*}$ distintos, então $\mathbb{N}^{*}+q_{1}+p \cap \mathbb{N}^{*}+q_{2}+p=\varnothing$.

Demonstração. Mostraremos que dados $q_{1}, q_{2}$, vale que $q_{1}+p \notin \beta \mathbb{N}+q_{2}+p$. Suponha por absurdo que, para algum $u \in \beta \mathbb{N}, q_{1}+p=u+q_{2}+p$. Como $q_{1} \in \bar{X}$, temos que $\overline{X+p} \cap \overline{\mathbb{N}+q_{2}+p} \neq \varnothing$. Pelo 1.26(c), temos que ou $x+p=v+q_{2}+p$ para algum $x \in X$ e algum $v \in \beta \mathbb{N}$, ou $t+p=n+q_{2}+p$ para algum $t \in \bar{X}$ e algum $n \in \mathbb{N}$. O primeiro caso implica que $p \in \beta \mathbb{Z}+q_{2}+p$, assim como o segundo se $t \in X$, um absurdo; logo $t \in X^{*}$. Aplicando o homomorfismo $\widetilde{\gamma_{m}}$ a ambos os lados e cancelando $\widetilde{\gamma_{m}}(p)$, temos que $\widetilde{\gamma_{m}}(t)=\gamma_{m}(n)+\widetilde{\gamma_{m}}\left(q_{2}\right)$, para todo $m \in \mathbb{N}$. Isso é um absurdo pois $\widetilde{\gamma_{m}}(t)=\widetilde{\gamma_{m}}\left(q_{2}\right)=0$ para todo $m \in \mathbb{N}$, já que $t, q_{2} \in X^{*} \subset \mathbb{T}$.

Logo, $q_{1}+p \notin \beta \mathbb{N}+q_{2}+p$.

No caso em que $q_{1}=q_{2}=$ : $q$, obtemos pelo Teorema 2.41 que $q$ é cancelável à direita.

No caso em que $q_{1} \neq q_{2}$, pelo Lema 2.54 , basta ver que $q_{1}+p \neq q_{2}+p$ para concluir que $(\beta \mathbb{N}+$ $\left.q_{1}+p\right) \cap\left(\beta \mathbb{N}+q_{2}+p\right)=\varnothing$ (e em particular a tese). Suponha então que $q_{1}+p=q_{2}+p$, e tome $X_{1}, X_{2} \subset X$ disjuntos e tais que $X_{1} \in q_{1}$ e $X_{2} \in q_{2}$. Temos que $\overline{X_{1}+p} \cap \overline{X_{2}+p} \neq \varnothing$, logo temos que $a+p=b+p$, para $a \in X_{1}$ e $b \in \overline{X_{2}}$, ou para $a \in \overline{X_{1}}$ e $b \in X_{2}$. Sem perda de generalidade, suponha $a \in X_{1}$ e $b \in \overline{X_{2}}$; caso $b \in X_{2}$, teríamos que $a=b \in X_{1} \cap X_{2}$, contradição. Logo $b \in X_{2}{ }^{*}$, do que $p=-a+b+p \in \beta \mathbb{Z}+b+p$, uma contradição pois $b \in X^{*}$.

Para o próximo teorema, precisaremos de duas funções $\varphi, \theta: \mathbb{N} \rightarrow \omega$ da seguinte maneira: $\varphi(n)=\max \left\{x_{m}: m \in \mathbb{N}\right.$ e $\left.x_{m} \mid n\right\}$, e $\theta(n)=n-\varphi(n)$. Como exigimos $x_{1}=1, \varphi$ está bem-definida e consequentemente também está $\theta$.

Agora, para todo $m \in \mathbb{N}$, para todo $z$ que é múltiplo de $x_{m+1}$, temos que $\varphi\left(x_{m}+z\right)=x_{m}$, pois $x_{m}\left|x_{m+1}\right| z$, mas $x_{m+1} \nmid x_{m}$; e $\operatorname{assim} \theta\left(x_{m}+z\right)=z$. Sejam agora $q \in X^{*}$ e $r \in \mathbb{T}$. Como $r$ é o 
limite de uma rede em $x_{m+1} \mathbb{N}$, segue que $\widetilde{\varphi}\left(x_{m}+r\right)=x_{m}$ e $\widetilde{\theta}\left(x_{m}+r\right)=r$, para todo $m \in \mathbb{N}$. E como $q$ é o limite de uma rede em $X$, segue que $\widetilde{\varphi}(q+r)=q$ e $\widetilde{\theta}(q+r)=r$.

Em geral, vale que, dados $s \in \mathbb{N}, x_{m}=\varphi(s)$, e $t$ múltiplo de $x_{m+1}$, temos que $\varphi(s+t)=x_{m}=\varphi(s)$ e $\operatorname{logo} \theta(s+t)=\theta(s)+t$. Usando redes novamente, temos que para todos $u \in \beta \mathbb{N}$ e todo $r \in \mathbb{T}$, $\widetilde{\varphi}(u+r)=\widetilde{\varphi}(u)$, е $\widetilde{\theta}(u+r)=\widetilde{\theta}(u)+r$.

Teorema 2.58. Seja $p$ um elemento de $\mathbb{N}^{*} \backslash K(\beta \mathbb{N})$ e $X=\left\{x_{n}: n \in \mathbb{N}\right\}$ a imagem da sequência obtida no corolário acima. Então para cada $q \in X^{*}$, o ideal à esquerda semiprincipal $\mathbb{N}^{*}+q+p$ é maximal com relação a ser um ideal à esquerda semiprincipal de $\mathbb{N}^{*}$ estritamente contido em $\mathbb{N}^{*}+p$. Portanto, $\mathbb{N}^{*}+p$ está imediatamente acima de $2^{\mathfrak{c}}$ ideais à esquerda semiprincipais de $\mathbb{N}^{*}$.

Demonstração. Começaremos provando a seguinte Afirmação: Se $r \in \mathbb{T}$ é tal que $r \notin \mathbb{N}^{*}+q+r$, então não existe $s \in \mathbb{N}^{*}$ tal que $\mathbb{N}^{*}+q+r \subsetneq \mathbb{N}^{*}+s \subsetneq \mathbb{N}^{*}+r$.

Suponha que tal $s$ exista. Pelo Lema 2.54 , como $\mathbb{N}^{*}+q+r \subsetneq \mathbb{N}^{*}+s$, então $q+r \in \beta \mathbb{N}+s$, $\operatorname{logo}$ tome $t \in \beta \mathbb{N}$ tal que $q+r=t+s$. Como $q \in X^{*}$, temos $\overline{X+r} \cap \overline{\mathbb{N}+s} \neq \varnothing$, logo existem $a \in X$ e $t^{\prime} \in \beta \mathbb{N}$ tais que $a+r=t^{\prime}+s$, ou existem $q^{\prime} \in X^{*}$ e $b \in \mathbb{N}$ tais que $q^{\prime}+r=b+s$. Na primeira possibilidade, temos $r=-a+t^{\prime}+s$; se $t^{\prime} \in \mathbb{N}, r \in \mathbb{Z}+s$; se $t^{\prime} \in \mathbb{N}^{*}, r \in \mathbb{N}^{*}+s$; em ambos os casos, $\mathbb{N}^{*}+r \subset \mathbb{N}^{*}+s$, uma contradição. Na segunda possibilidade, como $q^{\prime} \in X^{*} \subset \mathbb{T}$ e $r \in \mathbb{T}$, então $b+s=q^{\prime}+r \in \mathbb{T}$. Assim, como $q+r=t+s=(-b+t)+(b+s)$, aplicando $\widetilde{\theta}$, obtemos $r=\widetilde{\theta}(-b+t)+(b+s)$, e novamente segue que $\mathbb{N}^{*}+r \subset \mathbb{N}^{*}+s$, uma contradição.

Agora, provaremos, como desejamos, que vale a propriedade para $p$.

Primeiramente observe que, pelo teorema anterior, $q+p$ é cancelável à direita, e portanto $q+p \notin$ $\mathbb{N}^{*}+q+p$; dessa forma, $\mathbb{N}^{*}+q+p \subsetneq \mathbb{N}^{*}+p$. Assim, $p \notin \mathbb{N}^{*}+q+p$.

Construiremos uma sequência $\left(r_{n}\right)_{n \geq 1}$. Note que, para cada $m \in \mathbb{N},\left\{x \in \mathbb{Z}: \gamma_{m}(x)=\widetilde{\gamma_{m}}(-p)\right\} \in$ $-p$, logo, para cada $n \in \mathbb{N}, \bigcap_{m=1}^{n}\left\{x \in \mathbb{Z}: \gamma_{m}(x)=\widetilde{\gamma_{m}}(-p)\right\}$ é infinito. Assim, podemos tomar $r_{1} \in \mathbb{N}$ qualquer e então recursivamente $r_{n+1}$ tal que $\gamma_{m}\left(r_{n+1}\right)=\widetilde{\gamma_{m}}(-p)$ para todo $m \in\{1, \ldots, n+1\}$, e tal que $r_{n+1}>r_{n}+n$.

Tome $w \in\left\{r_{n}: n \in \mathbb{N}\right\}^{*}$. Como, dado $m \in \mathbb{N}, \gamma_{m}\left(r_{n}\right)=\widetilde{\gamma_{m}}(-p)$ para todo $n \geq m$, segue que $\widetilde{\gamma_{m}}(w)=\widetilde{\gamma_{m}}(-p)$ para todo $m \in \mathbb{N}$. Fixe $m \in \mathbb{N}$. Temos que $\widetilde{\gamma_{m}}(p+w)=\widetilde{\gamma_{m}}(p)+\widetilde{\gamma_{m}}(-p)$. Vale que $\left\{x \in \mathbb{Z}: \gamma_{m}(x)=\widetilde{\gamma_{m}}(p)\right\} \in p, \log \left\{y \in \mathbb{Z}: \gamma_{m}(y)=-\widetilde{\gamma_{m}}(p)\right\}=\left\{y \in \mathbb{Z}: \gamma_{m}(-y)=\widetilde{\gamma_{m}}(p)\right\}=$ $-\left\{x \in \mathbb{Z}: \gamma_{m}(x)=\widetilde{\gamma_{m}}(p)\right\} \in-p$, e portanto $\widetilde{\gamma_{m}}(-p)=-\widetilde{\gamma_{m}}(p)$. Logo $p+w \in \bigcap_{m \geq 1}{\widetilde{\gamma_{m}}}^{-1}[\{0\}]=\mathbb{T}$. Além disso, como $\left\{r_{n}: n \in \mathbb{N}\right.$ e $\left.r_{n+1}>r_{n}+k\right\} \supset\left\{r_{n}: n \geq k\right\}$ para todo $k \in \mathbb{N}$, pelo Teorema 2.42, $w$ é cancelável à direita. Isso implica: (1) que $p+w \notin \mathbb{N}^{*}+q+p+w$, pois $p \notin \mathbb{N}^{*}+q+p$; (2) dados $u, v \in \mathbb{N}^{*}, \mathbb{N}^{*}+u \subset \mathbb{N}^{*}+v \Leftrightarrow \mathbb{N}^{*}+u+w \subset \mathbb{N}^{*}+v+w$.

Como $p+w \in \mathbb{T}$ e $p+w \notin \mathbb{N}^{*}+q+p+w$, pela Afirmação, não existe $s \in \mathbb{N}^{*}$ tal que $\mathbb{N}^{*}+q+p+w \subsetneq \mathbb{N}^{*}+s \subsetneq \mathbb{N}^{*}+p+w$. Pela equivalência em (2), segue que não existe $s \in \mathbb{N}^{*}$ tal 
que $\mathbb{N}^{*}+q+p \subsetneq \mathbb{N}^{*}+s \subsetneq \mathbb{N}^{*}+p$.

Pelo Teorema acima, os ideais à esquerda da forma $\mathbb{N}^{*}+q+p$ para $q \in X^{*}$ são todos distintos, portanto acabamos de provar que $\mathbb{N}^{*}+p$ está imediatamente acima de $2^{\mathfrak{c}}$ ideais à esquerda semiprincipais de $\mathbb{N}^{*}$.

Assim, com $p$ e $q$ como no teorema acima, $q+p$ será cancelável à direita, e portanto pertence a $\mathbb{N}^{*} \backslash K(\beta \mathbb{N})$, de forma que se pode aplicar o teorema novamente. Recursivamente, obtemos uma sequência decrescente $\left(\mathbb{N}+p_{n}\right)_{n \geq 1}$ de ideais à esquerda semiprincipais. O próximo teorema nos mostra que é possível prolongar esse processo.

Teorema 2.59. Suponha que $\left(\mathbb{N}^{*}+p_{n}\right)_{n \geq 1}$ seja uma sequência estritamente decrescente de ideais à esquerda semiprincipais. Dado $q \in \overline{\left\{p_{n}: n \in \mathbb{N}\right\}} \backslash\left\{p_{n}: n \in \mathbb{N}\right\}$, então $\mathbb{N}^{*}+q$ é maximal com respeito a ser um ideal à esquerda semiprincipal contido em $\bigcap_{n \geq 1}\left(\mathbb{N}^{*}+p_{n}\right)$. Além disso, q é cancelável à direita.

Demonstração. Seja $q \in \overline{\left\{p_{n}: n \in \mathbb{N}\right\}} \backslash\left\{p_{n}: n \in \mathbb{N}\right\}$. Primeiramente vejamos que $q \in \mathbb{N}^{*}+p_{n}$ para todo $n \in \mathbb{N}$. Como $q \in \overline{\left\{p_{n}: n \in \mathbb{N}\right\}} \backslash\left\{p_{n}: n \in \mathbb{N}\right\}$, então $q$ é o limite de uma rede em $\left\{p_{n}: n \in \mathbb{N}\right\}$ que eventualmente está em $\left\{p_{r}: r>n\right\}$. Dado $r>n$, pelo Lema 2.54, $p_{r} \in \beta \mathbb{N}+p_{n}$. Porém, caso $p_{r} \in \mathbb{N}+p_{n}$, então $p_{n} \in \mathbb{Z}+p_{r}$ e $\operatorname{logo} \mathbb{N}^{*}+p_{n} \subset \mathbb{N}^{*}+p_{r}$, uma contradição. Logo $p_{r} \in \mathbb{N}^{*}+p_{n}$ e portanto $p_{r} \in \mathbb{N}^{*}+p_{n}$. Como $\mathbb{N}^{*}+p_{n}$ é fechado e $q \in \overline{\left\{p_{r}: r>n\right\}}$, então $q \in \mathbb{N}^{*}+p_{n}$. Assim, para todo $n \in \mathbb{N}, \mathbb{N}^{*}+q \subset \mathbb{N}^{*}+p_{n}$.

Provaremos agora a seguinte afirmação: $\operatorname{dados} q, q^{\prime} \in \overline{\left\{p_{n}: n \in \mathbb{N}\right\}} \backslash\left\{p_{n}: n \in \mathbb{N}\right\}$, vale que $q \notin$ $\beta \mathbb{N}+q^{\prime}$. Suponha por absurdo que haja tais $q, q^{\prime}$. Temos que $\overline{\left\{p_{n}: n \in \mathbb{N}\right\}} \cap \overline{\mathbb{N}+q^{\prime}} \neq \varnothing$, logo pelo Teorema 1.26(c), existe $n \in \mathbb{N}$ tal que $p_{n} \in \beta \mathbb{N}+q^{\prime}$, ou existem $q^{\prime \prime} \in \overline{\left\{p_{n}: n \in \mathbb{N}\right\}}$ e $m \in \mathbb{N}$ tal que $q^{\prime \prime}=m+q^{\prime}$. A primeira possibilidade implica que $\mathbb{N}^{*}+p_{n} \subset \mathbb{N}^{*}+\beta \mathbb{N}+q^{\prime} \subset \mathbb{N}^{*}+q^{\prime} \subset \mathbb{N}^{*}+p_{n+1}$, uma contradição. Na segunda, temos que $\overline{\left\{p_{n}: n \in \mathbb{N}\right\}} \cap \overline{\left\{m+p_{n}: n \in \mathbb{N}\right\}}$, logo existem $n \in \mathbb{N}$ e $q^{\prime \prime \prime} \in \overline{\left\{p_{n}: n \in \mathbb{N}\right\}}$ tais que $q^{\prime \prime \prime}=m+p_{n}$ ou $p_{n}=m+q^{\prime \prime \prime}$. Caso $q^{\prime \prime \prime} \in\left\{p_{n}: n \in \mathbb{N}\right\}$, tome $r \in \mathbb{N}$ tal que $q^{\prime \prime \prime}=p_{r}$. Temos: se $r=n$, então $p_{n}=m+p_{n}$, um absurdo (pois a função $x \mapsto x+m$ não possui pontos fixos pelo Teorema 1.26(a)); se $r \neq n$, temos que $p_{n} \in \mathbb{Z}+p_{r}$ e $p_{r} \in \mathbb{Z}+p_{n}$, do que segue que $\mathbb{N}^{*}+p_{n}=\mathbb{N}^{*}+p_{r}$, uma contradição. Logo $q^{\prime \prime \prime} \in \overline{\left\{p_{n}: n \in \mathbb{N}\right\}} \backslash\left\{p_{n}: n \in \mathbb{N}\right\}$ e $p_{n} \in \mathbb{Z}+q^{\prime \prime \prime}$, logo pelo visto acima, $\mathbb{N}^{*}+p_{n} \subset \mathbb{N}^{*}+q^{\prime \prime \prime} \subset \mathbb{N}^{*}+p_{n+1}$, uma contradição.

Portanto, vale a afirmação. No caso $q=q^{\prime}$, obtemos pelo Teorema 2.41 que $q$ é cancelável à direita. Agora, veremos que não existe $r \in \mathbb{N}^{*}$ tal que $\mathbb{N}^{*}+q \subsetneq \mathbb{N}^{*}+r \subset \bigcap_{n \geq 1}\left(\mathbb{N}^{*}+p_{n}\right)$. Suponha por absurdo que haja um tal $r$. Pelo Lema 2.54 e como $\mathbb{N}^{*}+q \subsetneq \mathbb{N}^{*}+r$, vale que $q \in \mathbb{N}^{*}+r$, logo seja $s \in \mathbb{N}^{*}$ tal que $q=s+r$. Temos que $\overline{\left\{p_{n}: n \in \mathbb{N}\right\}} \cap \overline{\mathbb{N}+r} \neq \varnothing$, logo pelo 1.26(c) existem $n \in \mathbb{N}$ e $s^{\prime} \in \beta \mathbb{N}$ tais que $p_{n}=s^{\prime}+r$, ou existem $q^{\prime} \in \overline{\left\{p_{n}: n \in \mathbb{N}\right\}}$ e $m \in \mathbb{N}$ tais que $q^{\prime}=m+r$. No primeiro caso, obtemos $\mathbb{N}^{*}+p_{n} \subset \mathbb{N}^{*}+r \subset \mathbb{N}^{*}+p_{n+1}$, uma contradição. No segundo, se $q^{\prime} \in\left\{p_{n}: n \in \mathbb{N}\right\}$, 
obtemos a mesma contradição do primeiro. Logo $q^{\prime} \in \overline{\left\{p_{n}: n \in \mathbb{N}\right\}} \backslash\left\{p_{n}: n \in \mathbb{N}\right\}$. Temos que $r=-m+q^{\prime}$, e portanto $q=s+r=-m+s+q^{\prime} \in \beta \mathbb{N}+q^{\prime}$. Isso contradiz a afirmação provada acima.

Observe acima que como $\left(\mathbb{N}^{*}+p_{n}\right)_{n \geq 1}$ é estritamente decrescente, então de fato $\mathbb{N}^{*}+q \neq \mathbb{N}^{*}+p_{n}$ para todo $n \in \mathbb{N}$. Assim, podemos a partir dos dois últimos teoremas fazer o passo limite enumerável na construção de tais cadeias. Mais precisamente, obtemos o seguinte corolário:

Corolário 2.60. Seja $p \in \mathbb{N}^{*} \backslash K(\beta \mathbb{N})$. Então $\mathbb{N}^{*}+p$ pertence a um segmento de uma cadeia maximal de ideais à esquerda semiprincipais de $\mathbb{N}^{*}$ que é reversamente bem-ordenado de tipo $\omega_{1}^{*}$ (isto é, de tipo $\omega_{1}$ para a ordem $\supset$ ).

Demonstração. Defina $p_{0}=p$. Suponha $\alpha<\omega_{1} \operatorname{com} p_{\beta}$ escolhidos para todos $\beta<\alpha<\omega_{1}$ de forma que $\left(\mathbb{N}^{*}+p_{\beta}\right)_{\beta<\alpha}$ seja uma cadeia estritamente decrescente, com cada $p_{\beta} \in \mathbb{N}^{*} \backslash K(\beta \mathbb{N})$. Caso $\alpha$ seja sucessor, aplique o Teorema 2.58 para $p_{\alpha-1}$ e obtenha $p_{\alpha} \in \mathbb{N}^{*}$ cancelável à direita e tal que $\mathbb{N}^{*}+p_{\alpha}$ é maximal com respeito a ser um ideal à esquerda semiprincipal estritamente contido em $\mathbb{N}^{*}+p_{\alpha-1}$.

Caso $\alpha$ seja limite, tome $\left(\gamma_{n}\right)_{n \geq 1}$ sequência crescente e cofinal em $\alpha$. Aplique o Teorema anterior para $\left(\mathbb{N}^{*}+p_{\gamma_{n}}\right)_{n \geq 1}$ e obtenha $p_{\alpha} \in \mathbb{N}^{*}$ cancelável à direita e tal que $\mathbb{N}^{*}+p_{\alpha}$ é maximal com respeito a ser um ideal à esquerda semiprincipal contido em $\bigcap_{n \geq 1}\left(\mathbb{N}^{*}+p_{\gamma_{n}}\right)$. Como $\left(\gamma_{n}\right)_{n \geq 1}$ é cofinal e $\left(\mathbb{N}^{*}+p_{\beta}\right)_{\beta<\alpha}$ é estritamente decrescente, temos que $\bigcap_{n \geq 1}\left(\mathbb{N}^{*}+p_{\gamma_{n}}\right)=\bigcap_{\beta<\alpha}\left(\mathbb{N}^{*}+p_{\beta}\right)$, e assim $\mathbb{N}^{*}+p_{\alpha}$ é maximal com respeito a ser um ideal à esquerda semiprincipal contido em $\bigcap_{\beta<\alpha}\left(\mathbb{N}^{*}+p_{\beta}\right)$. Assim, $\left(\mathbb{N}^{*}+p_{\alpha}\right)_{\alpha<\omega_{1}}$ é uma cadeia de ideais à esquerda semiprincipais de tipo de ordem $\omega_{1}{ }^{*}$. Um uso do Lema de Zorn nos permite tomar uma cadeia $\mathcal{C}$ maximal com relação a ser uma cadeia de ideais à esquerda semiprincipais que contém $\left\{\mathbb{N}^{*}+p_{\alpha}: \alpha<\omega_{1}\right\}$. Claramente, $\mathcal{C}$ também é maximal com relação a meramente ser uma cadeia de ideais à esquerda semiprincipais. Temos que $\left\{\mathbb{N}^{*}+p_{\alpha}: \alpha<\omega_{1}\right\}$ é um subconjunto de $\mathcal{C}$; suponha que não seja um segmento, isto é, suponha que existe $C \in \mathcal{C}$ tal que existem $\alpha<\beta<\omega_{1}$ tais que $\mathbb{N}^{*}+p_{\beta} \subsetneq C \subsetneq \mathbb{N}^{*}+p_{\alpha}$. Tome $\gamma<\omega_{1}$ o menor tal que $\mathbb{N}^{*}+p_{\gamma} \subsetneq C$ e note que $\gamma>0$; caso $\gamma$ seja sucessor, temos que $C \subsetneq \mathbb{N}^{*}+p_{\gamma-1}$, contrariando a maximalidade de $\mathbb{N}^{*}+p_{\gamma}$ como ideal à esquerda semiprincipal estritamente contido em $\mathbb{N}^{*}+p_{\gamma-1}$; caso $\gamma$ seja limite, temos que $C \subsetneq \mathbb{N}^{*}+p_{\delta}$ para todo $\delta<\gamma$, e portanto $C \subset \bigcap_{\delta<\gamma}\left(\mathbb{N}^{*}+p_{\delta}\right)$, contrariando a maximalidade de $\mathbb{N}^{*}+p_{\gamma}$ como ideal à esquerda semiprincipal contido em $\bigcap_{\delta<\gamma}\left(\mathbb{N}^{*}+p_{\delta}\right)$.

Agora, passaremos ao artigo "Chains of idempotents in $\beta N$ " para investigar resultados análogos para idempotentes não-minimais.

Definição 2.61. Seja $\left(x_{n}\right)_{n \geq 1}$ uma sequência crescente em $\mathbb{N}$ e seja $Y \subset \mathbb{N}$. 
(a) Para cada $m \in \mathbb{N}, R_{m, Y}:=\left\{\sum_{j=1}^{s} x_{n(j)}: s \in \mathbb{N}, x_{n(1)}>m,\{n(1), \ldots, n(s)\} \subset Y\right.$, e para cada $\left.i \in\{1, \ldots, s\}, x_{n(i)+1}>m+\sum_{j=1}^{i} x_{n(j)}\right\}$.

(b) $R_{Y}:=\bigcap_{m \geq 1} \overline{R_{m, Y}}$.

Lema 2.62. Sejam $Y \subset \mathbb{N} e\left(x_{n}\right)_{n \geq 1}$ uma sequência crescente e tal que $\left\{x_{n+1}-x_{n}: n \in Y\right\}$ é ilimitado. Então $R_{Y}$ é um subsemigrupo compacto de $\beta \mathbb{N}$. Além disso, dados $p, q \in \beta \mathbb{N}$, se $p+q \in R_{Y}$ e $q \in R_{Y}$, então $p \in R_{Y}$.

Demonstração. Como $\left\{x_{n+1}-x_{n}: n \in \mathbb{N}\right\}$ é ilimitado e $\left(x_{n}\right)_{n \geq 1}$ é crescente, então para cada $m \in \mathbb{N}$, $R_{m, Y} \neq \varnothing$ (basta tomar $n \in \mathbb{N}$ tal que $x_{n}>m$ e $\left.x_{n+1}-x_{n}>m+x_{n}\right)$. Como $\left(R_{m, Y}\right)_{m \geq 1}$ é uma sequência decrescente de subconjuntos de $\mathbb{N}$, segue que $R_{Y} \neq \varnothing$. Para ver que $R_{Y}$ é um semigrupo, sejam $p, q \in R_{Y}$ e seja $m \in \mathbb{N}$. Queremos ver que $p+q \in \overline{R_{m, Y}}$, ou seja, que $R_{m, Y} \in p+q$. Para isso, como $R_{m, Y} \in p$, basta ver que $R_{m, Y} \subset\left\{y \in \mathbb{N}:-y+R_{m, Y} \in q\right\}$. Assim, seja $y \in R_{m, Y}$ e tome $s \in \mathbb{N}$ e $n(1), \ldots, n(s)$ como na definição de $R_{m, Y}$ de forma que $y=\sum_{i=1}^{s} x_{n(i)}$. Considere $k=m+\sum_{i=1}^{s} x_{n(i)}$. Como $R_{k, Y} \in q$, então basta ver que $R_{k, Y} \subset-y+R_{m, Y}$. Seja $z \in R_{k, Y}$ e tome $t \in \mathbb{N}$ e $l(1), \ldots, l(t)$ como na definição de $R_{k, Y}$ de forma que $z=\sum_{i=1}^{t} x_{l(i)}$. Então, definindo $n(s+i)=l(i)$ para cada $i \in\{1, \ldots, t\}$, temos que $y+z=\sum_{i=1}^{s+t} x_{n(i)} \in R_{m, Y}$ (pois, para cada $\left.i \in\{1, \ldots, t\}, x_{n(s+i)+1}=x_{l(i)+1}>k+\sum_{j=1}^{i} x_{l(j)}=m+\sum_{j=1}^{s} x_{n(j)}+\sum_{j=s+1}^{s+i} x_{n(j)}\right)$.

Agora suponha que $p+q \in R_{Y}$ e que $q \in R_{Y}$. Para ver que $p \in R_{Y}$, seja $m \in \mathbb{N}$ e veremos que $R_{m, Y} \in p$. Considere $A=\left\{y \in \mathbb{N}:-y+R_{m, Y} \in q\right\}$. Como $R_{m, Y} \in p+q$, então $A \in p$, logo basta ver que $A \subset R_{m, Y}$. Seja $k \in A$; temos que $R_{k, Y} \in q$, logo $R_{k, Y} \cap\left(-k+R_{m, Y}\right) \neq \varnothing$. Assim, seja $z$ o elemento mínimo de $R_{k, Y} \cap\left(-k+R_{m, Y}\right)$. Como $z+k \in R_{m, Y}$, tome $s \in \mathbb{N}$ e $n(1), \ldots, n(s)$ como na definição de forma que $z+k=\sum_{i=1}^{s} x_{n(i)}$; e como $z \in R_{k, Y}$, tome $t \in \mathbb{N}$ e $l(1), \ldots, l(t)$ como na definição de forma que $z=\sum_{i=1}^{t} x_{l(i)}$. Afirmamos agora que $n(s)=l(t)$ : caso $n(s)>l(t)$, teríamos $z+k \geq x_{n(s)} \geq x_{l(t)+1}>k+\sum_{j=1}^{t} x_{l(j)}=k+z$, uma contradição; e caso $l(t)>n(s)$, teríamos $z \geq x_{l(t)} \geq x_{n(s)+1}>m+\sum_{j=1}^{s} x_{n(j)}=m+z+k$, outra contradição. Agora, vemos que $t=1$ : caso $t>1$, teríamos que $z-x_{l(t)}=\sum_{i=1}^{t-1} x_{l(i)} \in R_{k, Y}$ e que $z+k-x_{l(t)}=z+k-x_{n(s)}=$ $\sum_{i=1}^{s-1} x_{n(i)} \in R_{m, Y}$, de modo que $z-x_{l(t)} \in R_{k, Y} \cap\left(-k+R_{m, Y}\right)$, contrariando a minimidade de $z$. Assim, como $l(t)=n(s)$ e $t=1$, segue que $z=x_{l(1)}=x_{n(s)}$, e portanto $k=\sum_{i=1}^{s-1} x_{n(i)} \in R_{m, Y}$ como queríamos.

Teorema 2.63. Seja $p \in \mathbb{N}^{*}$ cancelável à direita. Então existe um subsemigrupo compacto $R$ de $\beta \mathbb{N}$ tal que $p \in R$ e $R \cap K(\beta \mathbb{N})=\varnothing$.

Demonstração. Pelo Teorema 2.42, tome $\left(x_{n}\right)_{n \geq 1}$ sequência crescente em $\mathbb{N}$ tal que, para cada $k \in \mathbb{N}, A_{k}:=\left\{x_{n}: n \in \mathbb{N}\right.$ e $\left.x_{n+1}>x_{n}+k\right\} \in p$. Note que $\left(A_{k}\right)_{k \geq 1}$ é uma sequência decrescente em $\mathcal{P}(\mathbb{N}), \log 0 \bigcap_{k \geq 1} A_{k}$ é um $G_{\delta}$ não-vazio de $\mathbb{N}^{*}$, de acordo com $1.26(\mathrm{~b})$. Assim, tome $q \in \bigcap_{k \geq 1} A_{k}$ 
distinto de $p$. Como $\left\{x_{n}: n \in \mathbb{N}\right\} \in p, q$ e a sequência é injetora, tome $Y, Z \subset \mathbb{N}$ disjuntos tais que $\left\{x_{n}: n \in Y\right\} \in p$ e $\left\{x_{n}: n \in Z\right\} \in q$.

Defina então $R=R_{Y}$. Primeiramente, vejamos que $p \in R_{Y}$. Observe que, para cada $k \in \mathbb{N}$, $\left\{x_{n}: n \in Y\right\} \cap A_{k} \cap(\mathbb{N} \backslash\{1, \ldots, k\}) \in p$, e $\left\{x_{n}: n \in Y\right\} \cap A_{k} \cap(\mathbb{N} \backslash\{1, \ldots, k\})=\left\{x_{n}: n \in Y, x_{n+1}>\right.$ $x_{n}+k$, e $\left.x_{n}>k\right\} \subset R_{k, Y}$, de forma que $R_{k, Y} \in p$. Agora, como $A_{k} \neq \varnothing$ para todo $k \in \mathbb{N}$, então $\left\{x_{n+1}-x_{n}: n \in \mathbb{N}\right\}$ é ilimitado, logo pelo Lema anterior $R_{Y}$ é um subsemigrupo compacto de $\beta \mathbb{N}$. Agora, suponha que $R \cap K(\beta \mathbb{N}) \neq \varnothing$ e tome $r \in R \cap K(\beta \mathbb{N})$. Como $K(\beta \mathbb{N})$ é a união de todos os ideais à esquerda minimais, tome $L$ ideal à esquerda minimal tal que $r \in L$. Segue que $q+r \in L, \operatorname{logo}$ $\beta \mathbb{N}+q+r$ é um ideal à esquerda contido em $L, \operatorname{logo} L=\beta \mathbb{N}+q+r$. Em particular, $r \in \beta \mathbb{N}+q+r$, logo tome $s \in \beta \mathbb{N}$ tal que $r=s+q+r$. Assim, $r \in R$ e $s+q+r \in R$, logo pelo Lema acima $s+q \in R$.

Considere $B=\left\{k+x_{n}: n \in Z, k \in \mathbb{N}\right.$, e $\left.x_{n+1}>x_{n}+k\right\}$. Afirmamos que $B \in s+q$; basta ver que $\{k \in \mathbb{N}:-k+B \in q\}=\mathbb{N}$. De fato, dado $k \in \mathbb{N}$, vale que $A_{k} \cap\left\{x_{n}: n \in Z\right\} \subset-k+B$ e $\operatorname{logo}-k+B \in q$. Temos então que $B \in s+q$, e $R_{1, Y} \in s+q$, logo tome $w \in B \cap R_{1, Y}$. Como $w \in B$, tome $k \in \mathbb{N}$ e $m \in Z$ tais que $w=k+x_{m}$ e $x_{m+1}>x_{m}+k$; como $w \in R_{1, Y}$, tome $t \in \mathbb{N}$ e $n(1), \ldots, n(t) \in Y$ tais que $w=\sum_{i=1}^{t} x_{n(i)}$ e, para cada $i \in\{1, \ldots, t\}, x_{n(i)+1}>x_{n(i)}+1$. Como $n(t) \in Y$ e $m \in Z$, então $n(t) \neq m$. Caso $n(t)>m$, temos $w \geq x_{n(t)} \geq x_{m+1}>x_{m}+k=w$, uma contradição; e caso $m>n(t)$, temos $w=k+x_{m}>x_{m} \geq x_{n(t)+1}>1+\sum_{j=1}^{t} x_{n(j)}=1+w$, outra contradição. Deste modo, $R \cap K(\beta \mathbb{N})=\varnothing$.

Teorema 2.64. Seja p um idempotente não-minimal de $\mathbb{N}^{*}$. Então existem $2^{\mathfrak{c}}$ idempotentes nãominimais imediatamente abaixo de $p$ com relação à ordem dos idempotentes $\leq$.

Demonstração. Tome pelo Corolário 2.56 a sequência $\left(x_{n}\right)_{n \geq 1}$, de imagem $X$. Pelo Teorema 2.57, dados $q_{1}, q_{2} \in X^{*}$ distintos, $\mathbb{N}^{*}+q_{1}+p \cap \mathbb{N}^{*}+q_{2}+p=\varnothing$. Portanto, como $\left|X^{*}\right|=2^{\mathfrak{c}}$, basta ver que para cada $q \in X^{*}$ existe $v \in \mathbb{N}^{*}+q+p$ tal que $v$ é maximal entre todos os idempotentes $s$ tais que $s<p$. Fixe então um $q \in X^{*}$.

O Teorema 2.57 também nos diz que $q+p$ é cancelável à direita. Pelo Teorema 2.63, tome $R$ subsemigrupo compacto e disjunto de $K(\beta \mathbb{N})$ e tal que $q+p \in R$. Vale que $R \cap\left(\mathbb{N}^{*}+q+p\right)$ é um subsemigrupo compacto, logo tome $t \in R \cap\left(\mathbb{N}^{*}+q+p\right)$ idempotente. Como $t \in \mathbb{N}^{*}+p, t=t+p$. Defina $r=p+t ; r+r=p+t+p+t=p+t+t=p+t \operatorname{logo} r$ é idempotente. Como $r=p+t+p$, então $r+p=p+r=r$, ou seja, $r \leq p$. Vale também que $r$ não é minimal. Se fosse, teríamos que $q+r \in K(\beta \mathbb{N})$, e $q+r=q+p+t$; como $q+p \in R$ e $t \in R$, segue que $q+r \in R \cap K(\beta \mathbb{N})$, uma contradição.

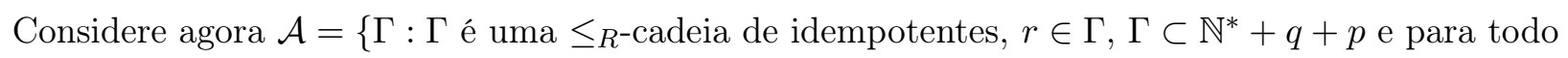
$s \in \Gamma, s \leq p\}$. Vale que $\{r\} \in \mathcal{A}$, e uma aplicação do Lema de Zorn nos permite tomar $\Gamma \in \mathcal{A}$ 
maximal.

Seja $L=\left\{u \in \mathbb{N}^{*}+q+p\right.$ : para todo $\left.s \in \Gamma, s=u+s\right\}$. Como $L=\left(\mathbb{N}^{*}+q+p\right) \cap \bigcap_{s \in \Gamma} \rho_{s}{ }^{-1}[\{s\}]$, então $L$ é compacto, e para ver que é não-vazio basta ver que $\left\{\mathbb{N}^{*}+q+p\right\} \cup\left\{\rho_{s}{ }^{-1}[\{s\}]: s \in \Gamma\right\}$ possui a p.i.f. Para isso, seja $F \in \mathcal{P}_{f}(\Gamma)$, e tome $u \in F$ o $\leq_{R}$-máximo de $F$. Vale então que $u \in\left(\mathbb{N}^{*}+q+p\right) \cap \bigcap_{s \in F} \rho_{s}^{-1}[\{s\}]$ (lembre que $s \leq_{R} u \Leftrightarrow s=u+s$, por definição). Observe também que cada $\rho_{s}{ }^{-1}[\{s\}]$ é um semigrupo. Portanto, $L$ é um subsemigrupo compacto, logo tome $u \in L$ idempotente e defina $v=p+u$. Como $u \in \mathbb{N}^{*}+p, u+p=u, \log v+v=p+u+p+u=$ $p+u+u=p+u$ e assim $v$ é um idempotente. Temos também que $v=p+u \in \mathbb{N}^{*}+q+p$ e dado $s \in \Gamma, v+s=p+u+s=p+s=s$ (pois $s \leq p$ ), $\operatorname{logo} v \in L$. Assim, $v$ não é minimal; caso $v \in K(\beta \mathbb{N})$, como $v \in L$ e $r \in \Gamma, r=v+r \in K(\beta \mathbb{N})$, uma contradição.

Como $v=p+u+p$, então $v \leq p$. Pelo Teorema $2.58, \mathbb{N}^{*}+q+p \subsetneq \mathbb{N}^{*}+p$, e portanto $p \notin \mathbb{N}^{*}+q+p \ni v$. Desse modo, $v<p$. Além disso, observe que $\Gamma \cup\{v\} \in \mathcal{A}$, logo pela maximalidade $v \in \Gamma$.

Por fim, mostraremos que $v$ é maximal entre todos os idempotentes $s$ tais que $s<p$. Primeiramente, mostraremos que $v$ é maximal entre todos os idempotentes em $\mathbb{N}^{*}+q+p$ tais que $s<p$. Suponha então $w \in \mathbb{N}^{*}+q+p$ idempotente tal que $v \leq w \leq p$. Para todo $s \in \Gamma$, vale que $s \leq_{R} v \leq_{R} w$, de forma que $\Gamma \cup\{w\} \in \mathcal{A}$; pela maximalidade, $w \in \Gamma$; assim, como $v \in L, w \leq_{R} v$; e já que $\Gamma$ é $\leq_{R^{-} \text {cadeia, } v}=w$.

Agora seja $w$ idempotente qualquer tal que $v \leq w \leq p$. Temos que $v \in\left(\mathbb{N}^{*}+q+p\right) \cap\left(\mathbb{N}^{*}+w\right)$, logo pelo Lema $2.54, \mathbb{N}^{*}+q+p \subset \mathbb{N}^{*}+w$ ou $\mathbb{N}^{*}+w \subset \mathbb{N}^{*}+q+p$. Caso $\mathbb{N}^{*}+w \subset \mathbb{N}^{*}+q+p$, então $w \in \mathbb{N}^{*}+q+p$ e pelo que foi feito acima, $v=w$. Caso $\mathbb{N}^{*}+q+p \subsetneq \mathbb{N}^{*}+w$, o Teorema 2.58 nos diz que $\mathbb{N}^{*}+w=\mathbb{N}^{*}+p$. Segue que $p=p+w$ pois $p \in \mathbb{N}^{*}+w$ e $w=p+w$ já que $w \leq p$, ou seja, $w=p$.

Note no teorema acima que $v \in \mathbb{N}^{*}+q+p \subsetneq \mathbb{N}^{*}+p$, e que portanto $p \notin \mathbb{N}^{*}+v$. Também será necessário para o corolário a seguir lembrar que dados $u, v$ idempotentes, $u \leq_{L} v$ se e somente se $u \in \mathbb{N}^{*}+v$; e definimos que $u<_{L} v$ se e só se $u \leq_{L} v$ e não vale $v \leq_{L} u$ (lembre que $\leq_{L}$ não é antissimétrica).

Corolário 2.65. Seja $p$ um idempotente não-minimal em $\mathbb{N}^{*}$. Então existe uma $\omega_{1}$-sequência $\left(p_{\sigma}\right)_{\sigma<\omega_{1}}$ de idempotentes tal que

(1) $p_{0}=p$;

(2) para cada $\sigma<\omega_{1}, p_{\sigma+1}$ é maximal entre todos os idempotentes menores do que $p_{\sigma}$;

(3) dados $\sigma<\tau<\omega_{1}, p_{\tau}<_{L} p_{\sigma}$.

Demonstração. Definimos $p_{0}=p$. Seja $0<\tau<\omega_{1}$ e suponha $\left(p_{\sigma}\right)_{\sigma<\tau}$ escolhidos de modo a satisfazer (1), (2) e (3) e tais que cada $p_{\sigma}$ é não-minimal. Caso $\tau$ seja sucessor, aplique o teorema 
anterior para $p_{\tau-1}$ e obtenha $p_{\tau}$ não-minimal e maximal entre todos os idempotentes menores do que $p_{\tau-1}$. Caso $\tau$ seja limite, aplique a técnica utilizada no Corolário 2.60 e obtenha $q \in \mathbb{N}^{*}$ cancelável à direita e tal que $\mathbb{N}^{*}+q \subset \bigcap_{\sigma<\tau}\left(\mathbb{N}^{*}+p_{\sigma}\right)$. Pelo Teorema 2.63, tome $R$ subsemigrupo compacto, disjunto de $K(\beta \mathbb{N})$ e tal que $q \in R$. Como $R \cap\left(\mathbb{N}^{*}+q\right)$ é subsemigrupo compacto, tome $p_{\tau}$ idempotente nele. Como $p_{\tau} \in R$, é não-minimal. E dado $\sigma<\tau$, temos que $p_{\tau} \in \mathbb{N}^{*}+p_{\sigma+1} \subsetneq \mathbb{N}^{*}+p_{\sigma}$, de forma que $p_{\tau}<_{L} p_{\sigma}$.

No teorema obtivemos uma $\omega_{1}$-sequência decrescente em $<_{L}$. Vale notar que não se pode ter sequências decrescentes em $<_{L}$ maiores do que $\mathfrak{c}$ : seja $\kappa$ cardinal e seja $\left(p_{\sigma}\right)_{\sigma<\kappa}$ uma tal sequência. Para cada $\sigma, \mathbb{N}^{*}+p_{\sigma}$ é um compacto que contém estritamente o compacto $\mathbb{N}^{*}+p_{\sigma+1}$; portanto, existe $U_{\sigma}$ um clopen de $\beta \mathbb{N}$ tal que $\mathbb{N}^{*}+p_{\sigma+1} \subset U_{\sigma}$ e tal que $\mathbb{N}^{*}+p_{\sigma} \not \subset U_{\sigma}$. Segue que os $U_{\sigma}$ são todos distintos; como os clopens de $\beta \mathbb{N}$ correspondem aos subconjuntos de $\mathbb{N}$, então $\kappa \leq \mathfrak{c}$. 


\section{Capítulo 3}

\section{A Âlgebra de $\beta S$}

Este capítulo tratará do contexto mais geral da álgebra na compactificação de Stone-Čech, quando consideramos um semigrupo $S$ qualquer e analisamos com mais minúcia a interação entre as estruturas algébrica e topológica. Propriedades como comutatividade e diversos níveis de cancelamento de $S$ se traduzem com frequência a propriedades topológicas de $\beta S$.

\subsection{Sobre a Raridade de Produtos em $S^{*}$}

Esta seção é o estudo do artigo de Neil Hindman e Dona Strauss "The Scarcity of Products in $\beta S \backslash S^{\prime \prime}[9]$ e suas referências que os sustentam.

Definição 3.1. Sejam $S$ um semigrupo e $A \subset S$.

(a) Á ú um conjunto solução à esquerda se existem $a, b \in S$ tais que $A=\{x \in S: a x=b\}$.

(b) A é um conjunto solução à direita se existem $a, b \in S$ tais que $A=\{x \in S: x a=b\}$.

Note que é imediato desta definição que: $S$ é cancelativo à esquerda se e somente se todo conjunto solução à esquerda é unitário; e $S$ é fracamente cancelativo à esquerda se e somente se todo conjunto solução à esquerda é finito. (Analogamente temos as afirmações à direita.)

Definição 3.2. Seja $S$ um semigrupo tal que $|S|=\kappa \geq \omega$.

(a) $S$ é fraquissimamente cancelativo à esquerda se dado $\mathcal{B}$ uma coleção de conjuntos solução à esquerda com $|\mathcal{B}|<\kappa$, vale que $|\bigcup \mathcal{B}|<\kappa$.

(b) $S$ é fraquissimamente cancelativo à direita se dado $\mathcal{B}$ uma coleção de conjuntos solução à direita $\operatorname{com}|\mathcal{B}|<\kappa$, vale que $|\bigcup \mathcal{B}|<\kappa$.

Note que se $\kappa$ for regular, então $S$ é fraquissimamente cancelativo à esquerda se e somente se cada conjunto solução à esquerda tiver cardinalidade menor do que $\kappa$. Assim, se $S$ for enumerável a noção coincide com a de fracamente cancelativo à esquerda. 
A maior parte do artigo (Seção 2) trata de encontrar condições suficientes para que $S^{*} S^{*}$ seja nunca-denso em $S^{*}$; serão mostradas também que algumas dessas condições não são necessárias, e contraexemplos que mostram que certas condições mais fracas para $S$ enumerável falham para cardinalidades maiores.

Para encontrar tais condições, há de valer em primeiro lugar que $S^{*} S^{*} \subset S^{*}$. Lembre que pelo Teorema 1.40(a), se $S$ for fracamente cancelativo à esquerda então $S^{*}$ é um ideal à esquerda de $\beta S$ e se $S$ for cancelativo à direita então $S^{*}$ é um ideal à direita. Ambas as condições implicam que $S^{*}$ é um subsemigrupo e são satisfeitas, por exemplo, se $S$ puder ser (algebricamente) imerso em um grupo.

Em primeiro lugar, temos o seguinte teorema, que é o 6.35 do livro [1].

Teorema 3.3. Seja $S$ um semigrupo enumerável que é cancelativo à direita e fracamente cancelativo à esquerda. Então $S^{*} S^{*}$ é nunca-denso em $S^{*}$.

Demonstração. Notemos que: $\operatorname{se} \operatorname{int}\left(\operatorname{cl}\left(S^{*} S^{*}\right)\right) \neq \varnothing$, então existe $T \subset S$ infinito tal que $T^{*} \subset$ $\operatorname{cl}\left(S^{*} S^{*}\right)$, e portanto para todo $V \subset T$ infinito, $V^{*} \subset \operatorname{cl}\left(S^{*} S^{*}\right)$, e assim $V^{*} \cap S^{*} S^{*} \neq \varnothing$.

Dessa forma, por contraposição, provaremos que para todo $T \subset S$ infinito existe $V \subset T$ infinito tal que $V^{*} \cap S^{*} S^{*}=\varnothing$.

Enumere $S$ como $\left\{s_{n}: n \in \mathbb{N}\right\}$. Seja $T \subset S$ infinito. Produziremos uma sequência injetora $\left(t_{n}\right)_{n \geq 1}$ em $T$ tal que as equações $s_{m} x=t_{n}$ e $s_{m^{\prime}} x=t_{n^{\prime}}$ não possuem solução simultânea se $m<n, m^{\prime}<n^{\prime}$ e $n<n^{\prime}$. No primeiro passo, tomamos $t_{1} \in T$ qualquer. Suponha $t_{1}, \ldots, t_{k}$ escolhidos. Considere $X=\left\{x \in S: s_{m} x=t_{n}\right.$ para algum $\left.m, n \leq k\right\}$. Como $S$ é fracamente cancelativo à esquerda, $X$ é finito, logo tome $t_{k+1} \in T \backslash\left(\bigcup_{m=1}^{k} s_{m} X \cup\left\{t_{1}, \ldots, t_{k}\right\}\right)$. Desse modo, obtemos a sequência injetora desejada.

Defina $V=\left\{t_{n}: n \in \mathbb{N}\right\}$. Vale que dados $p, q \in S^{*}, p \cdot q \notin V^{*}$. Suponha, por absurdo, que $p \cdot q \in V^{*}$. Considere $P=\left\{s \in S: s^{-1} V \in q\right\} . P \in p$. Tome $m<m^{\prime}$ tais que $s_{m}, s_{m^{\prime}} \in P$. Considere $Q=\left\{x \in S: s_{m} x, s_{m^{\prime}} x \in\left\{t_{i}: i>m^{\prime}\right\}\right\}$. Vale que $Q \in q$, pois $s_{m}, s_{m^{\prime}} \in P$ e $Q=$ $\left(s_{m}^{-1}\left(V \backslash\left\{t_{1}, \ldots, t_{m^{\prime}}\right\}\right)\right) \cap\left(s_{m^{\prime}}^{-1}\left(V \backslash\left\{t_{1}, \ldots, t_{m^{\prime}}\right\}\right)\right)$, usando novamente que $S$ é fracamente cancelativo à esquerda. Assim, tome $x \in Q$. Existem $n, n^{\prime}>m^{\prime}$ tais que $s_{m} x=t_{n}$ e $s_{m^{\prime}} x=t_{n^{\prime}}$. Caso $n \neq n^{\prime}$, haveria uma violação da propriedade com que a sequência $\left(t_{n}\right)_{n \geq 1}$ foi construída; $\operatorname{logo} n=n^{\prime}$. Ou seja, $s_{m} x=s_{m^{\prime}} x$. Como $S$ é cancelativo à direita, $s_{m}=s_{m^{\prime}}$; absurdo.

Vale notar que as condições de teorema não podem ser grandemente enfraquecidas: por exemplo, o semigrupo $(\mathbb{N}, \vee)$, em que $n \vee m=\max \{n, m\}$, é fracamente cancelativo à esquerda e à direita, e é fácil verificar (via redes) que dados $p \in \beta \mathbb{N}$ e $q \in \mathbb{N}^{*}, p \vee q=q$, de forma que $\mathbb{N}^{*} \vee \mathbb{N}^{*}=\mathbb{N}^{*}$.

Agora veremos que condição suficiente foi encontrada para semigrupos de quaisquer cardinalidades. 
Teorema 3.4. Seja $S$ um semigrupo infinito e algebricamente imersivel em um grupo G. Então $S^{*} \cap\left(G^{*} G^{*}\right)$ é nunca-denso em $S^{*}$. Em particular $S^{*} S^{*}$ é nunca-denso em $S^{*}$.

Demonstração. Como no teorema anterior, provaremos que dado $T \subset S$ infinito existe $V \subset T$ infinito tal que $V^{*} \cap G^{*} G^{*}=\varnothing$. Seja $T \subset S$ infinito então. Tome $t_{1} \in T$. Definidos $t_{1}, \ldots, t_{s-1}$, tome $t_{s} \in T \backslash\left\{t_{r} t_{n}{ }^{-1} t_{m}: m, n, r<s\right\}$. Note que $t_{s} \notin\left\{t_{m}: m \in\{1, \ldots, s-1\}\right\}$, de forma que a sequência obtida é injetora. Afirmamos que $V=\left\{t_{s}: s \in \mathbb{N}\right\}$ é o conjunto desejado. Suponha que $p, q \in G^{*}$ e $V \in p \cdot q$. Vale que $\left\{x \in G: x^{-1} V \in q\right\} \in p$, logo tome $x_{1}, x_{2}$ distintos tais que $x_{1}{ }^{-1} V, x_{2}{ }^{-1} V \in q$. Tome $y \in x_{1}{ }^{-1} V \cap x_{2}{ }^{-1} V$, e tome $n, r \in \mathbb{N}$ tais que $x_{1} y=t_{n}$ e $x_{2} y=t_{r}$. Agora, como $\left\{w \in S: x_{1} w \in\left\{t_{1}, \ldots, t_{\max \{n, r\}}\right\}\right\}$ e $\left\{w \in S: x_{2} w \in\left\{t_{1}, \ldots, t_{\max \{n, r\}}\right\}\right\}$ são finitos e $x_{1}{ }^{-1} V \cap x_{2}{ }^{-1} V$ é infinito, tome $w \in x_{1}{ }^{-1} V \cap x_{2}{ }^{-1} V$ tal que $x_{1} w=t_{m}$ e $x_{2} w=t_{s}$, com $m, s>\max \{n, r\}$. Sem perda de generalidade, $s>m$. Temos então: $t_{s} t_{m}{ }^{-1}=x_{2} x_{1}{ }^{-1}=t_{r} t_{n}{ }^{-1}$, de forma que $t_{s}=t_{r} t_{n}{ }^{-1} t_{m}$, contradizendo a construção da sequência. Logo $V^{*} \cap G^{*} G^{*}=\varnothing$.

Nesse teorema, como $S$ estava imerso em $G, S$ era cancelativo. O próximo teorema nos dá um exemplo de semigrupo não-cancelativo tal que $S^{*} S^{*}$ é nunca-denso em $S^{*}$.

Teorema 3.5. Considere $S=\{f: D \rightarrow \mathbb{N} \mid D \subset \mathbb{N}, D$ é cofinito e $f$ é uma bijeção $\}$, munido da operação de composição. Então $S$ é um semigrupo, $|S|=\mathfrak{c}, S$ não é cancelativo, é cancelativo à direita, fracamente cancelativo à esquerda e $S^{*} S^{*}$ é nunca-denso em $S^{*}$.

Demonstração. Começaremos vendo que $S$ é um semigrupo. Dadas $f, g \in S$, como não temos em geral que im $f \subset$ dom $g$, devemos tomar cuidado ao considerarmos $g \circ f$ - por exemplo, não podemos dizer diretamente que $g \circ f$ será uma bijeção entre $\operatorname{dom} f$ e $\mathbb{N}$. Mas veremos que esta relação de fato é uma bijeção entre um cofinito e $\mathbb{N}$. Denote $\operatorname{dom} f=D$ e $\operatorname{dom} g=E$.

Primeiramente, note que usando a definição de composição de relações, temos que $g \circ f=\{(d, x)$ : $f(d) \in E$ e $g(f(d))=x\}$. E é cofinito, e $f$ é bijetora, logo $f^{-1}[E]$ é cofinito. Defina $D^{\prime}=f^{-1}[E]$. Vale que $D^{\prime}=\operatorname{dom} g \circ f$, pois: dado $d \in D^{\prime}, f(d) \in E$, logo $(d, g(f(d))) \in g \circ f$; e se $d \in \operatorname{dom} g \circ f$, $f(d) \in E$, ou seja, $d \in D^{\prime}$. Vejamos que $g \circ f$ é função. Suponha $\left(d, x_{1}\right),\left(d, x_{2}\right) \in g \circ f$. Temos que $x_{1}=g(f(d))=x_{2}$; usando que $f$ é função e que $g$ é função, temos que $x_{1}=x_{2}$. Agora podemos usar que $g \circ f: D^{\prime} \rightarrow \mathbb{N}$ é função. Para ver que é sobrejetora, tome $x \in \mathbb{N}$; como $g$ é sobrejetora, tome $e \in E$ tal que $g(e)=x$; e como $f$ é sobrejetora, tome $d \in D$ tal que $f(d)=e$; vale então que $d \in D^{\prime}$ e $g(f(d))=x$. Para ver que é injetora, suponha $g\left(f\left(d_{1}\right)\right)=g\left(f\left(d_{2}\right)\right)$; como $g$ é injetora, vale que $f\left(d_{1}\right)=f\left(d_{2}\right)$; como $f$ é injetora, $d_{1}=d_{2}$. Assim, $g \circ f: D^{\prime} \rightarrow \mathbb{N}$ é uma bijeção com $D^{\prime} \subset \mathbb{N}$ cofinito, definida por $g \circ f(x)=g(f(x))$ para todo $x \in D^{\prime}$. (Como a composição de relações é associativa, temos por fim que $S$ é um semigrupo.)

Agora, $|S|=\mathfrak{c}:$ Note que $S=\bigcup_{\substack{D \subset \mathbb{N} \\ D \text { cofinito }}}\{f: D \rightarrow \mathbb{N}: f$ é bijeção $\}$. Como $\{D \subset \mathbb{N}: D$ é cofinito $\}$ é 
enumerável e para cada $D, \mid\{f: D \rightarrow \mathbb{N}: f$ é bijeção $\} \mid=\mathfrak{c}$, então $|S|=\omega \cdot \mathfrak{c}=\mathfrak{c}$.

Para ver que $S$ é fracamente cancelativo à esquerda: dados $f, g \in S$, trata-se de ver que $\{h \in$ $S: g \circ f=g \circ h\}$ é finito. Seja tal $h$. Tome $E, D_{f}, D_{h}$ tais que $g: E \rightarrow \mathbb{N}, f: D_{f} \rightarrow \mathbb{N}$ e $h: d_{h} \rightarrow \mathbb{N}$. Como $g \circ f=g \circ h$, vale que $f^{-1}[E]=h^{-1}[E]=D^{\prime}$, e que para todo $x \in D^{\prime}$, $g(f(x))=g(h(x))$. Ou seja, $f$ e $h$ coincidem sobre $D^{\prime}$ que é cofinito. Ademais, como $h$ é bijeção, vale que $h\left[D_{h} \backslash D^{\prime}\right]=h\left[D_{h}\right] \backslash h\left[D^{\prime}\right]=\mathbb{N} \backslash E$ que é finito. Assim, podemos identificar cada $h$ tal que $g \circ f=g \circ h$ com uma bijeção entre $D_{h} \backslash D^{\prime}$ e $\mathbb{N} \backslash E$. Como $D_{h} \supset f^{-1}[E]$ e este é cofinito, temos um número finito de possibilidades para $D_{h}$. Mais precisamente, podemos injetar $\{h \in S: g \circ f=g \circ h\}$ em $\bigcup\left\{b: D \backslash f^{-1}[E] \rightarrow \mathbb{N} \backslash E: b\right.$ é bijeção $\}$ - o conjunto índice é finito e cada conjunto da $f^{-1}[E] \subset D \subset \mathbb{N}$ união é finito.

Agora, que $S$ é cancelativo à direita: suponha $g_{1} \circ f=g_{2} \circ f$, com $f: D \rightarrow \mathbb{N}, g_{1}: E_{1} \rightarrow \mathbb{N}$ e $g_{2}: E_{2} \rightarrow \mathbb{N}$. Como $g_{1} \circ f=g_{2} \circ f$, seus domínios são iguais, ou seja, $f^{-1}\left[E_{1}\right]=f^{-1}\left[E_{2}\right]$. Seja $e \in E_{1}$. Tome $d \in D$ tal que $e=f(d) ; d \in f^{-1}\left[E_{1}\right]=f^{-1}\left[E_{2}\right], \operatorname{logo} e=f(d) \in E_{2}$. Temos então: $g_{1}(e)=g_{1}(f(d))=g_{2}(f(d))=g_{2}(e)$. Ou seja, dado $e \in E_{1}, e \in E_{2}$ e $g_{1}(e)=g_{2}(e)$. Analogamente, dado $e \in E_{2}$, $e \in E_{1}$ e $g_{2}(e)=g_{1}(e)$. Portanto, $g_{1}=g_{2}$.

Um exemplo para ver que $S$ não é cancelativo à esquerda: $g: \mathbb{N} \backslash\{1,2\} \rightarrow \mathbb{N}$ dado por $g(n)=n-2$; $f: \mathbb{N} \backslash\{1\} \rightarrow \mathbb{N}$ dado $f(n)=n-1 ; h: \mathbb{N} \backslash\{3\} \rightarrow \mathbb{N}$ dado por $h(n)=n-1$ se $n \geq 4, h(1)=1$ e $h(2)=2$; temos que $g \circ f=g \circ h$.

Antes de provar que $S^{*} S^{*}$ é nunca-denso precisaremos de duas propriedades:

(*) Sejam $t_{n}, t_{r}, t_{m} \in S$. Então $\left|\mathbb{N} \backslash\left\{a \in \operatorname{dom} t_{m}: t_{n}{ }^{-1}\left(t_{m}(a)\right) \in \operatorname{dom} t_{r}\right\}\right| \leq\left|\mathbb{N} \backslash \operatorname{dom} t_{m}\right|+\mid \mathbb{N} \backslash \operatorname{dom}$ $t_{r} \mid$. Em particular, $\left\{a \in \operatorname{dom} t_{m}: t_{n}{ }^{-1}\left(t_{m}(a)\right) \in \operatorname{dom} t_{r}\right\}$ é cofinito.

(**) Sejam $A \subset \mathbb{N}$ cofinito e $v: A \rightarrow \mathbb{N}$ injetora. Então $C=\left\{t \in S: A \subset \operatorname{dom} t\right.$ e $\left.\left.t\right|_{A}=v\right\}$ é finito. Prova de $(*): \mathbb{N} \backslash\left\{a \in \operatorname{dom} t_{m}: t_{n}{ }^{-1}\left(t_{m}(a)\right) \in \operatorname{dom} t_{r}\right\}=\mathbb{N} \backslash \operatorname{dom} t_{m} \cup\left\{a \in \operatorname{dom} t_{m}: t_{n}{ }^{-1}\left(t_{m}(a)\right) \in\right.$ $\mathbb{N} \backslash$ dom $\left.t_{r}\right\}$. Como $t_{n}{ }^{-1} \circ t_{m}$ é uma função injetora e $\mathbb{N} \backslash$ dom $t_{r}$ é finito, segue que $\mid\{a \in$ dom $\left.t_{m}: t_{n}{ }^{-1}\left(t_{m}(a)\right) \in \mathbb{N} \backslash \operatorname{dom} t_{r}\right\}|=|\left(t_{n}{ }^{-1} \circ t_{m}\right)^{-1}\left[\mathbb{N} \backslash \operatorname{dom} t_{r}\right]|\leq| \mathbb{N} \backslash \operatorname{dom} t_{r} \mid$. Disso segue $(*)$.

Prova de (**): note que cada $t \in C$ determina uma bijeção entre (dom $t) \backslash A$ e $\mathbb{N} \backslash v[A]$, ou seja, podemos injetar $C$ em $\bigcup_{A \subset D \subset \mathbb{N}}\{b: D \backslash A \rightarrow \mathbb{N} \backslash v[A]: b$ é bijeção\}, e este conjunto é finito.

Agora, vejamos que $S^{*} S^{*}$ é nunca-denso em $S^{*}$. Suponha por absurdo $V \subset S$ infinito tal que $V^{*} \subset \operatorname{cl} S^{*} S^{*}$. Produziremos uma sequência injetora $\left(t_{n}\right)_{n \geq 1}$ em $V$ tal que dados $s>1$ e $n, r, m<s$, então não existem $x_{1}, x_{2}, y, w \in S$ tais que $x_{1} y=t_{n}, x_{2} y=t_{r}, x_{1} w=t_{m}$ e $x_{2} w=t_{s}$.

Tome $t_{1} \in V$. Suponha escolhidos $t_{1}, \ldots, t_{s-1}$. Para cada $n, r, m<s$, defina $D_{n, r, m}=\{t \in S$ : existem $x_{1}, x_{2}, y, w \in S$ tais que $x_{1} y=t_{n}, x_{2} y=t_{r}, x_{1} w=t_{m}$ e $\left.x_{2} w=t\right\}$. Logo, para definir $t_{s}$, basta mostrar que $V \backslash\left(\left\{t_{n}: n<s\right\} \cup \bigcup_{n, r, m<s} D_{n, r, m}\right) \neq \varnothing$. De fato, mostraremos que cada $D_{n, r, m}$ 
é finito. Para ver isso, mostraremos que dados $t \in D_{n, r, m}$, se $a \in \operatorname{dom} t_{m}$ e $t_{n}{ }^{-1}\left(t_{m}(a)\right) \in \operatorname{dom} t_{r}$, então $t(a)=t_{r}\left(t_{n}{ }^{-1}\left(t_{m}(a)\right)\right)$ - pois assim, definindo $A=\left\{a \in \operatorname{dom} t_{m}: t_{n}{ }^{-1}\left(t_{m}(a)\right) \in \operatorname{dom} t_{r}\right\}$ e $v=\left.t_{r} \circ t_{n}{ }^{-1} \circ t_{m}\right|_{A}$, podemos usar $(*)$ e $(* *)$ e concluir que $D_{n, m, r} \subset C$.

Assim, seja $t \in D_{n, r, m}$ e tome $x_{1}, x_{2}, y, w \in S$ tais que $x_{1} y=t_{n}, x_{2} y=t_{r}, x_{1} w=t_{m}$ e $x_{2} w=t$; seja $a \in \operatorname{dom} t_{m}$ tal que $t_{n}{ }^{-1}\left(t_{m}(a)\right) \in \operatorname{dom} t_{r}$. Como $a \in \operatorname{dom} t_{m}, \operatorname{temos} t_{m}(a)=$ $x_{1}(w(a))$, logo $w(a)=x_{1}{ }^{-1}\left(t_{m}(a)\right)$. Como $t_{n}{ }^{-1}\left(t_{m}(a)\right) \in \operatorname{dom} t_{n}$, temos $t_{n}\left(t_{n}{ }^{-1}\left(t_{m}(a)\right)\right)=t_{m}(a)$ e $t_{n}\left(t_{n}{ }^{-1}\left(t_{m}(a)\right)\right)=x_{1}\left(y\left(t_{n}{ }^{-1}\left(t_{m}(a)\right)\right)\right)$, do que segue $y\left(t_{n}{ }^{-1}\left(t_{m}(a)\right)\right)=x_{1}{ }^{-1}\left(t_{m}(a)\right)=w(a)$. Por fim, como $t_{n}^{-1}\left(t_{m}(a)\right) \in \operatorname{dom} t_{r}$, temos $t_{r}\left(t_{n}{ }^{-1}\left(t_{m}(a)\right)\right)=x_{2}\left(y\left(t_{n}{ }^{-1}\left(t_{m}(a)\right)\right)\right)=x_{2}(w(a))=t(a)$, como pretendíamos.

Assim, considere $A=\left\{t_{n}: n \in \mathbb{N}\right\} . A^{*} \cap S^{*} S^{*} \neq \varnothing$, logo tome $p, q \in S^{*}$ tal que $A \in p \cdot q$. $\left\{x \in S: x^{-1} A \in q\right\} \in p$, logo tome $x_{1}, x_{2}$ distintos tais que $x_{1}{ }^{-1} A \cap x_{2}{ }^{-1} A \in q$, e portanto é infinito. Tome $y \in x_{1}^{-1} A \cap x_{2}{ }^{-1} A$, e tome $n, r$ tais que $x_{1} y=t_{n}$ e $x_{2} y=t_{r}$. S é cancelativo à direita, $\operatorname{logo} n \neq r$; assuma s.p.g. que $n<r$. Como $S$ é fracamente cancelativo à esquerda, o conjunto $\left\{z \in S: x_{1} z=t_{p}\right.$ ou $x_{2} z=t_{p}$ para algum $\left.p \leq r\right\}$ é finito, logo podemos tomar $w \in x_{1}{ }^{-1} A \cap x_{2}{ }^{-1} A$ tal que $x_{1} w=t_{m}$ e $x_{2} w=t_{s} \operatorname{com} m, s>r$. Agora, caso $m<s$, temos $n<r<m<s$ e assim $t_{s} \in D_{n, r, m}$, contradição. E caso $s<m$, temos $n<r<s<m$ e assim $t_{m} \in D_{n, r, s}$, contradição.

Lema 3.6. Seja $S$ um semigrupo infinito e suponha que $S^{*}$ é um subsemigrupo de $\beta S$. São equivalentes:

(a) $S^{*} S^{*}$ não é nunca-denso em $S^{*}$.

(b) Existe $V \in[S]^{\omega}$ tal que para todo $A \in[V]^{\omega}$ existem $\left(x_{n}\right)_{n \geq 1}$ e $\left(y_{n}\right)_{n \geq 1}$ sequências injetoras em $S$ tais que, sempre que $n>k, x_{k} y_{n} \in A$.

Demonstração. (a) $\Rightarrow(\mathrm{b})$ : Tome $V \subset S$ enumerável tal que $V^{*} \subset \operatorname{cl} S^{*} S^{*}$. Dado $A \subset V$ infinito, $A^{*} \cap S^{*} S^{*} \neq \varnothing$, logo tome $p, q \in S^{*}$ tal que $A \in p \cdot q .\left\{x \in S: x^{-1} A \in q\right\} \in p$ e portanto é infinito. Tome $\left(x_{n}\right)_{n \geq 1}$ sequência injetora em $\left\{x \in S: x^{-1} A \in q\right\}$. Tome então $y_{1} \in S$ qualquer e definidos $y_{1}, \ldots, y_{n}$ tome $y_{n+1} \in\left(\bigcap_{k=1}^{n} x_{k}{ }^{-1} A\right) \backslash\left\{y_{1}, \ldots, y_{n}\right\}$.

(b) $\Rightarrow\left(\right.$ a): Tome $V$ dado pela hipótese. Afirmamos que $V^{*} \subset \operatorname{cl} S^{*} S^{*}$. Seja $r \in V^{*}$; tome $A \in r$ tal que $A \subset V$. Quero ver que $A^{*} \cap S^{*} S^{*} \neq \varnothing$. Tome $\left(x_{n}\right)_{n \geq 1}$ e $\left(y_{n}\right)_{n \geq 1}$ dadas pela hipótese. Tome $p \in S^{*}$ tal que $\left\{x_{n}: n \in \mathbb{N}\right\}$ e $q \in S^{*}$ tal que $\left\{\left\{y_{n}: n>k\right\}: k \in \mathbb{N}\right\} \subset q$. Afirmação: $A \in p \cdot q$. Para ver isso, basta ver que $\left\{x_{k}: k \in \mathbb{N}\right\} \subset\left\{x \in S: x^{-1} A \in q\right\}$. Seja $k \in \mathbb{N}$ então. Note então que, pela propriedade das sequências, temos que $\left\{y_{n}: n>k\right\} \subset x_{k}{ }^{-1} A$, e portanto $x_{k}{ }^{-1} A \in q$.

O Teorema 3.5 nos mostrou que a hipótese de cancelamento não é necessária para que $S^{*} S^{*}$ seja nunca-denso em $S^{*}$. Agora veremos um exemplo que mostra que o cancelamento também não é suficiente caso $S$ não seja enumerável. 
Teorema 3.7. Existe um semigrupo cancelativo $S$ tal que $|S|=\mathfrak{c}$ e $S^{*} S^{*}$ não é nunca-denso em $S^{*}$.

Demonstração. Seja $L=\left\{z_{n}: n \in \mathbb{N}\right\} \cup\left\{x_{\sigma, k}: \sigma<\mathfrak{c}\right.$ e $\left.k \in \mathbb{N}\right\} \cup\left\{y_{\sigma, k}: \sigma<\mathfrak{c}\right.$ e $\left.k \in \mathbb{N}\right\}$, com todos os $z_{n}, x_{\sigma, k}$ e $y_{\sigma, k}$ distintos entre si. Defina $V=\left\{z_{n}: n \in \mathbb{N}\right\}$. Enumere $[V]^{\omega}=\left\{A_{\sigma}: \sigma<\mathfrak{c}\right\}$ e, para cada $\sigma<\mathfrak{c}$, enumere $A_{\sigma}=\left\{w_{\sigma, k, n}: k \geq 1\right.$ e $\left.n \geq k+1\right\}$.

Considere $S$ o conjunto de todas as palavras $a_{1} \ldots a_{t}$, com cada $a_{j} \in L$, tais que não existem $i<t$, $\sigma<\mathfrak{c}$, e $k<n$ tais que $a_{i}=x_{\sigma, k}$ e $a_{i+1}=y_{\sigma, n}$. Dadas $u=a_{1} \ldots a_{t}$ e $v=b_{1} \ldots b_{s}$ em $S$, defina $u \cdot v$ pela concatenação de $u$ e $v$, a não ser que existam $\sigma<\mathfrak{c}$ e $k<n$ tais que $a_{t}=x_{\sigma, k}$ e $b_{1}=y_{\sigma, n}$; nesse caso, $u \cdot v=a_{1} \ldots a_{t-1} w_{\sigma, k, n} b_{2} \ldots b_{s}$ (sendo que $a_{1} \ldots a_{t-1}$ é a palavra vazia caso $t=1$ e $b_{2} \ldots b_{s}$ é a palavra vazia se $s=1$ ).

Note que desse modo $V$ é o conjunto que satisfaz a condição do Lema 3.6, pois dado $A_{\sigma} \in[V]^{\omega}$, $\left(x_{\sigma, n}\right)_{n \geq 1}$ e $\left(y_{\sigma, n}\right)_{n \geq 1}$ são sequências que satisfazem a propriedade exigida. Resta agora provar que de fato $S$ com tal operação é um semigrupo cancelativo.

Vejamos que $\cdot$ é associativa: sejam $u=a_{1} \ldots a_{t}, v=b_{1} \ldots b_{s}$ e $w=c_{1} \ldots c_{r}$ elementos de $S$.

Caso 1: $b_{1}=y_{\sigma, n}$ para algum $\sigma<\mathfrak{c}$ e algum $n \in \mathbb{N}$, e $b_{s}=x_{\tau, m}$ para algum $\tau<\mathfrak{c}$ e algum $m \in \mathbb{N}$. (Note que neste caso $s \geq 2$ pois $b_{1} \neq b_{s}$.)

Caso 1a: $a_{t} \neq x_{\sigma, k}$ para todos $k<n$, e $c_{1} \neq y_{\tau, l}$ para todos $l>m$. Neste caso, temos que ambos $(u \cdot v) \cdot w$ e $u \cdot(v \cdot w)$ são a concatenação de $u, v$ e $w$, portanto iguais.

Caso 1b: $a_{t}=x_{\sigma, k}$ para algum $k<n$, e $c_{1} \neq y_{\tau, l}$ para todos $l>m$. Temos então

$$
\begin{aligned}
(u \cdot v) \cdot w & =\left(a_{1} \ldots a_{t-1} w_{\sigma, k, n} b_{2} \ldots b_{s}\right) \cdot\left(c_{1} \ldots c_{r}\right) \\
& =a_{1} \ldots a_{t-1} w_{\sigma, k, n} b_{2} \ldots b_{s} c_{1} \ldots c_{r} \\
& =\left(a_{1} \ldots a_{t}\right) \cdot\left(b_{1} \ldots b_{s} c_{1} \ldots c_{r}\right) \\
& =u \cdot(v \cdot w) .
\end{aligned}
$$

Caso 1c: $a_{t} \neq x_{\sigma, k}$ para todos $k<n$, e $c_{1}=y_{\tau, l}$ para algum $l>m$. Temos então

$$
\begin{aligned}
(u \cdot v) \cdot w & =\left(a_{1} \ldots a_{t} b_{1} \ldots b_{s}\right) \cdot\left(c_{1} \ldots c_{r}\right) \\
& =a_{1} \ldots a_{t} b_{1} \ldots b_{s-1} w_{\tau, m, l} c_{2} \ldots c_{r} \\
& =\left(a_{1} \ldots a_{t}\right) \cdot\left(b_{1} \ldots b_{s-1} w_{\tau, m, l} c_{2} \ldots c_{r}\right) \\
& =u \cdot(v \cdot w) .
\end{aligned}
$$


Caso 1d: $a_{t}=x_{\sigma, k}$ para algum $k<n$, e $c_{1}=y_{\tau, l}$ para algum $l>m$. Temos então

$$
\begin{aligned}
(u \cdot v) \cdot w & =\left(a_{1} \ldots a_{t-1} w_{\sigma, k, n} b_{2} \ldots b_{s}\right) \cdot\left(c_{1} \ldots c_{r}\right) \\
& =a_{1} \ldots a_{t-1} w_{\sigma, k, n} b_{2} \ldots b_{s-1} w_{\tau, m, l} c_{2} \ldots c_{r} \\
& =\left(a_{1} \ldots a_{t}\right) \cdot\left(b_{1} \ldots b_{s-1} w_{\tau, m, l} c_{2} \ldots c_{r}\right) \\
& =u \cdot(v \cdot w) .
\end{aligned}
$$

(Note que caso $s=2$ a expressão $b_{2} \ldots b_{s-1}$ é vazia, sem prejuízo para a demonstração.)

Caso 2: $b_{1}=y_{\sigma, n}$ para algum $\sigma<\mathfrak{c}$ e algum $n \in \mathbb{N}$, e $b_{s} \neq x_{\tau, m}$ para todos $\tau<\mathfrak{c}$ e $m \in \mathbb{N}$.

Caso 2a: $a_{t} \neq x_{\sigma, k}$ para todos $k<n$. Neste caso, temos que ambos $(u \cdot v) \cdot w$ e $u \cdot(v \cdot w)$ são a concatenação de $u, v$ e $w$, portanto iguais.

Caso 2b: $a_{t}=x_{\sigma, k}$ para algum $k<n$. Mesma situação do caso $1 \mathrm{~b}$.

Caso 3: $b_{1} \neq y_{\sigma, n}$ para todos $\sigma<\mathfrak{c}$ e $n \in \mathbb{N}$ e $b_{s}=x_{\tau, m}$ para algum $\tau<\mathfrak{c}$ e algum $m \in \mathbb{N}$.

Caso 3a: $c_{1} \neq y_{\tau, l}$ para todos $l>m$. Neste caso, temos que ambos $(u \cdot v) \cdot w$ e $u \cdot(v \cdot w)$ são a concatenação de $u, v$ e $w$, portanto iguais.

Caso 3b: $c_{1}=y_{\tau, l}$ para algum $l>m$. Mesma situação do caso $1 \mathrm{c}$.

Caso 4: $b_{1} \neq y_{\sigma, n}$ para todos $\sigma<\mathfrak{c}$ e $n \in \mathbb{N}$ e $b_{s} \neq x_{\tau, m}$ para todos $\tau<\mathfrak{c}$ e $m \in \mathbb{N}$. Neste caso, temos que ambos $(u \cdot v) \cdot w$ e $u \cdot(v \cdot w)$ são a concatenação de $u, v$ e $w$, portanto iguais.

Agora, faremos a prova de que $S$ é cancelativo à direita. A prova de que $S$ é cancelativo à esquerda é similar. Sejam então $u=a_{1} \ldots a_{t}, v=b_{1} \ldots b_{s}$ e $w=c_{1} \ldots c_{r}$ e suponha que $u \cdot w=v \cdot w$. Se $c_{1} \neq y_{\tau, l}$ para todos $\tau<\mathfrak{c}$ e $l \in \mathbb{N}$, então $u \cdot w$ e $v \cdot w$ são concatenações, $\log o u=v$. Suponha então que $c_{1}=y_{\tau, l}$ para algum $\tau<\mathfrak{c}$ e algum $l \in \mathbb{N}$.

Caso a: $a_{t} \neq x_{\tau, n}$ para todos $n<l$ e $b_{s} \neq x_{\tau, m}$ para todos $m<l$. Neste caso, $u \cdot w$ e $v \cdot w$ são concatenações, $\operatorname{logo} u=v$.

Caso b: $a_{t}=x_{\tau, n}$ para algum $n<l$ e $b_{s} \neq x_{\tau, m}$ para todos $m<l$. Temos que $u \cdot w=$ $a_{1} \ldots a_{t-1} w_{\tau, n, l} c_{2} \ldots c_{r}$ e $v \cdot w=b_{1} \ldots b_{s} c_{1} \ldots c_{r}$. Como coincidem termo a termo, temos que $c_{1}=w_{\tau, n, l}$. Absurdo, pois $c_{1}=y_{\tau, l}$.

Caso c: $a_{t} \neq x_{\tau, n}$ para todos $n<l$ e $b_{s}=x_{\tau, m}$ para algum $m<l$. Analogamente ao caso $\mathrm{b}$, temos um absurdo.

Caso d: $a_{t}=x_{\tau, n}$ para algum $n<l$ e $b_{s}=x_{\tau, m}$ para algum $m<l$. Temos que $u \cdot w=$ $a_{1} \ldots a_{t-1} w_{\tau, n, l} c_{2} \ldots c_{r}$ e $v \cdot w=b_{1} \ldots b_{s-1} w_{\tau, m, l} c_{2} \ldots c_{r}$. Como as palavras são iguais, seu 
comprimento é igual, ou seja $(t-1)+1+(r-1)=(s-1)+1+(r-1)$, do que $t=s$. Disso segue que para cada $i<t, a_{i}=b_{i}$; e $w_{\tau, n, l}=w_{\tau, m, l}$, do que $n=m$, e portanto $a_{t}=x_{\tau, n}=x_{\tau, m}=b_{s}$. Assim $a_{1} \ldots a_{t}=b_{1} \ldots b_{s}$, ou seja, $u=v$.

Antes do próximo teorema do artigo, precisaremos de um resultado do livro [1] sobre cancelamento.

Teorema 3.8. Seja $S$ um semigrupo e seja s um elemento cancelável de $S$. Então, para cada $t \in S$ e cada $p \in \beta S$ :

(a) Se $S$ é cancelativo à direita e $s p=t p$, então $s=t$.

(b) Se $S$ é cancelativo à esquerda e ps $=p t$, então $s=t$.

Demonstração. Provaremos (i); (ii) é análogo. A prova será por contraposição.

Suponha que $s \neq t$. Defina $f: \beta S \rightarrow \beta S$ por $f(s u)=t u$ para todo $u \in S$, e $f(v)=s^{2}$ para todo $v \in S \backslash s S$. Note que a função está bem definida, já que sobre $s S$, se $s u=s u^{\prime}$, como $s$ é cancelável, então $u=u^{\prime}$. Além disso, como $S$ é cancelativo à direita, $f$ não possui pontos fixos (já que estamos supondo $s \neq t)$.

Agora, note que as funções contínuas $\tilde{f} \circ \lambda_{s}$ e $\lambda_{t}$ coincidem sobre $S$, logo são iguais, de forma que $\widetilde{f}(s p)=t p$. Usando o Teorema 1.26(a), particione $S$ em três células $A_{1}, A_{2}, A_{3}$ tais que $A_{i} \cap f\left[A_{i}\right]=\varnothing$ para cada $i$. Tome $i$ tal que $A_{i} \in s p$. Pelo Teorema 1.26(e), $f\left[A_{i}\right] \in \tilde{f}(s p)=t p$. Dessa forma, $s p \neq t p$.

Como corolário, se $S$ é cancelativo, então $\beta S$ é cancelável com relação a $S$.

O próximo teorema nos diz que, com uma certa restrição sobre a cardinalidade de $S$, podemos concluir mais do que o Teorema 3.4.

Teorema 3.9. Seja $S$ um semigrupo imersivel em um grupo $G$ e suponha que $|S|=\kappa \geq \omega$ e $\kappa^{\omega}<2^{\mathfrak{c}}$. Seja $\mathcal{V}=\left\{A \in[S]^{\omega}: A^{*} \cap G^{*} G^{*}=\varnothing\right\}$ e defina $T=\bigcup\left\{A^{*}: A \in \mathcal{V}\right\}$. Então:

(a) $T$ é um aberto denso de $S^{*}, T \cap G^{*} G^{*}=\varnothing$, e todo elemento de $T$ é cancelável à direita com relação a $Q=\left\{q \in S^{*}:\|q\| \leq \omega\right\}$ (ou seja, dados $p \in T$ e $q, r \in Q$, se $q p=r p$ então $q=r$ ).

(b) Seja $H$ o subgrupo de $G$ gerado por $S$. Defina uma relação de equivalência $\sim$ em $S$ por $p \sim q$ se e só se existem $a, b \in H$ tais que apb $=q$. Então dados $k, m \in \mathbb{N}, p_{1}, \ldots, p_{m}, q_{1}, \ldots, q_{k} \in T$, se $p_{1} \cdots p_{m} \sim q_{1} \cdots q_{k}$, então $k=m$ e $p_{t} \sim q_{t}$ para cada $t$.

(c) Existe $X \subset T$ tal que $X$ é denso em $S^{*},|X|=2^{\mathfrak{c}}$ e $X$ gera um subsemigrupo livre de $S^{*}$. 
Demonstração. (a): Pela própria definição, $T$ é aberto em $S^{*}$ e disjunto de $G^{*} G^{*}$. Agora, pelo Teorema 3.4, $S^{*} \cap G^{*} G^{*}$ é nunca denso em $S^{*}$, e logo $S^{*} \backslash G^{*} G^{*}$ é denso em $S^{*}$. Dado $V^{*} \subset S^{*} \backslash G^{*} G^{*}$ aberto básico de $S^{*}$, tome $A \subset V$ enumerável. Temos que $A \in \mathcal{V}$, mostrando que $T \cap V^{*} \neq \varnothing$, e portanto $T$ é denso em $S^{*} \backslash G^{*} G^{*}$.

Agora, seja $p \in T$ e suponha $q, r \in Q$ distintos tais que $q p=r p$. Como $\|q\|,\|r\| \leq \omega$, tome $A \in q$, $B \in r$ enumeráveis e disjuntos. Temos então que $q p \in \bar{A} p=\overline{A p}$ e $r p \in \bar{B} p=\overline{B p}$. Pelo item (c) do Teorema 1.26, temos que $A p \cap \bar{B} p \neq \varnothing$ ou $\bar{A} p \cap B p \neq \varnothing$. S.p.g., tome $a \in A$ e $b \in \bar{B}$ tais que $a p=b p$. Pelo Teorema 3.8 acima, caso $b \in B$, então $a=b$; absurdo pois $A \cap B=\varnothing$. Logo $b \in B^{*}$. Temos então que $p=a^{-1} b p$. Como pelo Teorema 1.40, $G^{*}$ é um ideal, $a^{-1} b \in G^{*}$, de forma que $p=a^{-1} b p \in G^{*} G^{*}$. Contradição, pois $p \in T$.

(b): Para provar este, será necessário observar dois fatos:

Primeiramente, notar que $T \subset\left\{p \in S^{*}:\|p\|=\omega\right\}$, pois exigimos $|A|=\omega$ na definição de $\mathcal{V}$.

Segundo, que dados $A \in p$ e $B \in q, A B \in p q$, pois $A \subset\left\{x \in S: x^{-1} A B \in q\right\}$, já que dado $a \in A$, $B \subset a^{-1} A B$. Disso segue, usando que $S$ é cancelativo, que $\left\{p \in S^{*}:\|p\|=\omega\right\}$ é um subsemigrupo de $S^{*}$, e um ideal de $\{p \in \beta S:\|p\| \leq \omega\}$.

É fácil ver que $\sim$ é uma relação de equivalência (basta usar que $H$ é grupo).

Sejam então $k, m \in \mathbb{N}$ e $p_{1}, \ldots, p_{m}, q_{1} \ldots, q_{k} \in T$ tais que $p_{1} \cdots p_{m} \sim q_{1} \cdots q_{k}$. Suponha que a conclusão falhe, e tome $k+m$ minimal entre todos os contraexemplos. Vale que $m>1$ e $k>1$ : se $m=1$ e $k=1$, não é um contraexemplo; se $m=1$ e $k>1$, teríamos, para algum $a, b \in H$, $p_{1}=\left(a^{-1} q_{1} \cdots q_{k-1}\right)\left(q_{k} b^{-1}\right) \in G^{*} G^{*}$, uma contradição; analogamente se $m>1$ e $k=1$.

Tome então $a, b \in H$ tais que $a p_{1} \cdots p_{m} b=q_{1} \cdots q_{k}$. Pelas observações acima, podemos tomar $A, B \subset S$ enumeráveis tais que $A \in p_{1} \cdots p_{m-1}$ e $B \in q_{1} \cdots q_{k-1}$. Temos então que $a \bar{A} p_{m} b \cap \bar{B} q_{k} \neq$ $\varnothing$; novamente pelo Teorema 1.26(c), temos duas opções: podemos tomar (1) $c \in A$ e $d \in \bar{B}$ tais que $a c p_{m} b=d q_{k}$; ou (2) $c \in A^{*}$ e $d \in B$ tais que $a c p_{m} b=d q_{k}$. Suponha que valha (2): teríamos então $q_{k}=\left(d^{-1} a c\right)\left(p_{m} b\right) \in G^{*} G^{*}$, uma contradição. Logo vale (1). Caso $d \in B^{*}$, teríamos que $p_{m}=\left((a c)^{-1} d\right)\left(q_{k} b^{-1}\right) \in G^{*} G^{*}$, contradição. Logo $d \in B$, e assim $d^{-1} a c p_{m} b=q_{k}$, ou seja, $p_{m} \sim q_{k}$. Além disso, $p_{m} b=(a c)^{-1} d q_{k}$, de forma que $a p_{1} \cdots p_{m-1}(a c)^{-1} d q_{k}=q_{1} \cdots q_{k}$. Pelo item (a), podemos cancelar $q_{k}$ de ambos os lados e obter que $a p_{1} \cdots p_{m-1}(a c)^{-1} d=q_{1} \cdots q_{k-1}$, ou seja, $p_{1} \cdots p_{m-1} \sim q_{1} \cdots q_{k-1}$. Pela minimalidade de $k+m$, obtemos que $k-1=m-1$ e $p_{t} \sim q_{t}$ para todo $t \leq m$.

(c): Denote por $[x]$ a classe de equivalência de $x \in S^{*}$. Note que $[x]=H x H$, de forma que $|[x]|=|H|=|S|=\kappa<2^{\mathfrak{c}}$. Defina $\lambda=|\mathcal{V}| . \mathcal{V} \subset[S]^{\omega}, \operatorname{logo} \lambda \leq \kappa^{\omega}<2^{\mathfrak{c}}$. Seja $\mathcal{V}=\left\{A_{\sigma}: \sigma<\lambda\right\}$ uma enumeração de $\mathcal{V}$. Dado $\sigma<\lambda$, como $\left|A_{\sigma}\right|=\omega,\left|A_{\sigma}{ }^{*}\right|=2^{\mathfrak{c}}$; disso segue que $I_{\sigma}=\left\{[x]: x \in A_{\sigma}{ }^{*}\right\}$ 
tem cardinalidade $2^{\mathfrak{c}}$, pois $A_{\sigma}{ }^{*}=\bigcup_{[x] \in I_{\sigma}} A_{\sigma}{ }^{*} \cap[x]$. Dessa forma, podemos indutivamente escolher, para $\sigma<\lambda, x_{\sigma} \in A_{\sigma}{ }^{*}$ tal que se $\sigma \neq \tau, x_{\sigma} \nsim x_{\tau}$ (em cada passo tome $x_{\sigma} \in \bigcup\left\{I_{\sigma} \backslash\left\{\left[x_{\tau}\right]: \tau<\sigma\right\}\right\}$ ). Assim, defina $\mathcal{S}=I_{0} \backslash\left\{\left[x_{\sigma}\right]: \sigma<\lambda\right\}=\left\{[x]: x \in A_{0}{ }^{*}\right\} \backslash\left\{\left[x_{\sigma}\right]: \sigma<\lambda\right\}$. Tome $Y \subset A_{0}{ }^{*}$ tal que $\mathcal{S}=\{[y]: y \in Y\}$ e dados $y, z \in Y$ distintos, $y \nsim z-$ ou seja, $Y$ é um conjunto escolha de $\left\{[x] \cap A_{0}{ }^{*}:[x] \in \mathcal{S}\right\}$. Por fim, defina $X=Y \cup\left\{x_{\sigma}: \sigma<\lambda\right\}$. Em primeiro lugar, note que $|Y|=|\mathcal{S}|=2^{\mathfrak{c}}$, pois $\left|I_{0}\right|=2^{c}$. Além disso, $X$ possui no máximo um representante de cada classe de equivalência; em particular $Y \cap\left\{x_{\sigma}: \sigma<\lambda\right\}=\varnothing$, e portanto $|X|=|Y|+\lambda=2^{\mathfrak{c}}$.

Vejamos agora que $X$ gera um subsemigrupo livre. Suponha $p_{1}, \ldots, p_{m}, q_{1} \ldots, q_{k} \in X$ tais que $p_{1} \cdots p_{m}=q_{1} \cdots q_{k}$. Note que $X \subset T$, logo o item (b) nos diz que $k=m$ e $p_{t} \sim q_{t}$ para cada $t$. Porém, como $X$ possui no máximo um representante de cada classe, isso implica que $p_{t}=q_{t}$. Ou seja, $X$ de fato gera um subsemigrupo livre.

Por fim, para ver que $X$ é denso em $S^{*}$, seja $V \subset S$ infinito e tome $D \subset V$ enumerável. Tome $q \in D^{*} \backslash \mathrm{cl} G^{*} G^{*}$, e tome $B \in q$ tal que $B^{*} \cap G^{*} G^{*}=\varnothing$. Considere $A=B \cap D$; temos que $A \in \mathcal{V}$, $\operatorname{logo} A=A_{\sigma}$ para algum $\sigma<\lambda$; deste modo, $x_{\sigma} \in X$ e $x_{\sigma} \in A_{\sigma}{ }^{*} \subset D^{*} \subset V^{*}$, de modo que $V^{*} \cap X \neq \varnothing$.

Os próximos três teoremas lidam com a densidade dos produtos em $\mathcal{U}_{\kappa}$, e por isso veremos sob que condições temos $S^{*} \mathcal{U}_{\kappa} \subset \mathcal{U}_{\kappa}$. O que segue é o Lema 6.34 .3 do livro [2].

Lema 3.10. Seja $S$ um semigrupo infinito e $\kappa=|S|$. Se $S$ é fraquissimamente cancelativo à esquerda, então $\mathcal{U}_{\kappa}(S)$ é um ideal à esquerda de $\beta S$. Se além disso, $S$ for fraquissimamente cancelativo à direita, então $\mathcal{U}_{\kappa}(S)$ é um ideal de $\beta S$.

Demonstração. Sejam $p \in \beta S$ e $q \in \mathcal{U}_{\kappa}$, e suponha que $p \cdot q \notin \mathcal{U}_{\kappa}$. Tome $A \in p \cdot q$ tal que $|A|<\kappa$. Vale que $\left\{x \in S: x^{-1} A \in q\right\} \in p$; tome $x$ tal que $x^{-1} A \in q$. Temos então: $x^{-1} A=\bigcup_{a \in A}\{y \in S$ : $x y=a\}$. Como $|A|<\kappa$, e $S$ é fraquissimamente cancelativo à esquerda, segue que $\left|x^{-1} A\right|<\kappa$. Absurdo, pois $x^{-1} A \in q \in \mathcal{U}_{\kappa}$.

Agora, suponha que $q \cdot p \notin \mathcal{U}_{\kappa}$ e tome $A \in q \cdot p$ tal que $|A|<\kappa$. Caso $p \in \mathcal{U}_{\kappa}$, contradiz o que acabamos de provar. Então $\|p\|<\kappa$, logo tome $B \in p$ tal que $|B|<\kappa$. Temos, pois: $C=\{x \in S$ : $\left.x^{-1} A \in p\right\} \in q$. Note que $|C|=\kappa$. Agora, afirmamos que $C \subset \bigcup_{b \in B} \bigcup_{a \in A}\{x \in S: x a=b\}$, do que segue que $|C|<\kappa$. Para comprovar a afirmação, seja $x \in C$; tome $b \in B \cap x^{-1} A$, e tome $a \in A$ tal que $x a=b$; segue a afirmação.

Observe que o próximo teorema é uma de extensão do caso $S$ enumerável (3.3), pois $\mathcal{U}_{\omega}=S^{*}$, e inclusive possui técnica de demonstração similar.

Teorema 3.11. Seja $S$ um semigrupo cancelativo à direita e fraquissimamente cancelativo à esquerda com $|S|=\kappa$. Então $S^{*} \mathcal{U}_{\kappa}$ é nunca-denso em $\mathcal{U}_{\kappa}$. 
Demonstração. Enumere $S=\left\{s_{\alpha}: \alpha<\kappa\right\}$. Suponha, por absurdo, que existe $V \in[S]^{\kappa}$ tal que $\bar{V} \cap \mathcal{U}_{\kappa} \subset \operatorname{cl} S^{*} \mathcal{U}_{\kappa}$.

Tome $v_{0} \in V$ qualquer. Recursivamente, seja $0<\delta<\kappa$ e suponha que já escolhemos $v_{\sigma}$ para $\sigma<\delta$ de forma que

(1) se $\alpha<\sigma<\delta$, então $v_{\alpha}<v_{\sigma}$; e

(2) se $\alpha<\sigma<\tau<\delta, \mu<\tau$, e $x \in S$, então $s_{\alpha} x \neq v_{\sigma}$ ou $s_{\mu} x \neq v_{\tau}$.

Para cada $\alpha<\sigma<\delta$, defina $C_{\alpha, \sigma}=\left\{x \in S: s_{\alpha} x=v_{\sigma}\right\}$; considere $B=\bigcup_{\alpha<\sigma<\delta} C_{\alpha, \sigma}$. Como $B$ é uma união de menos do que $\kappa$ conjuntos solução à esquerda, então $|B|<\kappa$; dessa forma, $\mid\left\{s_{\mu} x: \mu<\delta\right.$ e $\left.x \in B\right\} \mid<\kappa$. Isso nos permite tomar $v_{\delta} \in V \backslash\left(\left\{v_{\sigma}: \sigma<\delta\right\} \cup\left\{s_{\mu} x: \mu<\delta\right.\right.$ e $\left.\left.x \in B\right\}\right)$. Claramente a condição (1) permanece satisfeita. Para (2), suponha $\alpha<\sigma<\delta, \mu<\delta$, e $x \in S$ tais que $s_{\alpha} x=v_{\sigma}$ e $s_{\mu} x=v_{\delta}$. Teríamos que $x \in C_{\alpha, \sigma} \subset B$, de forma que $v_{\delta} \in\left\{s_{\mu} x: \mu<\delta\right.$ e $\left.x \in B\right\}$, uma contradição.

Defina então $A=\left\{v_{\sigma}: \sigma<\kappa\right\}$ e, para cada $\alpha<\kappa, A_{\alpha}=\left\{v_{\sigma}: \alpha<\sigma<\kappa\right\}$. Necessitaremos do seguinte fato: dados $s \in S$ e $q \in \mathcal{U}_{\kappa}$, se $s^{-1} A \in q$, então para todo $\alpha<\kappa, s^{-1} A_{\alpha} \in q$. Suponha que a conclusão falhe e tome $\alpha<\kappa$ tal que $s^{-1} A_{\alpha} \notin q$. Temos que $s^{-1} A \backslash s^{-1} A_{\alpha}=s^{-1}\left(A \backslash A_{\alpha}\right) \in q$. Note que $s^{-1}\left(A \backslash A_{\alpha}\right)=\bigcup_{\sigma \leq \alpha}\left\{y \in S: s y=v_{\sigma}\right\}$; esse é uma união de menos do que $\kappa$ conjuntos solução à esquerda, logo possui cardinalidade menor do que $\kappa$. Contradição pois $q \in \mathcal{U}_{\kappa}$.

Agora, como $A \subset V, \bar{A} \cap \mathcal{U}_{\kappa} \subset \operatorname{cl} S^{*} \mathcal{U}_{\kappa}$, logo $\bar{A} \cap \mathcal{U}_{\kappa} \cap S^{*} \mathcal{U}_{\kappa} \neq \varnothing$. Portanto tome $p \in S^{*}$ e $q \in \mathcal{U}_{\kappa}$ tais que $A \in p \cdot q$. Temos que $\left\{s \in S: s^{-1} A \in q\right\} \in p$, logo tome $\alpha, \mu<\kappa$ distintos tais que $s_{\alpha}{ }^{-1} A, s_{\mu}{ }^{-1} A \in q$. Tome $x \in s_{\alpha}{ }^{-1} A_{\alpha} \cap s_{\mu}{ }^{-1} A_{\mu}$. Tome então $\sigma, \delta<\kappa$ tais que $\alpha<\sigma, \mu<\delta$ e $s_{\alpha} x=v_{\sigma}$ e $s_{\mu} x=v_{\delta}$. Como $S$ é cancelativo à direita e $s_{\alpha} \neq s_{\mu}$, temos que $\sigma \neq \delta$. Sem perda de generalidade, $\sigma<\delta$; isso viola a construção de $v_{\delta}$, contradição.

Os autores do artigo apontam que, ao se perscrutar as últimas três linhas da demonstração acima, poder-se-ia exigir menos do que o cancelamento à direita: se exigíssemos apenas que dados $a, b \in S$ distintos, $|\{y \in S: a y=b y\}|<\kappa$, então tomar-se-ia $x \in\left(s_{\alpha}{ }^{-1} A_{\alpha} \cap s_{\mu}{ }^{-1} A_{\mu}\right) \backslash\{y \in S:$ $\left.s_{\alpha} y=s_{\mu} y\right\}$ e a demonstração seguiria. Porém, apontam em seguida os autores, isto não é um enfraquecimento das hipóteses, pois isto implica que $S$ é cancelativo à direita, do seguinte modo: suponha $a, b \in S$ distintos e $c \in S$ tais que $a c=b c$. Então $c S \subset\{y \in S: a y=b y\}$ e portanto $|c S|<\kappa$. Porém, $S=\bigcup_{d \in c S}\{x \in S: c x=d\}$, uma união de menos do que $\kappa$ conjuntos solução à esquerda, de modo que $|S|<\kappa$, contradição.

Lema 3.12. Seja $S$ um semigrupo infinito, fracamente cancelativo à esquerda e fraquissimamente cancelativo à direita, com $|S|=\kappa$. Enumere $S=\left\{s_{\alpha}: \alpha<\kappa\right\}$ e seja $V=\left\{q \in S^{*}\right.$ : existe $\delta<\kappa$ tal que $\left.\left\{s_{\alpha}: \alpha<\delta\right\} \in q\right\}$. Então $(\beta S) V \cap \mathcal{U}_{\kappa}$ é nunca-denso em $U_{\kappa}$. 
Demonstração. Suponha por absurdo que existe $C \in[S]^{\kappa}$ tal que $\bar{C} \cap \mathcal{U}_{\kappa} \subset \operatorname{cl}((\beta S) V)$. Usando técnicas análogas ao do teorema anterior - apenas "espelhadas" - obtemos uma $\kappa$-sequência $\left(t_{\alpha}\right)_{\alpha<\kappa}$ em $C$ injetora tal que dados $x \in S, \gamma<\sigma<\alpha$, e $\mu<\alpha$, vale que $x s_{\gamma} \neq t_{\sigma}$ ou $x s_{\mu} \neq t_{\alpha}$. (Precisamos apenas usar que $|C|=\kappa$ e $S$ é fraquissimamente cancelativo à direita.)

Defina $B=\left\{t_{\alpha}: \alpha<\kappa\right\}$. Como $\bar{B} \cap \mathcal{U}_{\kappa} \cap(\beta S) V \neq \varnothing$, tome $r \in \beta S$ e $q \in V$ tais que $r \cdot q \in \bar{B} \cap \mathcal{U}_{\kappa}$. Como $q \in V$, tome $\delta<\kappa$ tal que $\left\{s_{\alpha}: \alpha<\delta\right\} \in q$, e considere $H=\left\{t_{\alpha}: \alpha>\delta\right\}$. Como $B \in r \cdot q \in \mathcal{U}_{\kappa}$, então $H \in r \cdot q$, de forma que $\left\{x \in S: x^{-1} H \in q\right\} \in r$, logo tome um tal $x$. Defina $W=x^{-1} H \cap\left\{s_{\alpha}: \alpha<\delta\right\}$; note que $W \in q$, e logo é infinito.

Afirmação: $|x W|=1$. Sejam $\gamma, \mu<\delta$ tais que $s_{\gamma}, s_{\mu} \in W$. Temos $x s_{\gamma}, x s_{\mu} \in H$, logo tome $\sigma, \alpha>\delta$ tais que $x s_{\gamma}=t_{\sigma}$ e $x s_{\mu}=t_{\alpha}$. Caso $\sigma \neq \alpha$, então $\sigma<\alpha$ ou $\alpha<\sigma$, contradizendo a escolha da $\kappa$-sequência. Portanto $\alpha=\sigma, \log 0 x s_{\gamma}=x s_{\mu}$.

Porém, $W$ ser infinito e $x W$ ser unitário contradiz a hipótese de $S$ ser fracamente cancelativo à esquerda.

Teorema 3.13. Seja $S$ um semigrupo infinito, cancelativo à direita, e fracamente cancelativo à esquerda, com $|S|=\kappa$. Suponha que $\kappa$ é regular. Então $S^{*} S^{*} \cap \mathcal{U}_{\kappa}$ é nunca-denso em $U_{\kappa}$.

Demonstração. Pelo Teorema 3.11, $S^{*} \mathcal{U}_{\kappa}$ é nunca-denso em $\mathcal{U}_{\kappa}$. Resta ver então que $S^{*}\left(S^{*} \backslash \mathcal{U}_{\kappa}\right) \cap \mathcal{U}_{\kappa}$ é nunca-denso. Enumere $S=\left\{s_{\alpha}: \alpha<\kappa\right\}$ e seja $V=\left\{q \in S^{*}\right.$ : existe $\delta<\kappa$ tal que $\left\{s_{\alpha}: \alpha<\delta\right\} \in$ $q$ \}. Pelo Lema acima, $(\beta S) V \cap \mathcal{U}_{\kappa}$ é nunca-denso em $\mathcal{U}_{\kappa}$; em particular $S^{*} V \cap \mathcal{U}_{\kappa}$ é nunca-denso. Basta ver então que $S^{*} \backslash \mathcal{U}_{\kappa} \subset V$. Seja $r \in S^{*} \backslash \mathcal{U}_{\kappa}$ então. Tome $A \in r$ tal que $|A|<\kappa$. Como $\kappa$ é regular, então existe $\delta<\kappa$ tal que $A \subset\left\{s_{\alpha}: \alpha<\delta\right\}$; esse $\delta$ atesta que $r \in V$.

Para o próximo teorema - o último nesta seção do artigo - precisaremos de um resultado encontrado no Teorema 7.35 do livro [1]. Apresentaremos a prova deste resultado posteriormente.

O próximo teorema mostra a grande maioria das técnicas utilizadas nesta seção. Ele traduz a ideia de que as propriedades algébricas de $S$ influenciam nas propriedades topológicas-e-algébricas de $S^{*}$, sendo que cada particularidade destas têm seu papel bem delineado.

Teorema 3.14. Seja S um semigrupo infinito, cancelativo à direita, e fraquissimamente cancelativo à esquerda, com $|S|=\kappa$. Seja $\mathcal{C}$ uma coleção de no máximo $\kappa$ subconjuntos de $S$ tal que $\mathcal{C}$ possui a $\kappa$-p.i.f. Então existe uma $\kappa$-sequência injetora $\left(t_{\alpha}\right)_{\alpha<\kappa}$ em $S$ tal que, definindo $B=\left\{t_{\alpha}: \alpha<\kappa\right\}$, valem as seguintes asserções:

(1) $\bar{B} \cap \mathcal{U}_{\kappa} \cap S^{*} \mathcal{U}_{\kappa}=\varnothing$.

(2) Se $p, q \in \bar{B} \cap \mathcal{U}_{\kappa}$ são distintos, então $\beta S \cdot p \cap \beta S \cdot q=\varnothing$.

(3) Se $p \in \bar{B} \cap \mathcal{U}_{\kappa}$, então $p$ é cancelável à direita em $\beta S$. 
(4) $\left|\bar{B} \cap \mathcal{U}_{\kappa}\right|=2^{2^{\kappa}}$ e $\bar{B} \cap \mathcal{U}_{\kappa}$ gera um semigrupo livre em $\mathcal{U}_{\kappa}$.

(5) Seja $T=\bigcap_{\alpha<\kappa} \overline{F P\left(\left(t_{\sigma}\right)_{\alpha<\sigma<\kappa}\right)}$. Então $T$ é um subsemigrupo compacto de $\beta S$ com a propriedade de que todo grupo maximal de $K(T)$ contém uma cópia do grupo livre sobre $2^{2^{\kappa}}$ geradores. Em particular, $\overline{F P\left(\left(t_{\alpha}\right)_{\alpha<\kappa}\right)}$ possui uma cópia do grupo livre sobre $2^{2^{\kappa}}$ geradores.

(6) Se $\kappa$ é regular e $S$ é fracamente cancelativo à esquerda, então $\bar{B} \cap \mathcal{U}_{\kappa} \cap S^{*} S^{*}=\varnothing$.

Além disso, existe $P \subset \bar{B} \cap \mathcal{U}_{\kappa}$ tal que $|P|=2^{2^{\kappa}}$ e, para todo $p \in P, \mathcal{C} \subset p$.

Demonstração. A ideia da prova é obter uma $\kappa$-sequência, como em teoremas anteriores, que possui propriedades algébricas suficientes para garantir os itens acima, e de forma que se possa construí-la por recursão transfinita.

Enumere $S=\left\{s_{\alpha}: \alpha<\kappa\right\}$. Podemos supor que $\mathcal{C}$ é fechado para interseções finitas (ou seja acrescê-lo de suas interseções finitas, sem alterar a cardinalidade). Seja $\lambda=|\mathcal{C}|$, enumere $\mathcal{C}=\left\{C_{\alpha}: \alpha<\lambda\right\}$, e tome $f: \kappa \rightarrow \kappa \times \lambda$ bijeção. Fixe um $a \in S$ e defina $A=\{s \in S: a s=a\} . A$ é um conjunto solução à esquerda, $\log |A|<\kappa$. Tome $t_{0} \in C_{\pi_{2}(f(0))} \backslash A$, em que $\pi_{2}$ é a projeção de $\kappa \times \lambda$ sobre $\lambda$.

Agora, seja $0<\alpha<\kappa$ e suponha que já escolhemos $\left(t_{\delta}\right)_{\delta<\alpha}$ de forma que

(a) se $\delta<\alpha$, então $t_{\delta} \notin F P\left(\left(t_{\gamma}\right)_{\gamma<\delta}\right)$;

(b) se $\delta<\alpha$, então $t_{\delta} \in C_{\pi_{2}(f(\delta))}$;

(c) se $\gamma<\delta<\alpha$ e $\mu<\sigma<\delta$, então $s_{\gamma} t_{\delta} \neq s_{\mu} t_{\sigma}$;

(d) se $\gamma<\sigma<\delta<\alpha, \mu<\delta$, e $x \in S$, então $s_{\gamma} x \neq t_{\sigma}$ ou $s_{\mu} x \neq t_{\delta}$;

(e) se $\delta<\alpha$ e $u, v \in F P\left(\left(t_{\gamma}\right)_{\gamma<\delta}\right)$, então $u \neq v t_{\delta}$;

(f) se $\delta<\alpha, u, v \in F P\left(\left(t_{\gamma}\right)_{\gamma<\delta}\right)$, e $u \neq v$, então $u t_{\delta} \neq v t_{\delta}$;

(g) se $\delta<\alpha$ e $u \in F P\left(\left(t_{\gamma}\right)_{\gamma<\delta}\right)$, então $u t_{\delta} \neq t_{\delta}$;

(h) $F P\left(\left(t_{\delta}\right)_{\delta<\alpha}\right) \cap A=\varnothing ; \mathrm{e}$

(i) se $\kappa$ é regular, $S$ é fracamente cancelativo à esquerda, $x \in S, \gamma<\sigma<\delta<\alpha$, e $\mu<\delta$, então $x s_{\gamma} \neq t_{\sigma}$ ou $x s_{\mu} \neq t_{\delta}$.

Vale comentar que cada um desses itens tem um papel específico a cumprir: por exemplo, o item (c) tem seu papel nos itens (2) e (3), e os itens (a), (e) e (g) são necessários para se provar (5). As passagens têm embutida nelas a ideia de que as propriedades da $\kappa$-sequência passam via limites ao fecho de sua imagem - com as devidas restrições da continuidade lateral da operação. 
Note que as hipóteses estão de fato todas satisfeitas para $\alpha=1$, e todas exceto (b) e (h) por vacuidade.

Para cada $\mu<\sigma<\alpha$ e $\gamma<\alpha$, seja $A_{\gamma, \mu, \sigma}=\left\{x \in S: s_{\gamma} x=s_{\mu} t_{\sigma}\right\}$. Cada $A_{\gamma, \mu, \sigma}$ é um conjunto solução à esquerda, logo $\left|\bigcup_{\gamma<\alpha} \bigcup_{\mu<\sigma<\alpha} A_{\gamma, \mu, \sigma}\right|<\kappa$.

Para cada $\gamma<\sigma<\alpha$, seja $F_{\gamma, \sigma}=\left\{x \in S: s_{\gamma} x=t_{\sigma}\right\}$. Cada $F_{\gamma, \sigma}$ é um conjunto solução à esquerda, $\operatorname{logo}\left|\bigcup_{\gamma<\sigma<\alpha} F_{\gamma, \sigma}\right|<\kappa$, e portanto $\mid\left\{s_{\mu} x: \mu<\alpha\right.$ e $x \in \bigcup_{\gamma<\sigma<\alpha} F_{\gamma, \sigma} \mid<\kappa$.

Agora, seja $V=F P\left(\left(t_{\delta}\right)_{\delta<\alpha}\right)$. $|V|<\kappa$. Pela hipótese (h), dado $u \in V$, au $\neq a$. Usando mais vezes que $S$ é fraquissimamente cancelativo à esquerda, temos que $\left|\bigcup_{u \in V}\{x \in S: a u x=a\}\right|<\kappa \mathrm{e}$ $\left|\bigcup_{u, v \in V}\{x \in S: v x=u\}\right|<\kappa$.

E se $\kappa$ for regular e $S$ for fracamente cancelativo à esquerda: defina $D=\bigcup_{\gamma<\sigma<\alpha}\left\{x \in S: x s_{\gamma}=t_{\sigma}\right\}$. Como $S$ é cancelativo à direita, $D$ é a união de conjuntos vazios ou unitários, e portanto $|D|<\kappa$, e $\log \mid\left\{x s_{\mu}: \mu<\alpha\right.$ e $\left.x \in D\right\} \mid<\kappa$.

Assim, podemos escolher $t_{\alpha} \in C_{\pi_{2}(f(\alpha))}$ de forma que $t_{\alpha}$ não pertença a $\bigcup_{\gamma<\alpha} \bigcup_{\mu<\sigma<\alpha} A_{\gamma, \sigma, \mu} \cup V \cup$ $A \cup\left\{s_{\mu} x: \mu<\alpha\right.$ e $\left.x \in \bigcup_{\gamma<\sigma<\alpha} F_{\gamma, \sigma}\right\} \cup \bigcup_{u \in V}\{x \in S: a u x=a\} \cup \bigcup_{u, v \in V}\{x \in S: v x=u\}$. Além disso, se $\kappa$ for regular e $S$ for fracamente cancelativo à direita, exija também que $t_{\alpha} \notin\left\{x s_{\mu}: \mu<\alpha\right.$ e $x \in D\}$.

Como $t_{\alpha} \notin V=F P\left(\left(t_{\delta}\right)_{\delta<\alpha}\right)$, temos que a hipótese (a) é satisfeita e a sequência permanece injetora. Como $t_{\alpha} \in C_{\pi_{2}(f(\alpha))}$, a hipótese (b) está satisfeita.

Como $t_{\alpha} \notin \bigcup_{\gamma<\alpha} \bigcup_{\mu<\sigma<\alpha} A_{\gamma, \sigma, \mu}$, a hipótese (c) está satisfeita.

Como $t_{\alpha} \notin\left\{s_{\mu} x: \mu<\alpha\right.$ e $\left.x \in \bigcup_{\gamma<\sigma<\alpha} F_{\gamma, \sigma}\right\}$, a hipótese (d) está satisfeita.

Como $t_{\alpha} \notin \bigcup_{u, v \in V}\{x \in S: v x=u\}$, a hipótese (e) está satisfeita.

A hipótese (f) segue da cancelatividade à direita de $S$.

Para verificar (g), lembre que dado $u \in V, a u \neq a, \operatorname{logo} a u t_{\alpha} \neq a t_{\alpha}$, e portanto $u t_{\alpha} \neq t_{\alpha}$.

Para verificar (h), note que $t_{\alpha} \notin A$ e $t_{\alpha} \notin \bigcup_{u \in V}\{x \in S: a u x=a\}$, logo dado $u \in V$, aut $t_{\alpha} \neq a$, ou seja, $u t_{\alpha} \notin A$.

E se $\kappa$ for regular e $S$ for fracamente cancelativo à direita, $t_{\alpha} \notin\left\{x s_{\mu}: \mu<\alpha\right.$ e $\left.x \in D\right\}$, logo a hipótese (i) está satisfeita.

Feita a indução, defina $B=\left\{t_{\alpha}: \alpha<\kappa\right\}$ e, para cada $\gamma<\kappa, B_{\gamma}=\left\{t_{\alpha}: \gamma<\alpha<\kappa\right\}$. Verifiquemos agora as asserções (1)-(6).

(1): Seja $p \in \bar{B} \cap \mathcal{U}_{\kappa}$ e suponha $q \in S^{*}$ e $r \in \mathcal{U}_{\kappa}$ tal que $p=q \cdot r . B \in q \cdot r$, ou seja, $\{x \in S$ : $\left.x^{-1} B \in r\right\} \in q$, logo tome $\gamma<\mu<\kappa$ tais que $s_{\gamma}{ }^{-1} B, s_{\mu}{ }^{-1} B \in r$. Note que $s_{\gamma}{ }^{-1} B_{\gamma} \in r$, pois $s_{\gamma}{ }^{-1} B \backslash s_{\gamma}{ }^{-1} B_{\gamma}=\bigcup_{\sigma \leq \gamma}\left\{y \in S: s_{\gamma} y=t_{\sigma}\right\}$ é uma união de menos do que $\kappa$ conjuntos solução à esquerda e $r \in \mathcal{U}_{\kappa}$. Analogamente $s_{\mu}{ }^{-1} B_{\mu} \in r$, logo tome $x \in s_{\gamma}{ }^{-1} B_{\gamma} \cap s_{\mu}{ }^{-1} B_{\mu}$. Tome $\sigma>\gamma \mathrm{e}$ $\delta>\mu$ tais que $s_{\gamma} x=t_{\sigma}$ e $s_{\mu} x=t_{\delta}$. Como $S$ é cancelativo à direita, vale que $\sigma \neq \delta$, violando a hipótese (d). 
(2): Sejam $p, q \in \bar{B} \cap \mathcal{U}_{\kappa}$ distintos. Tome $F, G \in[B]^{\kappa}$ tais que $F \cap G=\varnothing, F \in p$ e $G \in q$. Suponha $u, v \in \beta S$ tais que $u p=v q$, e $E=\left\{s_{\gamma} t_{\alpha}: \gamma<\alpha<\kappa\right.$ e $\left.\alpha \in F\right\}$. Vale que $E \in u p$, ou seja, $\left\{x \in S: x^{-1} E \in p\right\} \in u$, pois dado $\gamma<\kappa,\left\{t_{\alpha} \in F: \gamma<\alpha<\kappa\right\} \subset s_{\gamma}{ }^{-1} E$, e $\left\{t_{\alpha} \in F: \gamma<\alpha<\kappa\right\} \in p$. Analogamente, $\left\{s_{\mu} t_{\sigma}: \mu<\sigma<\kappa\right.$ e $\left.t_{\sigma} \in G\right\} \in v q$. Assim, podemos tomar $\gamma<\alpha<\kappa$ e $\mu<\sigma<\kappa$ de forma que $t_{\alpha} \in F, t_{\sigma} \in G$, e $s_{\gamma} t_{\alpha}=s_{\mu} t_{\sigma}$. Como $F \cap G=\varnothing$, $\alpha \neq \sigma$, contradizendo a hipótese (c).

(3): Seja $p \in \bar{B} \cap \mathcal{U}_{\kappa}$ e suponha $u, v \in \beta S$ tais que $u p=v p$. Tome $F \in u$ e $G \in v$ disjuntos. Defina $E=\left\{s_{\gamma} t_{\alpha}: \gamma<\alpha<\kappa\right.$ e $\left.s_{\gamma} \in F\right\}$. Note que, dado $s_{\gamma} \in F,\left\{t_{\alpha}: \gamma<\alpha<\kappa\right\} \in p$ e $\left\{t_{\alpha}: \gamma<\alpha<\kappa\right\} \subset s_{\gamma}{ }^{-1} E$, de forma que $E \in$ up. Analogamente, $\left\{s_{\mu} t_{\sigma}: \mu<\sigma<\kappa\right.$ e $\left.s_{\mu} \in G\right\} \in v p$. Portanto, exatamente como acima, temos uma contradição da hipótese (c).

(4): Como $|B|=\kappa$, o Teorema 1.26(d) nos diz que $\bar{B} \cap \mathcal{U}_{\kappa}(S)$ (que pode ser identificado com $\left.\mathcal{U}_{\kappa}(B)\right)$ tem cardinalidade $2^{2^{\kappa}}$. Agora, para ver que gera um subsemigrupo livre, provaremos que dados $p_{1}, \ldots, p_{m}, q_{1} \ldots, q_{k} \in \bar{B} \cap \mathcal{U}_{\kappa}$, se $p_{1} \cdots p_{m}=q_{1} \cdots q_{k}$, então $k=m$ e $p_{i}=q_{i}$ para cada $i \leq m$. Suponha que não valha, e pegue um contraexemplo tal que $m+k$ é mínimo. Não pode ser que $m+k=2$, pois nesse caso $m=k=1$ e não temos contraexemplo; e por (1), não se pode ter que $m+k=3\left(p_{1}=q_{1} \cdot q_{2}\right.$ ou $\left.p_{1} \cdot p_{2}=q_{1}\right)$. Portanto $m, k>1$. Por $(2)$, temos que $p_{m}=q_{k} ; \operatorname{logo}$, por (3), podemos cancelar e obter $p_{1} \cdots p_{m-1}=q_{1} \cdots q_{k-1}$, do que segue $m-1=k-1$ e $p_{i}=q_{i}$ para cada $i \leq m$.

(5): Pelo Teorema 7.35 do livro [1], basta ver que $\left(t_{\alpha}\right)_{\alpha<\kappa}$ possui produtos finitos distintos. Suponha então $F, G \in \mathcal{P}_{f}(\kappa)$ distintos e tais que $\prod_{\alpha \in F} t_{\alpha}=\prod_{\alpha \in G} t_{\alpha}$, tais que $|F \cup G|$ seja mínimo. Sem perda de generalidade, $\max F \leq \max G=\sigma$. Suponha, primeiramente, que $\max F<\max G$ : caso $G=\{\sigma\}$, teríamos uma violação de (a), e caso $|G| \geq 2$ teríamos uma violação de (e). Então max $F=\max G$, e $\operatorname{logo} \sigma \in F . F$ e $G$ não podem ser ambos unitários pois são distintos. Caso um deles fosse unitário, teríamos uma violação de (g). Logo $|F|,|G| \geq 2$ com $\max F=\max G=\sigma$; podemos cancelar à direita e obter $\prod_{\alpha \in F \backslash\{\sigma\}} t_{\alpha}=\prod_{\alpha \in G \backslash\{\sigma\}}$, do que, pela minimalidade de $|F \cup G|$, $F \backslash\{\sigma\}=G \backslash\{\sigma\}$. Isso implica que $F=G$, contradição.

(6): Suponha que $\kappa$ seja regular e $S$ seja fracamente cancelativo à esquerda. Suponha $q, r \in S^{*}$ tais que $r \cdot q \in \bar{B} \cap \mathcal{U}_{\kappa}$. Pelo item (1), $q \in S^{*} \backslash \mathcal{U}_{\kappa}$. Como $\kappa$ é regular, temos então que existe $\delta<\alpha$ tal que $\left\{s_{\alpha}: \alpha<\delta\right\} \in q$. Deste ponto, obtemos uma contradição exatamente como no final do Teorema 3.12 - usando (a),(i) e que $S$ é fracamente cancelativo à esquerda.

Por fim, considere $\mathcal{B}=\left\{B \cap C_{\alpha}: \alpha<\lambda\right\}$. Vale que $\mathcal{B}$ possui a $\kappa$-p.i.f., pois: dado $F \in \mathcal{P}_{f}(\lambda)$, como $\mathcal{C}$ é fechado para interseções finitas, tome $\gamma<\lambda$ tal que $C_{\gamma}=\bigcap_{\alpha \in F} C_{\alpha}$. Vale que $\left\{t_{\sigma}: \pi_{2}(f(\sigma))=\right.$ $\gamma\} \subset B \cap C_{\gamma}$, e este conjunto tem cardinalidade $\kappa$ pois $\left|\left(\pi_{2} \circ f\right)^{-1}[\{\gamma\}]\right|=\kappa$. Deste modo, basta definir $P=\left\{p \in \mathcal{U}_{\kappa}: \mathcal{B} \subset p\right\}$, e notar que o Teorema 1.26(d) nos garante que $|P|=2^{2^{\kappa}}$.

Agora veremos como se prova o Teorema 7.35 do livro [1]. Começaremos pelo Teorema 1.23 do 
mesmo livro.

Teorema 3.15. Sejam A um conjunto, $G$ o grupo livre gerado por $A$, e $g \in G \backslash\{\varnothing\}$ (Ø é a identidade de $G$ ). Então existe um grupo finito $F$ e um homomorfismo $\widehat{\phi}: G \rightarrow F$ tal que $\widehat{\phi}(g)$ não é a identidade de F.

Demonstração. Seja $n$ o comprimento de $g$. Seja $X=\{0, \ldots, n\}$ e considere $F=\left\{f \in{ }^{X} X\right.$ : $f$ é bijeção $\}.(F, \circ)$ é um grupo de identidade $\operatorname{id}_{X}$. Agora, dado $a \in A$, considere $D(a)=\{i \in$ $\left.\{0, \ldots, n-1\}: g(i)=a^{-1}\right\}$ e $E(a)=\{i \in\{1, \ldots, n\}: g(i-1)=a\}$. Como $g \in G, D(a) \cap E(a)=\varnothing$. Defina $\phi(a): D(a) \cup E(a) \rightarrow X$ por: se $i \in D(a), \phi(a)(i)=i+1$; se $i \in E(a), \phi(a)(i)=i-1$. Vale que $\phi(a)$ é injetora, pois: dados $i_{1}, i_{2} \in \operatorname{dom} \phi(a)$ distintos, se $i_{1}, i_{2} \in D(a)$, então $i_{1}+1 \neq i_{2}+1$; se $i_{1}, i_{2} \in E(a)$, então $i_{1}-1 \neq i_{2}-1$; suponha $i_{1} \in D(a), i_{2} \in E(a)$ e $\phi(a)\left(i_{1}\right)=\phi(a)\left(i_{2}\right)$, ou seja $i_{1}+1=i_{2}-1$ - temos que $g\left(i_{1}\right)=a^{-1}$ e $g\left(i_{2}-1\right)=g\left(i_{1}+1\right)=a$, uma contradição.

Assim, $\phi(a)$ é injetora sobre $D(a) \cup E(a) \subset X$, e isso nos permite tomar uma extensão qualquer dela a todo o $X$ - que também denotaremos por $\phi(a)$. Portanto, temos que $\phi: A \rightarrow F$ é uma função, logo tome, pela propriedade universal dos grupos livres, $\widehat{\phi}: G \rightarrow F$ o homomorfismo que estende $\phi$.

Descreva $g=a_{0}{ }^{i_{0}} \ldots a_{n-1} i_{n-1}$, com $a_{r} \in A$ e $i_{r} \in\{1,-1\}$. Mostraremos que para cada $k \in$ $\{1, \ldots, n\}, \widehat{\phi}\left(a_{k-1} i_{k-1}\right)(k)=k-1$. Primeiro suponha que $i_{k-1}=1$; temos que $k \in E\left(a_{k-1}\right)$, logo $\phi\left(a_{k-1}\right)(k)=k-1$. Suponha agora que $i_{k-1}=-1$; temos que $k-1 \in D\left(a_{k-1}\right)$, logo $\phi\left(a_{k-1}\right)(k-1)=k$, e portanto $\widehat{\phi}\left(a_{k-1}{ }^{-1}\right)(k)=\left(\phi\left(a_{k-1}\right)\right)^{-1}(k)=k-1$.

Com isso, é fácil ver por indução que $\widehat{\phi}(g)(n)=\widehat{\phi}\left(a_{0}{ }^{i_{0}}\right) \ldots \widehat{\phi}\left(a_{n-1}{ }^{i_{n-1}}\right)(n)=0$, de forma que $\widehat{\phi}(g) \neq \mathrm{id}_{X}$.

Este próximo é o Teorema 2.24 de [1].

Teorema 3.16. Sejam A um conjunto e $G$ o grupo livre gerado por A. Então $G$ pode ser imerso em um grupo topológico compacto. Ou seja, existe um grupo topológico compacto $H$ e um homomorfismo injetor $\varphi: G \rightarrow H$.

Demonstração. Usando o teorema anterior, tome para cada $g \in G \backslash\{\varnothing\}=: G^{\prime}$ um grupo finito $F_{g}$ e um homomorfismo $\phi_{g}: G \rightarrow F_{g}$ tal que $\phi_{g}(g)$ não é a identidade de $F_{g}$. Imbua cada $F_{g}$ com a topologia discreta, e defina $H=\prod_{g \in G^{\prime}} F_{g}$, com o produto coordenada a coordenada e a topologia produto. Portanto, ao se definir $\varphi: G \rightarrow H$ por $\varphi(h)_{g}=\phi_{g}(h)$ em cada coordenada, $\varphi$ é um homomorfismo. Além disso, dado $g \in G^{\prime}$, como $\varphi(g)_{g}$ não é a identidade de $H$, temos que o núcleo de $\varphi$ é trivial e portanto $\varphi$ é injetora. Portanto, resta ver que $H$ é um grupo topológico. Para isso, basta ver que o produto é contínuo coordenada a coordenada; e dados $x, y \in H$, e $g \in G^{\prime}$, $A=\pi_{g}{ }^{-1}\left[\left\{x_{g}\right\}\right] \times \pi_{g}{ }^{-1}\left[\left\{y_{g}\right\}\right]$ é uma vizinhança de $(x, y)$ em $H \times H$ tal que $\left(\pi_{g} \circ \cdot\right)[A]=\left\{x_{g} \cdot y_{g}\right\}$. 
Enfim, o Teorema 7.35 de [1].

Teorema 3.17. Sejam $S$ semigrupo discreto, $\kappa$ cardinal infinito e $\left(a_{\lambda}\right)_{\lambda<\kappa}$ uma $\kappa$-sequência em $S$ que possui produtos finitos distintos. Considere $T=\bigcap_{\lambda<\kappa} \overline{F P\left(\left(a_{\mu}\right)_{\lambda<\mu<\kappa}\right)}$. Então $T$ é um subsemigrupo compacto de $\beta S$ tal que todo grupo maximal de $K(T)$ contém uma cópia algébrica do grupo livre sobre $2^{2^{\kappa}}$ geradores.

Demonstração. Primeiramente, para ver que $T$ é um subsemigrupo, aplicaremos a condição dada em 1.34. Sejam $\lambda<\kappa$ e $x \in F P\left(\left(a_{\mu}\right)_{\lambda<\mu<\kappa}\right)$. Tome $L \subset\{\mu: \lambda<\mu<\kappa\}$ finito tal que $x=\prod_{\mu \in L} a_{\mu}$. Defina $\gamma=\max L$. Vale que $x \cdot F P\left(\left(a_{\mu}\right)_{\gamma<\mu<\kappa}\right) \subset F P\left(\left(a_{\mu}\right)_{\lambda<\mu<\kappa}\right)$.

Pelo Teorema 1.16, todo grupo maximal em $K(T)$ é da forma $p \cdot T \cdot p$ para algum $p$ idempotente minimal de $T$. Seja então $p$ um idempotente minimal de $T$. Defina $A=\left\{a_{\mu}: \mu<\kappa\right\}$ e note que $\mathcal{U}_{\kappa}(A) \subset T$, pois dados $q \in \mathcal{U}_{\kappa}(A)$ e $\lambda<\kappa,\left\{a_{\mu}: \mu \leq \lambda\right\} \notin q$, portanto $A \backslash\left\{a_{\mu}: \mu \leq \lambda\right\}=\left\{a_{\mu}:\right.$ $\lambda<\mu<\kappa\} \in q$. Assim, podemos definir $G$ como o subgrupo de $p \cdot T \cdot p$ gerado por $p \cdot \mathcal{U}_{\kappa}(A) \cdot p$. Agora, fixe $\Sigma$ um conjunto qualquer de cardinalidade $2^{2^{\kappa}}$, e o indexe sem repetições por $\mathcal{U}_{\kappa}(A)$ : $\Sigma=\left\{\sigma_{q}: q \in \mathcal{U}_{\kappa}(A)\right\}$. Seja $F$ o grupo livre sobre $\Sigma$. Afirmamos que $G$ é isomorfo a $F$.

Pela propriedade dos grupos livres, a função definida $g: \Sigma \rightarrow p \cdot \mathcal{U}_{\kappa}(A) \cdot p$ dada por $g\left(\sigma_{q}\right)=p \cdot q \cdot p$ possui extensão a um homomorfismo $g: F \rightarrow G$. Como $G$ é o grupo gerado por $p \cdot \mathcal{U}_{\kappa}(A) \cdot p$ e $g[\Sigma]=p \cdot \mathcal{U}_{\kappa}(A) \cdot p$, segue que $g[F]=G$. Agora basta ver que $g$ é injetora. Como qualquer par de elementos de $F$ pertence a um subgrupo gerado por uma subcoleção finita de $\left\{\sigma_{q}: q \in \mathcal{U}_{\kappa}(A)\right\}$, basta ver que $g$ é injetora sobre subgrupos desse tipo.

Sejam $q_{1}, \ldots, q_{n} \in \mathcal{U}_{\kappa}(A)$ distintos e denote por $D$ o subgrupo de $F$ gerado por $\left\{\sigma_{q_{1}}, \ldots, \sigma_{q_{n}}\right\}$. Para cada $i \in\{1, \ldots, n\}$, tome $B_{i} \in q_{i}$ tal que $B_{i} \subset A$ e $B_{i} \cap B_{j}=\varnothing$ se $i \neq j$. Podemos ainda supor que $\bigcup_{i=1}^{n} B_{i}=A$ pois podemos substituir $B_{1}$ por $A \backslash \bigcup_{i=2}^{n} B_{i}$.

Definimos agora uma função $h: S \rightarrow F$. Definimos primeiro $h$ sobre $A$, determinando que $h\left(a_{\mu}\right)=$ $\sigma_{q_{i}}$ se e somente se $a_{\mu} \in B_{i}$. Após isso, dado $r \in F P\left(\left(a_{\mu}\right)_{\mu<\kappa}\right)$, como existe um único $L \in \mathcal{P}_{f}(\kappa)$ tal que $r=\prod_{\mu \in L} a_{\mu}$, podemos definir bem $h(r)=\prod_{\mu \in L} h\left(a_{\mu}\right)$. Por fim, estenda $h$ a $S$ de qualquer maneira.

Pelo teorema logo acima, $F$ pode ser imerso algebricamente em um grupo topológico compacto $\widetilde{F}$; assumiremos que $F \subset \widetilde{F}$. Seja então $\widetilde{h}: \beta S \rightarrow \widetilde{F}$ a extensão contínua de $h$. Note que $\widetilde{h}(p)$ é a identidade de $\widetilde{F}$ pois $p$ é idempotente, e que, como $h\left[B_{i}\right]=\left\{\sigma_{q_{i}}\right\}, \widetilde{h}\left(q_{i}\right)=\sigma_{q_{i}}$. Mostraremos que $\left.\widetilde{h}\right|_{T}$ é um homomorfismo. Para isso aplicaremos o Teorema 1.36, ou seja, basta ver que para cada $x \in F P\left(\left(a_{\mu}\right)_{\mu<\kappa}\right)$ existe $\lambda<\kappa$ tal que para todo $y \in F P\left(\left(a_{\mu}\right)_{\lambda<\mu<\kappa}\right), h(x \cdot y)=h(x) \cdot h(y)$. Seja então $x \in F P\left(\left(a_{\mu}\right)_{\mu<\kappa}\right)$, tome $L \in \mathcal{P}_{f}(\kappa)$ tal que $x=\prod_{\mu \in L} a_{\mu}$ e considere $\lambda=\max L$; seja $y \in F P\left(\left(a_{\mu}\right)_{\lambda<\mu<\kappa}\right)$ e tome $M \in \mathcal{P}_{f}(\kappa)$ tal que $y=\prod_{\mu \in M} a_{\mu}$ (note que $\min M>\max L$ ). Temos então: $h(x \cdot y)=h\left(\prod_{\mu \in L} a_{\mu} \cdot \prod_{\mu \in M} a_{\mu}\right)=h\left(\prod_{\mu \in L \cup M} a_{\mu}\right)=\prod_{\mu \in L \cup M} h\left(a_{\mu}\right)=\prod_{\mu \in L} h\left(a_{\mu}\right)$. 
$\prod_{\mu \in M} h\left(a_{\mu}\right)=h(x) \cdot h(y)$.

Assim $\widetilde{h}$ é um homomorfismo e portanto $\widetilde{h} \circ g$ também. Seja $i \in\{1, \ldots, n\}$. Vale que $\widetilde{h}\left(g\left(\sigma_{q_{i}}\right)\right)=$ $\widetilde{h}\left(p \cdot q_{i} \cdot p\right)=\widetilde{h}(p) \cdot \widetilde{h}\left(q_{i}\right) \cdot \widetilde{h}(p)=\widetilde{h}\left(q_{i}\right)=\sigma_{q_{i}}$. Dessa forma $\widetilde{h} \circ g$ é a identidade sobre os geradores de $D$, logo é a identidade sobre $D$, e portanto $g$ é injetora sobre $D$, como queríamos.

Note que o resultado acima vale para $T=\mathbb{H}$.

Assim se encerra a Seção 2 do artigo [9]. A Seção 3 possui apenas dois itens: um teorema e um contraexemplo a ele. Um dos itens desse teorema usa um resultado sobre $M A\left(\omega_{1}\right)$. Portanto, demonstraremos aqui uma propriedade sobre a topologia de $S^{*}$ que decorre do Axioma de Martin. Os próximos são o Lema 12.10, o Corolário 12.11 e o Corolário 12.12 do livro [1].

Lema 3.18. Seja $D$ um conjunto, $|D|=\omega$. Seja $\mathcal{A} \subset[D]^{\omega}$ com a propriedade de que para todo $\mathcal{F} \in \mathcal{P}_{f}(\mathcal{A}), D \backslash \bigcup \mathcal{F}$ é infinito. Seja $\kappa=|\mathcal{A}|$. Se vale $M A(\kappa)$, então existe $B \in[D]^{\omega}$ tal que para todo $A \in \mathcal{A}, B \cap A$ é finito.

Demonstração. Considere $Q=\left\{(H, \mathcal{F}): H \in \mathcal{P}_{f}(D)\right.$ e $\left.\mathcal{F} \in \mathcal{P}_{f}(\mathcal{A})\right\}$. Defina uma ordem em $Q$ por $\left(H^{\prime}, \mathcal{F}^{\prime}\right) \leq(H, \mathcal{F})$ e e somente se: $H \subset H^{\prime}, \mathcal{F} \subset \mathcal{F}^{\prime}$ e $\left(H^{\prime} \backslash H\right) \cap \bigcup \mathcal{F}=\varnothing$. É imediato ver que $\leq$ é reflexiva e antissimétrica. Para ver que é transitiva, sejam $H, H^{\prime}, H^{\prime \prime} \in \mathcal{P}_{f}(D)$ e $\mathcal{F}, \mathcal{F}^{\prime}, \mathcal{F}^{\prime \prime} \in \mathcal{P}_{f}(\mathcal{A})$ tais que $\left(H^{\prime \prime}, \mathcal{F}^{\prime \prime}\right) \leq\left(H^{\prime}, \mathcal{F}^{\prime}\right)$ e $\left(H^{\prime}, \mathcal{F}^{\prime}\right) \leq(H, \mathcal{F})$; a condição que não é imediata é que $\left(H^{\prime \prime} \backslash H\right) \cap \bigcup \mathcal{F}=\varnothing$. Seja $x \in H^{\prime \prime} \backslash H$. Caso $x \in H^{\prime \prime} \backslash H^{\prime}$, então $x \notin \bigcup \mathcal{F}^{\prime} \supset \bigcup \mathcal{F}$; caso $x \in H^{\prime} \backslash H$, então $x \notin \bigcup \mathcal{F}$.

Agora, se $(H, \mathcal{F})$ e $\left(H^{\prime}, \mathcal{F}^{\prime}\right)$ são incompatíveis, necessariamente $H \neq H^{\prime}$, pois do contrário $(H, \mathcal{F} \cup$ $\left.\mathcal{F}^{\prime}\right)$ é uma extensão comum de ambos. Como $\left|\mathcal{P}_{f}(D)\right|=\omega$, segue que $(Q, \leq)$ possui c.c.c.

Agora, para cada $A \in \mathcal{A}$, seja $D(A)=\{(H, \mathcal{F}) \in Q: A \in \mathcal{F}\}$. Dado $(H, \mathcal{F}) \in Q$, vale que $(H, \mathcal{F} \cup\{A\}) \in D(A)$ e $(H, \mathcal{F} \cup\{A\}) \leq(H, \mathcal{F})$, de forma que $D(A)$ é denso.

E para cada $X \in \mathcal{P}_{f}(D)$, seja $E(X)=\{(H, \mathcal{F}) \in Q: H \not \subset X\}$. Dado $(H, \mathcal{F}) \in Q$, como $D \backslash \bigcup \mathcal{F}$ é infinito, existe $y \in D \backslash \bigcup \mathcal{F}$ tal que $y \notin X$. Vale então que $(H \cup\{y\}, \mathcal{F}) \in E(X)$ e $(H \cup\{y\}, \mathcal{F}) \leq(H, \mathcal{F})$, de forma que $E(X)$ é denso.

Como $|\mathcal{A}|=\kappa \mathrm{e}\left|\mathcal{P}_{f}(D)\right|=\omega, M A(\kappa)$ nos garante que existe $G$ filtro em $(Q, \leq)$ tal que $G \cap D(A) \neq \varnothing$ para todo $A \in \mathcal{A}$ e $G \cap E(X) \neq \varnothing$ para todo $X \in \mathcal{P}_{f}(D)$.

Defina $B=\bigcup\left\{H\right.$ : existe $\mathcal{F} \in \mathcal{P}_{f}(\mathcal{A})$ tal que $\left.(H, \mathcal{F}) \in G\right\}$. Como $G \cap E(X) \neq \varnothing$ para todo $X \in \mathcal{P}_{f}(D)$, segue que $B$ é infinito (se não existiria $(H, \mathcal{F}) \in G$ tal que $H \not \subset B$ ). Afirmamos que $B \cap A$ é finito para todo $A \in \mathcal{A}$. Dado $A \in \mathcal{A}$, tome $(H, \mathcal{F}) \in G \cap D(A)$. Veremos que $B \cap A \subset H$. Seja $x \in B \cap A$. Como $x \in B$, tome $\left(H^{\prime}, \mathcal{F}^{\prime}\right) \in G$ tal que $x \in H^{\prime}$. Como $G$ é filtro, tome $\left(H^{\prime \prime}, \mathcal{F}^{\prime \prime}\right)$ extensão comum de $(H, \mathcal{F})$ e $\left(H^{\prime}, \mathcal{F}^{\prime}\right)$. Temos então que $x \in A \in \mathcal{F}, x \in H^{\prime} \subset H^{\prime \prime}$, e $\left(H^{\prime \prime} \backslash H\right) \cap \bigcup \mathcal{F}=\varnothing$; portanto, $x \in H$, como anunciamos. 
Corolário 3.19. Seja $D$ conjunto, $|D|=\omega$. Seja $\kappa<\mathfrak{c}$ e seja $\left\{F_{\alpha}: \alpha<\kappa\right\}$ uma família de fechados de $D^{*}$. Assuma $M A(\kappa)$. Se $\bigcup_{\alpha<\kappa} F_{\alpha} \neq D^{*}$, então $\operatorname{cl}_{D^{*}}\left(\bigcup_{\alpha<\kappa} F_{\alpha}\right) \neq D^{*}$.

Demonstração. Seja $x \in D^{*} \backslash \bigcup_{\alpha<\kappa} F_{\alpha}$. Para cada $\alpha<\kappa, x \notin F_{\alpha}$; como $D^{*}$ possui base de clopens, podemos tomar $D_{\alpha}$ clopen tal que $x \notin D_{\alpha}$ e $F_{\alpha} \subset D_{\alpha}$. Pelo teorema 1.26(i), cada $D_{\alpha}=A_{\alpha}{ }^{*}$ para algum $A_{\alpha} \subset D$ infinito. Temos então que $x \in D^{*} \backslash \bigcup_{\alpha<\kappa} A_{\alpha}{ }^{*}$; portanto, para cada $\alpha, D \backslash A_{\alpha} \in x$. Disso segue que a coleção $\mathcal{A}=\left\{A_{\alpha}: \alpha<\kappa\right\}$ satisfaz as hipóteses do lema anterior, e portanto existe $B \subset D$ infinito e tal que $B \cap A_{\alpha}$ é finito para cada $\alpha<\kappa$. Portanto, $B^{*} \cap \bigcup_{\alpha<\kappa} A_{\alpha}{ }^{*}=\varnothing$; como $B^{*}$ é um aberto, segue que $B^{*} \cap \operatorname{cl}_{D^{*}}\left(\bigcup_{\alpha<\kappa} A_{\alpha}{ }^{*}\right)=\varnothing$. Como $B \neq \varnothing$ e $F_{\alpha} \subset A_{\alpha}{ }^{*}$ para cada $\alpha$, segue a tese.

Corolário 3.20. Seja $D$ conjunto, $|D|=\omega$. Seja $\kappa<\mathfrak{c}$ e seja $\left\{G_{\alpha}: \alpha<\kappa\right\}$ família de abertos de $D^{*}$. Assuma $M A(\kappa)$. Se $\bigcap_{\alpha<\kappa} G_{\alpha} \neq \varnothing$, então $\operatorname{int}_{D^{*}}\left(\bigcap_{\alpha<\kappa} G_{\alpha}\right) \neq \varnothing$.

Demonstração. $\bigcap_{\alpha<\kappa} G_{\alpha} \neq \varnothing$ implica que $D^{*} \backslash \bigcap_{\alpha<\kappa} G_{\alpha} \neq D^{*} . \quad D^{*} \backslash \bigcap_{\alpha<\kappa} G_{\alpha}=\bigcup_{\alpha<\kappa} D^{*} \backslash G_{\alpha}$. Aplicando o corolário anterior, temos que $\operatorname{cl}_{D^{*}}\left(\bigcup_{\alpha<\kappa} D^{*} \backslash G_{\alpha}\right) \neq D^{*}$. $\operatorname{Mas} \operatorname{cl}_{D^{*}}\left(\bigcup_{\alpha<\kappa} D^{*} \backslash G_{\alpha}\right)=$ $\operatorname{cl}_{D^{*}}\left(D^{*} \backslash \bigcap_{\alpha<\kappa} G_{\alpha}\right)=D^{*} \backslash \operatorname{int}_{D^{*}}\left(\bigcap_{\alpha<\kappa} G_{\alpha}\right)$. Ou seja, temos que $D^{*} \backslash \operatorname{int}_{D^{*}}\left(\bigcap_{\alpha<\kappa} G_{\alpha}\right) \neq D^{*}$, e assim $\operatorname{int}_{D^{*}}\left(\bigcap_{\alpha<\kappa} G_{\alpha}\right) \neq \varnothing$.

Agora, o Teorema 3.1 de [9].

Teorema 3.21. Seja $S$ um semigrupo cancelativo à direita e fracamente cancelativo à esquerda tal que $|S|=\omega_{1}$. Então $S^{*} \backslash S^{*} S^{*}$ é denso em $S^{*}$; e se valer $M A\left(\omega_{1}\right)$, então $S^{*} S^{*}$ é nunca-denso em $S^{*}$.

Demonstração. Enumere $S=\left\{s_{\sigma}: \sigma<\omega_{1}\right\}$. Para cada $\omega<\sigma<\omega_{1}$, seja $S_{\sigma}$ o semigrupo gerado por $\left\{s_{\tau}: \tau<\sigma\right\}$. Cada $S_{\sigma}$ é enumerável e portanto, pelo Teorema 3.3, $S_{\sigma}{ }^{*} S_{\sigma}{ }^{*}$ é nunca-denso em $S_{\sigma}{ }^{*}$ (e como este é clopen, $S_{\sigma}{ }^{*} S_{\sigma}{ }^{*}$ é nunca-denso em $S^{*}$ ).

Seja $A \in[S]^{\omega}$. Mostraremos que $A^{*} \cap\left(S^{*} \backslash S^{*} S^{*}\right) \neq \varnothing$, e que se valer $M A\left(\omega_{1}\right), A^{*} \backslash \mathrm{cl} S^{*} S^{*} \neq \varnothing$. Como $A$ é enumerável, tome $\delta<\omega_{1}$ tal que $A \subset\left\{s_{\tau}: \tau<\delta\right\}$; vale que $A \subset S_{\delta}$. Além disso, $A^{*} \backslash \mathrm{cl} S_{\delta}{ }^{*} S_{\delta}{ }^{*} \neq \varnothing$, logo tome $V_{\delta} \in[A]^{\omega}$ tal que $V_{\delta}{ }^{*} \cap S_{\delta}{ }^{*} S_{\delta}{ }^{*}=\varnothing$. Agora, indutivamente seja $\delta<\sigma<\omega_{1}$ e suponha que foi escolhido $V_{\tau}$ para todo $\delta \leq \tau<\omega_{1}$ de forma que

(a) $V_{\tau} \in[A]^{\omega}$;

(b) se $\mu<\tau$, então $V_{\tau}^{*} \subset V_{\mu}{ }^{*}$; e

(c) $V_{\tau}^{*} \cap S_{\tau}^{*} S_{\tau}^{*}=\varnothing$.

Se $\sigma=\gamma+1$ para algum $\gamma$, temos que $S_{\sigma}{ }^{*} S_{\sigma}{ }^{*}$ é nunca-denso em $S^{*}$ e $V_{\gamma}{ }^{*}$ é um (já definido) aberto, de forma que $V_{\gamma}{ }^{*} \backslash \operatorname{cl} S_{\sigma}{ }^{*} S_{\sigma}{ }^{*} \neq \varnothing$ e portanto podemos tomar $V_{\sigma} \in\left[V_{\gamma}\right]^{\omega}$ de forma que 
$V_{\sigma}{ }^{*} \cap S_{\sigma}{ }^{*} S_{\sigma}{ }^{*}=\varnothing$.

Por outro lado, se $\sigma$ for limite: note que a hipótese (b) implica que $\left\{V_{\tau}: \delta \leq \tau<\sigma\right\}$ possui a $\omega$-p.i.f.. Enumere $\{\tau: \delta \leq \tau<\sigma\}=\left\{\tau_{n}: n \in \omega\right\}$. Tome $a_{0} \in V_{\tau_{0}}$ qualquer e indutivamente para $n>0$ tome $a_{n} \in \bigcap_{i=1}^{n} V_{\tau_{i}} \backslash\left\{a_{0}, \ldots, a_{n-1}\right\}$. Para cada $k \in \omega$, como $\left\{a_{n}: n \in \omega\right\} \backslash V_{\tau_{k}} \subset\left\{a_{0}, \ldots, a_{k-1}\right\}$, então $\left\{a_{n}: n \in \omega\right\}^{*} \subset V_{\tau_{k}}{ }^{*}$. Usando que $S_{\sigma}{ }^{*} S_{\sigma}{ }^{*}$ é nunca-denso em $S^{*}$, tome $V_{\sigma} \in\left[\left\{a_{n}: n \in \omega\right\}\right]^{\omega}$ tal que $V_{\sigma}{ }^{*} \cap S_{\sigma}{ }^{*} S_{\sigma}{ }^{*}=\varnothing$.

Terminada a construção, temos que $\left\{V_{\sigma}{ }^{*}: \delta \leq \sigma<\omega_{1}\right\}$ é uma família de fechados de $S^{*}$ com a p.i.f. (de fato é uma família decrescente). Logo tome $q \in \bigcap_{\delta \leq \sigma<\omega_{1}} V_{\sigma}{ }^{*}$. Vale que $q \in A^{*}$.

Afirmação: $q \notin S^{*} S^{*}$. Suponha $p, r \in S^{*}$ tais que $q=p \cdot r$. Como $A \in q$ e $|A|=\omega$, e $\mathcal{U}_{\omega_{1}}$ é ideal pelo Teorema 3.10, temos que $\|p\|=\|r\|=\omega$. Logo tome $B \in p, C \in r$ enumeráveis, e tome $\sigma<\omega_{1}$ tal que $B \cup C \subset S_{\sigma}$. Logo $p \in B^{*} \subset S_{\sigma}{ }^{*}$ e $r \in C^{*} \subset S_{\sigma}{ }^{*}$, de forma que $q \in V_{\sigma}{ }^{*} \cap S_{\sigma}{ }^{*} S_{\sigma}{ }^{*}$, uma contradição.

Por fim, assuma $M A\left(\omega_{1}\right)$. Suponha por absurdo que $A^{*} \subset \mathrm{cl} S^{*} S^{*}$. Foi observado acima que $\bigcap_{\delta \leq \sigma<\omega_{1}} V_{\sigma}{ }^{*} \neq \varnothing$. Pelo Teorema 3.20 com $D=A$, temos que $\operatorname{int}_{A^{*}}\left(\bigcap_{\delta \leq \sigma<\omega_{1}} V_{\sigma}{ }^{*}\right) \neq \varnothing$, logo tome $B \in[A]^{\omega}$ tal que $B^{*} \subset \bigcap_{\delta \leq \sigma<\omega_{1}} V_{\sigma}{ }^{*} . B^{*} \subset \operatorname{cl} S^{*} S^{*}$ e $B^{*}$ é aberto, logo $B^{*} \cap S^{*} S^{*} \neq \varnothing$. Porém, um $q \in B^{*} \cap S^{*} S^{*}$ gera a mesma contradição feita logo acima.

Note que, no exemplo dado pelo Teorema 3.7, $S$ é um semigrupo cancelativo de cardinalidade $\mathfrak{c}$ tal que $S^{*} S^{*}$ não é nunca-denso em $S^{*}$. O Teorema acima nos diz que, sob a hipótese do contínuo, $S^{*} \backslash S^{*} S^{*}$ é denso.

Quase todos os teoremas sobre a raridade de $S^{*} S^{*}$ ou a densidade de $S^{*} \backslash S^{*} S^{*}$ exigem que $S$ seja cancelativo à direita. Veremos agora que de fato não se pode enfraquecer esta hipótese de maneira significativa.

Teorema 3.22. Existe um semigrupo enumerável, cancelativo à esquerda e fracamente cancelativo $\grave{a}$ direita $S$ tal que $S^{*} S^{*}$ possui interior não-vazio em $S^{*}$.

Demonstração. Seja $L=\left\{x_{n}: n \in \mathbb{N}\right\} \cup\left\{z_{n}: n \in \mathbb{N}\right\} \cup\{y\}$ com os $x_{n}$ e $z_{n}$ todos distintos entre si e de $y$. Considere $S=\left\{a_{1} \ldots a_{t}: a_{1}, \ldots, a_{t} \in L\right.$ e se $i \in\{1, \ldots, t-1\}, a_{i}=x_{n}$ e $a_{i+1}=z_{m}$, então $n \geq m\}$. Defina então, para $u, v \in S, \operatorname{com} u=a_{1} \ldots a_{t}$ e $v=b_{1} \ldots b_{s}, u \cdot v$ como a concatenação de $u$ e $v$, exceto se $a_{t}=x_{n}$ e $b_{1}=z_{m} \operatorname{com} n<m$; nesse caso, $u \cdot v=a_{1} \ldots a_{t-1} y b_{1} \ldots b_{s}$.

Verifiquemos que é associativa: sejam $u, v, w \in S, u=a_{1} \ldots a_{t}, v=b_{1} \ldots b_{s}$ e $w=c_{1} \ldots c_{r}$.

Caso 1: $b_{1}=z_{m}$ para algum $m \in \mathbb{N}$ e $b_{s}=x_{n^{\prime}}$ para algum $n^{\prime} \in \mathbb{N}$.

Caso 1a: $a_{t} \neq x_{n}$ para todo $n<m$ e $c_{1} \neq z_{m^{\prime}}$ para todo $m^{\prime}>n^{\prime}$. Nesse caso, $u \cdot(v \cdot w)$ e $(u \cdot v) \cdot w$ são a concatenação de $u, v$ e $w$, logo são iguais. 
Caso 1b: $a_{t}=x_{n}$ para algum $n<m$ e $c_{1} \neq z_{m^{\prime}}$ para todo $m^{\prime}>n^{\prime}$. Temos

$$
\begin{aligned}
u \cdot(v \cdot w) & =\left(a_{1} \ldots a_{t}\right) \cdot\left(b_{1} \ldots b_{s} c_{1} \ldots c_{r}\right) \\
& =a_{1} \ldots a_{t-1} y b_{1} \ldots b_{s} c_{1} \ldots c_{r} \\
& =\left(a_{1} \ldots a_{t-1} y b_{1} \ldots b_{s}\right) \cdot\left(c_{1} \ldots c_{r}\right) \\
& =(u \cdot v) \cdot w .
\end{aligned}
$$

Caso 1c: $a_{t} \neq x_{n}$ para todo $n<m$ e $c_{1}=z_{m^{\prime}}$ para algum $m^{\prime}>n^{\prime}$. Temos

$$
\begin{aligned}
u \cdot(v \cdot w) & =\left(a_{1} \ldots a_{t}\right) \cdot\left(b_{1} \ldots b_{s-1} y c_{1} \ldots c_{r}\right) \\
& =a_{1} \ldots a_{t} b_{1} \ldots b_{s-1} y c_{1} \ldots c_{r} \\
& =\left(a_{1} \ldots a_{t} b_{1} \ldots b_{s}\right) \cdot\left(c_{1} \ldots c_{r}\right) \\
& =(u \cdot v) \cdot w .
\end{aligned}
$$

Caso 1d: $a_{t}=x_{n}$ para algum $n<m$ e $c_{1}=z_{m^{\prime}}$ para algum $m^{\prime}>n^{\prime}$. Temos

$$
\begin{aligned}
u \cdot(v \cdot w) & =\left(a_{1} \ldots a_{t}\right) \cdot\left(b_{1} \ldots b_{s-1} y c_{1} \ldots c_{r}\right) \\
& =a_{1} \ldots a_{t-1} y b_{1} \ldots b_{s-1} y c_{1} \ldots c_{r} \\
& =\left(a_{1} \ldots a_{t-1} y b_{1} \ldots b_{s}\right) \cdot\left(c_{1} \ldots c_{r}\right) \\
& =(u \cdot v) \cdot w .
\end{aligned}
$$

Caso 2: $b_{1} \neq z_{m}$ para todo $m \in \mathbb{N}$ e $b_{s}=x_{n^{\prime}}$ para algum $n^{\prime} \in \mathbb{N}$.

Caso 2a: $c_{1} \neq z_{m^{\prime}}$ para todo $m^{\prime}>n^{\prime}$. Nesse caso, $u \cdot(v \cdot w)$ e $(u \cdot v) \cdot w$ são a concatenação de $u, v$ e $w$, logo são iguais.

Caso 2b: $c_{1}=z_{m^{\prime}}$ para algum $m^{\prime}>n^{\prime}$. Este caso é análogo ao caso $1 \mathrm{c}$.

Caso 3: $b_{1}=z_{m}$ para algum $m \in \mathbb{N}$ e $b_{s} \neq x_{n^{\prime}}$ para todo $n^{\prime} \in \mathbb{N}$.

Caso 3a: $a_{s} \neq x_{n}$ para todo $n<m$. Nesse caso, $u \cdot(v \cdot w)$ e $(u \cdot v) \cdot w$ são a concatenação de $u, v$ e $w$, logo são iguais.

Caso 3b: $a_{s}=x_{n}$ para algum $n<m$. Este caso é análogo ao caso $1 \mathrm{~b}$.

Caso 4: $b_{1} \neq z_{m}$ para todo $m \in \mathbb{N}$ e $b_{s}=x_{n^{\prime}}$ para todo $n^{\prime} \in \mathbb{N}$. Nesse caso, $u \cdot(v \cdot w)$ e $(u \cdot v) \cdot w$ são a concatenação de $u, v$ e $w$, logo são iguais.

Agora, vejamos que $S$ é cancelativo à esquerda: sejam $u, v, w \in S, u=a_{1} \ldots a_{t}, v=b_{1} \ldots b_{s}$ e $w=c_{1} \ldots c_{r}$. Suponha que $u \cdot v=u \cdot w$. Se $a_{t} \neq x_{n}$ para todo $n \in \mathbb{N}, u \cdot v$ e $u \cdot w$ são concatenações, $\operatorname{logo} v=w$. Suponha então que $a_{t}=x_{n}$ para algum $n \in \mathbb{N}$. 
Caso a: $b_{1} \neq z_{m}$ para todo $m>n$ e $c_{1} \neq z_{m^{\prime}}$ para todo $m^{\prime}>n$. Nesse caso, $u \cdot v$ e $u \cdot w$ são concatenações, $\log 0 v=w$.

Caso b: $b_{1}=z_{m}$ para algum $m>n$ e $c_{1} \neq z_{m^{\prime}}$ para todo $m^{\prime}>n$. Temos

$u \cdot v=a_{1} \ldots a_{t-1} y b_{1} \ldots b_{s} \mathrm{e}$

$u \cdot w=a_{1} \ldots a_{t-1} a_{t} c_{1} \ldots c_{r}$.

Note que isso implica que $a_{t}=y$. Absurdo, pois $a_{t}=x_{n}$.

Caso c: $b_{1} \neq z_{m}$ para todo $m>n$ e $c_{1}=z_{m^{\prime}}$ para algum $m^{\prime}>n$. Analogamente ao caso b, temos um absurdo.

Caso d: $b_{1}=z_{m}$ para algum $m>n$ e $c_{1}=z_{m^{\prime}}$ para algum $m^{\prime}>n$. Temos

$u \cdot v=a_{1} \ldots a_{t-1} y b_{1} \ldots b_{s} \mathrm{e}$

$u \cdot w=a_{1} \ldots a_{t-1} y c_{1} \ldots c_{r}$.

Segue que $s=r$ e $b_{i}=c_{i}$ para todo $i \in\{1, \ldots, s\}$.

Agora, veremos que $S$ é fracamente cancelativo à direita. Sejam $w, t \in S$. Devemos ver que $\{s \in S: s \cdot w=t\}$ é finito. Caso seja vazio, é finito. Suponha não-vazio, e tome $u \in S$ tal que $u \cdot w=t$. O conjunto em questão pode ser descrito como $\{s \in S: s \cdot w=u \cdot w\}$. Seja $v \in S$ tal que $v \cdot w=u \cdot w$. Considerando $u=a_{1} \ldots a_{t}, v=b_{1} \ldots b_{s}$ e $w=c_{1} \ldots c_{r}$, temos: se $c_{1} \neq z_{m}$ para todo $m \in \mathbb{N}, u \cdot w$ e $v \cdot w$ são concatenações, e portanto $v=u$; ou seja, $\{s \in S: s \cdot w=u \cdot w\}=\{u\}$. Suponha agora que $c_{1}=z_{m}$ para algum $m \in \mathbb{N}$.

Caso a: $a_{t} \neq x_{n}$ para todo $n<m$. Dois subcasos: se $b_{s} \neq x_{n^{\prime}}$ para todo $n^{\prime}<m$, então $u \cdot w$ e $v \cdot w$ são concatenações, $\operatorname{logo} v=u$. Caso $b_{s}=x_{n^{\prime}}$ para algum $n^{\prime}<m$, temos

$u \cdot w=a_{1} \ldots a_{t} c_{1} \ldots c_{r} \mathrm{e}$

$v \cdot w=b_{1} \ldots b_{s-1} y c_{1} \ldots c_{r}$.

Isso implica que $s=t$ e $b_{i}=a_{i}$ para todo $i \in\{1, \ldots, t-1\}$.

Portanto, neste caso, $\{s \in S: s \cdot w=u \cdot w\} \subset\{u\} \cup\left\{a_{1} \ldots a_{t-1} x_{n^{\prime}}: n^{\prime}<m\right\}$.

Caso b: $a_{t}=x_{n}$ para algum $n<m$. Dois subcasos: se $b_{s} \neq x_{n^{\prime}}$ para todo $n^{\prime}<m$, temos

$u \cdot w=a_{1} \ldots a_{t-1} y c_{1} \ldots c_{r} \mathrm{e}$

$v \cdot w=b_{1} \ldots b_{s} c_{1} \ldots c_{r}$.

Isso implica que $s=t, b_{i}=a_{i}$ para todo $i \in\{1, \ldots, t-1\}$, e $b_{s}=y$.

Por outro lado, se $b_{s}=x_{n^{\prime}}$ para algum $n^{\prime}<m$, temos

$u \cdot w=a_{1} \ldots a_{t-1} y c_{1} \ldots c_{r} \mathrm{e}$

$v \cdot w=b_{1} \ldots b_{s-1} y c_{1} \ldots c_{r}$.

Isso implica que $s=t$ e $b_{i}=a_{i}$ para todo $i \in\{1, \ldots, t-1\}$.

Portanto, neste caso, $\{s \in S: s \cdot w=u \cdot w\} \subset\left\{u, a_{1} \ldots a_{t-1} y\right\} \cup\left\{a_{1} \ldots a_{t-1} x_{n^{\prime}}: n^{\prime}<m\right\}$. 
O resultado do teorema já implica que $S$ não é cancelativo à direita. Mas isso pode ser diretamente observado: $x_{1} z_{3}=y z_{3}=x_{2} z_{3}$ - e de fato, dado $m \in \mathbb{N}, x_{n} z_{m}=x_{n^{\prime}} z_{m}$ para todos $n, n^{\prime}<m$.

Defina agora $A=\left\{y z_{m}: m \in \mathbb{N}\right\}$. Note que $A$ é infinito. Afirmação: $A^{*} \subset S^{*} S^{*}$. Seja $p \in A^{*}$. Para cada $B \in p$, defina $C_{B}=\left\{z_{m}: y z_{m} \in B\right\} ; A \cap B \in p$, logo é infinito, e portanto $C_{B}$ também é infinito. Além disso, note que dados $B_{1}, B_{2} \in p, C_{B_{1}} \cap C_{B_{2}}=\left\{z_{m}: y z_{m} \in B_{1} \cap B_{2}\right\}=C_{B_{1} \cap B_{2}}$, de forma que $\left\{C_{B}: B \in p\right\}$ possui a $\omega$-p.i.f., o que nos permite tomar $q \in S^{*}$ tal que $\left\{C_{B}: B \in p\right\} \subset q$. Tome também $r \in\left\{x_{n}: n \in \mathbb{N}^{*}\right.$. Afirmação: $p=r \cdot q$. Basta ver que $p \subset r \cdot q$, logo seja $B \in p$. Queremos ver que $\left\{s \in S: s^{-1} B \in q\right\} \in r$, e para isso mostraremos que $\left\{x_{n}: n \in \mathbb{N}\right\} \subset\{s \in S$ : $\left.s^{-1} B \in q\right\}$. Seja $n \in \mathbb{N}$ então, e defina $D=C_{B} \cap\left\{z_{m}: m>n\right\}$. Vale que $D \in q$, e dado $z_{m} \in D$, $x_{n} z_{m}=y z_{m} \in B$, e assim $D \subset x_{n}{ }^{-1} B$, completando a demonstração. 


\subsection{Cancelabilidade à direita em $\beta S$}

Esta seção tem início na continuação do estudo do artigo "The Scarcity of Products in $\beta S \backslash S$ ", Seção 4. Como o tema é diverso das Seções 2 e 3 do artigo, e requer resultados de grupos abelianos, merece uma seção em separado nesta dissertação. Todo o material necessário sobre grupos abelianos se encontra no Apêndice A.

Dado $X$ espaço topológico, denotamos por $X_{d}$ o conjunto $X$ com a topologia discreta.

Iniciaremos por um lema que nos dá uma caracterização manuseável de cancelabilidade à direita.

Lema 3.23. Seja $S$ semigrupo infinito. Dado $p \in \beta S, p$ é cancelável à direita se e somente se para todo $A \subset S$ existe $B \subset S$ tal que $A=\left\{x \in S: x^{-1} B \in p\right\}$.

Demonstração. $(\Rightarrow)$ : p é cancelável à direita se e só se $\rho_{p}$ é injetora, e isto implica que $\rho_{p}: \beta S \rightarrow \beta S \cdot p$ é um homeomorfismo. Logo, dado $A \subset S$, temos pelo Teorema 1.26(i) que existe $B \subset S$ tal que $\bar{A} \cdot p=\bar{B} \cap \beta S \cdot p$, já que $\bar{A} \cdot p$ é um clopen de $\beta S \cdot p$. Agora, seja $x \in S$ : vale que $x \in A \Leftrightarrow x \cdot p \in \bar{A} \cdot p$, já que $p$ é cancelável à direita; e $x \cdot p \in \bar{A} \cdot p \Leftrightarrow x \cdot p \in \bar{B} \Leftrightarrow x^{-1} B \in p$. Portanto $A=\left\{x \in S: x^{-1} B \in p\right\}$.

$(\Leftarrow)$ : Suponha $s, r \in \beta S$ distintos, e tome $A \in r \backslash s$. Tome $B \subset S$ tal que $A=\left\{x \in S: x^{-1} B \in p\right\}$. Então $B \in r \cdot p \backslash s \cdot p$.

Teorema 3.24. Seja $S$ semigrupo infinito e seja T semigrupo com identidade e. Seja $p \in(S \times\{e\})^{*}$ cancelável à direita em $\beta(S \times\{e\})$. Então $p$ é cancelável à direita em $\beta(S \times T)$.

Demonstração. Usaremos o Lema acima. Seja $A \subset S \times T$. Note que a projeção $\pi_{2}: S \times T \rightarrow T$ se extende a um homomorfismo contínuo $\widetilde{\pi_{2}}: \beta(S \times T) \rightarrow \beta T$.

Para cada $t \in T$, seja $C_{t}=\{x \in S:(x, t) \in A\}$. Pelo Lema 3.23, seja $B_{t} \subset S$ tal que $C_{t} \times\{e\}=$ $\left\{(s, e) \in S \times\{e\}:(s, e)^{-1}\left(B_{t} \times\{e\}\right) \in p\right\} ;$ temos então que para todo $s \in S,(s, e) \cdot p \in \overline{B_{t} \times\{e\}} \Leftrightarrow$ $(s, e)^{-1}\left(B_{t} \times\{e\}\right) \in p \Leftrightarrow(s, e) \in C_{t} \times\{e\} \Leftrightarrow s \in C_{t} \Leftrightarrow(s, t) \in A$.

Defina $B=\bigcup_{t \in T} B_{t} \times\{t\}$. Vale que $\overline{B_{t} \times\{t\}}=\left\{x \in \bar{B}: \widetilde{\pi_{2}}(x)=t\right\}$, pois se $B_{t} \times\{t\} \in x$, por continuidade $\widetilde{\pi_{2}}(x)=t$; e se $B \backslash\left(B_{t} \times\{t\}\right) \in x$, como $t$ é um ponto isolado de $\beta T$ e $\widetilde{\pi_{2}}\left[B \backslash\left(B_{t} \times\{t\}\right)\right]=$ $T \backslash\{t\}$, segue que $\widetilde{\pi_{2}}(x) \neq t$.

Tome agora $\left(v_{i}\right)_{i \in D}$ uma rede em $S$ tal que $\left(\left(v_{i}, e\right)\right)_{i \in D}$ converge para $p$ em $\beta(S \times T)$. Seja $(s, t) \in$ $S \times T$. Mostraremos que $(s, t)^{-1} B \in p \Leftrightarrow(s, t) \in A$. Observe que para cada $i \in D,(s, t) \cdot\left(v_{i}, e\right) \in$ $B_{t} \times\{t\} \Leftrightarrow\left(s v_{i}, t\right) \in B_{t} \times\{t\} \Leftrightarrow\left(s v_{i}, e\right) \in B_{t} \times\{e\} \Leftrightarrow(s, e) \cdot\left(v_{i}, e\right) \in B_{t} \times\{e\}$. Como $\lambda_{(s, t)}$ e $\lambda_{(s, e)}$ são contínuas sobre $\beta(S \times T)$, vale que $(s, t) \cdot p=\lim _{i \in D}(s, t) \cdot\left(v_{i}, e\right)$; e $(s, e) \cdot p=\lim _{i \in D}(s, e) \cdot\left(v_{i}, e\right)$; de forma que, pelas observações anteriores, $(s, t) \cdot p \in \overline{B_{t} \times\{t\}} \Leftrightarrow(s, e) \cdot p \in \overline{B_{t} \times\{e\}}$. Por fim, note que $\widetilde{\pi_{2}}((s, t) \cdot p)=\lim _{i \in D} \pi_{2}\left((s, t) \cdot\left(v_{i}, e\right)\right)=\lim _{i \in D} \pi_{2}\left(s v_{i}, t\right)=t$. 
Temos então: $(s, t)^{-1} B \in p \Leftrightarrow(s, t) \cdot p \in \bar{B} \Leftrightarrow(s, t) \cdot p \in \overline{B_{t} \times\{t\}} \Leftrightarrow(s, e) \cdot p \in \overline{B_{t} \times\{e\}} \Leftrightarrow(s, t) \in$ $A$.

Devido a este teorema, temos que se $p \in \mathbb{N}^{*}$ é cancelável à direita em $(\beta \mathbb{N},+)$ e $T$ é um semigrupo com identidade $e$, e $q \in \beta(\mathbb{N} \times T)$ é tal que $p=\{A \subset \mathbb{N}: A \times\{e\} \in q\}$, então $q$ é cancelável em $\beta(\mathbb{N} \times\{e\})$, e, portanto, em $\beta(\mathbb{N} \times T)$. Mas por outro lado, existe $p \in \mathbb{N}^{*}$ cancelável à direita em $\beta \mathbb{N}$ que não é cancelável à direita em $\beta \mathbb{Z}$. A construção deste será dada abaixo:

Tome, pelo Teorema $1.35 q$ idempotente em $\mathbb{Z}^{*}$ tal que $q \in \bigcap_{k \geq 1} \overline{\left(F S\left(3^{t}\right)_{t \geq k}\right)}$. Defina $-q:=\{-A: A \in q\} \in \mathbb{Z}^{*}$, e considere $p=-q+q$. Como, pelo Lema 2.11, $\mathbb{N}^{*}$ é ideal à esquerda de $\beta \mathbb{Z}, p \in \mathbb{N}^{*}$. Agora veremos que $-q$ também é idempotente. Seja $A \in q$, de modo que $-A \in-q$. Queremos ver que $-A \in(-q)+(-q)$, isto é, que $\{x \in \mathbb{N}:-x+(-A) \in$ $-q\} \in-q$. Note que, dado $y \in \mathbb{N}$, se $-y+A \in q$, então $-(-y+A)=y+(-A) \in q$; de modo que $-y \in\{x \in \mathbb{N}:-x+(-A) \in-q\}$. Portanto, usando que $q$ é idempotente, temos que $-\{y \in \mathbb{N}:-y+A \in q\} \subset\{x \in \mathbb{N}:-x+(-A) \in-q\}$ e $\log 0\{x \in \mathbb{N}:-x+(-A) \in-q\} \in-q$, como queríamos.

Assim, $-q+p=-q+(-q)+q=-q+q=p$, e portanto $p$ não é cancelável à direita em $\beta \mathbb{Z}-$ se não $1+(-q)+p=1+p \Rightarrow 1+(-q)=1$, o que é absurdo.

Porém, suponha que $p$ não seja cancelável à direita em $\beta \mathbb{N}$ e tome pelo Lema 2.41 um $z \in \mathbb{N}^{*}$ tal que $p=z+p-$ ou seja, $-q+q=z+(-q)+q$. Considere agora

$$
A=\left\{\sum_{t \in F} 3^{t}-\sum_{t \in H} 3^{t}+k: k \in \mathbb{N}, F, H \in \mathcal{P}_{f}(\mathbb{N}), \min F>\max H+1, \text { e } k<3^{\min H-1}\right\}
$$

$\mathrm{e}$

$$
B=\left\{\sum_{t \in F} 3^{t}-\sum_{t \in H} 3^{t}: F, H \in \mathcal{P}_{f}(\mathbb{N}) \text { e } \min F>\max H+1\right\} .
$$

Usaremos agora o Teorema $1.31(\mathrm{~d})$ para ver que $B \in-q+q$ e que $A \in z+(-q)+q$. Seja $Q=$ $-F S\left(\left(3^{t}\right)_{t \geq 1}\right) \in-q$, e, para cada $b=-\sum_{t \in H} 3^{t} \in Q$, seja $B_{b}=\left\{\sum_{t \in F} 3^{t}: \min F>\max H+1\right\}=$ $F S\left(\left(3^{t}\right)_{t>\max H+1}\right) \in q$. Claramente, temos que $\bigcup_{b \in Q} b+B_{b} \subset B$. E para cada $k \in \mathbb{N}$, seja $A_{k}=\left\{\sum_{t \in F} 3^{t}-\sum_{t \in H} 3^{t}: F, H \in \mathcal{P}_{f}(\mathbb{N}), \min F>\max H+1\right.$, e $\left.k<3^{\min H-1}\right\}$; pode-se verificar que cada $A_{k} \in-q+q$ similarmente a como vimos que $B \in-q+q$ - usando também dessa vez que $F S\left(\left(3^{t}\right)_{t>\left\lceil\log _{3} k+1\right\rceil}\right) \in q$. Segue então que $\bigcup_{k \in \mathbb{N}} k+A_{k} \subset A$, satisfazendo a condição.

Por fim, para chegar a uma contradição, veremos que $A \cap B=\varnothing$. Para isso analisaremos a descrição de elementos de $B$ na expansão ternária. Descreva $\sum_{t \in F} 3^{t}-\sum_{t \in H} 3^{t}=\sum_{t=\min H}^{\max F} a_{t} 3^{t}$ a expansão ternária de um elemento de $B$, com $a_{t} \in\{0,1,2\}$ para todo $t$. Note que a condição $\max F>\min H+1$ implica que $a_{t} \in\{0,1\}$ para todo $t \geq \min F$; que $a_{\min F-1}=2$; que $a_{t} \in\{1,2\}$ para todo $\min H \leq t \leq \min F-1$; e que $a_{\min H}=2$. Portanto, na expansão ternária de um elemento de $B$, temos que os dígitos ternários entre o 2 de menor posição e o 2 de maior posição são 1's e 
2's; e que o dígito 2 de menor posição é o menor dígito não-nulo. Agora, um elemento de $A$ é um elemento da forma acima somado a $k<3^{\min H-1}$; seja $\sum_{t \in K} a_{t} 3^{t}$ a expansão ternária de $k$. Temos que $\max K<\min H-1$, de forma que: se $a_{\min K} \neq 2$, então pela descrição acima o elemento de $A$ não pertence a $B$ pois o menor dígito ternário não-nulo não é 2 ; e se $a_{\min K}=2$, como $a_{\min K+1}=0$, e $a_{\min H}=2$, temos um dígito 0 entre o 2 de menor posição e o 2 de maior posição, novamente implicando que o elemento de $A$ não pertence a $B$. Destarte, $A \cap B=\varnothing$.

Por outro lado, o Teorema 3.24 nos provê o seguinte:

Corolário 3.25. Seja $G$ um grupo abeliano e $H<G$ divisível. Então todo elemento cancelável à direita de $\beta H$ é cancelável à direita em $\beta G$.

Demonstração. Pelo Teorema A.10, existe $L<G$ tal que $G=H \oplus L$, de forma que o Teorema 3.24 se aplica.

Corolário 3.26. Todo elemento cancelável à direita em $\beta \mathbb{Q}_{d}$ é cancelável à direita em $\beta \mathbb{R}_{d}$, e todo elemento cancelável à direita em $\beta \mathbb{R}_{d}$ é cancelável à direita em $\beta \mathbb{C}_{d}$.

Demonstração. Use o corolário acima e o fato de que $\mathbb{Q}$ e $\mathbb{R}$ são divisíveis.

Agora, um teorema sobre a estrutura de $S^{*}$ quando $S$ é comutativo e cancelativo.

Teorema 3.27. Seja $(S,+)$ um semigrupo comutativo cancelativo e seja $G$ o seu grupo de diferenças (isto é, o menor grupo que contém $S$ ). Seja $H$ um grupo abeliano que contém $S$ (e portanto contém $G)$. Então existe $V \subset S^{*}$ satisfazendo:

(a) $V$ é aberto denso de $S^{*}$ e todo elemento de $V$ é cancelável à direita em $\beta H$.

(b) Dados $v_{1}, v_{2} \in V,\left(\beta H+v_{1}\right) \cap\left(\beta H+v_{2}\right) \neq \varnothing$ se e somente se $\left(S+v_{1}\right) \cap\left(S+v_{2}\right) \neq \varnothing$.

(c) Defina $\sim$ uma relação de equivalência sobre $S^{*}$ por: $p \sim q$ se e só se $(S+p) \cap(S+q) \neq \varnothing$. Dados $k, m \in \mathbb{N}$ e $p_{1}, \ldots, p_{m}, q_{1}, \ldots, q_{k} \in V$, se $p_{1}+\cdots+p_{m} \sim q_{1}+\cdots+q_{k}$, então $k=m e$ $p_{t} \sim q_{t}$ para cada $t \in\{1, \ldots, m\}$.

Demonstração. (a): Pelo Teorema A.3, $H$ pode ser imerso em um grupo divisível, logo assumiremos que o próprio $H$ é divisível. Seja $\mathcal{V}=\left\{A \subset[S]^{\omega}: A^{*} \cap\left(H^{*}+H^{*}\right)=\varnothing\right\}$ e defina $U=\bigcup\left\{A^{*}: A \in \mathcal{V}\right\}$. $U$ é aberto. Para ver que $U$ é denso em $S^{*}$, seja $B \in[S]^{\omega}$. Pelo Teorema $3.4, B^{*} \backslash \operatorname{cl}\left(H^{*}+H^{*}\right) \neq \varnothing$, logo como é aberto tome $A \in[S]^{\omega}$ tal que $A^{*} \subset B^{*} \backslash \operatorname{cl}\left(H^{*}+H^{*}\right)$. Vale também que $A^{*} \subset U$, mostrando que $U \cap B^{*} \neq \varnothing$.

Seja $V=\bigcup\left\{U \cap D^{*}: D\right.$ é um subgrupo divisível enumerável de $\left.H\right\}$. Vale que $V$ é aberto. Para ver que é denso, seja $B \in[S]^{\omega}$. Pelo Teorema A.18, tome $D<H$ divisível enumerável que contém $B$. Temos então, usando que $U$ é denso, que $\varnothing \neq U \cap B^{*} \subset U \cap D^{*} \subset V$. Por fim, note que cada 
$p \in V$ é tal que $\|p\|=\omega$ e $p \notin\left(H^{*}+H^{*}\right)$.

Para ver que cada $p \in V$ é cancelável, tome $D<H$ divisível enumerável tal que $p \in U \cap D^{*}$. $p \notin\left(H^{*}+H^{*}\right)$, em particular $p \notin\left(D^{*}+D^{*}\right)$, logo pelo Lema 2.41, $p$ é cancelável à direita em $\beta D$. Pelo Corolário 3.25, $p$ é cancelável à direita em $\beta H$.

(b): Sejam $v_{1}, v_{2} \in V$. Como existem $D_{1}, D_{2}<H$ divisíveis enumeráveis tais que $D_{1} \in v_{1}$ e $D_{2} \in v_{2}$, tome pelo Lema A.18 $D<H$ divisível enumerável tal que $D_{1} \cup D_{2} \subset D$; temos que $D \in v_{1} \cap v_{2}$. Pelo Teorema A.10, existe $E<H$ tal que $H=D \oplus E$. Seja $\pi_{D}$ a projeção de $H$ em $D$, e seja $\widetilde{\pi_{D}}: \beta H \rightarrow \beta D$ sua extensão contínua - que também é um homomorfismo. Note que como $\widetilde{\pi_{D}}$ é a identidade sobre $D$, segue que $\widetilde{\pi_{D}}\left(v_{1}\right)=v_{1}$ e $\widetilde{\pi_{D}}\left(v_{2}\right)=v_{2}$. Suponha então que $x+v_{1}=y+v_{2}$ para $x, y \in \beta H$. Temos que $\widetilde{\pi_{D}}(x)+v_{1}=\widetilde{\pi_{D}}(y)+v_{2}$, logo $\overline{D+v_{1}} \cap \overline{D+v_{2}} \neq \varnothing$. Como $D$ é enumerável, pelo Teorema 1.26(c), temos sem perda de generalidade que para algum $s \in D$ e algum $w \in \beta D, s+v_{1}=w+v_{2}$; definindo $z=-s+w$, temos que $v_{1}=z+v_{2}$.

Afirmação: $z \in \beta G$ (isto é, $G \in z$ ). Suponha que $D \backslash G \in z$. Como foi observado na demonstração do 3.9(b), como $G \in v_{2}, D \backslash G+G \in z+v_{2}$. Porém, dados $d \in D \backslash G$ e $g \in G, d+g \in G$ implicaria que $d \in G$, um absurdo; logo $d+g \in D \backslash G$; e portanto $D \backslash G+G \subset D \backslash G$, de forma que $D \backslash G \in z+v_{2}=v_{1} \in \beta G$, uma contradição. Logo $G \in z$.

E como $v_{1} \notin\left(H^{*}+H^{*}\right)$, temos que $z \in G$. Como $G$ é o grupo das diferenças de $S$, tome $s_{1}, s_{2} \in S$ tais que $z=s_{2}-s_{1}$. Segue que $s_{1}+v_{1}=s_{2}+v_{2}$, como queríamos.

(c):Lembre que pelo Teorema 1.38, $H$ está contido no centro de $\beta H$, e pelo $1.40, H^{*}$ é um ideal de $\beta H$.

Provaremos por indução, ou melhor, pela boa ordem: sejam $p_{1}, \ldots, p_{m}, q_{1}, \ldots, q_{k} \in V$ um contraexemplo para a afirmação tal que $k+m$ é mínimo, e tome $a, b \in S$ tais que $a+p_{1}+\cdots+p_{m}=$ $b+q_{1}+\cdots+q_{k}$. Caso $k=m=1$, não temos contradição. Caso $m=1$ e $k>1$, temos $a, b \in S$ tais que $a+p_{1}=b+q_{1}+\cdots+q_{k}$, logo temos que $p_{1}=\left(b+q_{1}\right)+\left(-a+q_{2}+\cdots+q_{k}\right) \in H^{*}+H^{*}$, uma contradição.

Portanto $k, m>2$. Como observado na demonstração do 3.9(b), $\left\{p \in S^{*}:\|p\|=\omega\right\}$ é um subsemigrupo, logo podemos tomar $A, B \in[S]^{\omega}$ tais que $A \in p_{1}+\cdots+p_{m-1}$ e $B \in q_{1}+\cdots+q_{k-1}$, de modo que $\overline{a+A+p_{m}} \cap \overline{b+B+q_{k}} \neq \varnothing$, e portanto pelo 1.26(c), temos duas opções:

(1): existem $c \in A$ e $d \in \bar{B}$ tais que $a+c+p_{m}=b+d+q_{k}$;

(2): existem $c \in A^{*}$ e $d \in B$ tais que $a+c+p_{m}=b+d+q_{k}$.

Caso valesse $(2)$, teríamos que $q_{k}=(a+c)+\left(p_{m}-b-d\right) \in H^{*}+H^{*}$, uma contradição. Logo vale $(1)$, e $p_{m}=(b+d)+\left(q_{k}-a-c\right)$. Caso $d \in B^{*}$, teríamos que $p_{m} \in H^{*}+H^{*}$, uma contradição. Logo $d \in B$, e $a+c+p_{m}=b+d+q_{k}$, mostrando que $p_{m} \sim q_{k}$. Além disso, $a+p_{1}+\cdots+p_{m-1}+b+d-a-c+q_{k}=a+p_{1} \cdots+p_{m}=b+q_{1} \cdots+q_{k}$; como $q_{k}$ é cancelável à direita pelo item (a), segue que $d+p_{1}+\cdots+p_{m-1}=c+q_{1}+\cdots+q_{k-1}$; pela minimalidade de 
$k+m$, segue que $k-1=m-1$ e $p_{t} \sim q_{t}$ para todo $t \in\{1, \ldots, m-1\}$.

O Corolário 3.25 junto com o Lema A.18 nos permitem obter mais um resultado envolvendo enumerabilidade.

Teorema 3.28. Seja $G$ um grupo infinito enumerável, subgrupo de um grupo abeliano $(H,+)$. Então todo elemento de $\beta G$ que é cancelável à direita em $\beta G$ também é cancelável à direita em $\beta H$.

Demonstração. Seja $p \in \beta G$ cancelável à direita. Em primeiro lugar, se $p \in G$, então $p \in H, \operatorname{logo} p$ é cancelável à direita em $\beta H$ (pois estamos num grupo, logo $p$ é cancelável à direita em $H$, ou seja $\left.\rho_{p}\right|_{H}$ é injetora, portanto $\rho_{p}$ é injetora sobre $\beta H$, ou seja $p$ é cancelável à direita em $\left.\beta H\right)$.

Suponha então que $p \in H^{*}$. Pelo Teorema A.3, tome $K$ grupo divisível em que $H$ está imerso. $\beta H \subset \beta K$, logo basta ver que $p$ é cancelável à direita em $\beta K$. Pelo Lema A.18, tome $D<K$ divisível enumerável tal que $G \subset D$. Pelo Corolário 3.25, basta ver que $p$ é cancelável à direita em $\beta D$. Suponha, portanto, que $p$ não é cancelável à direita em $\beta D . \quad p \in D^{*}$, logo tome pelo Lema $2.41 q \in D^{*}$ tal que $p=q+p . G \in p$, $\log \{x \in D:-x+G \in p\} \in q$. Vejamos que $\{x \in D:-x+G \in p\} \subset G$ : se $-x+G \in p$, então $(-x+G) \cap G \in p$, logo tome $g \in G$ tal que $-x+g \in G$; segue que $x \in G$. Assim, vale que $G \in q$, de forma que $p \in G^{*}+p$, e portanto $p$ não é cancelável à direita em $G$; contradição.

Para encerrar o estudo do artigo [9], o teorema a seguir diz que, por exemplo, existem $2^{2^{c}}$ ultrafiltros sobre $\mathbb{R}$ que são canceláveis à direita em $\beta \mathbb{R}_{d}$ e que convergem para um ponto fixado de $\mathbb{R}$ com respeito à topologia usual.

Teorema 3.29. Seja $S$ um semigrupo cancelativo à direita e fracamente cancelativo à esquerda com $|S|=\kappa \geq \omega$. Seja $\tau$ um topologia sobre $S$ e $x \in S$ tal que existe uma base local para $x, \mathcal{C}$, tal que $|\mathcal{C}| \leq \kappa$ e $|C|=\kappa$ para todo $C \in \mathcal{C}$. Então existe um conjunto $P \subset \beta S_{d},|P|=2^{2^{\kappa}}$ de elementos canceláveis à direita que convergem para $x$ com respeito a $\tau$.

Demonstração. Note que como $\mathcal{C}$ é base local e $|C|=\kappa$ para todo $C \in \mathcal{C}$, então $\mathcal{C}$ possui a $\kappa$-p.i.f., assim podemos aplicar o Teorema 3.14. Os itens (c) e (d), junto com o "Além disso" do enunciado, garantem o resultado desejado. 


\subsection{Cópias de $\mathbb{H}$ em $S^{*}$}

Esta seção mostra que certas condições de cancelabilidade sobre $S$ implicam na existência de cópias do semigrupo $\mathbb{H}$ em $S^{*}$. Ela foi retirada da Seção 6.3 do livro [1], e é usada na seção 2.4 desta dissertação.

Teorema 3.30. Sejam $S$ um semigrupo e $\left(x_{n}\right)_{n \geq 1}$ uma sequência em $S$ com produtos finitos distintos. Seja $T=\bigcap_{m \geq 1} \overline{F P\left(\left(x_{n}\right)_{n \geq m}\right)}$. Então T é um subsemigrupo de $\beta S$ algébrica e topologicamente isomorfo a $\mathbb{H}$.

Demonstração. Pelo Teorema 3.17, T é um subsemigrupo. Definimos $f: F P\left(x_{n}\right)_{n \geq 1} \rightarrow 2 \mathbb{N}$ por $f\left(\prod_{n \in F} x_{n}\right)=\sum_{n \in F} 2^{n}$ para cada $F \in \mathcal{P}_{f}$. Como $\left(x_{n}\right)_{n \geq 1}$ possui produtos finitos distintos, $f$ está bem-definida; além disso é sobrejetora e injetora. Portanto $\widetilde{f}: \overline{F P\left(\left(x_{n}\right)_{n \geq 1}\right)} \rightarrow \beta(2 \mathbb{N})$ é uma bijeção contínua entre compactos Hausdorff, e portanto é um homeomorfismo. Mostraremos então que $\left.\widetilde{f}\right|_{T}$ é um homomorfismo de $T$ sobre $\mathbb{H}$.

Considere $T_{m}=\operatorname{FP}\left(\left(x_{n}\right)_{n \geq m}\right)$. Observe que $f\left[T_{m}\right]=2^{m} \mathbb{N}$. Usando que $\tilde{f}$ é um homeomorfismo, temos que: $\tilde{f}[T]=\tilde{f}\left[\bigcap_{m \geq 1} \overline{T_{m}}\right]=\bigcap_{m \geq 1} \tilde{f}\left[\overline{T_{m}}\right]=\bigcap_{m \geq 1} \overline{f\left[T_{m}\right]}=\bigcap_{m \geq 1} \overline{2^{m} \mathbb{N}}=\mathbb{H}$. Para ver que $\left.\widetilde{f}\right|_{T}$ é homomorfismo, basta, pelo Teorema 1.36, ver que para cada $s \in F P\left(\left(x_{n}\right)_{n \geq 1}\right)$ existe $m \in \mathbb{N}$ tal que, para todo $t \in F P\left(\left(x_{n}\right)_{n \geq m}\right), f(s t)=f(s)+f(t)$. Seja então $s \in F P\left(\left(x_{n}\right)_{n \geq 1}\right)$ e tome $F \in \mathcal{P}_{f}(\mathbb{N})$ tal que $s=\prod_{n \in F} x_{n}$. Observe então que $m=\max F+1$ cumpre o papel desejado.

Lema 3.31. Seja $S$ um semigrupo infinito e fracamente cancelativo à esquerda. Sejam I o conjunto das identidades à direita de $S, \kappa \leq|S|$ um cardinal e $\left(s_{\lambda}\right)_{\lambda<\kappa}$ uma $\kappa$-sequência injetora em $S$. Dado $T \subset S$ de cardinalidade $\kappa$, então existe um $\kappa$-sequência injetora $\left(t_{\lambda}\right)_{\lambda<\kappa}$ em $T$ tal que

(a) dado $\mu<\kappa, t_{\mu} \notin F P\left(\left(t_{\iota}\right)_{\iota<\mu}\right)$;

(b) dados $\lambda<\mu<\kappa$ e $u, v \in I \cup\left\{s_{\iota}: \iota<\mu\right\} \cup F P\left(\left(t_{\iota}\right)_{\iota<\mu}\right), u \neq v t_{\mu}$ e $u t_{\lambda} \neq v t_{\mu}$; e

(c) $I \cap F P\left(\left(t_{\iota}\right)_{\iota<\kappa}\right)=\varnothing$.

Demonstração. Para cada $u, v \in S$, defina $A_{u, v}=\{x \in S: u=v x\}$. Como $S$ é fracamente cancelativo à esquerda, cada $A_{u, v}$ é finito. Isso implica que $I$ é finito, pois $I \subset A_{u, u}$ para qualquer $u \in S$.

$\left(t_{\lambda}\right)_{\lambda<\kappa}$ será construída indutivamente. Primeiramente tomamos $t_{0} \in T \backslash I$ qualquer. Valem (a), (b) e (c) com 0 no lugar de $\kappa$. Suponha então que definimos $\left(t_{\iota}\right)_{\iota<\nu}$ para algum $\nu<\kappa$ de forma que valham (a), (b) e (c) com $\nu$ no lugar de $\kappa$. Defina $G=I \cup\left\{s_{\iota}: \iota<\mu\right\} \cup F P\left(\left(t_{\iota}\right)_{\iota<\mu}\right)$, $H=G \cup\left\{u t_{\lambda}: u \in G\right.$ e $\left.\lambda<\nu\right\}$, e $K=\bigcup_{u, v \in H} A_{u, v}$. Note que $|G|<\kappa$, de forma que $|H|<\kappa$ e assim $|K|<\kappa$. Portanto, existe $t_{\nu} \in T \backslash(H \cup K)$. Como $t_{\nu} \notin G$, vale (a). Para ver (b), sejam 
$u, v \in G$ e $\lambda<\nu$. Como $t_{\nu} \notin A_{u, v} \cup A_{u t_{\lambda}, v}$, então $u \neq v t_{\nu}$ e $u t_{\lambda} \neq v t_{\nu}$. Por fim, dado $u \in I, t_{\nu} \neq u$ pois $t_{\nu} \notin G$ e dado $v \in F P\left(\left(t_{\iota}\right)_{\iota<\nu}, t_{\nu} \notin A_{u, v} \operatorname{logo} u \neq v t_{\nu}\right.$, de forma que vale (c).

Lema 3.32. Seja S um semigrupo cancelativo à direita e fracamente cancelativo à esquerda. Seja $T \subset S$ infinito, e seja $\kappa=|T|$. Então existe uma $\kappa$-sequência $\left(t_{\lambda}\right)_{\lambda<\kappa}$ em $T$ que possui produtos finitos distintos.

Demonstração. Seja $\left(s_{\lambda}\right)_{\lambda<\kappa}$ uma $\kappa$-sequência injetora em $S$, e tome $\left(t_{\lambda}\right)_{\lambda<\kappa} \kappa$-sequência garantida pelo Lema 3.31. Afirmamos que $\left(t_{\lambda}\right)_{\lambda<\kappa}$ possui produtos finitos distintos. Suponha que não, e sejam $F, G \in \mathcal{P}_{f}(\kappa)$ distintos tais que $\prod_{\lambda \in F} t_{\lambda}=\prod_{\lambda \in G} t_{\lambda}$, com $|F \cup G|$ mínimo entre tais contraexemplos. Sem perda de generalidade, $\max F \leq \max G=\mu$. Suponha que $\max F<\max G$ : isso contradiz o item (a) do Lema 3.31 caso $G=\{\mu\}$, e o item (b) caso contrário. Logo max $F=\mu$. Caso $|F|,|G|>$ 1, poderíamos cancelar $t_{\mu}$ à direita e obter um contraexemplo que contradiria a minimalidade de $|F \cup G|$. Logo, sem perda de generalidade, $|F|=1$. Note que $|G|=1$ implicaria $F=G$. Logo $|G|>1$, e podemos considerar $x=\prod_{\lambda \in G \backslash\{\mu\}} t_{\lambda}$. Temos então que $t_{\mu}=\prod_{\lambda \in F} t_{\lambda}=\prod_{\lambda \in G} t_{\lambda}=x t_{\mu}$; $\operatorname{logo}$, para todo $s \in S, s t_{\mu}=s x t_{\mu}$; cancelando à direita obtemos $s=s x$, para todo $s \in S$; ou seja, $x \in I$. Isso contradiz o item (c) do Lema 3.31.

Teorema 3.33. Seja $S$ um semigrupo infinito, cancelativo à direita e fracamente cancelativo à esquerda. Seja $A \subset S^{*}$ um $G_{\delta}$ que possui um idempotente. Então A contém uma cópia algébrica e topológica de $\mathbb{H}$.

Demonstração. Seja $p \in A$ idempotente. Seja $\left(A_{m}\right)_{m \geq 1}$ é uma sequência decrescente de subconjuntos de $S$ tal que $\bigcap_{m \geq 1} \overline{A_{m}} \subset A$. Construiremos uma sequência $\left(x_{n}\right)_{n \geq 1}$ em $S$ tal que, para cada $m \geq 1, F P\left(\left(x_{n}\right)_{n \geq m}\right) \subset A_{m}^{\star}(p)$. Inicie tomando $x_{1} \in A_{1}^{\star}(p)$ qualquer. Agora, suponha $x_{1}, \ldots, x_{k}$ escolhidos de forma que, para cada $i \in\{1, \ldots, k\}, F P\left(\left(x_{n}\right)_{i \leq n \leq k}\right) \subset A_{i}{ }^{\star}(p)$. Temos que $A_{k+1}^{\star}(p) \in p$ pois $p$ é idempotente, e pelo Teorema 1.33, para cada $i \in\{1, \ldots, k\}$ e para cada $y \in$ $F P\left(\left(x_{n}\right)_{i \leq n \leq k}\right), y^{-1}\left(A_{i}^{\star}(p)\right) \in p$. Desta forma, podemos tomar $x_{k+1} \in A_{k+1}{ }^{\star}(p) \cap \bigcap\left\{y^{-1}\left(A_{i}^{\star}(p)\right)\right.$ : $i \in\{1, \ldots, k\}$ e $\left.y \in F P\left(\left(x_{n}\right)_{i \leq n \leq k}\right)\right\} \backslash\left\{x_{1}, \ldots, x_{k}\right\}$. Dessa forma, para cada $i \in\{1, \ldots, k+1\}$, $F P\left(\left(x_{n}\right)_{i \leq n \leq k}\right) \subset A_{i}^{\star}(p)$.

Agora, aplicando o Lema 3.32, com $T=\left\{x_{n}: n \in \mathbb{N}\right\}$, obtemos uma sequência $\left(t_{n}\right)_{n \geq 1}$ em $T$ que possui produtos finitos distintos; $\left(t_{n}\right)_{n \geq 1}$ é em particular injetora, logo podemos refiná-la a uma subsequência $\left(s_{n}\right)_{n \geq 1}$ que também é uma subsequência de $\left(x_{n}\right)_{n \geq 1}$. Assim, sendo subsequência de $\left(t_{n}\right)_{n \geq 1},\left(s_{n}\right)_{n \geq 1}$ possui a unicidade dos produtos finitos; e sendo subsequência de $\left(x_{n}\right)_{n \geq 1}$, vale que para cada $m \geq 1, F P\left(\left(s_{n}\right)_{n \geq m}\right) \subset F P\left(\left(x_{n}\right)_{n \geq m}\right) \subset A_{m}^{\star}(p) \subset A_{m}$. Dessa forma, $\bigcap_{m \geq 1} \overline{F P\left(\left(s_{n}\right)_{n \geq m}\right)} \subset \bigcap_{m \geq 1} \overline{A_{m}} \subset A$. E o Teorema 3.30 nos diz que $\bigcap_{m \geq 1} \overline{F P\left(\left(s_{n}\right)_{n \geq m}\right)}$ é uma cópia algébrica e topológica de $\mathbb{H}$, pois $\left(s_{n}\right)_{n \geq 1}$ possui a unicidade dos produtos finitos. 


\subsection{O Teorema de Zelenyuk}

Esta seção apresenta todos os passos necessários para se obter o Teorema de Zelenyuk: não existem subgrupos finitos não-triviais em $\beta \mathbb{N}$. Aqui, por "subgrupo trivial" queremos dizer grupos unitários formados por um idempotente. Este é um resultado surpreendente, e vai ao encontro da tendência a ser cada vez mais fácil encontrar subgrupos quanto maior é sua cardinalidade exigida: o Teorema 3.17 junto com o 3.32 diz que existem muitas cópias do grupo livre sobre $2^{2^{\kappa}}$ geradores em $S^{*}$ para $S$ cancelativo à direita e fracamente cancelativo à esquerda. Note também que para qualquer $p \in \mathbb{N}^{*}$ idempotente, $\mathbb{Z}+p$ é uma cópia algébrica de $\mathbb{Z}$. O Teorema de Zelenyuk, um resultado de Yevhen Zelenyuk de 1996, pôs fim às indagações sobre subgrupos finitos.

O artigo original ([12]) é em russo, e por isso apresentamos aqui a prova como é apresentada na seção 7.1 do livro [1]. Esta seção está no capítulo sobre $\beta S$, e não sobre $\beta \mathbb{N}$, pois o enunciado do Teorema de Zelenyuk é abrangente: se $G$ é um grupo enumerável sem subgrupos finitos não-triviais, então $G^{*}$ não possui subgrupos finitos não-triviais.

Definição 3.34. Seja $G$ um grupo. Uma topologia $\tau$ em $G$ é dita ser invariante à esquerda se para todo $U \in \tau$ e todo $a \in G, a \cdot U \in \tau$.

\section{Observações:}

Uma topologia em $G$ é invariante à esquerda se e somente se $\lambda_{a}$ é um homeomorfismo para todo $a \in G$, pois: Se $\lambda_{a}$ é homeomorfismo para todo $a \in G$, então dado $U$ aberto, $a \cdot U=\lambda_{a}[U]$ é aberto. E se $a \cdot U=\lambda_{a}[U]$ é aberto para todo $a \in G$ e todo $U$ aberto, então temos que $\lambda_{a}$ é uma função aberta; e como $\left(\lambda_{a}\right)^{-1}=\lambda_{a^{-1}}$, para todo $U$ aberto vale que $\lambda_{a}{ }^{-1}[U]$ é aberto, $\operatorname{logo} \lambda_{a}$ é contínua; como $\lambda_{a}$ é bijetora, ela é homeomorfismo.

E $\lambda_{a}$ é homeomorfismo para cada $a \in G$ se e somente se $(G, \cdot, \tau)$ é um grupo topológico à esquerda, pois: Se $\lambda_{a}$ é homeomorfismo para todo $a \in G$, em particular $\lambda_{a}$ é contínua para cada $a \in G, \operatorname{logo} G$ é topológico à esquerda. Por outro lado se $G$ é topológico à esquerda, então $\lambda_{a}$ é contínua para todo $a \in G$; em particular, dado $a \in G, \lambda_{a}$ e $\lambda_{a^{-1}}=\left(\lambda_{a}\right)^{-1}$ são contínuas, $\operatorname{logo} \lambda_{a}$ é um homeomorfismo.

Definição 3.35. (a) $F$ denotará o semigrupo livre sobre as letras 0 e 1, com identidade $\varnothing$.

(b) Dados $m \in \omega$ e $i \in m+1, s_{i}^{m}$ denotará o elemento de $F$ formado por $i$ 0's seguido de $m-i$ 1's. Ou seja, formalmente, $\operatorname{dom}\left(s_{i}^{m}\right)=\{1, \ldots, m\}, s_{i}^{m}(k)=0$ caso $1 \leq k \leq i$ e $s_{i}^{m}(k)=1$ caso $i<k \leq m$. Também definimos $u_{m}=s_{m}^{m}$ (de forma que $u_{0}=s_{0}^{0}=\varnothing$ ).

(c) Dado $s \in F, l(s)=|\operatorname{dom}(s)|$ denotará o comprimento de $s$, e $\operatorname{supp}(s)=\{i \in\{1, \ldots, l(s)\}$ : $s(i)=1\}$.

(d) Dados $s, t \in F$, diremos que $s<<t$ se $\max \operatorname{supp}(s)+1<\min \operatorname{supp}(t)$. 
(e) Dados $s, t \in F$, definimos $s+t \in F$ por $l(s+t)=\max \{l(s), l(t)\}$ e $(s+t)(i)=1$ se e só se $s(i)=1$ ou $t(i)=1$.

O próximo lema mostra que tais $s_{i}^{m}$ são os blocos básicos que compõem os elementos de $F$.

Lema 3.36. (a) A operação + definida em F acima é associativa e comutativa.

(b) Dados $k \in \mathbb{N}$, e $0 \leq i_{0}<m_{0}<i_{1}<m_{1}<\cdots<i_{k} \leq m_{k}$, considere $t=s_{i_{0}}^{m_{0}}+\cdots+s_{i_{k}}^{m_{k}}$. Então dado $j \in\{0, \ldots, k\}$ e dado $l$ tal que $0 \leq l \leq m_{j}$, vale que $t(l)=s_{i_{0}}^{m_{0}}+\cdots+s_{i_{j}}^{m_{j}}(l)$.

(b') Dados $k \in \mathbb{N}$, e $0 \leq i_{0}<m_{0}<i_{1}<m_{1}<\cdots<i_{k} \leq m_{k}$, considere $t=s_{i_{0}}^{m_{0}}+\cdots+s_{i_{k}}^{m_{k}}$. Então dado $j \in\{1, \ldots, k\}$ e dado $l$ tal que $m_{j-1}<l \leq m_{k}$, vale que $t(l)=s_{i_{j}}^{m_{j}}+\cdots+s_{i_{k}}^{m_{k}}(l)$.

(c) Cada $t \in F$ possui um representação única na forma $t=s_{i_{0}}^{m_{0}}+\cdots+s_{i_{k}}^{m_{k}} \operatorname{com} 0 \leq i_{0}<m_{0}<$ $i_{1}<m_{1}<\cdots<i_{k} \leq m_{k}$ (caso $k=0$, a exigência é $\left.0 \leq i_{0} \leq m_{0}\right)$.

Demonstração. (a): Isso segue da associatividade e comutatividade do operador max e do operador lógico "ou".

(b): Pela definição, $t(l)=1$ se e somente se existe $r \in\{0, \ldots, k\}$ tal que $s_{i_{r}}^{m_{r}}(l)=1$. Como $l \leq m_{j}<i_{j+1}<\cdots<i_{k}$, então $s_{i_{r}}^{m_{r}}(l)=0$ para $r \in\{j+1, \ldots, k\}$. Logo, resta que $t(l)=1$ se e somente se $s_{i_{r}}^{m_{r}}(l)=1$ para algum $0 \leq r \leq j$, ou seja, $t(l)=1$ se e somente se $s_{i_{0}}^{m_{0}}+\cdots+s_{i_{j}}^{m_{j}}(l)=1$. Como consequência, $t(l)=0$ se somente se $s_{i_{0}}^{m_{0}}+\cdots+s_{i_{j}}^{m_{j}}(l)=1$; portanto, $t(l)=s_{i_{0}}^{m_{0}}+\cdots+s_{i_{j}}^{m_{j}}(l)$. (b'): Pela definição, $t(l)=1$ se e somente se existe $r \in\{0, \ldots, k\}$ tal que $s_{i_{r}}^{m_{r}}(l)=1$. Como $i_{0}<$ $m_{0}<i_{1}<m_{1}<\cdots<i_{j-1}<m_{j-1}<l$, então $s_{i_{r}}^{m_{r}}$ não está definido em $l$ para $r \in\{0, \ldots, j-1\}$. Logo, resta que $t(l)=1$ se e somente se $s_{i_{r}}^{m_{r}}(l)=1$ para algum $j \leq r \leq k$, ou seja, $t(l)=1$ se e somente se $s_{i_{j}}^{m_{j}}+\cdots+s_{i_{k}}^{m_{k}}(l)=1$. Como consequência, $t(l)=0$ se somente se $s_{i_{j}}^{m_{j}}+\cdots+s_{i_{k}}^{m_{k}}(l)=1$; portanto, $t(l)=s_{i_{j}}^{m_{j}}+\cdots+s_{i_{k}}^{m_{k}}(l)$.

(c): Provaremos a existência por indução sobre $l(t)$. (Usaremos com frequência os itens (b) e (b').) Caso $l(t)=0, t=\varnothing, \operatorname{logo} t=s_{0}^{0}$, e caso $l(t)=1, t=0$ ou $t=1, \operatorname{logo} t=s_{1}^{1}$ ou $t=s_{0}^{1}$. Agora suponha $l(t)=n+1$ e tome $s_{i_{0}}^{m_{0}}+\cdots+s_{i_{k}}^{m_{k}}$ a representação de $\left.t\right|_{\{1, \ldots, n\}}$, com $0 \leq i_{0}<m_{0}<i_{1}<m_{1}<\cdots<i_{k} \leq m_{k}$. Observe que $n=m_{k}\left(\right.$ pois $\left.l\left(s_{i_{0}}^{m_{0}}+\cdots+s_{i_{k}}^{m_{k}}\right)=m_{k}\right)$.

Caso $t(n)=0:$ temos que $s_{i_{k}}^{m_{k}}\left(m_{k}\right)=0, \operatorname{logo} i_{k}=m_{k}$.

Subcaso $t(n+1)=0$ : basta verificar que $t=s_{i_{0}}^{m_{0}}+\cdots+s_{i_{k-1}}^{m_{k-1}}+s_{m_{k}+1}^{m_{k}+1}$. Claramente se $0 \leq$ $l \leq m_{k-1}, t(l)=s_{i_{0}}^{m_{0}}+\cdots+s_{i_{k-1}}^{m_{k-1}}(l)=s_{i_{0}}^{m_{0}}+\cdots+s_{i_{k-1}}^{m_{k-1}}+s_{m_{k}+1}^{m_{k}+1}(l)$. Se $m_{k-1}<l \leq m_{k}=$ $n$, então $t(l)=s_{i_{0}}^{m_{0}}+\cdots+s_{i_{k}}^{m_{k}}(l)=s_{m_{k}}^{m_{k}}(l)=0=s_{m_{k}+1}^{m_{k}+1}(l)=s_{i_{0}}^{m_{0}}+\cdots+s_{i_{k-1}}^{m_{k-1}}+s_{m_{k}+1}^{m_{k}+1}(l)$. Por fim, $t\left(m_{k}+1\right)=0=s_{m_{k}+1}^{m_{k}+1}\left(m_{k}+1\right)=s_{i_{0}}^{m_{0}}+\cdots+s_{i_{k-1}}^{m_{k-1}}+s_{m_{k}+1}^{m_{k}+1}\left(m_{k}+1\right)$. 
Subcaso $t(n+1)=1$ : basta verificar que $t=s_{i_{0}}^{m_{0}}+\cdots+s_{i_{k-1}}^{m_{k-1}}+s_{m_{k}}^{m_{k}+1}$. Claramente se $0 \leq$ $l \leq m_{k-1}, t(l)=s_{i_{0}}^{m_{0}}+\cdots+s_{i_{k-1}}^{m_{k-1}}(l)=s_{i_{0}}^{m_{0}}+\cdots+s_{i_{k-1}}^{m_{k-1}}+s_{m_{k}}^{m_{k}+1}(l)$. Se $m_{k-1}<l \leq m_{k}=$ $n$, então $t(l)=s_{i_{0}}^{m_{0}}+\cdots+s_{i_{k}}^{m_{k}}(l)=s_{m_{k}}^{m_{k}}(l)=0=s_{m_{k}}^{m_{k}+1}(l)=s_{i_{0}}^{m_{0}}+\cdots+s_{i_{k-1}}^{m_{k-1}}+s_{m_{k}}^{m_{k}+1}(l)$. Por fim, $t\left(m_{k}+1\right)=1=s_{m_{k}}^{m_{k}+1}\left(m_{k}+1\right)=s_{i_{0}}^{m_{0}}+\cdots+s_{i_{k-1}}^{m_{k-1}}+s_{m_{k}}^{m_{k}+1}\left(m_{k}+1\right)$.

Caso $t(n)=1:$ Temos que $s_{i_{k}}^{m_{k}}\left(m_{k}\right)=1, \log i_{k}<m_{k}$.

Subcaso $t(n+1)=1$ : basta verificar que $t=s_{i_{0}}^{m_{0}}+\cdots+s_{i_{k-1}}^{m_{k-1}}+s_{i_{k}}^{m_{k}+1}$. Claramente se $0 \leq$ $l \leq m_{k-1}, t(l)=s_{i_{0}}^{m_{0}}+\cdots+s_{i_{k-1}}^{m_{k-1}}(l)=s_{i_{0}}^{m_{0}}+\cdots+s_{i_{k-1}}^{m_{k-1}}+s_{i_{k}}^{m_{k}+1}(l)$. Se $m_{k-1}<l \leq m_{k}=$ $n$, então $t(l)=s_{i_{0}}^{m_{0}}+\cdots+s_{i_{k}}^{m_{k}}(l)=s_{i_{k}}^{m_{k}}(l)=s_{i_{k}}^{m_{k}+1}(l)=s_{i_{0}}^{m_{0}}+\cdots+s_{i_{k-1}}^{m_{k-1}}+s_{i_{k}}^{m_{k}+1}(l)$. Por fim, $t\left(m_{k}+1\right)=1=s_{i_{k}}^{m_{k}+1}\left(m_{k}+1\right)=s_{i_{0}}^{m_{0}}+\cdots+s_{i_{k-1}}^{m_{k-1}}+s_{i_{k}}^{m_{k}+1}\left(m_{k}+1\right)$.

Subcaso $t(n+1)=0$ : basta verificar que $t=s_{i_{0}}^{m_{0}}+\cdots+s_{i_{k}}^{m_{k}}+s_{m_{k}+1}^{m_{k}+1}\left(\operatorname{como} i_{k}<m_{k}<\right.$ $m_{k}+1$, esta é uma representação na forma desejada). Claramente se $0 \leq l \leq m_{k}=n$, $t(l)=s_{i_{0}}^{m_{0}}+\cdots+s_{i_{k}}^{m_{k}}(l)=s_{i_{0}}^{m_{0}}+\cdots+s_{i_{k}}^{m_{k}}+s_{m_{k}+1}^{m_{k}+1}(l) . \mathrm{E} t\left(m_{k}+1\right)=0=s_{m_{k}+1}^{m_{k}+1}\left(m_{k}+1\right)=$ $s_{i_{0}}^{m_{0}}+\cdots+s_{i_{k}}^{m_{k}}+s_{m_{k}+1}^{m_{k}+1}\left(m_{k}+1\right)$.

Assim, provamos a existência de tal representação. Vejamos a unicidade, usando o princípio da boa ordem: suponha por absurdo que temos um contraexemplo, ou seja, $k, l \in \omega, 0 \leq i_{0}<$ $m_{0}<i_{1}<m_{1}<\cdots<i_{k} \leq m_{k}$ e $0 \leq j_{0}<n_{0}<j_{1}<n_{1}<\cdots<j_{l} \leq n_{l}$ de forma que $s_{i_{0}}^{m_{0}}+\cdots+s_{i_{k}}^{m_{k}}=s_{j_{0}}^{n_{0}}+\cdots+s_{j_{l}}^{n_{l}}$, mas $\left(i_{0}, m_{0}, i_{1}, m_{1}, \ldots, i_{k}, m_{k}\right) \neq\left(j_{0}, n_{0}, j_{1}, n_{1}, \ldots, j_{l}, n_{l}\right)$, com

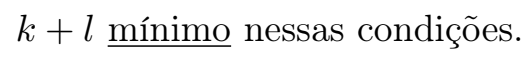

Em primeiro lugar, observe que $m_{k}=n_{l}$, pois esses são os respectivos comprimentos das palavras. Suponha que $i_{k}<j_{l}$ : temos que $1=s_{i_{0}}^{m_{0}}+\cdots+s_{i_{k}}^{m_{k}}\left(i_{k}+1\right)=s_{j_{0}}^{n_{0}}+\cdots+s_{j_{l}}^{m_{k}}\left(i_{k}+1\right)=0$, uma contradição. Similarmente, não se pode ter que $j_{l}<i_{k}$, logo $i_{k}=j_{l}$. Ou seja, temos que $s_{i_{0}}^{m_{0}}+\cdots+s_{i_{k-1}}^{m_{k-1}}+s_{i_{k}}^{m_{k}}=s_{j_{0}}^{n_{0}}+\cdots+s_{j_{l-1}}^{n_{l-1}}+s_{i_{k}}^{m_{k}}$.

Caso $k=l=0, i_{0}=j_{0}$ e $m_{0}=n_{0}$, ou seja $\left(i_{0}, m_{0}\right)=\left(j_{0}, n_{0}\right)$, e não temos um contraexemplo.

Logo suponha $k=0$ e $l>0$. Temos que $j_{0}<n_{0}<j_{l}=i_{0}$, portanto $0=s_{i_{0}}^{m_{0}}\left(j_{0}+1\right)=$ $s_{j_{0}}^{n_{0}}+\cdots+s_{j_{l}}^{n_{l}}\left(j_{0}+1\right)=s_{j_{0}}^{n_{0}}\left(j_{0}+1\right)=1$, uma contradição.

Portanto, suponha que $k, l>0$. Temos que dado $0 \leq l \leq m_{k-1}$, vale que $s_{i_{0}}^{m_{0}}+\cdots+s_{i_{k-1}}^{m_{k-1}}(l)=$ $s_{i_{0}}^{m_{0}}+\cdots+s_{i_{k-1}}^{m_{k-1}}+s_{i_{k}}^{m_{k}}(l)=s_{j_{0}}^{n_{0}}+\cdots+s_{j_{l-1}}^{n_{l-1}}+s_{i_{k}}^{m_{k}}(l)=s_{j_{0}}^{n_{0}}+\cdots+s_{j_{l-1}}^{n_{l-1}}(l)$. Dessa forma, $s_{i_{0}}^{m_{0}}+\cdots+s_{i_{k-1}}^{m_{k-1}}=s_{j_{0}}^{n_{0}}+\cdots+s_{j_{l-1}}^{n_{l-1}}$. Como $k-1+l-1<k+l$, a minimalidade de $k+l$ garante que $\left(i_{0}, m_{0}, i_{1}, m_{1}, \ldots, i_{k-1}, m_{k-1}\right)=\left(j_{0}, n_{0}, j_{1}, n_{1}, \ldots, j_{l-1}, n_{l-1}\right)$. Porém, já provamos que $i_{k}=j_{l}$ e $m_{k}-n_{l}$, ou seja, $\left(i_{0}, m_{0}, i_{1}, m_{1}, \ldots, i_{k}, m_{k}\right)=\left(j_{0}, n_{0}, j_{1}, n_{1}, \ldots, j_{l}, n_{l}\right)$, e assim não temos um contraexemplo. Contradição.

Definição 3.37. (a) Dado $t \in F$, sua representação única na forma $t=s_{i_{0}}^{m_{0}}+\cdots+s_{i_{k}}^{m_{k}}$, com $k \in \omega$ e $0 \leq i_{0}<m_{0}<i_{1}<m_{1}<\cdots<i_{k} \leq m_{k}$ (caso $k=0$, a exigência é $0 \leq i_{0} \leq m_{0}$ ), será chamada de representação canônica de $t$. 
(b) Dado $t \in F$, se $t=s_{i}^{m}$ para algum $i, m \in \omega$, definimos $t^{\prime}=\varnothing$ e $t^{*}=t$. Caso contrário, com $t=s_{i_{0}}^{m_{0}}+\cdots+s_{i_{k+1}}^{m_{k+1}}$, definimos $t^{\prime}=s_{i_{0}}^{m_{0}}+\cdots+s_{i_{k}}^{m_{k}}$ e $t^{*}=s_{i_{k+1}}^{m_{k+1}}$.

(c) Definimos $c: F \rightarrow \omega$ por $c(t)=|\operatorname{supp}(t)|$.

(d) Para cada $p \in \mathbb{N}$, definimos $g_{p}: F \rightarrow \mathbb{Z}_{p}$ por $g_{p}(t)=[c(t)]_{p}$ (ou seja, $g_{p}(t)$ é a $p$-classe de $c(t)$ ).

Lembramos que a operação no semigrupo $F$ é a concatenação, denotada por $\frown$.

Vale notar que dados $s, t \in F$, se $\operatorname{supp}(s) \cap \operatorname{supp}(t)=\varnothing$, então $c(s+t)=c(s)+c(t)$ e logo, para cada $p \in \mathbb{N}, g_{p}(s+t)=g_{p}(s)+g_{p}(t)$. Em particular, isso vale caso $s<<t$.

Agora, nos será útil determinar a representação canônica de $s+t$ quando $s<<t$. Sejam então $s=s_{i_{0}}^{m_{0}}+\cdots+s_{i_{k}}^{m_{k}}$ e $t=s_{j_{0}}^{n_{0}}+\cdots+s_{j_{l}}^{n_{l}}$ as representações canônicas de $s$ e $t$. Primeiramente, note que $\min \operatorname{supp}(t)=j_{0}+1$. Caso $i_{k}<m_{k}$, temos que $\max \operatorname{supp}(s)=m_{k}, \operatorname{logo} m_{k}+1<j_{0}+1$, assim $m_{k}<j_{0}$, e portanto $s_{i_{0}}^{m_{0}}+\cdots+s_{i_{k}}^{m_{k}}+s_{j_{0}}^{n_{0}}+\cdots+s_{j_{l}}^{n_{l}}$ é a representação canônica de $s+t$. Porém, caso $i_{k}=m_{k}$, então temos que $\max \operatorname{supp}(s)=m_{k-1}$, logo $m_{k-1}<j_{0}$. Se $m_{k}<n_{l}$, note que $s_{m_{k}}^{m_{k}}+t=t$, de forma que $s+t=s_{i_{0}}^{m_{0}}+\cdots+s_{i_{k-1}}^{m_{k-1}}+s_{j_{0}}^{n_{0}}+\cdots+s_{j_{l}}^{n_{l}}$ é a representação canônica de $s+t$. E se $m_{k}>n_{l}$, então $s_{i_{0}}^{m_{0}}+\cdots+s_{i_{k-1}}^{m_{k-1}}+s_{j_{0}}^{n_{0}}+\cdots+s_{j_{l}}^{n_{l}}+s_{m_{k}}^{m_{k}}$ é a representação canônica de $s+t$.

Seja $t \in F, l(t)=n$, e defina $v=t \frown 1$ e $w=t \frown 0$. Usando $s_{i_{0}}^{m_{0}}+\cdots+s_{i_{k}}^{m_{k}}$ a representação canônica de $t$, e o lema acima, temos que, caso $t(n)=0, w=s_{i_{0}}^{m_{0}}+\cdots+s_{i_{k-1}}^{m_{k-1}}+s_{m_{k}+1}^{m_{k}+1}$, de modo que $t=\left(w^{\prime}\right) \frown\left(u_{m_{k}+1-m_{k-1}}\right)$ e $w^{*}=u_{m_{k}+1}$; e caso $t(n)=1, w=s_{i_{0}}^{m_{0}}+\cdots+s_{i_{k}}^{m_{k}}+s_{m_{k}+1}^{m_{k}+1}$, de modo que $t=w^{\prime}$ e $w^{*}=u_{m_{k}+1}$. E em ambos os casos, $v=s_{i_{0}}^{m_{0}}+\cdots+s_{i_{k}}^{m_{k}+1}$, de modo que $v^{\prime}=t^{\prime}$ e $v^{*}=t^{*} \frown 1$.

Em suma: se $t \in F$ e $l(t)=n, v^{\prime}=t^{\prime}, v^{*}=t^{* \frown 1}, w^{*}=u_{n+1}$ e existe $r \in \omega$ tal que $t=\left(w^{\prime}\right) \frown\left(u_{r}\right)$.

Lema 3.38. Sejam $G$ um grupo com identidade e, e $X \subset G$ enumerável tal que e $\in X$. Suponha que $G$ possui uma topologia invariante à esquerda, Hausdorff, e zero dimensional, tal que X não possui pontos isolados na topologia de subespaço, e que, para todo $a \in X, a \cdot X \cap X$ é uma vizinhança de a em $X$. Além disso, suponha que $p \in \mathbb{N}$ e que existe uma função $h: X \rightarrow \mathbb{Z}_{p}$ de forma que, para cada $a \in X$, existe $V(a)$ uma vizinhança de e em $X$ tal que $a \cdot V(a) \subset X$ e $h(a \cdot b)=h(a)+h(b)$ para todo $b \in V(a)$; que $h[Y]=\mathbb{Z}_{p}$ para todo $Y$ aberto de $X$; e que $V(e)=X$.

Então é possivel definir $x(t) \in X$ e $X(t) \subset X$ para todo $t \in F$ de forma que $x(\varnothing)=e, X(\varnothing)=X$, e as seguintes condições estejam satisfeitas:

(1) $X(t)$ é um clopen de $X$.

(2) $x(t) \in X(t)$.

(3) $X\left(t^{\frown} 0\right) \cup X\left(t^{\frown} 1\right)=X(t)$ e $X\left(t^{\frown} 0\right) \cap X\left(t^{\frown} 1\right)=\varnothing$. 
(4) $x\left(t^{\frown 0)}=x(t)\right.$.

(5) $x(t)=x\left(t^{\prime}\right) \cdot x\left(t^{*}\right)$.

(6) $X(t)=x\left(t^{\prime}\right) \cdot X\left(t^{*}\right)$.

(7) $X\left(t^{*}\right) \subset V\left(x\left(t^{\prime}\right)\right)$

(8) $h(x(t))=g_{p}(t)$.

(9) Dados $s, t \in F, x(s)=x(t)$ se e somente se existe $k \in \omega$ tal que $s=t \frown u_{k}$ ou $t=s \frown u_{k}$.

(10) Dados $s, t \in F$, se $s<<t$, então $x(s+t)=x(s) \cdot x(t)$.

(11) Dados $s, t \in F$, se $s<<t$, então $h(x(s+t))=h(x(s))+h(x(t))$.

(12) Para todo $n \in \omega, x\left[u_{n} \frown F\right]=X\left(u_{n}\right)$, de forma que, em particular, $x[F]=X$.

(13) Se houver $\left(W_{n}\right)_{n \geq 1}$ uma sequência previamente fixada de vizinhanças de e em $X$, é possível tomar $X\left(u_{n}\right)$ de forma que $X\left(u_{n+1}\right) \subset W_{n}$ para todo $n \in \mathbb{N}$.

Demonstração. Como $X$ é enumerável, tome uma ordem em $X$ isomorfa à ordem de $\omega$ (de forma que seja uma boa ordem).

Definiremos $x(t)$ e $X(t)$ por indução em $l(t)$.

Primeiramente, definimos $x(\varnothing)=e$ e $X(\varnothing)=X$. Usaremos a seguinte hipótese de indução: para algum $n \in \omega$, definimos $x(t)$ e $X(t)$ para todos $t \in F \operatorname{com} l(t) \leq n$ de forma que as condições (1) - (9) e (13) valham, e se $n \geq 1$, sequências $\left(a_{k}\right)_{0 \leq k \leq n-1}$ em $X$ e $\left(t_{k}\right)_{0 \leq k \leq n-1}$ em $F$ foram construídas de modo que valem as seguintes hipóteses adicionais, para cada $0 \leq k \leq n-1$ :

(i) $a_{k}=\min X \backslash\{x(t): t \in F$ e $l(t) \leq k\}$,

(ii) $a_{k} \in X\left(t_{k} \frown 1\right)$, e

(iii) se $h\left(a_{k}\right)=g_{p}\left(t_{k} \frown 1\right)$, então $a_{k}=x\left(t_{k} \frown 1\right)$.

Todas as condições são trivialmente satisfeitas no passo inicial $n=0$, exceto (8): para verificar essa, note que $e \in V(e)=X, \operatorname{logo} h(e)=h(e \cdot e)=h(e)+h(e), \operatorname{logo} h(x(\varnothing))=h(e)=[0]_{p}=g_{p}(\varnothing)$.

Para $n \geq 1$, começamos definindo $a_{n}=\min X \backslash\{x(t): t \in F$ e $l(t) \leq n\}$. Pela condição (3) e pelo fato de que $X(\varnothing)=X$, segue que os conjuntos $X(t) \operatorname{com} l(t)=n$ formam uma partição de $X$, logo existe um único $t_{n} \in F \operatorname{com} l\left(t_{n}\right)=n$ tal que $a_{n} \in X\left(t_{n}\right)$. Como $X\left(t_{n}\right)=x\left(t_{n}{ }^{\prime}\right) \cdot X\left(t_{n}{ }^{*}\right)$, tome $c_{n} \in X\left(t_{n}{ }^{*}\right)$ tal que $a_{n}=x\left(t_{n}{ }^{\prime}\right) \cdot c_{n}$.

Agora, para cada $s \in\left\{s_{i}^{n}: 0 \leq i \leq n\right\}$, tome $b_{s} \in X(s)$ tal que $b_{s} \neq x(s)$ e $h\left(b_{s}\right)=g_{p}(s \frown 1)$; tal $b_{s}$ existe pois, por (1) e (2), $X(s)$, é um aberto não-vazio de $X$, e como $X$ não possui pontos isolados, 
$X(s) \backslash\{x(s)\}$ é um aberto também não-vazio de $X$, e portanto $h[X(s) \backslash\{x(s)\}]=\mathbb{Z}_{p}$. No caso específico de $s=t_{n}{ }^{*}$, se $h\left(c_{n}\right)=g_{p}\left(t_{n}{ }^{*} 1\right)$, definimos $b_{t_{n}}{ }=c_{n}$; isso é possível pois se $c_{n}=x\left(t_{n}{ }^{*}\right)$, então por $(5), a_{n}=x\left(t_{n}{ }^{\prime}\right) \cdot c_{n}=x\left(t_{n}{ }^{\prime}\right) \cdot x\left(t_{n}{ }^{*}\right)=x\left(t_{n}\right)$, contradizendo a definição de $a_{n}$.

Assim, para cada $s \in\left\{s_{i}^{n}: 0 \leq i \leq n\right\}$, defina $x(s \frown 0)=x(s)$ e $x(s \frown 1)=b_{s}$. Note que $s^{\prime}=\varnothing \mathrm{e}$ $s^{*}=s$ pela definição, $\operatorname{logo} x\left(s^{\frown} 1\right)=x\left(s^{\prime}\right) \cdot x\left(s^{* \frown 1)}\right.$.

Dado $t \in F \operatorname{com} l(t)=n$, note que já definimos $x\left(t^{*} \frown 1\right) \in X\left(t^{*}\right)$ pois $t^{*} \in\left\{s_{i}^{n}: 0 \leq i \leq n\right\}$. Defina então $x\left(t^{\frown} 0\right)=x(t)$ e $x\left(t^{\frown} 1\right)=x\left(t^{\prime}\right) \cdot x\left(t^{*} \frown 1\right)$. Observe que $x(t)=x\left(t^{\prime}\right) \cdot x\left(t^{*}\right) \neq x\left(t^{\prime}\right) \cdot x\left(t^{* \frown 1)}=\right.$ $x\left(t^{\frown} 1\right)$, e que $x\left(t^{\frown} 1\right) \in x\left(t^{\prime}\right) \cdot X\left(t^{*}\right)=X(t)$. Além disso, por indução, $x\left(u_{n+1}\right)=x\left(u_{n} \frown 0\right)=$ $x\left(u_{n}\right)=x(\varnothing)=e$.

Tomaremos agora uma vizinhança clopen $U_{n}$ de $e$ em $G$ satisfazendo certas condições. Será possível tomar tal $U_{n}$ notando que é um número finito de condições, cada uma garantida pela existência de uma certa vizinhança de $e$, e que as condições não são afetadas ao se tomar a interseção de tais vizinhanças. E a existência de cada uma dessas vizinhanças é garantida ao se usar que a topologia de $G$ é Hausdorff, zero-dimensional, e invariante à esquerda (ou seja, as translações à esquerda são homeomorfismos). As condições são:

- $U_{n} \cap X \subset V(x(v))$ para todo $v \in F \operatorname{com} l(v) \leq n$ (lembre que $V(x(v))$ é vizinhança de $e$ em $\left.X\right)$;

- $x(v) \cdot U_{n} \cap X \subset x(v) \cdot X$ para todo $v \in F \operatorname{com} l(v) \leq n$ (lembre que $x(v) \cdot X \cap X$ é vizinhança de $x(v)$ em $X)$;

$-a_{n} \notin x\left(t_{n}\right) \cdot U_{n}\left(\right.$ pois $\left.a_{n} \neq x\left(t_{n}\right)\right)$

- $x(t) \cdot U_{n} \cap X \subset X(t)$ para todo $t \in F \operatorname{com} l(t)=n$ (pois $x(t) \cdot X \cap X$ e $X(t)$ são vizinhanças de $x(t)$ em $X)$;

$-x(t \frown 1) \notin x(t) \cdot U_{n}($ pois $x(t \frown 1) \neq x(t))$

- se houver $\left(W_{n}\right)_{n \geq 1}$ uma sequência previamente fixada de vizinhanças de $e$ em $X, U_{n} \cap X \subset W_{n}$.

Ser-nos-á útil mostrar que para todo $v \in F \operatorname{com} l(v) \leq n$, vale que $x(v) \cdot U_{n} \cap X=x(v) \cdot\left(U_{n} \cap X\right)$. Pela construção, $x(v) \cdot U_{n} \cap X \subset x(v) \cdot X, \operatorname{logo} x(v) \cdot U_{n} \cap X \subset\left(x(v) \cdot U_{n}\right) \cap(x(v) \cdot X)=x(v) \cdot\left(U_{n} \cap X\right)$. Por outro lado, $U_{n} \cap X \subset V(x(v))$, logo $x(v) \cdot\left(U_{n} \cap X\right) \subset x(v) \cdot V(x(v)) \subset X$, do que $x(v) \cdot\left(U_{n} \cap X\right) \subset$ $x(v) \cdot U_{n} \cap X$.

Definimos então $X(t \frown 0)=x(t) \cdot U_{n} \cap X$ e $X(t \frown 1)=X(t) \backslash X(t \frown 0)$. Agora, iremos verificar que as condições (1) - (9), (13), e também as hipóteses (i),(ii),(iii), valem para elementos de $F$ de comprimento $n+1$. Seja então $t \in F \operatorname{com} l(t)=n$. Defina $v=t \frown 1$ e $w=t \frown 0$. Como observado acima, $v^{\prime}=t^{\prime}, v^{*}=t^{*} 1, w^{*}=u_{n+1}$ e para algum $r \in \omega, t=\left(w^{\prime}\right) \frown\left(u_{r}\right)$, de forma que $x(t)=x\left(w^{\prime}\right)$ pela construção. 
Condição (1): $X(t)$ é clopen de $X$ por hipótese, $U_{n}$ é clopen e a topologia é invariante à esquerda, $\operatorname{logo} x(t) \cdot U_{n} \cap X$ é clopen de $X$. Como $x(t) \cdot U_{n} \cap X \subset X(t), X(t) \backslash X\left(t^{\frown} 0\right)=X\left(t^{\frown} 1\right)$ também é clopen.

Condição (2): Temos que $x(t \frown 0)=x(t) \in x(t) \cdot U_{n} \cap X=X(t \frown 0)$. Temos que $x(t \frown 1) \notin x(t) \cdot U_{n}$ por construção e $x\left(t^{\frown} 1\right) \in X(t), \operatorname{logo} x\left(t^{\frown} 1\right) \in X(t) \backslash\left(x(t) \cdot U_{n} \cap X\right)=X(t) \backslash X\left(t^{\frown} 0\right)=X\left(t^{\frown} 1\right)$.

Condição (3): Pela definição, $X(t \frown 0) \subset X(t)$ e $X\left(t^{\frown} 1\right)=X(t) \backslash X\left(t^{\frown} 0\right), \log o X(t \frown 0) \cup X(t \frown 1)=$ $X(t)$ e $X\left(t^{\frown} 0\right) \cap X\left(t^{\frown} 1\right)=\varnothing$.

Condição (4): Definimos que $x\left(t^{\frown} 0\right)=x(t)$.

Condição (5): Vale que $x(v)=x\left(t^{\frown} 1\right)=x\left(t^{\prime}\right) \cdot x\left(t^{*} \frown 1\right)=x\left(v^{\prime}\right) \cdot x\left(v^{*}\right) . \mathrm{E} x(w)=x(t)=x\left(w^{\prime}\right)=$ $x\left(w^{\prime}\right) \cdot e=x\left(w^{\prime}\right) \cdot x\left(w^{*}\right)$.

Condição (6): Vale que $X(w)=X\left(t^{\frown} 0\right)=x(t) \cdot U_{n} \cap X=x(t) \cdot\left(U_{n} \cap X\right)=x\left(w^{\prime}\right) \cdot X\left(u_{n+1}\right)=x\left(w^{\prime}\right)$. $X\left(w^{*}\right)$. $\mathrm{E} X(v)=X\left(t^{\frown} 1\right)=X(t) \backslash X\left(t^{\frown} 0\right)=X(t) \backslash\left(x(t) \cdot U_{n} \cap X\right)=X(t) \backslash\left(x(t) \cdot\left(U_{n} \cap X\right)\right)=$ $x\left(t^{\prime}\right) \cdot X\left(t^{*}\right) \backslash\left(x\left(t^{\prime}\right) \cdot x\left(t^{*}\right) \cdot\left(U_{n} \cap X\right)\right)=x\left(t^{\prime}\right) \cdot\left(X\left(t^{*}\right) \backslash x\left(t^{*}\right) \cdot\left(U_{n} \cap X\right)\right)=x\left(t^{\prime}\right) \cdot\left(X\left(t^{*}\right) \backslash\left(x\left(t^{*}\right) \cdot U_{n} \cap X\right)\right)=$ $x\left(t^{\prime}\right) \cdot X\left(t^{*} 1\right)=x\left(v^{\prime}\right) \cdot X\left(v^{*}\right)$.

Condição (7): Temos que $X\left(w^{*}\right)=X\left(u_{n+1}\right)=U_{n} \cap X \subset V(x(t))=V\left(x\left(w^{\prime}\right)\right)$. $\mathrm{E} X\left(v^{*}\right)=$ $X\left(t^{*} 1\right) \subset X\left(t^{*}\right) \subset V\left(x\left(t^{\prime}\right)\right)=V\left(x\left(v^{\prime}\right)\right)$.

Condição (8): Vale que $h(x(w))=h(x(t))=g_{p}(t)$, e como $w=t \frown 0$, então $g_{p}(w)=g_{p}(t)$. Por outro lado, temos que $h(x(v))=h\left(x\left(v^{\prime}\right) \cdot x\left(v^{*}\right)\right)=h\left(x\left(v^{\prime}\right)\right)+h\left(x\left(v^{*}\right)\right)$, pois $x\left(v^{*}\right) \in X\left(v^{*}\right) \subset$ $V\left(x\left(v^{\prime}\right)\right)$. Além disso, $x\left(v^{*}\right)=x\left(t^{*} 1\right)=b_{t^{*}}$, já que $t^{*} \in\left\{s_{i}^{n}: i \in n+1\right\}$; como por construção $h\left(b_{t^{*}}\right)=g_{p}\left(t^{*} \frown 1\right)$, segue que $h(x(v))=h\left(x\left(v^{\prime}\right)\right)+h\left(x\left(v^{*}\right)\right)=g_{p}\left(v^{\prime}\right)+h\left(b_{t^{*}}\right)=g_{p}\left(v^{\prime}\right)+g_{p}\left(t^{*} \frown 1\right)=$ $g_{p}\left(v^{\prime}\right)+g_{p}\left(v^{*}\right)$. Como $\operatorname{supp}\left(v^{\prime}\right) \cap \operatorname{supp}\left(v^{*}\right)=\varnothing$, vale que $g_{p}\left(v^{\prime}\right)+g_{p}\left(v^{*}\right)=g_{p}\left(v^{\prime}+v^{*}\right)=g_{p}(v)$.

Condição (9): Sem perda de generalidade, $l(s) \leq l(t)$. Se para algum $k \in \omega, t=s \frown u_{k}$, então por (4), $x(s)=x(t)$. Caso contrário, então $s$ possui um 1 onde $t$ possui um 0 ; ou $t$ possui um 1 onde $s$ possui um zero, ou onde $s$ não está definido; de qualquer forma, pelo item (3) $x(s)$ e $x(t)$ pertencem a clopens disjuntos de $X, \log 0 x(s) \neq x(t)$.

Condição (13): Se houver $\left(W_{n}\right)_{n \geq 1}$ uma sequência previamente fixada de vizinhanças de $e$ em $X$, vale que $X\left(u_{n+1}\right)=U_{n} \cap X \subset W_{n}$.

Hipótese (i): Por definição, $a_{n}=\min X \backslash\{x(t): t \in F$ e $l(t) \leq n\}$.

Hipótese (ii): Vale que $a_{n} \notin x\left(t_{n}\right) \cdot U_{n}$, $\operatorname{logo} a_{n} \notin X\left(t_{n} \frown 0\right)$. Como $a_{n} \in X\left(t_{n}\right)$, resta que $a_{n} \in X\left(t_{n} \frown 1\right)$.

Hipótese (iii): Suponha que $h\left(a_{n}\right)=g_{p}\left(t_{n} \frown 1\right)$. Como $t_{n} \frown 1=t_{n}{ }^{\prime}+t_{n}{ }^{* \frown 1}$, e estes dois têm suportes disjuntos, vale que $g_{p}\left(t_{n} \frown 1\right)=g_{p}\left(t_{n}{ }^{\prime}\right)+g_{p}\left(t_{n}{ }^{* \frown 1}\right)$. Lembre que $a_{n}=x\left(t_{n}{ }^{\prime}\right) \cdot c_{n}$, com $c_{n} \in X\left(t_{n}{ }^{*}\right) \subset V\left(x\left(t_{n}{ }^{\prime}\right)\right)$, de forma que $h\left(a_{n}\right)=h\left(x\left(t_{n}{ }^{\prime}\right)\right)+h\left(c_{n}\right)=g_{p}\left(t_{n}{ }^{\prime}\right)+h\left(c_{n}\right)$. Segue que $h\left(c_{n}\right)=g_{p}\left(t_{n}{ }^{* \frown 1}\right)$. Por construção, nesse caso $c_{n}=b_{t_{n}{ }^{*}}, \operatorname{logo} c_{n}=x\left(t_{n}{ }^{* \frown 1}\right)$, e portanto $a_{n}=x\left(t_{n}{ }^{\prime}\right) \cdot x\left(t_{n}{ }^{* \frown 1}\right)=x\left(\left(t_{n} \frown 1\right)^{\prime}\right) \cdot x\left(\left(t_{n} \frown 1\right)^{*}\right)=x\left(t_{n} \frown 1\right)$. 
Assim, a indução está completa. Resta provar que valem (10), (11) e (12) sabendo que valem (1) - (9), (13), e (i),(ii),(iii).

Condição (10): Primeiramente, mostraremos por indução em $k$ que se a representação canônica de $s$ é $s_{i_{0}}^{m_{0}}+\cdots+s_{i_{k}}^{m_{k}}$, então $x(s)=x\left(s_{i_{0}}^{m_{0}}\right) \cdot \ldots \cdot x\left(s_{i_{k}}^{m_{k}}\right)$. Para $k=0$, temos que $s=s_{i_{0}}^{m_{0}}$, logo $x(s)=x\left(s_{i_{0}}^{m_{0}}\right)$. Se $s=s_{i_{0}}^{m_{0}}+\cdots+s_{i_{k}}^{m_{k}}+s_{i_{k+1}}^{m_{k+1}}$, como $s^{\prime}=s_{i_{0}}^{m_{0}}+\cdots+s_{i_{k}}^{m_{k}}$ e $s^{*}=s_{i_{k+1}}^{m_{k+1}}$, usando (5), $x(s)=x\left(s^{\prime}\right) \cdot x\left(s^{*}\right)=x\left(s_{i_{0}}^{m_{0}}\right) \cdot \ldots \cdot x\left(s_{i_{k}}^{m_{k}}\right) \cdot x\left(s_{i_{k+1}}^{m_{k+1}}\right)$.

Assim, dados $s=s_{i_{0}}^{m_{0}}+\cdots+s_{i_{k}}^{m_{k}}$ e $t=s_{j_{0}}^{n_{0}}+\cdots+s_{j_{l}}^{n_{l}}$, com $s<<t$, verifiquemos caso a caso: se $i_{k}<m_{k}, s+t=s_{i_{0}}^{m_{0}}+\cdots+s_{i_{k}}^{m_{k}}+s_{j_{0}}^{n_{0}}+\cdots+s_{j_{l}}^{n_{l}}$ é a representação canônica, então $x(s+t)=x\left(s_{i_{0}}^{m_{0}}\right) \cdots$. $x\left(s_{i_{k}}^{m_{k}}\right) \cdot x\left(s_{j_{0}}^{n_{0}}\right) \cdot \ldots \cdot x\left(s_{j_{l}}^{n_{l}}\right)=x(s) \cdot x(t) ;$ se $i_{k}=m_{k}$ e $m_{k} \leq n_{l}, s+t=s_{i_{0}}^{m_{0}}+\cdots+s_{i_{k-1}}^{m_{k-1}}+s_{j_{0}}^{n_{0}}+\cdots+s_{j_{l}}^{n_{l}}$ é a representação canônica, então $x(s+t)=x\left(s_{i_{0}}^{m_{0}}\right) \cdot \ldots \cdot x\left(s_{i_{k-1}}^{m_{k-1}}\right) \cdot x\left(s_{j_{0}}^{n_{0}}\right) \cdot \ldots \cdot x\left(s_{j_{l}}^{n_{l}}\right)=x\left(s_{i_{0}}^{m_{0}}\right)$. $\ldots \cdot x\left(s_{i_{k-1}}^{m_{k-1}}\right) \cdot e \cdot x\left(s_{j_{0}}^{n_{0}}\right) \cdot \ldots \cdot x\left(s_{j_{l}}^{n_{l}}\right)=x\left(s_{i_{0}}^{m_{0}}\right) \cdot \ldots \cdot x\left(s_{i_{k-1}}^{m_{k-1}}\right) \cdot x\left(s_{m_{k}}^{m_{k}}\right) \cdot x\left(s_{j_{0}}^{n_{0}}\right) \cdot \ldots \cdot x\left(s_{j_{l}}^{n_{l}}\right)=x(s) \cdot x(t) ;$ se $i_{k}=m_{k}$ e $m_{k}>n_{l}, s+t=s_{i_{0}}^{m_{0}}+\cdots+s_{i_{k-1}}^{m_{k-1}}+s_{j_{0}}^{n_{0}}+\cdots+s_{j_{l}}^{n_{l}}+s_{m_{k}}^{m_{k}}$ é a representação canônica, $\operatorname{logo} x(s+t)=x\left(s_{i_{0}}^{m_{0}}\right) \cdot \ldots \cdot x\left(s_{i_{k-1}}^{m_{k-1}}\right) \cdot x\left(s_{j_{0}}^{n_{0}}\right) \cdot \ldots \cdot x\left(s_{j_{l}}^{n_{l}}\right) \cdot x\left(s_{m_{k}}^{m_{k}}\right)=x\left(s_{i_{0}}^{m_{0}}\right) \cdot \ldots \cdot x\left(s_{i_{k-1}}^{m_{k-1}}\right) \cdot e \cdot x\left(s_{j_{0}}^{n_{0}}\right) \cdot$ $\ldots \cdot x\left(s_{j_{l}}^{n_{l}}\right) \cdot e=x\left(s_{i_{0}}^{m_{0}}\right) \cdot \ldots \cdot x\left(s_{i_{k-1}}^{m_{k-1}}\right) \cdot x\left(s_{m_{k}}^{m_{k}}\right) \cdot x\left(s_{j_{0}}^{n_{0}}\right) \cdot \ldots \cdot x\left(s_{j_{l}}^{n_{l}}\right)=x(s) \cdot x(t)$.

Condição (11): Primeiramente, mostraremos por indução em $k$ que se a representação canônica de $s$ é $s_{i_{0}}^{m_{0}}+\cdots+s_{i_{k}}^{m_{k}}$, então $h(x(s))=h\left(x\left(s_{i_{0}}^{m_{0}}\right)\right)+\cdots+h\left(x\left(s_{i_{k}}^{m_{k}}\right)\right)$.Para $k=0$, temos que $s=s_{i_{0}}^{m_{0}}, \operatorname{logo} h(x(s))=h\left(x\left(s_{i_{0}}^{m_{0}}\right)\right)$. Se $s=s_{i_{0}}^{m_{0}}+\cdots+s_{i_{k}}^{m_{k}}+s_{i_{k+1}}^{m_{k+1}}$, como $s^{\prime}=s_{i_{0}}^{m_{0}}+\cdots+s_{i_{k}}^{m_{k}} \mathrm{e}$ $s^{*}=s_{i_{k+1}}^{m_{k+1}}$, usando que $x\left(s^{*}\right) \in X\left(s^{*}\right) \subset V\left(x\left(s^{\prime}\right)\right), h(x(s))=h\left(x\left(s^{\prime}\right)\right)+h\left(x\left(s^{*}\right)\right)=h\left(x\left(s_{i_{0}}^{m_{0}}\right)\right)+$ $\cdots+h\left(x\left(s_{i_{k}}^{m_{k}}\right)\right)+h\left(x\left(s_{i_{k+1}}^{m_{k+1}}\right)\right)$.

Assim, dados $s=s_{i_{0}}^{m_{0}}+\cdots+s_{i_{k}}^{m_{k}}$ e $t=s_{j_{0}}^{n_{0}}+\cdots+s_{j_{l}}^{n_{l}}$, com $s<<t$, verifiquemos caso a caso: se $i_{k}<m_{k}, s+t=s_{i_{0}}^{m_{0}}+\cdots+s_{i_{k}}^{m_{k}}+s_{j_{0}}^{n_{0}}+\cdots+s_{j_{l}}^{n_{l}}$ é a representação canônica, então $h(x(s+t))=h\left(x\left(s_{i_{0}}^{m_{0}}\right)\right)+\cdots+h\left(x\left(s_{i_{k}}^{m_{k}}\right)\right)+h\left(x\left(s_{j_{0}}^{n_{0}}\right)\right)+\cdots+h\left(x\left(s_{j_{l}}^{n_{l}}\right)\right)=h(x(s))+h(x(t)) ;$ se $i_{k}=m_{k}$ e $m_{k} \leq n_{l}, s+t=s_{i_{0}}^{m_{0}}+\cdots+s_{i_{k-1}}^{m_{k-1}}+s_{j_{0}}^{n_{0}}+\cdots+s_{j_{l}}^{n_{l}}$ é a representação canônica, então $h(x(s+t))=$ $h\left(x\left(s_{i_{0}}^{m_{0}}\right)\right)+\cdots+h\left(x\left(s_{i_{k-1}}^{m_{k-1}}\right)\right)+h\left(x\left(s_{j_{0}}^{n_{0}}\right)\right)+\cdots+h\left(x\left(s_{j_{l}}^{n_{l}}\right)\right)=h\left(x\left(s_{i_{0}}^{m_{0}}\right)\right)+\cdots+h\left(x\left(s_{i_{k-1}}^{m_{k-1}}\right)\right)+h(e)+$ $h\left(x\left(s_{j_{0}}^{n_{0}}\right)\right)+\cdots+h\left(x\left(s_{j_{l}}^{n_{l}}\right)\right)=h\left(x\left(s_{i_{0}}^{m_{0}}\right)\right)+\cdots+h\left(x\left(s_{i_{k-1}}^{m_{k-1}}\right)\right)+h\left(x\left(s_{m_{k}}^{m_{k}}\right)\right)+h\left(x\left(s_{j_{0}}^{n_{0}}\right)\right)+\cdots+h\left(x\left(s_{j_{l}}^{n_{l}}\right)\right)=$ $h(x(s))+h(x(t))$; se $i_{k}=m_{k}$ e $m_{k}>n_{l}, s+t=s_{i_{0}}^{m_{0}}+\cdots+s_{i_{k-1}}^{m_{k-1}}+s_{j_{0}}^{n_{0}}+\cdots+s_{j_{l}}^{n_{l}}+s_{m_{k}}^{m_{k}}$ é a representação canônica, $\operatorname{logo} h(x(s+t))=h\left(x\left(s_{i_{0}}^{m_{0}}\right)\right)+\cdots+h\left(x\left(s_{i_{k-1}}^{m_{k-1}}\right)\right)+h\left(x\left(s_{j_{0}}^{n_{0}}\right)\right)+\cdots+$ $h\left(x\left(s_{j_{l}}^{n_{l}}\right)\right)+h\left(x\left(s_{m_{k}}^{m_{k}}\right)\right)=h\left(x\left(s_{i_{0}}^{m_{0}}\right)\right)+\cdots+h\left(x\left(s_{i_{k-1}}^{m_{k-1}}\right)\right)+h(e)+h\left(x\left(s_{j_{0}}^{n_{0}}\right)\right)+\cdots+h\left(x\left(s_{j_{l}}^{n_{l}}\right)\right)+h(e)=$ $h\left(x\left(s_{i_{0}}^{m_{0}}\right)\right)+\cdots+h\left(x\left(s_{i_{k-1}}^{m_{k-1}}\right)\right)+h\left(x\left(s_{m_{k}}^{m_{k}}\right)\right)+h\left(x\left(s_{j_{0}}^{n_{0}}\right)\right)+\cdots+h\left(x\left(s_{j_{l}}^{n_{l}}\right)\right)=h(x(s))+h(x(t))$.

Condição (12): primeiro, mostraremos que dado $a \in X$, existe $v \in F$ tal que $a=x(v)$. Suponha, por absurdo, que $X \backslash x[F] \neq \varnothing$ e seja $a=\min X \backslash x[F]$.

Afirmação: para algum $n \in \omega, a=a_{n}$. Para ver isso, denote $F_{n}:=\{t \in F: x(t) \leq n\}$. Vale que $F=\bigcup_{n \in \omega} F_{n}$. Como $F_{n} \subset F_{n+1} \subset F$, para todo $n \in \omega$, segue que $X \backslash x\left[F_{n}\right] \supset X \backslash x\left[F_{n+1}\right] \supset$ 
$X \backslash x[F]$, para todo $n \in \omega$, e assim $\min X \backslash x\left[F_{n}\right] \leq \min X \backslash x\left[F_{n+1}\right] \leq \min X \backslash x[F]$. Portanto, $\left(\min X \backslash x\left[F_{n}\right]\right)_{n \geq 0}$ é uma sequência crescente e limitada em uma ordem de tipo $\omega$, logo estaciona: tome $n \in \omega$ tal que $\min X \backslash x\left[F_{n}\right]=\min X \backslash x\left[F_{n+1}\right]=\min X \backslash x\left[F_{n+2}\right]=\cdots$. Vale então que $\min X \backslash x\left[F_{n}\right]=\min X \backslash x[F]$ : se valesse que $\min X \backslash x\left[F_{n}\right]<\min X \backslash x[F]$, então para algum $m>n$, $\min X \backslash x[F] \in x\left[F_{m}\right]$, e portanto $\min X \backslash x\left[F_{n}\right]<\min X \backslash x\left[F_{m}\right]$, o que não vale pela escolha de $n$. Portanto, $a=\min X \backslash x[F]=\min X \backslash x\left[F_{n}\right]=a_{n}$.

Tome $m \in\{0, \ldots, p-1\}$ tal que $h\left(a_{n}\right)=g_{p}\left(t_{n} \frown 1\right)+[m]_{p}$. Caso $m=0$, por (iii) teríamos que $a=a_{n}=x\left(t_{n} \frown 1\right) ; \operatorname{logo} m \geq 1$. Agora, seja $k \geq 0$ e suponha que $h\left(a_{n+k}\right)=g_{p}\left(t_{n+k} \frown 1\right)+[m]_{p}-[k]_{p} ;$ note que isso é satisfeito quando $k=0$. Por (i), e pelo que foi visto acima, $a_{n+k+1}=a_{n+k}$. Por (ii), $a_{n+k} \in X\left(t_{n+k} \frown 1\right)$ e $a_{n+k+1} \in X\left(t_{n+k+1} \frown 1\right) \subset X\left(t_{n+k+1}\right)$. Como ambos $t_{n+k} \frown 1 \mathrm{e}$ $t_{n+k+1}$ são de comprimento $n+k+1$, segue que $t_{n+k} \frown 1=t_{n+k+1}$. Dessa forma, $g_{p}\left(t_{n+k+1} \frown 1\right)=$ $g_{p}\left(t_{n+k+1}\right)+[1]_{p}=g_{p}\left(t_{n+k} \frown 1\right)+[1]_{p}, \operatorname{logo} g_{p}\left(t_{n+k} \frown 1\right)=g_{p}\left(t_{n+k+1} \frown 1\right)-[1]_{p} ;$ portanto, temos que $h\left(a_{n+k+1}\right)=h\left(a_{n+k}\right)=g_{p}\left(t_{n+k} \frown 1\right)+[m]_{p}-[k]_{p}=g_{p}\left(t_{n+k+1} \frown 1\right)-[1]_{p}+[m]_{p}-[k]_{p}=$ $g_{p}\left(t_{n+k+1} \frown 1\right)+[m]_{p}-[k+1]_{p}$. Assim, vale a indução. Em particular, quando $k=m, h\left(a_{n+m}\right)=$ $g_{p}\left(t_{n+m} \frown 1\right)+[m]_{p}-[m]_{p}=g_{p}\left(t_{n+m} \frown 1\right)$; logo por (iii), $a=a_{n+m}=x\left(t_{n+m} \frown 1\right)$, uma contradição, pois $a \in X \backslash x[F]$.

Dessa forma, $x[F]=X$.

Agora, para terminar o (12), note que para todo $n \in \omega$ e todo $t \in F$, vale que $x\left(u_{n} \frown t\right) \in X\left(u_{n} \frown t\right) \subset$ $X\left(u_{n}\right)$, de forma que $x\left[u_{n} \frown F\right] \subset X\left(u_{n}\right)$. Resta ver que $X \backslash x\left[u_{n} \frown F\right] \subset X \backslash X\left(u_{n}\right)$. Seja $a \in$ $X \backslash x\left[u_{n} \frown F\right]$. Como $X=x[F]$, tome $v \in F \backslash u_{n} \frown F$ tal que $a=x(v)$. Como $e=x\left(u_{n}\right)=$ $x\left(u_{n} \frown \varnothing\right) \in x\left[u_{n} \frown F\right]$, então $a \neq e$, e portanto $v \notin\left\{u_{m}: m \in \omega\right\}$, e $\operatorname{assim} \operatorname{supp}(v) \neq \varnothing$. Seja $k=\min \operatorname{supp}(v)$. Como $v \in F \backslash u_{n} \frown F$, então $k \leq n$. Vale que $x(v) \in X\left(s_{k-1}^{k}\right)$, pois $s_{k-1}^{k}$ é um trecho inicial de $v$, e que $X\left(u_{n}\right) \subset X\left(u_{k}\right)$, pois $n \geq k$. Por fim, como $u_{k}=u_{k-1} \frown 0$ e $s_{k-1}^{k}=u_{k-1} \frown 1$, então $X\left(s_{k-1}^{k}\right) \cap X\left(u_{k}\right)=\varnothing, \operatorname{logo} a=x(v) \notin X\left(u_{n}\right)$. Segue que $X \backslash x\left[u_{n} \frown F\right] \subset X \backslash X\left(u_{n}\right)$ como afirmamos.

Atenção: A partir daqui e até a enunciação do Teorema de Zelenyuk, $G$ será um grupo discreto com identidade $e$ e $C$ será um subsemigrupo finito de $G^{*}$.

Definição 3.39. (a) $C^{\sim}=\{x \in \beta G: x \cdot C \subset C\}$.

(b) $\varphi$ é o filtro dos subconjuntos $U$ de $G$ tais que $C \subset \bar{U}$.

(c) $\varphi^{\sim}$ é o filtro dos subconjuntos $U$ de $G$ tais que $C^{\sim} \subset \bar{U}$.

Observe que $C^{\sim}$ é um semigrupo, e que de fato $\varphi$ e $\varphi^{\sim}$ são filtros. De fato, vale que $\varphi=\bigcap C$ e $\varphi^{\sim}=\bigcap C^{\sim}$, pois: $U \in \varphi \Leftrightarrow C \subset \bar{U} \Leftrightarrow$ para todo $c \in C, c \in \bar{U} \Leftrightarrow$ para todo $c \in C$, $U \in c \Leftrightarrow U \in \bigcap C$. Analogamente, $\varphi^{\sim}=\bigcap C^{\sim}$. 
Lema 3.40. É possivel definir uma topologia invariante à esquerda em $G$ tal que $\varphi^{\sim}$ é o filtro das vizinhanças de e. Se valer que $x \cdot C=C$ para todo $x \in C^{\sim}$, então esta topologia tem uma base de clopens.

Demonstração. Para cada $U \in \varphi$, defina $U^{\wedge}=\{a \in G: a \cdot C \subset \bar{U}\}$. Note que $e \in U^{\wedge}$. Mostraremos que

(i) para cada $U \in \varphi, U^{\wedge} \in \varphi^{\sim}$,

(ii) $\left\{U^{\wedge}: U \in \varphi\right\}$ é uma base para o filtro $\varphi^{\sim}$, e

(iii) para todo $U \in \varphi$ e todo $a \in U^{\wedge}, a^{-1} U^{\wedge} \in \varphi^{\sim}$.

Verificando (i): suponha que para um certo $U \in \varphi, C^{\sim} \not \subset \overline{U^{\wedge}}$. Tome $x \in C^{\sim} \backslash \overline{U^{\wedge}}$. Como $U^{\wedge} \notin x$, então $G \backslash U^{\wedge} \in x$. Mas $G \backslash U^{\wedge}=\{a \in G: a \cdot C \not \subset \bar{U}\}=\{a \in G$ : existe $y \in C$ tal que $a \cdot y \notin \bar{U}\}=$ $\bigcup_{y \in C}\{a \in G: U \notin a \cdot y\}=\bigcup_{y \in C}\left\{a \in G: a^{-1} U \notin y\right\}$. Como $C$ é finito, vale que para algum $y \in C$, $\left\{a \in G: a^{-1} U \notin y\right\} \in x, \operatorname{logo}\left\{a \in G: a^{-1} U \in y\right\} \notin x$, e assim $U \notin x \cdot y$, ou seja, $x \cdot y \notin \bar{U}$. Porém, $x \in C^{\sim}$ e $y \in C, \operatorname{logo} x \cdot y \in C \subset \bar{U}$. Contradição. Logo, $U^{\wedge} \in \varphi^{\sim}$.

Verificando (ii): O item acima nos diz que $\left\{U^{\wedge}: U \in \varphi\right\} \subset \varphi^{\sim}$. Dados $U, V \in \varphi,(U \cap V)^{\wedge} \in \varphi^{\sim}$ (pois $U \cap V \in \varphi$ ), e $(U \cap V)^{\wedge}=\{a \in G: a \cdot C \subset \overline{U \cap V}\}=\{a \in G: a \cdot C \subset \bar{U} \cap \bar{V}\}=U^{\wedge} \cap V^{\wedge}$, de forma que $\left\{U^{\wedge}: U \in \varphi\right\}$ é fechado para interseções finitas. Agora, resta ver que para todo $V \in \varphi^{\sim}$ existe $U \in \varphi$ tal que $U^{\wedge} \subset V$.

Afirmação: Basta ver que $\bigcap_{U \in \varphi} \overline{U^{\wedge}}=C^{\sim}$. Pois suponha que exista $V \in \varphi^{\sim}$ tal que para todo $U \in \varphi, U^{\wedge} \backslash V \neq \varnothing$. Valeria que $\left\{U^{\wedge} \backslash V: U \in \varphi\right\}$ seria uma coleção com a p.i.f., logo existiria $x \in \bigcap_{U \in \varphi} \overline{U^{\wedge} \backslash V}$. Ter-se-ia então que $x \in \bigcap_{U \in \varphi} \overline{U^{\wedge} \backslash V} \subset \bigcap_{U \in \varphi} \overline{U^{\wedge}}=C^{\sim} \subset \bar{V}$, ou seja, $x \in \bar{V}$, uma contradição.

Provemos então que $\bigcap_{U \in \varphi} \overline{U^{\wedge}}=C^{\sim}$. Vale que $\bigcap_{U \in \varphi} \overline{U^{\wedge}} \supset C^{\sim}$, já que $\left\{U^{\wedge}: U \in \varphi\right\} \subset \varphi^{\sim}$ e pela definição de $\varphi^{\sim}$. Agora, suponha por absurdo que existe $x \in \bigcap_{U \in \varphi} \overline{U^{\wedge} \backslash C^{\sim}}$. $x \notin C^{\sim}$, ou seja, existe $y \in C$ tal que $x \cdot y \notin C$. Como $C$ é finito e $\beta G$ é Hausdorff, $C$ é fechado, logo existe $A \subset G$ tal que $x \cdot y \in \bar{A}$ e $C \cap \bar{A}=\varnothing$. Logo $C \subset \overline{G \backslash A}$, ou seja, $G \backslash A \in \varphi . x \cdot y \notin \overline{G \backslash A}$, $\operatorname{logo}$ $\left\{a \in G: a^{-1}(G \backslash A) \in y\right\} \notin x$, ou seja, $\left\{a \in G: a^{-1}(G \backslash A) \notin y\right\}=\{a \in G: a \cdot y \notin \overline{G \backslash A}\} \in x$. Como $(G \backslash A)^{\wedge}=\{a \in G: a \cdot C \subset \overline{G \backslash A}\}$, então $(G \backslash A)^{\wedge} \cap\{a \in G: a \cdot y \notin \overline{G \backslash A}\}=\varnothing$, e portanto $(G \backslash A)^{\wedge} \notin x$, ou seja $x \notin \overline{(G \backslash A)^{\wedge}}$. Absurdo, pois $G \backslash A \in \varphi$.

Verificando (iii): sejam $U \in \varphi$ e $a \in U^{\wedge}$, e suponha que $a^{-1} U^{\wedge} \notin \varphi^{\sim}$. Logo existe $y \in C^{\sim} \overline{a^{-1} U^{\wedge}}$. Portanto, $G \backslash a^{-1} U^{\wedge}=\left\{b \in G: a \cdot b \notin U^{\wedge}\right\}=\{b \in G$ : existe $z \in C$ tal que $a \cdot b \cdot z \notin \bar{U}\} \in y$. Como $C$ é finito, tome $z \in C$ tal que $\{b \in G: a \cdot b \cdot z \notin \bar{U}\} \in y$. Segue que $a \cdot y \cdot z \notin \bar{U}$. Porém, $y \cdot z \in C$ e $a \in U^{\wedge}, \operatorname{logo} a \cdot y \cdot z \in \bar{U}$. Contradição.

Munido de (i), (ii) e (iii), defina $\mathcal{B}=\left\{a \cdot U^{\wedge}: U \in \varphi, a \in G\right\}$. Mostremos que $\mathcal{B}$ é uma base para 
uma topologia invariante à esquerda em $G$ tal que $\varphi^{\sim}$ é o filtro das vizinhanças de $e$.

Para ver que é base, sejam $U, V \in \varphi$ e $a, b, c \in G$, e suponha $c \in a \cdot U^{\wedge} \cap b \cdot V^{\wedge}$. Temos que $a^{-1} \cdot c \in U^{\wedge}$, $\operatorname{logo}$ por (iii) $c^{-1} \cdot a \cdot U^{\wedge} \in \varphi^{\sim}$, logo por (ii) existe $W_{1} \in \varphi$ tal que $W_{1}^{\wedge} \subset c^{-1} \cdot a \cdot U^{\wedge}$. Analogamente, existe $W_{2} \in \varphi$ tal que $W_{2}^{\wedge} \subset c^{-1} \cdot b \cdot V^{\wedge}$. Temos então que $W_{1}^{\wedge} \cap W_{2}^{\wedge} \subset c^{-1} \cdot a \cdot U^{\wedge} \cap c^{-1} \cdot b \cdot V^{\wedge}$, $\operatorname{logo} c \cdot\left(W_{1} \cap W_{2}\right)^{\wedge} \subset a \cdot U^{\wedge} \cap b \cdot V^{\wedge}$. Como $W_{1} \cap W_{2} \in \varphi$, vale que $e \in\left(W_{1} \cap W_{2}\right)^{\wedge}$, $\operatorname{logo}$ $c \in c \cdot\left(W_{1} \cap W_{2}\right)^{\wedge} \in \mathcal{B}$.

Como a base $\mathcal{B}$ é invariante à esquerda, então a topologia gerada por ela é invariante à esquerda.

Para ver que $\varphi^{\sim}$ é o filtro das vizinhanças de $e$, note por (ii) que cada membro de $\varphi^{\sim}$ é uma vizinhança de $e$ (pois $e \in U^{\wedge}=e \cdot U^{\wedge}$, para cada $U \in \varphi$ ). Por outro lado, dada $W$ vizinhança de $e$, tome $a \in G$ e $U \in \varphi$ tais que $e \in a \cdot U^{\wedge} \subset W$. Vale que $a^{-1}=a^{-1} \cdot e \in U^{\wedge}$, logo por (iii) $a \cdot U^{\wedge} \in \varphi^{\sim}$, do que $W \in \varphi^{\sim}$.

Finalmente, mostraremos que se $x \cdot C=C$ para todo $x \in C^{\sim}$, então para todo $U \in \varphi, U^{\wedge}$ é fechado. Disso seguirá que $\mathcal{B}$ é uma base de clopens, pois a topologia é invariante à esquerda. Sejam então $U \in \varphi$ e $a \in G \backslash U^{\wedge}$. Mostraremos que $V:=G \backslash a^{-1} \cdot U^{\wedge} \in \varphi^{\sim}$, logo $a \cdot V=G \backslash U^{\wedge}$ é uma vizinhança de $a$ disjunta de $U^{\wedge}$. Ou seja, devemos ver que $C^{\sim} \subset \bar{V}$. Seja $y \in C^{\sim}$. Como $a \notin U^{\sim}$, então $a \cdot(y \cdot C)=a \cdot C \not \subset \bar{U}$, logo tome $z \in C$ tal que $a \cdot y \cdot z \notin \bar{U}$. Assim, temos que $\{b \in G: a \cdot b \cdot z \notin \bar{U}\} \in y$ e $\{b \in G: a \cdot b \cdot z \notin \bar{U}\} \subset\{b \in G: a \cdot b \cdot C \not \subset \bar{U}\}=G \backslash\{b \in G: a \cdot b \cdot C \subset \bar{U}\}=G \backslash a^{-1} \cdot U^{\wedge}=V$. Logo $V \in y$, como queríamos.

Lema 3.41. Assuma que $x \cdot C=C$ para todo $x \in C^{\sim}$. Então são equivalentes:

(a) A topologia definida no Lema 3.40 é Hausdorff.

(b) $\{a \in G: a \cdot C \subset C\}=\{e\}$.

(c) $\cap \varphi^{\sim}=\{e\}$.

Demonstração. (a) $\Rightarrow$ (b): Por um lado, temos que $e \cdot C \subset C$. Por outro, seja $a \in G \backslash\{e\}$. Como $\varphi^{\sim}$ é o filtro das vizinhanças de $e$, tome $V \in \varphi^{\sim}$ tal que $a \notin V$. Como $C^{\sim} \subset \bar{V}$, então $a \notin C^{\sim}$, ou seja, $a \cdot C \not \subset C$.

(b) $\Rightarrow(\mathrm{c})$ : Como $\varphi^{\sim}$ é o filtro das vizinhanças de $e$, temos que $e \in \bigcap \varphi^{\sim}$. Por outro lado, tome $a \in G \backslash\{e\}$. Por hipótese, $a \cdot C \not \subset C$, ou seja, $a \notin C^{\sim}$. Agora, observe que $C^{\sim}=\{x \in \beta G: x \cdot C \subset$ $C\}=\{x \in \beta G: x \cdot y \in C$, para todo $y \in C\}=\bigcap_{y \in C}\{x \in \beta G: x \cdot y \in C\}=\bigcap_{y \in C}\{x \in \beta G$ : $\left.\rho_{y}(x) \in C\right\}=\bigcap_{y \in C} \rho_{y}{ }^{-1}[C], \operatorname{logo} C^{\sim}$ é fechado em $\beta G$. Assim, tome $A \subset G$ tal que $a \in A$ e $C^{\sim} \cap \bar{A}=\varnothing ; C^{\sim} \subset \overline{G \backslash A}$, ou seja, $G \backslash A \in \varphi^{\sim}$, e $a \notin G \backslash A$; portanto, $a \notin \cap \varphi^{\sim}$.

(c) $\Rightarrow$ (a): Seja $a \in G \backslash\{e\}$. Pela hipótese e pelo item (ii) do Lema 3.40, tome $U \in \varphi$ tal que $a \notin U^{\wedge}$; pela hipótese geral do Lema, $U^{\wedge}=: V$ é clopen, $\log o V$ e $G \backslash V$ são vizinhanças disjuntas de $e$ e $a$, respectivamente. 
Agora, dados $b, c \in G$ distintos, pelo que provamos acima existe $V$ vizinhança clopen de $e$ tal que $b^{-1} \cdot c \in G \backslash V$. Logo $b \cdot V$ e $b \cdot(G \backslash V)=G \backslash b \cdot V$ são vizinhanças disjuntas de $b$ e $c$, respectivamente.

Atenção: A partir daqui e até a enunciação do Teorema de Zelenyuk, $G$ será um grupo discreto com identidade $e$ e $C$ será um subgrupo finito de $G^{*}$, com identidade $u$. (Na p. 92 exigíramos somente que $C$ fosse um subsemigrupo.)

Observe que isto implica que $x \cdot C=C$ para todo $x \in C^{\sim}$, pois: dado $c \in C, x \cdot u \in C$, $\operatorname{logo}$ possui uma inversa $(x \cdot u)^{-1} \in C$. Assim, $x \cdot u \cdot(x \cdot u)^{-1} \cdot c \in x \cdot C$, e $x \cdot u \cdot(x \cdot u)^{-1} \cdot c=u \cdot c=c$.

Lema 3.42. Se $G$ não possui grupos finitos não-triviais, então $\bigcap \varphi^{\sim}=\{e\}$.

Demonstração. Vale que $\bigcap \varphi^{\sim}=C^{\sim} \cap G$, pois: dado $a \in C^{\sim} \cap G$, para cada $U \in \varphi^{\sim}, a \in U$, logo $a \in \bigcap \varphi^{\sim}$; e se $a \in G \backslash C^{\sim}$, então $a \neq p$ para todo $p \in C^{\sim}$, de forma que $G \backslash\{a\} \in p$ para todo $p \in C^{\sim}$, ou seja, $C^{\sim} \subset \overline{G \backslash\{a\}}$, ou seja, $G \backslash\{a\} \in \varphi^{\sim}$, de forma que $a \notin \bigcap \varphi^{\sim}$.

Assim, $\cap \varphi^{\sim}=C^{\sim} \cap G=\{a \in G: a \cdot C=C\}$. Agora, dados $a, b \in C^{\sim} \cap G$, temos que $a \cdot b \cdot C=a \cdot C=C, \operatorname{logo} a \cdot b \in C^{\sim} \cap G ;$ e $a^{-1} \cdot C=a^{-1} \cdot(a \cdot C)=C, \operatorname{logo} a^{-1} \in C^{\sim} \cap G$. Portanto, $C^{\sim} \cap G$ é um subgrupo de de $G$. Pela hipótese, ele é infinito, ou é $\{e\}$. Porém, se fosse infinito, como $a \cdot u, b \cdot u \in C$ para todos $a, b \in C^{\sim} \cap G$, e $C$ é finito, existiriam $a, b \in G$ distintos tais que $a \cdot u=b \cdot u$, contrariando o Lema 3.8.

Assim, com os Lemas 3.40, 3.41 e 3.42, temos o seguinte:

Lema 3.43. Existe uma topologia em $G$ invariante à esquerda e zero-dimensional tal que $\varphi^{\sim}$ é o filtro das vizinhanças de e. Se $G$ não possui subgrupos finitos não-triviais, então esta topologia é Hausdorff.

Definição 3.44. (a) Fixe uma família $\left\{U_{y}: y \in C\right\}$ de subconjuntos dois-a-dois disjuntos de $G$ tais que $U_{y} \in y$ para todo $y \in C$.

(b) Para cada $y \in C$, defina $A_{y}=\left\{a \in G: a \cdot z \in \overline{U_{y \cdot z}}\right.$ para todo $\left.z \in C\right\}$

Note que $A_{y}=\bigcap_{z \in C}\left\{a \in G: a^{-1} U_{y \cdot z} \in z\right\}$ e que $e \in A_{u}$.

Lema 3.45. Para todo $y \in C, A_{y} \in y$, e se $y, w \in C$ são distintos, então $A_{y} \cap A_{w}=\varnothing$.

Demonstração. Para cada $z \in C, U_{y \cdot z} \in y \cdot z$, ou seja, $\left\{a \in G: a^{-1} U_{y \cdot z} \in z\right\} \in y$. Logo $\bigcap_{z \in C}\left\{a \in G: a^{-1} U_{y \cdot z} \in z\right\}=A_{y} \in y$.

Por contraposição, suponha $y, w \in C$ e $a \in A_{y} \cap A_{w}$. Vale que $a \cdot u \in \overline{U_{y \cdot u}}$ e $a \cdot u \in \overline{U_{w \cdot u}}$, ou seja, $U_{y}, U_{w} \in a \cdot u, \log \circ U_{y} \cap U_{w} \neq \varnothing$, e portanto $y=w$.

Definição 3.46. (a) $X:=\bigcup_{y \in C} A_{y}$. 
(b) Definimos $f: X \rightarrow C$ por $f(a)=y$ se $a \in A_{y}$. Pelo Lema acima isto está bem-definido.

(c) Para cada $z \in C$ e cada $a \in X, V_{z}(a):=\left\{b \in A_{z}: a \cdot b \in A_{f(a) \cdot z}\right\}$.

(d) Para cada $a \in X, V(a):=\bigcup_{z \in C} V_{z}(a)$.

Note que, para cada $a \in X, V(a) \subset X$ e $a \cdot V(a) \subset X$. Além disso, $f(e)=u$, logo para todo $z \in C, V_{z}(e)=A_{z}, \log \mathrm{V}(e)=X$.

Lema 3.47. Para cada $a \in X$ e cada $b \in V(a), f(a \cdot b)=f(a) \cdot f(b)$.

Demonstração. Sejam $a \in X$ e $b \in V(a)$. Tome $z \in C$ tal que $b \in V_{z}(a)$. Como $b \in A_{z}, f(b)=z$, $\operatorname{logo} a \cdot b \in A_{f(a) \cdot z}=A_{f(a) \cdot f(b)}$, de forma que $f(a \cdot b)=f(a) \cdot f(b)$.

Lema 3.48. Para todo $a \in X, V(a) \in \varphi^{\sim}$.

Demonstração. Começaremos mostrando que

(i) dados $y \in C$ e $a \in G, a \in A_{y}$ se e somente se $a \cdot u \in \overline{A_{y}}$, e

(ii) para todos $y, z \in C$, e todo $a \in A_{y}, a \cdot z \in \overline{A_{y \cdot z}}$.

Provando (i), $(\Rightarrow)$ : por contraposição, suponha que $a \cdot u \notin \overline{A_{y}}$. Temos que $a^{-1} A_{y} \notin u$, $\operatorname{logo}$ $G \backslash a^{-1} A_{y}=\left\{b \in G: a \cdot b \notin A_{y}\right\} \in u$. Vale que $a \cdot b \notin A_{y} \Rightarrow a \cdot b \cdot z \notin \overline{U_{y \cdot z}}$ para algum $z \in C$. Como $C$ é finito, tome $z \in C$ tal que $\left\{b \in G: a \cdot b \cdot z \notin \overline{U_{y \cdot z}}\right\} \in u$. Segue que $a \cdot u \cdot z \notin \overline{U_{y \cdot z}}$; como $a \cdot u \cdot z=a \cdot z$, temos que $a \notin A_{y}$.

(i), $(\Leftarrow)$ : Suponha $a \cdot u \in \overline{A_{y}}$. Temos que $a^{-1} A_{y}=\left\{b \in G: a \cdot b \in A_{y}\right\} \in u$, logo para todo $z \in C$, $\left\{b \in G: a \cdot b \cdot z \in \overline{U_{y \cdot z}}\right\} \in u$; logo para todo $z \in C, a \cdot u \cdot z=a \cdot z \in \overline{U_{y \cdot z}}$; ou seja, $a \in A_{y}$.

Provando (ii): $a \cdot z \in \overline{A_{y \cdot z}} \Leftrightarrow a^{-1} A_{y \cdot z} \in z$. Por contraposição, suponha que $a^{-1} A_{y \cdot z} \notin z$; temos que $G \backslash a^{-1} A_{y \cdot z}=\bigcup_{w \in C}\left\{b \in G: a \cdot b \cdot w \notin \overline{U_{y \cdot z \cdot w}}\right\} \in z$, logo tome $w \in C$ tal que $\{b \in G: a \cdot b \cdot w \notin$ $\left.\overline{U_{y \cdot z \cdot w}}\right\} \in z$. Por continuidade, segue que $a \cdot z \cdot w \notin \overline{U_{y \cdot z \cdot w}}$, e portanto $a \notin A_{y}$.

Tendo (i) e (ii), seja $a \in X$. Suponha $V(a) \notin \varphi^{\sim}$ e tome $x \in C^{\sim} \backslash \overline{V(a)}$. Considere $y:=f(a)$ e $z:=$ $x \cdot u$. Como $x \in C^{\sim}, z \in C$, $\operatorname{logo} x \notin \overline{V_{z}(a)}$ e assim $G \backslash V_{z}(a)=\left\{b \in G: b \notin A_{z}\right.$ ou $\left.a \cdot b \notin A_{y \cdot z}\right\} \in x$. Usando (i), temos que $\left\{b \in G: b \notin A_{z}\right.$ ou $\left.a \cdot b \notin A_{y \cdot z}\right\}=\left\{b \in G: b \cdot u \notin \overline{A_{z}}\right.$ ou $\left.a \cdot b \cdot u \notin \overline{A_{y \cdot z}}\right\}$, $\operatorname{logo}\left\{b \in G: b \cdot u \notin \overline{A_{z}}\right\} \in x$, ou $\left\{a \cdot b \cdot u \notin \overline{A_{y \cdot z}}\right\} \in x$. Isto é, $x \cdot u \notin \overline{A_{z}}$ ou $a \cdot x \cdot u \notin \overline{A_{y \cdot z}}$. Como $x \cdot u=z$, temos: $z \notin \overline{A_{z}}$ ou $a \cdot z \notin \overline{A_{y \cdot z}}$. A primeira opção contraria o Lema 3.45 , e a segunda contraria o item (ii), pois $a \in A_{f(a)}=A_{y}$.

Lema 3.49. $X$ é aberto na topologia invariante à esquerda definida em $G$ ao se tomar $\varphi^{\sim}$ como base de vizinhanças de e.

Demonstração. Dado $a \in X$, vimos que $V(a) \in \varphi^{\sim}$ no Lema anterior, logo $a \cdot V(a)$ é aberto; e além disso, $a \in a \cdot V(a) \subset X$. 
Lema 3.50. Dado qualquer $\varnothing \neq Y \subset X$ aberto na topologia definida por $\varphi^{\sim}$, vale que $f[Y]=C$.

Demonstração. Primeiramente, dado $U \in \varphi^{\sim}$, temos que $C \subset C^{\sim} \subset \bar{U}$, ou seja, para todo $y \in C$, $U \in y$. Como $A_{y} \in y$, temos que $U \cap A_{y} \neq \varnothing$ para todo $y \in C$; portanto, $f[U]=C$ pela definição de $f$. Agora, seja $a \in Y$. Tome $U \in \varphi^{\sim}$ tal que $a \cdot U \subset Y$, e $U \subset V(a)$. Pelo Lema 3.47, temos que $f[a \cdot U]=f(a) \cdot f[U]$. Assim, temos que $C=f(a) \cdot C=f(a) \cdot f[U]=f[a \cdot U] \subset f[Y]$. Como $f: X \rightarrow C$, então $f[Y]=C$.

Teorema 3.51. Se G é um grupo enumerável que não possui subgrupos finitos não-triviais, então $G^{*}$ não possui grupo finitos não-triviais.

Demonstração. Suporemos que $C \subset G^{*}$ é um subgrupo finito tal que $C \cong \mathbb{Z}_{p}$ para algum $p>1$ e disso obteremos uma contradição. Como todo grupo finito não-trivial possui um subgrupo cíclico, seguirá o resultado em geral.

Tome então $\gamma: C \rightarrow \mathbb{Z}_{p}$ isomorfismo e para cada $i \in \mathbb{Z}_{p}$ defina $y_{i}=\gamma^{-1}(i)$. Considere $h:=\gamma \circ f$. Temos que $h: X \rightarrow \mathbb{Z}_{p}$ e $h(a)=i$ se e somente se $f(a)=y_{i}$.

Assuma que $G$ possui a topologia produzida no Lema 3.43. Então pelos Lemas 3.43,3.47, 3.48, 3.49 e 3.50, as hipóteses do Lema 3.38 estão satisfeitas, exceto: $X$ não possui pontos isolados na topologia relativa. Para ver isso, note que o 3.50 implica que todo aberto de $X$ tem pelo menos $p$ elementos. Como $p>1, X$ não possui pontos isolados. Dessa forma, podemos aplicar o Lema 3.38 e assumir que $x(t)$ e $X(t)$ foram escolhidos para cada $t \in F$ satisfazendo as condições (1) - (13).

Temos que $A_{y_{\overline{1}}} \in y_{\overline{1}}$, e se $a \in A_{\bar{y}_{\overline{1}}}, f(a)=y_{\overline{1}}$, logo $h(a)=\overline{1}$. Se $a=x(t)$, pela condição (8), $h(a)=g_{p}(t)$; portanto, como $x[F]=X \supset A_{y_{\overline{1}}}, A_{y_{\overline{1}}} \subset\left\{x(t): t \in F\right.$ e $\left.g_{p}(t)=\overline{1}\right\}$. Como $g_{p}(t)=\overline{1} \Leftrightarrow c(t) \equiv 1(\bmod p)$, segue que $\{x(t): t \in F$ e $c(t) \equiv 1(\bmod p)\} \in y_{\overline{1}}$.

Agora, note que $c(t) \equiv 1(\bmod p) \Rightarrow$ existe $m \in \omega$ tal que $c(t)=m p+1$. Dividindo $m$ por $p$, existem $q, r \in \omega, r<p$, tais que $m=q p+r$, de forma que $c(t)=q p^{2}+r p+1$, e logo $c(t) \equiv r p+1$ $\left(\bmod p^{2}\right)$. Por outro lado, se $c(t) \equiv r p+1\left(\bmod p^{2}\right)$, então existe $q \in \omega$ tal que $c(t)=q p^{2}+r p+1$, e $\log o c(t) \equiv 1(\bmod p)$. Defina então, para cada $0 \leq r<p, B_{r}=\{x(t): t \in F$ e $c(t) \equiv r p+1$ $\left.\left(\bmod p^{2}\right)\right\}$. A condição $(9)$ do Lema 3.38 nos garante que se $x(s)=x(t)$ então $c(s)=c(t), \operatorname{logo}$ $B_{r} \cap B_{k}=\varnothing$ para $r, k$ distintos. Como $A_{y_{\overline{1}}} \subset\{x(t): t \in F$ e $c(t) \equiv 1(\bmod p)\}=\bigcup_{r=0}^{p-1} B_{r}$, então podemos tomar o único $r<p$ tal que $B_{r} \in y_{\overline{1}}$.

Seja $n \in \mathbb{N}$. Vale que $X\left(u_{n}\right)$ é aberto e $e=x\left(u_{n}\right) \in X\left(u_{n}\right)$, logo $X\left(u_{n}\right)$ é uma vizinhança de $e$, de modo que $X\left(u_{n}\right) \in \varphi^{\sim}=\bigcap C^{\sim} \subset \bigcap C \subset y_{\overline{1}}$. Dado $t \in F$, vale que $\min \operatorname{supp}(t)>n$ se e só se $t \in u_{n} \frown F \backslash\left\{u_{m}: m \in \mathbb{N}\right\}$. Como, pela condição (12) do Lema 3.38, $x\left[u_{n} \frown F\right]=X\left(u_{n}\right)$, segue que $\{x(t): t \in F$ e $\min \operatorname{supp}(t)>n\}=X\left(u_{n}\right) \backslash\{e\} \in y_{\overline{1}}$.

Para cada $n \in \mathbb{N}$, considere $E_{n}=\left\{x\left(s_{1}+\cdots+s_{n}\right)\right.$ : para cada $1 \leq i \leq n, s_{i} \in F, c\left(s_{i}\right) \equiv r p+1$ $\left(\bmod p^{2}\right)$, e se $\left.i<n, s_{i}<<s_{i+1}\right\}$. Mostraremos por indução que para cada $n \in \mathbb{N}, E_{n} \in y_{\overline{1}}^{n}$. 
Como $E_{1}=B_{r} \in y_{\overline{1}}$, vale para $n=1$. Suponha então que $E_{n} \in y_{\overline{1}}^{n}$. Verificaremos que $E_{n} \subset$ $\left\{a \in G: a^{-1} E_{n+1} \in y_{\overline{1}}\right\}$, e consequentemente $E_{n+1} \in y_{\overline{1}}{ }^{n} \cdot y_{\overline{1}}=y_{\overline{1}}{ }^{n+1}$. Logo seja $a \in E_{n}$ e tome $s_{1}<<\ldots<<s_{n}$ em $F$ tais que para cada $i, c\left(s_{i}\right) \equiv r p+1\left(\bmod p^{2}\right)$ e $a=x\left(s_{1}+\cdots+s_{n}\right)$. Considere $D=\left\{x(t): t \in F\right.$ e $\left.\min \operatorname{supp}(t)>\max \operatorname{supp}\left(s_{n}\right)+1\right\}$. Pelo observado acima, $B_{r} \cap D \in y_{\overline{1}}$, $\operatorname{logo}$ basta ver que $B_{r} \cap D \subset a^{-1} E_{n+1}$. Seja então $t \in F$ tal que $\min \operatorname{supp}(t)>\max \operatorname{supp}\left(s_{n}\right)+1 \mathrm{e}$ $c(t) \equiv r p+1\left(\bmod p^{2}\right)$. Pela condição $(10)$ do Lema 3.38 , temos que $x\left(s_{1}+\cdots+s_{n}\right) \cdot x(t)=$ $x\left(s_{1}+\cdots+s_{n}+t\right) \in E_{n+1}, \operatorname{logo} a \cdot x(t) \in E_{n+1}$ como desejado.

Agora, com a indução completa, temos que: $y_{\overline{1}}^{p+1}=y_{\overline{1}}$, $\operatorname{logo} E_{p+1} \in y_{\overline{1}}$, logo tome $a \in E_{p+1} \cap B_{r}$. Sejam então $s_{1}<<\ldots<<s_{p+1}$ em $F$ tais que $c\left(s_{i}\right) \equiv r p+1\left(\bmod p^{2}\right)$ e $a=x\left(s_{1}+\cdots+s_{p+1}\right)$. Temos então o seguinte, pela condição (10) do Lema 3.38: $c\left(s_{1}+\cdots+s_{p+1}\right)=c\left(s_{1}\right)+\cdots c\left(s_{p+1}\right) \equiv$ $(p+1)(r p+1)=r p^{2}+p+r p+1 \equiv(r+1) p+1\left(\bmod p^{2}\right)$. Ou seja, $a \in B_{r+1}$, e portanto $B_{r} \cap B_{r+1} \neq \varnothing\left(\right.$ caso $\left.r=p-1, B_{p-1} \cap B_{0} \neq \varnothing\right)$. $\underline{\text { Contradição. }}$ 


\section{Apêndice A}

\section{Grupos Abelianos Divisíveis}

O material encontrado aqui foi retirado do livro "Abelian Groups", de László Fuchs ([10]). Ele é utilizado na seção 3.2 do capítulo 3, e por isso é apresentado de forma resumida, assumindo um conhecimento mínimo sobre grupos abelianos.

Definição A.1. Seja $(G,+)$ um grupo abeliano. Denote por $e$ seu elemento neutro, e $-g$ o oposto de $g \in G$. Como é usual, denotamos para cada $n \in \mathbb{Z}$ e cada $g \in G$ o elemento $n g \in G$ definido recursivamente por $0 g=e,(n+1) g=n g+g$ para $n \in \mathbb{N}$ e $n g=-((-n) g)$ para cada $n \in \mathbb{Z}$ negativo; de forma que para todos $n, m \in \mathbb{Z}$, e $g, h \in G,(n+m) g=n g+m g,(n m) g=n(m g)$ e $n(g+h)=n g+n h$. Denotamos a ordem de um elemento $g$ por $O(g)$, caso seja finita.

Fixada tal notação, diz-se que $G$ é um grupo divisível se para todo $n \in \mathbb{N}$ e todo $a \in G$ existe $x \in G$ tal que $n x=a$. Equivalentemente, se para todo $n \in \mathbb{N}$ vale que $n G=G$.

Lembramos que dado um primo $p, G$ é um $p$-grupo se a ordem de todo elemento é uma potência de $p$.

Lema A.2. Seja $G$ um grupo abeliano.

(a) Se para todo p primo, $p G=G$, então $G$ é divisivel.

(b) Se $G$ é um p-grupo, então $G$ é divisivel se e somente se $p G=G$.

(c) Uma soma direta $\sum_{\lambda \in \Lambda} G_{\lambda}$ é divisivel se e somente se cada $G_{\lambda}$ for divisivel.

(d) Se $f: G \rightarrow H$ é um homomorfismo sobrejetor, e $G$ é divisivel, então $H$ é divisivel.

Demonstração. (a): Seja $n \in \mathbb{N}$. Pelo Teorema Fundamental da Aritmética, $n=p_{1} \ldots p_{k}$ para certos primos $p_{1}, \ldots, p_{k}$ (com repetição se necessário). Um argumento indutivo em $n$ mostra que $n G=\left(p_{1} \ldots p_{k-1}\right)\left(p_{k} G\right)=\left(p_{1} \ldots p_{k-1}\right) G=G$.

(b): O "somente se" é óbvio. Suponha $p G=G$. Devido a (a), basta ver que $q G=G$ para todos $q$ primos distintos de $p$. Sejam $q \neq p$ primo e $a \in G$. Seja $n \in \mathbb{N}$ tal que $p^{n} a=e$. Como 
$\operatorname{mdc}\left(p^{n}, q\right)=1$, tome $s, t \in \mathbb{Z}$ tais que $s p^{n}+t q=1$. Temos então: $a=1 a=\left(s p^{n}+t q\right) a=$ $s p^{n} a+t q a=s e+t q a=e+t q a=t q a=q(t a)$, de forma que $a \in q G$.

(c): Se $\sum_{\lambda \in \Lambda} G_{\lambda}$ é divisível, dados $n \in \mathbb{N}, \mu \in \Lambda$ e $a \in G_{\mu} \backslash\{e\}$, como $a \in \sum_{\lambda \in \Lambda} G_{\lambda}$, então existe $x \in \sum_{\lambda \in \Lambda} G_{\lambda}$ tal que $n x=a$. Tome $\lambda_{1}, \ldots, \lambda_{r} \in \Lambda$ tais que $x=x_{\lambda_{1}}+\cdots+x_{\lambda_{r}} \operatorname{com} x_{\lambda_{j}} \in G_{\lambda_{j}} \backslash\{e\}$. Temos que $n x_{\lambda_{1}}+\cdots+n x_{\lambda_{r}}=a$. Portanto $r=1, \lambda_{1}=\mu$ e assim $n x_{\mu}=a \operatorname{com} x_{\mu} \in G_{\mu}$.

Por outro lado se cada $G_{\lambda}$ é divisível, dados $\lambda_{1}, \ldots, \lambda_{r} \in \Lambda$ e $a_{\lambda_{j}} \in G_{\lambda_{j}}$, e $n \in \mathbb{N}$, existem $x_{\lambda_{j}} \in G_{\lambda_{j}}$ tais que $n x_{\lambda_{j}}=a_{\lambda_{j}}$, e assim $n\left(x_{\lambda_{1}}+\cdots+x_{\lambda_{r}}\right)=a_{\lambda_{1}}+\cdots a_{\lambda_{r}}$ (note que não usamos neste caso que a soma é direta).

(d): Sejam $n \in \mathbb{N}$ e $h \in H$. Tome $g \in G$ tal que $f(g)=h$, e tome $x \in G$ tal que $n x=g$. Temos então que $h=f(g)=f(n x)=n f(x)$.

Agora que sabemos que somas diretas e quocientes de grupos divisíveis são divisíveis, e usando o evidente fato de que $(\mathbb{Q},+)$ é divisível, temos o seguinte resultado:

Teorema A.3. Todo grupo abeliano $G$ pode ser imerso em um grupo divisivel.

Demonstração. Todo grupo abeliano $G$ é a imagem homomorfa de um grupo abeliano livre $F$. Logo, para algum $N<F, G \cong F / N$. Note agora que, sendo $\left\{a_{\lambda}: \lambda \in \Lambda\right\}$ o alfabeto de $F, F=\sum_{\lambda \in \Lambda}\left\langle a_{\lambda}\right\rangle$, em que $\left\langle a_{\lambda}\right\rangle$ é o grupo abeliano livre sobre um gerador - e portanto isomorfo a $\mathbb{Z}$. $\mathbb{Z}$ está imerso em $\mathbb{Q}$, que é divisível. Logo $F$ está imerso na soma direta externa de $\Lambda$ cópias de $\mathbb{Q}$ - chamemo-la de $D$.

Segue que $G \cong F / N<D / N$, e $D / N$ é divisível.

Teorema A.4. Sejam $H$ subgrupo de $G$ grupo abeliano e $D$ um grupo divisivel. Então todo homomorfismo $h: H \rightarrow D$ possui uma extensão $\widetilde{h}: G \rightarrow D$.

Demonstração. Aplicaremos o Lema de Zorn. Dados $H<U, U^{\prime}<G$ e $f: U \rightarrow D, f^{\prime}: U^{\prime} \rightarrow D$ extensões de $h$, definimos $(U, f) \leq\left(U^{\prime}, f^{\prime}\right)$ se e só se $U \subset U^{\prime}$ e $f^{\prime}$ for uma extensão de $f$. Dada $\mathcal{C}$ cadeia, defina $V=\bigcup_{(U, f) \in \mathcal{C}} U$ e $F=\bigcup_{(U, f) \in \mathcal{C}} f$; vale que é um limitante superior para $\mathcal{C}$. Logo podemos aplicar o Lema de Zorn e tomar $(V, F)$ maximal. Provaremos então que $V=G$.

Suponha que $g \in G \backslash V$. Caso não exista $n \in \mathbb{N}$ tal que $n g \in V$, simplesmente tome $x \in V$ qualquer e defina $F^{\prime}:\langle V, g\rangle \rightarrow D$ por $F^{\prime}(a+t g)=F(a)+t x$ para todo $a \in V$ e $t \in \mathbb{Z}$; temos que $F^{\prime}$ é um homomorfismo.

Caso exista $n \in \mathbb{N}$ tal qie $n g \in V$, tome $n$ mínimo com tal propriedade. Tome $x \in D$ tal que $n x=F(n g)$. Defina então $F^{\prime}:\langle V, g\rangle \rightarrow D$ por $F^{\prime}(a+t g)=F(a)+t x$ para todo $0 \leq t<n$. Segue que $F^{\prime}$ é um homomorfismo.

Em ambos os casos, teríamos que $(V, F)$ não seria maximal, uma contradição. Portanto, $V=G$ e $\operatorname{definimos} \widetilde{h}=F$. 
Definição A.5. Seja $G$ um grupo abeliano. Um sistema de equações (lineares) em $G$ é um conjunto de equações do tipo $n_{\nu, 1} x_{\lambda_{\nu, 1}}+\cdots+n_{\nu, k_{\nu}} x_{\lambda_{\nu, k_{\nu}}}=a_{\nu}$, em que $\nu \in \mathrm{N}$ conjunto indexador qualquer, $k_{\nu} \in \mathbb{N}, a_{\nu} \in G, n_{\nu, i} \in \mathbb{N}$, e $x_{\lambda_{\nu, i}} \in\left\{x_{\lambda}: \lambda \in \Lambda\right\}$, um conjunto de incógnitas.

Uma condição conhecidamente necessária para que o sistema de equações tenha solução (esta noção será formalizada abaixo) é que, sempre que uma combinação linear dos lados esquerdos de equações resultar em 0 , então a combinação linear correspondente dos lados direitos também resulta em 0 - isto é, na identidade de $G$. Mais formalmente, considere $X$ o grupo abeliano livre sobre o alfabeto $\left\{x_{\lambda}: \lambda \in \Lambda\right\}$ e $Y$ o subgrupo de $X$ gerado pelos elementos $l_{\nu}(x)=n_{\nu, 1} x_{\lambda_{\nu, 1}}+\cdots+$ $n_{\nu, k_{\nu}} x_{\lambda_{\nu, k_{\nu}}}, \nu \in \mathrm{N}$. A condição de que uma combinação linear dos lados esquerdos de equações que resulta em 0 corresponde a uma combinação linear dos lados direitos que resulta na identidade de $G$ é apenas uma forma (equivalente) de dizer que a função $l_{\nu}(x) \mapsto a_{\nu}$ induz um homomorfismo $f: Y \rightarrow G$. Isso nos leva a definir:

Definição A.6. Seja $G$ um grupo abeliano e $n_{\nu, 1} x_{\lambda_{\nu, 1}}+\cdots+n_{\nu, k_{\nu}} x_{\lambda_{\nu, k_{\nu}}}=a_{\nu}$, em que $\nu \in \mathrm{N}$, $k_{\nu} \in \mathbb{N}, a_{\nu} \in G, n_{\nu, i} \in \mathbb{N}$, e $x_{\lambda_{\nu, i}} \in\left\{x_{\lambda}: \lambda \in \Lambda\right\}$, um sistema de equações. Denotando $l_{\nu}(x)=$ $n_{\nu, 1} x_{\lambda_{\nu, 1}}+\cdots+n_{\nu, k_{\nu}} x_{\lambda_{\nu, k_{\nu}}}$ para cada $\nu \in \mathrm{N}$, dizemos que o sistema é compatível se a função $l_{\nu}(x) \mapsto a_{\nu}$ induz um homomorfismo $f: Y \rightarrow G$, em que $Y$ é o subgrupo, do grupo livre sobre $\left\{x_{\lambda}: \lambda \in \Lambda\right\}$, gerado por $\left\{l_{\nu}(x): \nu \in \mathrm{N}\right\}$.

Definição A.7. Seja $G$ um grupo abeliano e $n_{\nu, 1} x_{\lambda_{\nu, 1}}+\cdots+n_{\nu, k_{\nu}} x_{\lambda_{\nu, k_{\nu}}}=a_{\nu}$, em que $\nu \in \mathrm{N}$, $k_{\nu} \in \mathbb{N}, a_{\nu} \in G, n_{\nu, i} \in \mathbb{N}$, e $x_{\lambda_{\nu, i}} \in\left\{x_{\lambda}: \lambda \in \Lambda\right\}$, um sistema de equações. Uma solução para o sistema de equações é uma família indexada $\left\{g_{\lambda}: \lambda \in \Lambda\right\} \subset G$ que torna as equações verdadeiras ou seja, tal que para cada $\nu \in \mathrm{N}, n_{\nu, 1} g_{\lambda_{\nu, 1}}+\cdots+n_{\nu, k_{\nu}} g_{\lambda_{\nu, k_{\nu}}}=a_{\nu}$.

Note que uma solução $\left\{g_{\lambda}: \lambda \in \Lambda\right\}$ induz a função $x_{\lambda} \mapsto g_{\lambda}$, e esta induz a um homomorfismo $g$ do grupo livre $X$ em $G$. Portanto, como anunciado acima, se o sistema de equações possui solução $\left\{g_{\lambda}: \lambda \in \Lambda\right\}$, claramente ele é compatível, pois a função $l_{\nu}(x) \mapsto a_{\nu}$ é uma restrição de $g$, do que $\left.g\right|_{Y}$ é um homomorfismo. Por outro lado, dado um sistema compatível de equações e $f: Y \rightarrow G$ seu homomorfismo induzido, uma extensão $f$ a $X, g: X \rightarrow G$, induziria uma solução do sistema definindo $g_{\lambda}=g\left(x_{\lambda}\right)$ para cada $\lambda \in \Lambda$. Além do que, diferentes extensões de $f$ induzem diferentes soluções (pois diferentes homomorfismos sobre $X$ não podem assumir os mesmos valores sobre todos os $\left.x_{\lambda}\right)$. Temos então:

Lema A.8. Seja $G$ um grupo abeliano e $n_{\nu, 1} x_{\lambda_{\nu, 1}}+\cdots+n_{\nu, k_{\nu}} x_{\lambda_{\nu, k_{\nu}}}=a_{\nu}$, em que $\nu \in \mathrm{N}, k_{\nu} \in \mathbb{N}$, $a_{\nu} \in G, n_{\nu, i} \in \mathbb{N}$, e $x_{\lambda_{\nu, i}} \in\left\{x_{\lambda}: \lambda \in \Lambda\right\}$, um sistema de equações compativel. Seja $f: Y \rightarrow G$ seu homomorfismo induzido. O sistema é solúvel (ou seja, possui solução) se e somente se existe $g: X \rightarrow G$ extensão de $f$, caso em que $\left\{g\left(x_{\lambda}\right): \lambda \in \Lambda\right\}$ é solução. Além disso, diferentes extensões de $f$ correspondem a diferentes soluções. 
Com estas observações, podemos enunciar e provar:

Teorema A.9. Todo sistema de equações compativel sobre um grupo divisivel D possui solução em $D$.

Demonstração. Um sistema de equações compatível induz o homomorfismo $f: Y \rightarrow D$ (de acordo com as notações acima). Pelo Teorema A.4, $f$ admite uma extensão $g: X \rightarrow D$. Pelo Lema acima, isso implica que o sistema possui uma solução.

Veremos agora que todo subgrupo divisível é um somando direto de qualquer grupo em que esteja imerso.

Teorema A.10. Seja $G$ grupo abeliano, e seja $D$ um subgrupo divisível. Então existe $B$ subgrupo de $G$ tal que $D \oplus B=G$.

Demonstração. Tome $\left\{g_{\lambda}: \lambda \in \Lambda\right\}$ tal que $G=\left\langle D,\left\{g_{\lambda}\right\}_{\lambda \in \Lambda}\right\rangle\left(\left\{g_{\lambda}\right\}_{\lambda \in \Lambda}\right.$ pode ser pensado como uma família de representantes de um conjunto gerador de $G / D)$. Considere todas as combinações lineares de elementos de $\left\{g_{\lambda}: \lambda \in \Lambda\right\}$ que resultam em elementos de $D$, e as liste da seguinte forma: $n_{\nu, 1} g_{\lambda_{\nu, 1}}+\cdots+n_{\nu, k_{\nu}} g_{\lambda_{\nu, k_{\nu}}}=a_{\nu}$, em que $\nu \in \mathrm{N}, k_{\nu} \in \mathbb{N}, a_{\nu} \in D, n_{\nu, i} \in \mathbb{N}$, e $g_{\lambda_{\nu, i}} \in\left\{g_{\lambda}: \lambda \in \Lambda\right\}$. Afirmamos então que o sistema de equações $n_{\nu, 1} x_{\lambda_{\nu, 1}}+\cdots+n_{\nu, k_{\nu}} x_{\lambda_{\nu, k_{\nu}}}=a_{\nu}$, em que $\nu \in \mathrm{N}, k_{\nu} \in \mathbb{N}$, $a_{\nu} \in G, n_{\nu, i} \in \mathbb{N}$, e $x_{\lambda_{\nu, i}} \in\left\{x_{\lambda}: \lambda \in \Lambda\right\}$ é compatível. Como antes, denote, para cada $\nu \in \mathrm{N}$, $l_{\nu}(x)=n_{\nu, 1} x_{\lambda_{\nu, 1}}+\cdots+n_{\nu, k_{\nu}} x_{\lambda_{\nu, k_{\nu}}}$, e similarmente denote $l_{\nu}(g)=n_{\nu, 1} g_{\lambda_{\nu, 1}}+\cdots+n_{\nu, k_{\nu}} g_{\lambda_{\nu, k_{\nu}}}$. A função $x_{\lambda} \mapsto g_{\lambda}, \lambda \in \Lambda$, determina um homomorfismo $f: X \rightarrow G$ ( $X$ é o grupo livre sobre $\left.\left\{x_{\lambda}: \lambda \in \Lambda\right\}\right)$. Dessa forma, para cada $\nu \in \mathrm{N}, f\left(l_{\nu}(x)\right)=l_{\nu}(g)$. Portanto, se para algum $\mathrm{N}^{\prime} \subset \mathrm{N}$ finito, $\sum_{\nu \in \mathrm{N}^{\prime}} l_{\nu}(x)=e_{X}$, então $e_{G}=f\left(e_{X}\right)=f\left(\sum_{\nu \in \mathrm{N}^{\prime}} l_{\nu}(x)\right)=\sum_{\nu \in \mathrm{N}^{\prime}} f\left(l_{\nu}(x)\right)=\sum_{\nu \in \mathrm{N}^{\prime}} l_{\nu}(g)=$ $\sum_{\nu \in \mathrm{N}^{\prime}} a_{\nu}$; de forma que o sistema é de fato compatível. Assim, pelo Teorema A.9 acima, o sistema admite uma solução em $D$ - denotemo-la por $\left\{h_{\lambda}: \lambda \in \Lambda\right\}$. Defina $B=\left\langle\left\{g_{\lambda}-h_{\lambda}: \lambda \in \Lambda\right\}\right\rangle$. Vale que $G=\langle D, B\rangle$. Para ver que $G=D \oplus B$, resta ver que $D \cap B=\left\{e_{G}\right\}$. Suponha então $\Lambda^{\prime} \subset \Lambda$ finito e $\left\{s_{\lambda}: \lambda \in \Lambda^{\prime}\right\} \subset \mathbb{Z}$ tais que $\sum_{\lambda \in \Lambda^{\prime}} s_{\lambda}\left(g_{\lambda}-h_{\lambda}\right) \in D$. Temos então que $\sum_{\lambda \in \Lambda^{\prime}} s_{\lambda} g_{\lambda}=: a \in D$, de forma que $\sum_{s_{\lambda} \in \Lambda^{\prime}} s_{\lambda} x_{\lambda}=a$ é uma das equações do sistema acima, e portanto vale que $\sum_{\lambda \in \Lambda^{\prime}} s_{\lambda} h_{\lambda}=a$. Segue que $\sum_{\lambda \in \Lambda^{\prime}} s_{\lambda}\left(g_{\lambda}-h_{\lambda}\right)=e_{G}$, como queríamos.

Como foi usado acima, $\mathbb{Q}$ é um grupo divisível, assim como, por exemplo, $\mathbb{R}$ e $\mathbb{C}$. $\mathbb{Z}$, por exemplo, é um claro exemplo de grupo não-divisível. Uma classe de grupos divisíveis que nos será relevante são os grupos quasicíclicos. Existem algumas formas de apresentar esse grupo, a usada neste texto será a seguinte: dado $p$ primo, o grupo $p$-quasicíclico é $Z\left(p^{\infty}\right)=\left(\mathbb{Z}\left[\frac{1}{p}\right]\right) / \mathbb{Z}$ com a operação de adição no quociente, sendo que $\mathbb{Z}\left[\frac{1}{p}\right]$ é o anel dos racionais $p$-ádicos, ou seja, 
$\left\{\frac{m}{p^{n}}: m \in \mathbb{Z}\right.$ e $\left.n \in \mathbb{N}\right\}$. Costumeiramente, iremos denotar os elementos de $Z\left(p^{\infty}\right)$ pelos seus representantes em $[0,1)$, lembrando que o quociente é um "descarte" da parte inteira - por exemplo, temos que $\frac{p-1}{p}+\frac{p-1}{p}=\frac{2 p-2}{p}=\frac{p}{p}+\frac{p-2}{p}=\frac{p-2}{p}$.

Claramente temos as seguintes relações: $p\left(\frac{1}{p}\right)=1$ e $p\left(\frac{1}{p^{n}}\right)=\frac{1}{p^{n-1}}$ para todo $n>1$. De fato, estas relações descrevem o grupo, isto é, o grupo $p$-quasicíclico possui uma descrição por geradores e relações $Z\left(p^{\infty}\right)=\left\langle g_{1}, g_{2}, g_{3}, \ldots \mid p g_{1}=e, p g_{2}=g_{1}, p g_{3}=g_{2}, \ldots\right\rangle$. Com esta descrição, qualquer elemento de $Z\left(p^{\infty}\right) \backslash\{e\}$ é da forma $m g_{n} \operatorname{com} m<p^{n}$.

O grupo $p$-quasicíclico é um $p$-grupo, logo usaremos o Lema A.2(b) para ver que é divisível. Seja $\frac{m}{p^{n}} \in Z\left(p^{\infty}\right)$. Vale que $p\left(\frac{m}{p^{n+1}}\right)=\frac{m}{p^{n}}$, e portanto $Z\left(p^{\infty}\right)$ é divisível.

Pelo Lema A.2(c), qualquer soma direta de racionais e grupos quasicíclicos é divisível. De fato, o teorema que será provado aqui diz que estes são os únicos grupos divisíveis possíveis. Porém, para prová-lo, precisaremos ainda de duas outras noções sobre grupos abelianos: torsão e independência.

Definição A.11. Seja $G$ um grupo abeliano. Dizemos que $g \in G$ é elemento de torsão se sua ordem for finita. Dizemos que $T<G$ é subgrupo de torsão se todos os seus elementos forem de torsão. Dizemos que $F<G$ é livre de torsão se nenhum elemento além de $e$ for de torsão.

Lema A.12. Seja $G$ um grupo abeliano. Então o conjunto $T$ de todos os elementos de torsão de $G$ é um subgrupo, chamado de subgrupo de torsão maximal. Além disso, se $G$ for divisivel, então T também o é.

Demonstração. Dados, $a, b \in T$, sejam $n, m \in \mathbb{N}$ tais que $n a=m b=e$. Então $n m(a-b)=$ $n m a-n m b=e-e=e$. Agora, suponha que $G$ seja divisível, e seja $r \in \mathbb{N}$. Tome $x \in G$ tal que $r x=a$. Então $n r x=n a=e, \operatorname{logo} x \in T$, o que mostra que $T$ é divisível.

Definição A.13. Seja $T$ um grupo de torsão. Então, para cada $p$ primo, definimos $T_{p}=\{g \in G$ : existe $n \in \mathbb{N}$ tal que $\left.O(g)=p^{n}\right\}$ a $p$-componente de $T$.

A razão para o nome p-componente é elucidada pelo próximo lema.

Lema A.14. Seja $T$ um grupo de torsão. Então $T=\bigoplus_{p \text { primo }} T_{p}$.

Demonstração. Obviamente, $T_{p} \cap T_{p^{\prime}}=\{e\}$ se $p \neq p^{\prime}$. E dados $a, b \in T_{p}$, sejam $n, n^{\prime} \in \mathbb{N}$ tais que $p^{n} a=p^{n^{\prime}} b=e$. Sem perda de generalidade, $n^{\prime}<n$, logo $p^{n}(a-b)=p^{n} a-p^{n} b=e-e=e$, de modo que cada $T_{p}$ é de fato um subgrupo. Resta ver que eles geram $T$. Seja $a \in T$ qualquer, e tome $r \in \mathbb{N}$ tal que $r a=e$. Para alguns primos $p_{1}, \ldots, p_{k}$, e alguns $n_{1}, \ldots, n_{k} \in \mathbb{N}, r=p_{1}^{n_{1}} \ldots p_{k}{ }^{n_{k}}$. Defina $r_{i}=\frac{r}{p_{i}{ }^{n_{i}}}$. Note que $\operatorname{mdc}\left(r_{1}, \ldots, r_{k}\right)=1, \operatorname{logo}$ tome $s_{1}, \ldots, s_{k} \in \mathbb{Z}$ tais que $s_{1} r_{1}+\cdots+s_{k} r_{k}=1$. Temos então $a=\left(s_{1} r_{1}+\cdots+s_{k} r_{k}\right) a=s_{1} r_{1} a+\cdots+s_{k} r_{k} a$, em que, para cada $i, p_{i}{ }^{n_{i}} s_{i} r_{i} a=$ $s_{i} r_{i} p_{i}^{n_{i}} a=s_{i} r a=s_{i} e=e$, de forma que $s_{i} r_{i} a \in T_{p_{i}}$. 
Definição A.15. Seja $G$ um grupo abeliano. Dizemos que $L \subset G$ é um conjunto independente se sempre que $a_{1}, \ldots, a_{k} \in L, n_{1}, \ldots, n_{k} \in \mathbb{Z}$ e $n_{1} a_{1}+\cdots+n_{k} a_{k}=e$, então $n_{1} a_{1}=\cdots=n_{k} a_{k}=e$.

Claramente, $L$ é independente se e somente se cada subconjunto finito de $L$ é independente. Assim é fácil ver que a união de qualquer cadeia de conjuntos independentes é também independente. Ou seja, o Lema de Zorn garante que todo grupo não-trivial possui um conjunto independente maximal.

Lema A.16. Seja $T$ um p-grupo. Então dado conjunto independente maximal $\left\{b_{\lambda}: \lambda \in \Lambda\right\}$, pode-se obter um conjunto independente maximal $\left\{a_{\lambda}: \lambda \in \Lambda\right\}$ tal que $O\left(a_{\lambda}\right)=p$ para todo $\lambda \in \Lambda$.

Demonstração. Para cada $\lambda \in \Lambda$, se $O\left(b_{\lambda}\right)=p$, defina $a_{\lambda}=b_{\lambda}$. Caso $O\left(b_{\lambda}\right)=p^{n} \operatorname{com} n>1$, defina $a_{\lambda}=p^{n-1} b_{\lambda}$. Claramente $O\left(a_{\lambda}\right)=p$ para cada $\lambda$. Suponha que $m_{1} a_{\lambda_{1}}+\cdots+m_{k} a_{\lambda_{k}}=e$. Tome $n_{1}, \ldots, n_{k}$ tais que $a_{\lambda_{i}}=p^{n_{i}-1} b_{\lambda_{i}}$. Temos então que $m_{1} p^{n_{1}-1} b_{\lambda_{1}}+\cdots+m_{k} p^{n_{k}-1} b_{\lambda_{k}}=e$, do que segue $m_{i} p^{n_{i}-1} b_{\lambda_{i}}=e$ para cada $i$, ou seja, $m_{i} a_{\lambda_{i}}=e$.

Dessa forma, $\left\{a_{\lambda}: \lambda \in \Lambda\right\}$ é independente. Para ver que é maximal, seja $g \in T$. Como $\left\{b_{\lambda}: \lambda \in\right.$ $\Lambda\} \cup\{g\}$ não é independente, tome $m, m_{1}, \ldots, m_{k} \in \mathbb{Z}$ e $\lambda_{1}, \ldots, \lambda_{k} \in \Lambda$ tais que $m g+m_{1} b_{\lambda_{1}}+$ $\cdots+m_{k} b_{\lambda_{k}}=e$ mas $m g \neq e$ ou $m_{i} b_{\lambda_{i}} \neq e$ para algum $i$. Caso $m g=e$ teríamos uma violação da independência de $\left\{b_{\lambda}: \lambda \in \Lambda\right\}$, logo $m g \neq e$. Como $m g \neq e$, então $I:=\{i \in\{1, \ldots, k\}$ : $\left.m_{i} b_{\lambda_{i}} \neq e\right\} \neq \varnothing$. Para cada $i \in I$, tome $r_{i} \in \mathbb{Z}$ e $s_{i} \in \omega$ tais que $m_{i}=r_{i} p^{s_{i}}$ com $p \nmid r_{i}$. Tome $n_{i}$ tais que $p^{n_{i}-1} b_{\lambda_{i}}=a_{\lambda_{i}}$ para cada $i \in I$ e note que $s_{i} \leq n_{i}-1$. Defina $t_{i}=n_{i}-1-s_{i}$ e defina $J=\left\{j \in I: t_{j}=\max \left\{t_{i}: i \in I\right\}\right\}$. Defina $t=\max \left\{t_{i}: i \in I\right\}$, ou seja, $t=t_{j}$ se e somente se $j \in J$, e $t>t_{i}$ para todo $i \in I \backslash J$. Assim, note que $p^{t} m_{j} b_{\lambda_{j}}=r_{j} a_{\lambda_{j}}$ caso $j \in J$ e $p^{t} m_{i} b_{\lambda_{i}}=e$ caso $i \in\{1, \ldots, k\} \backslash J$. Segue que $e=p^{t}\left(m g+m_{1} b_{\lambda_{1}}+\cdots+m_{k} b_{\lambda_{k}}\right)=p^{t} m g+\sum_{j \in J} r_{j} a_{\lambda_{j}}$. Como $p \nmid r_{j}$, então $r_{j} a_{\lambda_{j}} \neq e$ para todo $j \in J$, o que mostra que $\left\{a_{\lambda}: \lambda \in \Lambda\right\} \cup\{g\}$ não é independente.

Teorema A.17. Todo grupo divisivel é a soma direta de grupos quasicíclicos e de cópias dos racionais.

Demonstração. Seja $D$ um grupo divisível, e seja $T$ seu subgrupo de torsão maximal. Pelo Lema A.12, $T$ é divisível, logo pelo Teorema A.10 $T$ é um somando direto de $D$. Seja $F<D$ tal que $D=T \oplus F$. Como $T \cap F=\{e\}, F$ é livre de torsão; e como é somando direto de $D, F$ também é divisível. Usando o Lema A.14 temos que cada $T_{p}$, para $p$ primo, também é divisível. Mostraremos então que $F$ é uma soma direta de cópias de $\mathbb{Q}$ e que cada $T_{p}$ é uma soma direta de cópias de $Z\left(p^{\infty}\right)$.

Primeiramente, tome $\left\{a_{\lambda}: \lambda \in \Lambda\right\}$ conjunto independente maximal em $T_{p}$. Pelo Lema logo acima, podemos tomá-lo de forma que $O\left(a_{\lambda}\right)=p$ para todo $\lambda \in \Lambda$. Fixe $\lambda$. Pela divisibilidade de $T_{p}$, podemos recursivamente construir $\left(a_{\lambda, n}\right)_{n \in \mathbb{N}}$ de forma que $a_{\lambda, 1}=a_{\lambda}$ e $a_{\lambda, n}=p a_{\lambda, n+1}$ para 
todo $n \in \mathbb{N}$ - o que implica que a sequência é injetora pois estamos em um $p$-grupo. Assim, como $p a_{\lambda}=e, a_{\lambda}$ pertence a um subgrupo $p$-quasicíclico de $T_{p}$, gerado por $\left\{a_{\lambda, n}: n \in \mathbb{N}\right\}$; denotemo-lo $Q_{\lambda}$. Vejamos que $\left\{Q_{\lambda}: \lambda \in \Lambda\right\}$ gera uma soma direta. Seja $\mu \in \Lambda$ e suponha $\lambda_{1}, \ldots, \lambda_{k} \in \Lambda, m_{1}, \ldots, m_{k} \in \mathbb{Z}$, e $n_{1}, \ldots, n_{k} \in \mathbb{N}$ tais que $m_{1} a_{\lambda_{1}, n_{1}}+\cdots+m_{k} a_{\lambda_{k}, n_{k}} \in Q_{\mu} \backslash\{e\}$. Tome $n \in \mathbb{N}$ e $m<p^{n}$ tais que $m_{1} a_{\lambda_{1}, n_{1}}+\cdots+m_{k} a_{\lambda_{k}, n_{k}}=m a_{\mu, n}$. Como $m a_{\mu, n} \neq e$, então $I=\left\{i \in\{1, \ldots, k\}: m_{i} a_{\lambda_{i}, n_{i}} \neq e\right\} \neq \varnothing$. Para cada $i \in I$, tome $r_{i} \in \mathbb{Z}$ e $s_{i} \in \omega$ tais que $m_{i}=r_{i} p^{s_{i}}$ com $p \nmid r_{i}$, e tome $r \in \mathbb{Z}$ e $s \in \omega$ tais que $m=r p^{s} \operatorname{com} p \nmid r$. Defina $t_{i}=n_{i}-1-s_{i}$ e $t^{\prime}=n-1-s$; e defina $J=\left\{j \in I: t_{j}=\max \left(\left\{t_{i}: i \in I\right\} \cup\left\{t^{\prime}\right\}\right)\right\}$. Defina $t=\max \left(\left\{t_{i}: i \in I\right\} \cup\left\{t^{\prime}\right\}\right)$, ou seja, $t=t_{j}$ se e somente se $j \in J$, e $t>t_{i}$ para todo $i \in I \backslash J$. Assim, note que $p^{t} m_{j} a_{\lambda_{j}, n_{j}}=r_{j} a_{\lambda_{j}}$ caso $j \in J$ e $p^{t} m_{i} a_{\lambda_{i}, n_{i}}=e$ caso $i \in\{1, \ldots, k\} \backslash J$. Segue que $p^{t} m a_{\mu, n}=p^{t}\left(m_{1} a_{\lambda_{1}, n_{1}}+\cdots+m_{k} a_{\lambda_{k}, n_{k}}\right)=$ $\sum_{j \in J} r_{j} a_{\lambda_{j}}$. Temos dois casos: se $t^{\prime}<t$, então $p^{t} m a_{\mu, n}=e$; caso $t^{\prime}=t, p^{t} m a_{\mu, n}=r a_{\mu}$; em ambos os casos, temos que a equação acima é uma violação da independência de $\left\{a_{\lambda}: \lambda \in \Lambda\right\}$.

Portanto, temos $Q_{p}:=\bigoplus_{\lambda \in \Lambda} Q_{\lambda}<T_{p}$. Como $Q_{p}$ é divisível, $T_{p}=Q_{p} \oplus S_{p}$; porém $Q_{p}$ contém um conjunto independente maximal, o que implica que $S_{p}=\{e\}$, e assim $Q_{p}=T_{p}$, como queríamos. Agora, vejamos que $F$ é uma soma direta de cópias de $\mathbb{Q}$. Tome $\left\{b_{\lambda}: \lambda \in \Lambda\right\}$ conjunto independente maximal em $F$. Como $F$ é livre de torsão, então para cada $n \in \mathbb{N}$ e cada $\lambda \in \Lambda$ existe um único $x \in F$ tal que $n x=b_{\lambda}$; assim, podemos imergir cada $b_{\lambda}$ em uma cópia de $\mathbb{Q}$, denotando-a por $B_{\lambda}=\left\{\frac{m b_{\lambda}}{n}: m, n \in \mathbb{Z}, n \neq 0\right\}$. Vejamos que os $B_{\lambda}$ geram uma soma direta. Seja $\mu \in \Lambda$ e suponha $\lambda_{1}, \ldots, \lambda_{k} \in \Lambda, m, m_{1}, \ldots, m_{k} \in \mathbb{Z}$, e $n, n_{1}, \ldots, n_{k} \in \mathbb{N}$ tais que $\frac{m_{1} b_{\lambda_{1}}}{n_{1}}+\cdots+\frac{m_{k} b_{\lambda_{k}}}{n_{k}}=\frac{m b_{\mu}}{n}$. Seja $r=n \cdot n_{1} \cdot \ldots \cdot n_{k}$ e defina $r^{\prime}=\frac{r}{n}$ e $r_{i}=\frac{r}{n_{i}}$. Temos então que $r^{\prime} m b_{\mu}=r \frac{m b_{\mu}}{n}=r\left(\frac{m_{1} b_{\lambda_{1}}}{n_{1}}+\cdots+\right.$ $\left.\frac{m_{k} b_{\lambda_{k}}}{n_{k}}\right)=r_{1} m_{1} b_{\lambda_{1}}+\cdots+r_{k} m_{k} b_{\lambda_{k}}$, o que viola a independência de $\left\{b_{\lambda}: \lambda \in \Lambda\right\}$. Assim, temos que $B:=\bigoplus_{\lambda \in \Lambda} B_{\lambda}<F$, e $B$ é divisível, $\operatorname{logo} F=B \oplus S$; porém como $B$ contém um conjunto independente maximal, devemos ter que $S=\{e\}$, e assim $F=B$, como desejado.

Por fim, temos:

Teorema A.18. Seja $(H,+)$ um grupo divisivel e seja $A \subset H$ enumerável. Então existe $D<H$ divisivel enumerável tal que $A \subset D$.

Demonstração. Pelo Teorema A.17, assumimos que $H=\bigoplus_{\alpha \in I} K_{\alpha}$, em que cada $K_{\alpha}$ é isomorfo a $\mathbb{Q}$ ou a um quasicíclico. Considere $J=\left\{\alpha \in I\right.$ : existe $x \in A$ tal que $\left.x_{\alpha} \neq e\right\}$. Como $A$ é enumerável, $J$ é uma união enumerável de conjuntos finitos, logo enumerável. Defina $D=\{x \in H$ : para todo $\left.\alpha \in I \backslash J, x_{\alpha}=e\right\}$. Então $D \cong \bigoplus_{\alpha \in J} K_{\alpha}$, logo é divisível, enumerável, e $A \subset D$. 


\section{Referências Bibliográficas}

[1] Neil Hindman, Dona Strauss, Algebra in the Stone-Čech compactification: theory and applications (De Gruyter expositions in mathematics: 27) - Walter de Gruyter; Berlin; New York (1998) (1st ed.)

[2] Neil Hindman, Dona Strauss, Algebra in the Stone-Čech compactification: theory and applications - Walter de Gruyter; Berlin; New York (2012) (2nd ed.)

[3] Neil Hindman, Recent results on the algebraic structure of $\beta S$, in "Papers on General Topology and Applications", S. Andima et. al. eds., Annals of the New York Academy of Sciences $\mathbf{7 6 7}$ (1995), 73-84

[4] Neil Hindman, The ideal structure of the space of $\kappa$-uniform ultrafilters on a discrete semigroup, Rocky Mountain Journal of Mathematics, 16 (1985) 685-701

[5] Vitaly Bergelson, Neil Hindman, Nonmetrizable topological dynamics and Ramsey Theory, Transactions of the American Mathematical Society, 320 (1990), 293-320

[6] Neil Hindman, Sums equal to products in $\beta \mathbb{N}$, Semigroup Forum 21 (1980), 221-255

[7] Dona Strauss, $\mathbb{N}^{*}$ does not contain an algebraic and topological copy of $\beta \mathbb{N}$, Journal of the London Mathematical Society, 46 (1992), 463-470

[8] Dona Strauss, Semigroup Structures in $\beta \mathbb{N}$, Semigroup Forum, 44 (1992), 238-244

[9] Neil Hindman, Dona Strauss, The scarcity of products in $\beta S \backslash S$, Topology and Its Applications, 220 (2017), 50-64

[10] László Fuchs, Abelian Groups, Pergamon Press, New York-London-Paris, 1960

[11] Neil Hindman, Dona Strauss, Discrete Groups in $\beta \mathbb{N}$, Topology and Its Applications, 154 (2007), 2099-2103

[12] Yevhen Zelenyuk, Grupos topológicos com semigrupos finitos de ultrafiltros (Russo), Matematychni Studii 6 (1996), 41-52 
[13] Neil Hindman, Dona Strauss, Chains of idempotents in $\beta N$, Proceedings of the American Mathematical Society, 123 (1995), 3881-3888

[14] Dona Strauss, Ideals and commutativity in $\beta \mathbb{N}$, Topology and its Applications 60 (1994), 281293 